Max-Planck-Institut für Biochemie

Abteilung Strukturforschung

Biologische NMR-Arbeitsgruppe

\title{
Structural basis for the regulation of insulin-like growth factors (IGFs) by IGF binding proteins (IGFBPs)
}

Igor Siwanowicz

Vollständiger Abdruck der von der Fakultät für Chemie der Technischen Universität München zur Erlangung des akademischen Grades eines

\section{Doktors der Naturwissenschaften}

genehmigten Dissertation.

Vorsitzender: Univ.-Prof. Dr. J. Buchner

Prüfer der Dissertation: 1. apl. Prof. Dr. Dr. h. c. R. Huber

2. Univ.-Prof. Dr. Dr. A. Bacher

Die Dissertation wurde am 14.02.2005 bei der Technischen Universität München eingereicht und durch die Fakultät für Chemie am 11.05.2005 angenommen. 


\section{Acknowledgements}

I would like to thank everyone who contributed to this work.

In particular I am grateful to Professor Robert Huber for giving me the opportunity to work in his department and for being my Doktorvater.

To my supervisor Doctor Tad A. Holak, for his support, discussions and encouragement.

To the laboratory team: Dorota Ksiazek, Grzegorz Popowicz, Joma Joy, Loy D'Silva, Madhu Ghosh, Magda Wisniewska, Mahavir Singh, Marcin Krajewski, Mariusz Kamionka, Ola Mikolajka, Pawel Smialowski, Przemyslaw Ozdowy, Sudipta Majumdar, Till Rehm Tomasz Sitar and Ulli Rothweiler, for creating one of the kind, motivating atmosphere of competence and creativity.

My special thanks go to Joma, Mahavir, and Sudipta - for the supply of Bollywood blockbusters and the discussion of thereof; Christine Breitenlechner - for help in the matters requiring the knowledge of German language and bureaucracy; Narashima and C. Vinula - for being such unusual specimens; Grzegorz, the source of Anime artworks and creations, for the ideas and discussions usually not related to the subject of my studies; Magda - for her patience and care. 


\section{Publications}

Parts of this thesis have been or will be published in due course:

Siwanowicz, I., Popowicz, G.M., Wisniewska, M., Huber, R., Kuenkele, K-P., Lang, K., Engh, R.A., and Holak, T.A. (2005) Structural basis for the regulation of insulin-like growth factors by IGF binding proteins. Structure 13 (in press).

Siwanowicz, I., Popowicz, G.M., Ghosh, M., Moroder, L., Dsilva, L., Joy, J., Majumdar, S., Wisniewska, M., Firth, S.M., Baxter, R.C., Huber, R., and Holak T.A. (2005) Molecular architecture of the insulin-like growth factor binding proteins (IGFBPs). J. Biol. Chem. (submitted).

Ghosh, M., Shanker, S., Siwanowicz, I., Mann, K., Machleidt, W., and Holak, T.A. (2005) Proteolysis of insulin-like growth factor binding proteins (IGFBPs) by calpain. Biological Chemistry 386 (in press).

Budisa, N., Pipitone, O., Siwanowicz, I., Rubini, M., Pal, P.P., Holak, T.A. and Gelmi, M.L. (2004) Efforts towards the design of 'Teflon' proteins: In vivo translation with trifluorinated leucine and methionine analogues. Chemistry \& Biodiversity 1, 14651475. 


\section{Contents}

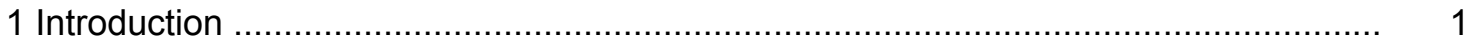

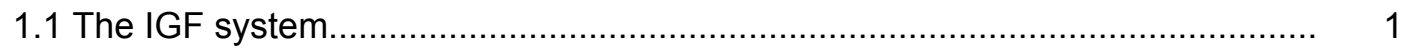

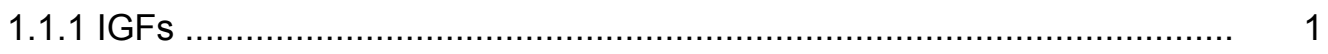

1.1.2 IGF receptors ................................................................... 4

1.1.2.1 IGF receptor signaling ..................................................... 6

1.1.3 Insulin-like growth factor binding proteins ....................................... 9

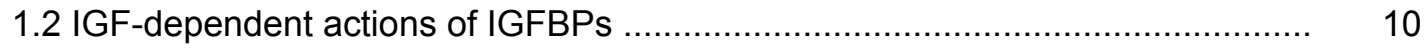

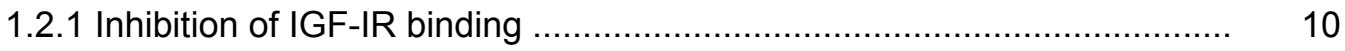

1.2.2 Modulation of IGF binding activity of IGFBPs ................................... 11

1.2.2.1 Association of the IGFBPs to the extracellular matrix (ECM) or specific cell membranes .................................................... 11

1.2.2.2 Cleavage of the IGFBPs by specific proteases ......................... 12

1.2.2.3 Dephosphorylation ............................................................. 13

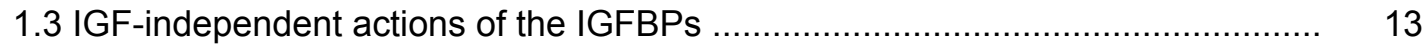

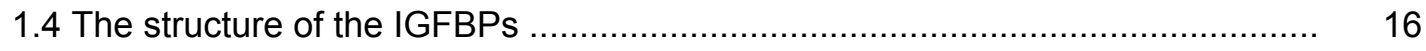

1.4.1 Posttranslational modifications ...................................................... 18

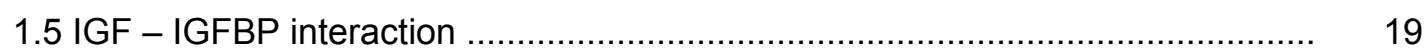

1.5.1 IGF residues involved in IGFBP binding ...................................... 19

1.5.2 IGFBP residues involved in IGF binding ....................................... 20

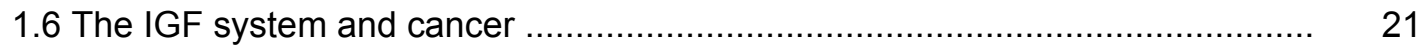

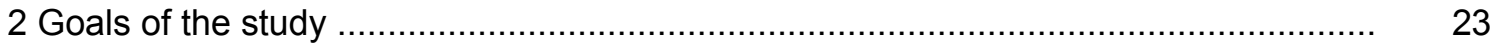

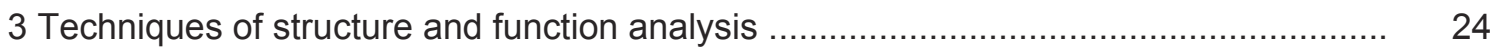

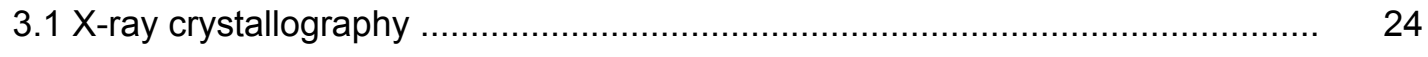

3.1.1 Crystal space groups and symmetries .......................................... 24

3.1 .2 Crystallization ..................................................................... 24

3.1.3 X-ray diffraction .................................................................... 26

3.1.4 Solving the "phase problem" ....................................................... 26

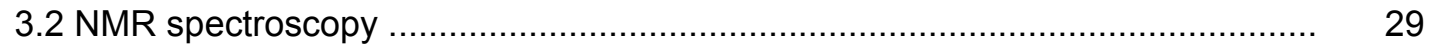

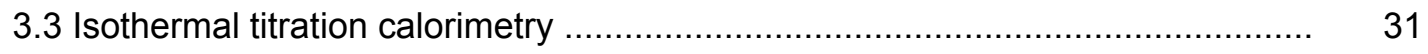

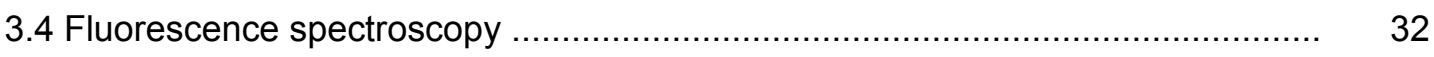

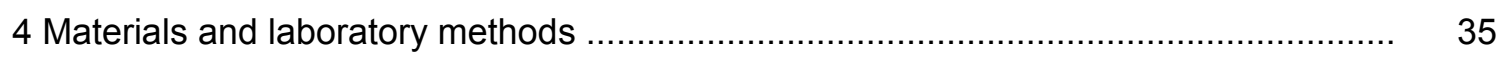

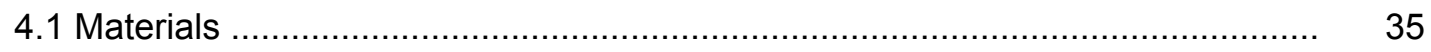


4.1.1 E. coli strains and plasmids ...................................................... 35

4.1.2 Cell growth media and stocks .......................................................... $\quad 35$

4.1.3 Protein purification - buffers ...................................................... 38

4.1.4 Reagents and buffers for the SDS-PAGE ....................................... 40

4.1.5 Enzymes and other proteins ....................................................... 42

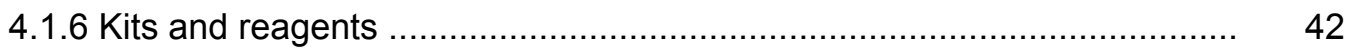

4.1.7 Protein and nucleic acids markers ....................................................... 43

4.1.8 Chromatography equipment, columns and media ............................. 43

4.2 Laboratory methods and principles ........................................................ 43

4.2.1 Constructs' design .............................................................................. 43

4.2.2 Choice of the expression vector ..................................................... 44

4.2.3 DNA techniques ...................................................................... 46

4.2.3.1 Preparation of plasmid DNA ............................................. 46

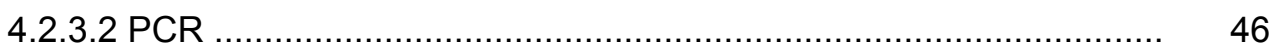

4.2.3.3 Digestion with restriction enzymes ......................................... 48

4.2.3.4 Purification of PCR and restriction digestion products ................. 48

4.2.3.5 Mutagenesis of the miniNBP-5 and CBP-4 ….......................... 49

4.2.3.6 Agarose gel electrophoresis of DNA …................................... 50

4.2.4 Transformation of $E$. coli ............................................................. 50

4.2.4.1 Transformation of chemically competent cells ............................ 51

4.2.4.2 Transformation by electroporation ..................................... 51

4.2.5 Protein chemistry methods \& techniques ...................................... 51

4.2.5.1 Sonication .......................................................................... 51

4.2.5.2 SDS polyacrylamide gel electrophoresis (SDS PAGE) ............... 52

4.2.5.3 Visualization of separated proteins ............................................ 52

4.2.5.4 Protein expression, refolding and purification ........................... 52

4.2.5.5 Determination of protein concentration .................................... 54

4.2 .6 X-ray crystallography ............................................................... 54

4.2.6.1 protein crystallization ..................................................... 54

4.2.6.2 Data collection and molecular replacement ............................... 56

4.2.7 NMR - experimental procedure ....................................................... 59

4.2.8 Isothermal titration calorimetry ….................................................... 59

4.2.9 Fluorescence measurements .................................................... 60

4.2.10 IGF-IR autophosphorylation inhibition assay ................................. 60

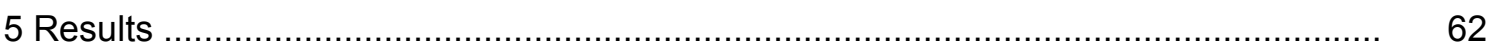

5.1 Cloning, expression and purification of IGFBP domains ................................. 62 
5.1.1 Protein purification strategies ................................................... 62

5.1.2 Solubilization of inclusion bodies ...................................................... 66

5.1.3 Immobilized metal affinity chromatography (IMAC) ............................... 66

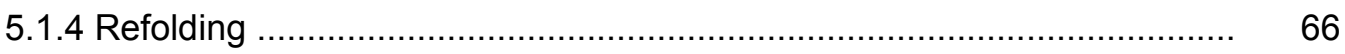

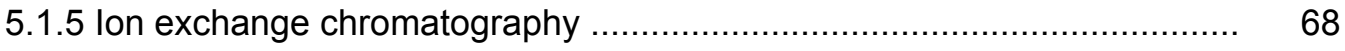

$5.1 .6 \mathrm{Gel}$ filtration chromatography .................................................. 68

5.2 Preliminary functional and structural studies ................................................ 68

5.2.1 Gel filtration mobility shift assay .......................................................... 68

5.2.2 NMR studies on the folding and domain organization of IGFBPs ........... 71

5.2.3 Optimization of the carboxyl-terminal construct of IGFBP-4 .................. 71

5.3 Structure of IGFBP-4 …................................................................... 74

5.3.1 Crystallization of the binary and ternary complexes ........................... 74

5.3.2 Overall structure of the NBP-4(3-82)/IGF-I binary complex .................. 75

5.3.3 NBP4(3-82)/IGF-I vs. NBP4(1-92)/IGF-I ...................................... 80

5.3.4 The subdomain organization of NBP-4 …...................................... 82

5.3.5 Ternary complex NBP-4(3-82)/IGF-I/CBP-4(151-232) ........................... 83

5.3.6 The IGF-I/NBP-4 interaction - implications for IGF-I binding

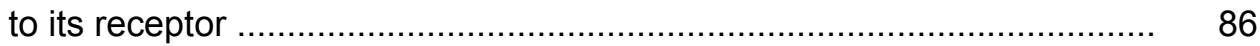

5.4 Functional studies of IGFBP-4 - IGF interaction ........................................ 86

5.4.1 Binding among domains of IGFBP-4 and IGF as studied with NMR and ITC measurements ........................................................... 86

5.4.2 Inhibition of the IGF-I receptor binding and action by IGFBP fragments .. $\quad 90$

5.4.3 Fine-tuning of the N-terminal "thumb" residues .................................... 91

5.4.4 Characterization of the thumb microenvironment in the IGFBP/IGF-I complex by fluorescence spectroscopy .............................................. 91

5.5 Isoquinoline compound NBI-31772 does not cause dissociation

of the IGF-I/IGFBP complex ............................................................. 94

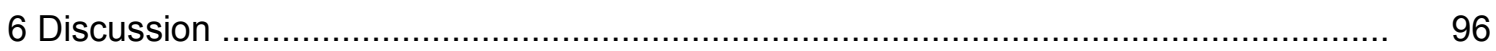

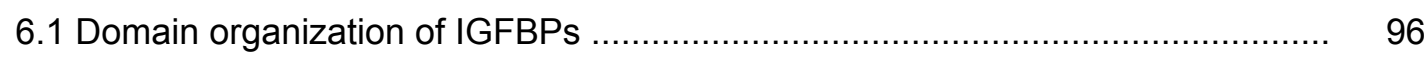

6.2 Contributions of the $\mathrm{N}$ - and C-terminal domains to IGF-I binding …................. 96

6.3 The IGF-I/NBP-4/CBP-4 interaction .................................................... 98

6.4 Inhibition of the IGF-I receptor binding and action by IGFBP fragments ............. 103

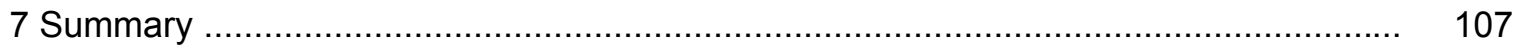

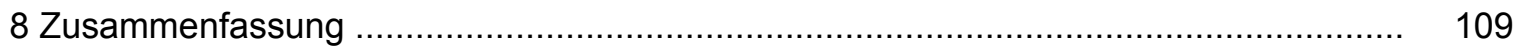

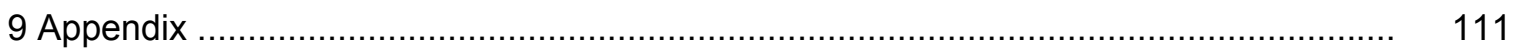

9.1 Abbreviations and symbols ............................................................ 111 
9.2 Isothermal titration calorimetry

9.3 IGF-IR autophosphorylation inhibition assay

10 References ..... 


\section{Introduction}

\subsection{The IGF system}

The identity and function of a developing cell is predestined by the stimuli it receives from the surrounding environment. Contacts with neighboring cells and signals received from membrane receptors, which are activated by various specific growth factors, modulate a gene expression pattern of the cell, leading to differentiation. The very same stimuli decide fates of the mature cell, i.e. the rate of protein and nucleic acid synthesis, cell cycle progression, and survival depend on the presence of cell-tocell junctions and constant supply of the protein factors. In a cell deprived of this steady stimulation, anabolic pathways cease and suicidal pathways take over, leading to apoptosis. Deregulation of extracellular developmental signals may lead to an opposite extreme situation: quiescent cells commence excessive proliferation, the first step in cancerogenesis.

It is an ensemble of mutually interacting proteins referred to as "the IGF system" that plays the key role in regulation of cell development, metabolism and survival through its life. The IGF system involves two ligands, insulin-like growth factor-I and -II (IGF-I and IGF-II) that interact with specific type 1 or type 2 IGF receptors (IGF-IR and IGF-IIR). There are six high affinity IGF binding proteins (IGFBPs) modulating IGF availability and bioactivity.

\subsubsection{IGFs}

Salmon and Daughaday first identified insulin-like growth factors as "sulphation factor activity" (Daughaday et al., 1972). Later the term "somatomedin" was introduced, which was replaced by "insulin-like growth factor" because of its structural homology with insulin (Rinderknecht and Humbel, 1976). Rinderknecht and Humbel revealed the amino acid sequences of both IGF-I and IGF-II in 1978 (Rinderknecht and Humbel, 1978a; 1978b). Almost all cell types express IGF receptors, and therefore respond to IGF stimulation of growth and differentiation. Both IGF-I and -II are required for normal embryonic and fetal development. Postnatally IGF-I mediates the growth-promoting effect of growth hormone $(\mathrm{GH})$ (Schlechter et al., 1986; Daughaday et al., 1999) and is 
the major regulator of animal skeletal growth, maintaining bone mass throughout life. IGF-I augments wound healing by acting at several sites during the repair process (Chan and Spencer, 1998; Suh et al., 1992). Also, nerve cell survival and regeneration is promoted by IGF-I (Leventhal et al., 1999; Jung et al., 1998). IGF-I is anti-apoptotic and autocrine production by tumors is common. In the kidney, IGF-I increases the glomerular filtration rate, phosphate transport, and the activity of 25-hydroxyvitamin D1- $\alpha$-hydroxylase (Halloran and Spencer, 1988). The primary regulator of IGF-I expression is growth hormone. Developmental expression of IGF-I in various tissues precedes however that of $\mathrm{GH}$, supporting an independent role of IGF-I in embryonic and fetal life.

Human IGF-I and IGF-II are single-chain polypeptides. IGF-I is a basic peptide and contains 70 amino acids, with IGF-II being slightly an acidic protein containing 67 amino acids. The amino acids of both IGF-I and -II are grouped into domains A and B (similar to insulin), domain $\mathrm{C}$ (analogous to the connecting peptide of proinsulin) and the C-terminal octapeptide (D region, 63-70) that has no counterpart in insulins and proinsulins (Baxter et al., 1992). Both IGF factors contain three disulphide bonds, and display approximately 62 percent sequence homology with each other and 47 percent with insulin (Figure 2). In addition to the classical IGF-I molecules a truncated form of IGF-I known as DES(1-3)IGF-I has been found in fetal and adult human brain (Carlsson-Skwirut et al., 1986; Sara et al., 1986; Humbel, 1990). DES(1-3)IGF-I is the product of differential processing of pro-IGF-I lacking the first three residues at the amino terminus: Gly-Pro-Glu (GPE). The biological potency of this truncated form is 10 times higher than that of the full-length form and is explained by reduced binding to IGF-binding proteins (Francis et al., 1988; Beck et al., 1993; Carlsson-Skwirut et al., 1989; Ballard et al., 1996).

The 3-dimensional crystal structure of insulin has been first determined in the late 1960s (Adams et al., 1969), and several structures of its variants, mutants and oligomeric forms of insulin are known today. This is in contrast to IGFs, for which no high-definition structure existed until recently. The structures of IGFs obtained from NMR studies were of low resolution due to the tendency of the proteins to aggregate at higher concentrations and $\mathrm{pH}$ values $>3$. These studies confirmed however the consistency of the solution structure of IGF-I with its model, deduced from the porcine insulin structure (Blundell et al., 1978; Cooke et al., 1991; Sato et al., 1993). Only very recently two crystal structures of IGF-I have been published: in a complex with amino- 


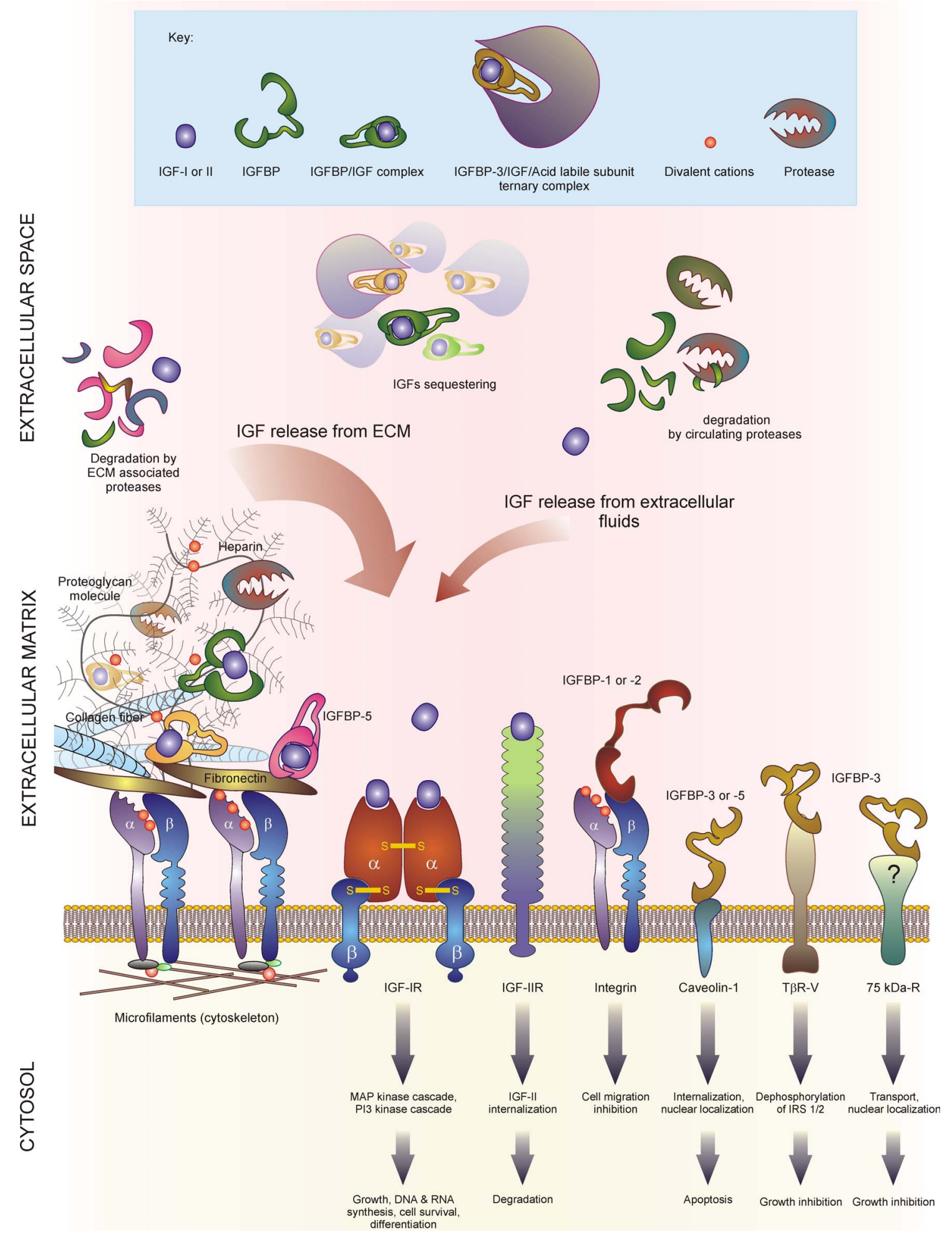

Figure 1. IGF-dependent and independent actions of IGFBPs. 1) IGFBPs sequester IGFs by forming inactive complexes, the predominant form being IGFBP-3/IGF-I/ALS ternary complex. 2) IGFs are freed from these complexes when proteases degrade IGFBPs or 3) when IGFBPs interact with cell surfaces or ECM. 4) Binding of IGFBP-1 or -2 to integrins, and IGFBP-3 or -5 toT $\beta R-V$, caveolin or unknown protein receptors, modulates their internalization and IGFindependent actions. 
terminal fragment of IGFBP-5 (miniNBP-5, residues 40-92) (Zeslawski et al., 2001) and with a bound CHAPS detergent molecule (Vajdos et al., 2001). IGF-I is composed primarily of three helical segments corresponding to the B-helix (IGF-I residues 7-18) and two A-helices (IGF-I residues 43-47 and 54-58) of insulin. The hydrophobic core includes three disulfide linkages between Cys6 and Cys48, Cys18 and Cys61, and Cys47 and Cys52. Residues of the C-chain (Ser34-Thr41) form a flexible loop, and no electron density could be observed for the region in both crystallographic studies quoted above. Similarly, two N-terminal and six C-terminal residues showed no electron density, indicating motional flexibility in these regions. The study presented in this thesis provides the first high-resolution view of the conformation of these biologically important regions.

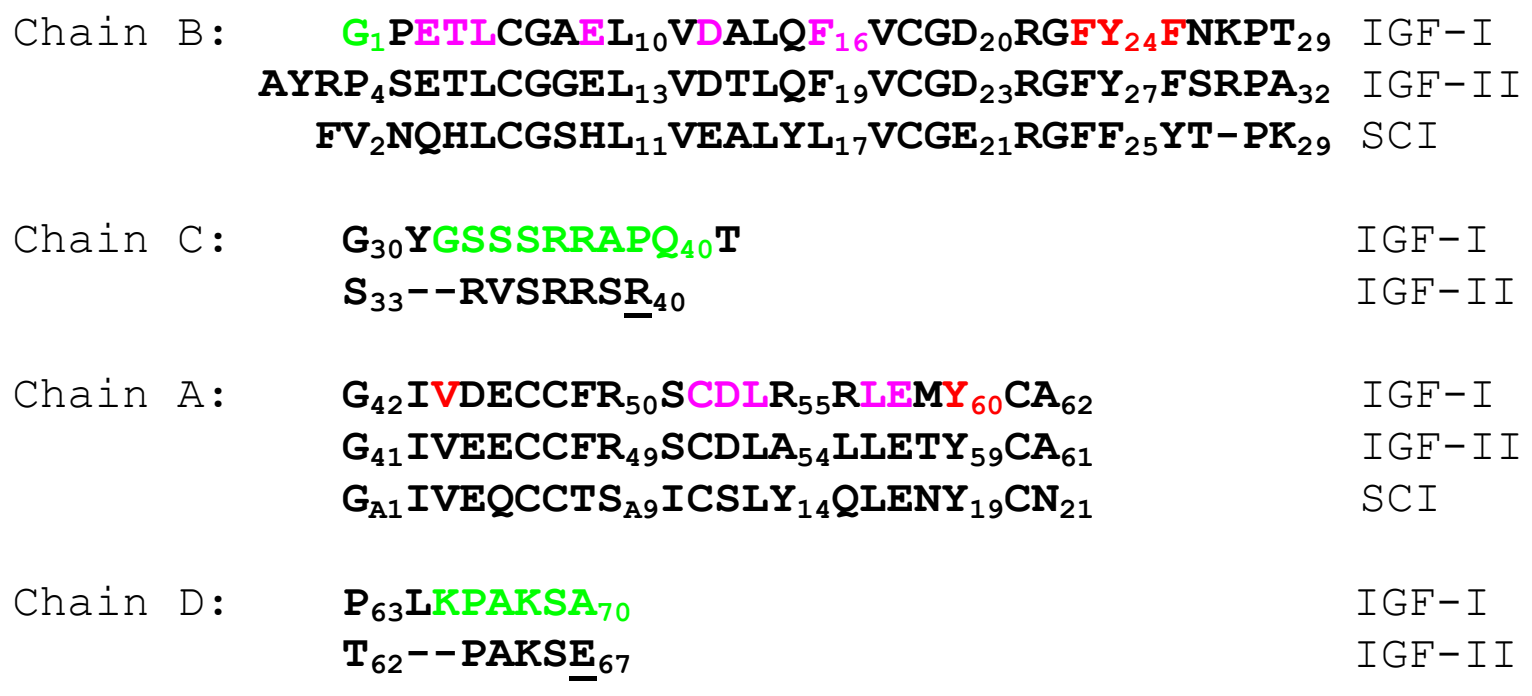

Figure 2. Sequence and structure alignment of IGFs and single-chain insulin (SCI). Residues that make contacts with miniNBP- 5 within $4 \AA$ are highlighted in magenta; residues responsible for binding to IGF-IR in red, residues in green showed no electron density in the structure of the miniNBP-5/IGF-I binary complex.

\subsubsection{IGF receptors}

The IGFs interact with specific cell surface receptors, designated type I and type II IGF receptors (IGF-IR and IGF-IIR). The growth promoting, differentiation and antiapoptotic effects of IGFs result from interaction of free IGF with IGF-IR, a transmembrane, heterotetrameric type II tyrosine kinase that is homologous to the insulin receptor (reviews: De Meyts et al., 1994; LeRoith et al., 1995, LeRoith, 2000). The IGF-IR is a membrane glycoprotein of $300-350 \mathrm{kDa}$, consisting of two $\alpha$-subunits 
(135 kDa each) and two $\beta$-subunits (90 kDa each) (Figure 1) (Rechler, 1985; Yamasaki et al., 1993). Disulphide bonds connect both alpha and beta-subunits to form a functional heterotetrameric receptor complex. In analogy with the insulin receptor, IGFI receptor subunits are encoded within a single $180 \mathrm{kDa}$ polypeptide precursor that is glycosylated, dimerised and proteolytically processed to yield the mature alpha-2-beta2 -form of the receptor. The $\alpha$-subunit is entirely extracellular and contains the ligandbinding site, a cysteine-rich domain. The $\beta$-subunit contains the hydrophobic transmembrane domain with a short extracellular region, and a tyrosine kinase domain in its cytoplasmic portion. However, the mature brain also expresses a structural subtype of the IGF-I receptor, which appears to be unique to differentiated neurons. This receptor consists of a lower mass $\alpha$-subunit of 115 kDa (Heidenreich et al., 1986; Burgess et al., 1987). The structure of the first three domains of the extracellular portion of IGF-IR (L1-Cys-rich-L2, residues 1-462) has been determined to a $2.6 \AA$ resolution (Garrett et al., 1998). The $L$ domains ( $L$ stands for "large") each adopt a compact shape consisting of a single-stranded right-handed $\beta$-helix. The Cys-rich region is composed of eight disulphide-bonded modules, seven of which form a rodshaped domain. The receptor fragment is not active in terms of IGF binding.

Information on ligand binding has come from chemical crosslinking with derivatised insulin or IGFs and from the analysis of receptor chimaeras and receptors with point mutations (reviewed in: Adams et al., 2000). The residues of IGF-I and -II engaged in binding of IGF-IR have been mapped in a number of studies (Cascieri et al., 1988 and 1989; Bayne et al., 1990; Sakano et al., 1991; Roth et al., 1991; Darren et al., 1996). The topic of IGF/IGF-IR interaction is covered in detail in the Discussion section of this thesis.

The physiological functions of insulin and IGFs are very different, while their receptor structures are similar. Tissue distribution of the individual receptors, the specificity of the ligand-receptor interaction, the rate of internalization and the small structural differences between the two receptors explain the well-characterized divergent physiological functions of insulin and IGFs. For example, the liver expresses only the insulin receptor and fat cells express mainly IRs. For this reasons these tissues display metabolic responses such as glucose uptake and lipid synthesis. Further, the IGF-IR ligands include IGFs and insulin. The IGF-IR binds IGF-I with a high affinity (ca. $0.1 \mathrm{nM}$ ), IGF-II with a 10-fold lower affinity, and insulin with a 1001000 fold lower affinity (Bohannon et al., 1988; Lesniak et al., 1988). On the other 
hand, IR demonstrates high-affinity binding to insulin (ca. $0.1 \mathrm{nM}$ ), 10-fold lower affinity binding to IGF-II, and a 100-fold lower affinity binding to IGF-I. The IR is mainly involved in short-term metabolic effects, while the IGF-IR is predominantly involved in long-term growth/mitogenic effects (Rechler, 1985; LeRoith et al. 1995). The structural differences between both receptors are also involved in different intracellular signaling. For example, the IR contains two distal tyrosine residues, whereas the IGF-IR contains three tyrosine residues, and the tyrosine kinases of both receptors show distinct preferences for the tyrosine phosphorylation domains of insulin receptor substrate 1 (IRS 1) (De Meyts et al., 1994).

The IGF-II receptor (IGF-IIR) consists of a single glycosylated polypeptide of approximately $260 \mathrm{kDa}$. It is primarily extracellular, having a short cytoplasmic tail and consists of 15 repeat mannose-6-phosphate (M6P)-binding units (August et al., 1983; Kornfeld, 1992). In 1987, Morgan and coworkers cloned the IGF-II receptor and revealed that it is identical to the cation-independent mannose-6-phosphate (Man-6-P) receptor that is involved in transport of Man-6-P-bearing lysosomal enzymes from their sites of synthesis into an endosomal/pre-lysosomal compartment (Morgan et al, 1987; reviews: Braulke, 1999, Hassan, 2003). This finding was confirmed by showing that the IGF-II receptor binds independently both IGF-II and lysosomal enzymes that contain M6P. Several studies have shown that the binding sites for IGF-II and M6P on the receptor are distinct, and that the receptor can bind both ligands simultaneously (MacDonald et al., 1988; Nissley and Kiess, 1991).

IGF-IIR binds IGF-II with high affinity but interacts minimally with IGF-I and does not bind to insulin at all. Gene targeting studies revealed that IGF-IIR is important for the control of embryonic growth, and for internalization and degradation of extracellular IGF-II (reviews: Braulke, 1999; Hassan, 2003), however, it is unclear whether this receptor is involved in IGF-II signaling. The M6P/IGF-IIR was identified as a putative "IQ gene". Comparing Caucasian children with an IQ of 160 or higher with those with an average $I Q$, showed that the M6P/IGF2R is linked to human cognitive ability (Chorney et al. 1998).

\subsubsection{IGF receptor signaling}

IGF-I binding to a cysteine-rich domain in the extracellular $\alpha$ subunit of IGF-IR causes autophosphorylation of three tyrosines in the activation loop of the tyrosine kinase domain in the cytoplasmic portion of the $\beta$ subunit, which results in amplification of 
tyrosine kinase activity and further autophosphorylation of additional tyrosine residues. These phosphotyrosine containing motifs are binding sites for adaptor and effector molecules in receptor signaling pathways, including insulin receptor substrates and Src homology/collagen (Shc), which are subsequently phosphorylated on their tyrosines (reviewed in White and Kahn, 1998; Kim et al., 1998). The IRSs are known as "docking" proteins and constitute a family of four structurally related adaptor proteins that can link the IGF-I receptor to downstream signal transduction mediators regulating cellular growth. IRS 1 is the most extensively studied, and has multiple tyrosines, which associates with $\mathrm{SH} 2$ domain-containing proteins including growth factor receptor bound-2 protein (Grb2) and p85 regulatory subunit of phosphoinositol-3 (PI-3) kinase, p110. phosphorylation of IRS 1 and 2 leads to activation of two downstream signaling cascades: 1) the mitogen-activated protein kinase (MAPK) and the 2) phosphatidylinositol 3-kinase (P3K) cascades (Figure 3).

1) Stimulation of growth factor receptor-bound protein 2 (Grb2) by activated IRS $1 / 2$ or Shc leads to the coupling of Grb2 with the guanyl nucleotide exchange factor son of sevenless (Sos), which in turn activates ras. In the ras pathway, phosphorylation of c-raf 1 activates the downstream protein kinase, MAP kinase kinase 1 (also known as MEK or MKK1) or MAP kinase kinase 2 (MKK2). MKK1 and MKK2 activate two members of the MAP kinase family; extracellular signal related protein kinase (ERK)-1 and -2. Phosphorylated ERK-1 and (or) ERK-2 are translocated to the nucleus where in turn they phosphorylate a variety of transcription factors including cfos, c-jun and c-myc. This signaling cascade is important for cellular growth and mitogenesis (Hernandez-Sanchez et al., 1995). The c-Myc protooncogene encodes a nuclear transcription factor that is both necessary and sufficient to trigger entry of certain cell types into the S-phase of the cell cycle (Cole, 1986).

2) IGF-IR-activated IRS 1 binds the regulatory p85 alpha-subunit of PI-3 kinase, and this complex activates the $110-\mathrm{kDa}$ catalytic subunit, which phosphorylates phosphoinositides, generating $\mathrm{Pl}-3$ phosphate, $\mathrm{PI}-3$, 4-bisphosphate, and $\mathrm{PI}-3,4,5-$ triphosphate (reviewed in Myers and White, 1995). These phosphatidylinositides in turn activate protein kinases including p70 ribosomal protein $S 6$ kinase $\left(p 70^{\mathrm{s} 6 \mathrm{k}}\right)$, and protein kinase B (PKB/Akt) as well as other less characterised downstream molecules (Datta et al., 1997; Du and Montminy, 1998; Myers et al., 1994). This pathway is important for IGF-mediated survival, enhancement of cellular motility and protein synthesis, retaining of cell-to-cell junctions and protection from programmed cell death. 


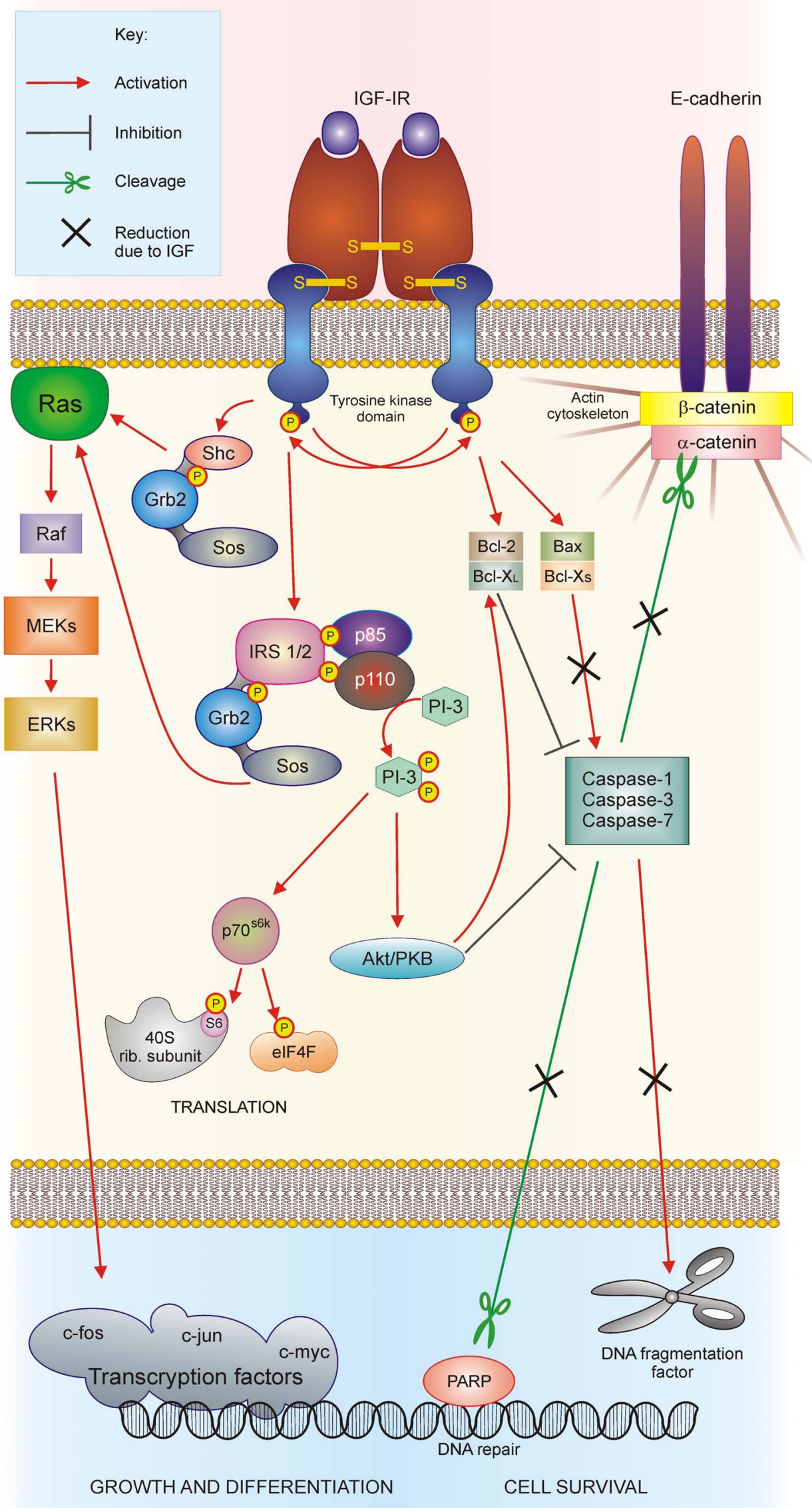


Figure 3. Schematic presentation of the intracellular signaling pathways of the IGF-I receptor. The initial response of the IGF-IR to IGF-I binding is to undergo autophosphorylation on specific tyrosine residues. The intrinsic tyrosine kinase activity of the IGF-I receptor is enhanced and phosphorylates multiple substrates, including IRS 1 and IRS 2, on tyrosine residues. Proteins containing SH2-domains such as PI-3 kinase (p85/p110) and Grb2, associate with specific phosphotyrosine-containing motifs within IRS 1 and IRS 2. A similar response occurs with Shc. The ras/raf/MAP kinase pathway is activated by the association of IRS 1 and Shc with Grb2, which in turn is associated with the mammalian guanine nucleotide exchange factor protein son of sevenless (Sos). The PI-3 kinase/Akt cascade plays primary role in regulation of apoptosis. Activated Akt kinase phosphorylates proapoptotic factor Bad on a serine residue, resulting in its dissociation from anti-apoptotic protein, B-cell lymphoma $-X$ $\left(\mathrm{Bcl}-\mathrm{x}_{\mathrm{L}}\right)$. The anti-apoptotic protein $\mathrm{Bcl}-2$ and apoptotic proteins $\mathrm{Bax}$ and $\mathrm{Bcl}-\mathrm{x}_{\mathrm{s}}$ are also phosphorylated either by Akt or IGF-IR. This and Akt pathway converge on the inhibition of caspases, especially caspase-3, which is then blocked from performing an apoptosis-initiating cleavage of poly(adenosine diphosphate ribose) polymerase (PARP) and blocked from degrading $\beta$-catenin, part of the cadherin cell-adhesion system. Akt activates $p 70^{\text {s6k }}$, which in turn phosphorylates S6 protein of 40 S ribosomal subunit and translation initiation factor-4 (elF4F), resulting in a stimulation of protein synthesis

\subsubsection{Insulin-like growth factor binding proteins}

IGFs are unlike other endocrine hormones, such as growth hormone and insulin, which are secreted from storage vesicles in an endocrine gland in response to releasing factors such as $\mathrm{GH}$ releasing factor or glucose. In contrast, the IGFs are not stored in an endocrine gland and are therefore not released acutely into the blood by releasing factors. However, the IGFs are stored in the blood and in tissues in a different, and somewhat unique manner for a protein hormone; they are stored bound to specific IGFBPs.

IGFBPs are a class of six multifunctional, homologous proteins. The precursor forms of all six IGFBPs have secretory signal peptides of between 20 and 39 amino acids, and the mature proteins are all found extracellularly. All IGFBPs bind IGFs with high affinity but in addition possess several specific, distinct functional binding sites that are not shared. The latter confer unique biological properties to each IGFBP. The common major binding reaction is the association with IGFs that regulates their free level and hence all the important cellular activities of the IGFs discussed above. Most of the IGF-I and IGF-II molecules in serum are found in a 150-kDa ternary complex formed by an IGF, IGFBP-3 - the predominant IGFBP in plasma, and a $85 \mathrm{kDa}$ leucine-rich glycoprotein known as the acid labile subunit (ALS) (Hashimoto et al, 1997; Boisclair et al., 2001; Baxter, 1994). To a smaller degree, IGFBP-5 also forms such a complex (Twigg et al., 2000). The ALS-protein plays an important role in regulating the affinity of IGFBP-3 to IGF-I, thus regulating the levels of free IGFs. In 
addition, ALS-proteins increase the molecular mass of the IGF-IGFBP-3 complex so that the access of the circulating IGFs to the extracellular fluid and thus to the various tissues is limited. IGFs are released from the 150-kDa complexes by limited proteolysis of IGFBP-3 and cross the vascular endothelial barrier alone or associated with smaller IGFBPs.

The other $20-25 \%$ of the IGFs is associated with lower mass IGFBPs, and less then $1 \%$ of IGFs circulate in the free forms (Baxter, 1994; Hashimoto et al, 1997). However, not only can IGFBPs inhibit IGF actions, all, except IGFBP-4, can potentiate specific activities of the IGFs under certain experimental conditions (reviewed in Firth and Baxter, 2002). In addition, the IGFBPs have IGF-independent receptor-mediated actions on cells, which can also be inhibitory or stimulatory (see below).

\subsection{IGF-dependent actions of IGFBPs}

It is broadly agreed that the functions of IGFBPs which regulate the endocrine actions of IGFs are: 1) regulation of the rate of transport of IGFs from the vascular compartment to enhance the growth-potentiating effects of IGFs, 2) prolongation of the plasma half-lives of IGFs and 3) inhibition of the insulin-like activity of IGFs.

\subsubsection{Inhibition of IGF-IR binding}

IGFBPs regulate the bioactivity of IGFs in several ways. They transport the IGFs to and from the extracellular fluid and control the level of the biologically active, free IGFs since binding partially inactivates the IGFs. As a consequence of the high affinities of the IGFBPs only ca. $0.5 \%$ of IGFs in the plasma are free to act and thus IGFs do not produce hypoglycemia physiologically because of the IGFBPs (Martin and Baxter, 1999). Since their affinity is 10-fold higher, the IGFBPs compete favorably with the tissue IGF receptors and can inhibit the stimulatory actions of IGFs on cellular proliferation, migration and differentiation. IGFBPs 1, 2, 3 and 5 have been shown to block the anti-apoptotic effects of IGFs on a variety of normal and malignant cells (Martin and Baxter, 1999). IGFBPs also prolong the half-life of IGFs, increasing it from 7-9 $\mathrm{min}$ to $30-40 \mathrm{~min}$. In plasma the half-life is increased to 18 hours because once IGFs bind IGFBP-3, the complex associates with the acid labile subunit. 


\subsubsection{Modulation of IGF binding activity of IGFBPs}

The IGFBP interaction with IGF can change from a high affinity stable complex to a highly labile one when IGF release is required. In the tissues the release of IGFs from the IGFBPs can be modulated by three mechanisms, which function to decrease the affinity of the IGFBPs to the IGFs and act as a sustaining local source of IGFs to the IGF receptor.

1.2.2.1 Association of the IGFBPs to the extracellular matrix (ECM) or specific cell membranes

The unique ability of IGFBPs to potentiate rather than inhibit certain actions of the IGFs involves tissue association of the IGFBPs. Cell surface and ECM association is one of the possible mechanisms to alter the affinity of IGFBPs for the IGFs. Cell associated IGFBP-3 and -5 have a 3 to 40 -fold lower affinity for IGF-I than that of free, soluble proteins. For IGFBP-3, a 40-fold lower IGF-I affinity was reported when bound to the human fibroblast cell lines GM10 and T98G compared with IGFBP-3 in solution (McCusker et al., 1997); IGFBP-2, which associates with cell-surface proteoglycans in the brain, shows a threefold decrease in IGF affinity when bound to chondroitin sulfate (Russo et al., 1997). The partition between membrane associated and soluble IGFBPs seems to be multivalent cation dependent. Zinc was shown to prevent the loss of cellassociated IGFBP-3 and -5 , and at the same time lower their IGF binding affinity (McCusker et al., 1990, 1997; Sackett and McCusker, 1998a,b). Simultaneously, zinc increases the affinity of the IGF-IR for both IGF-I and -II (McCusker et al., 1998). Metal ions interact with IGFBP-3 and -5 through a specific metal binding site, localized in the C-domain (Singh et al, 2004).

Components of cell membranes and extracellular matrix that interact with IGFBPs include proteoglycans, heparin (Andress, 1995), integrin (Jones et al., 1993), fibronectin (Xu et al., 2004) and vitronectin (Nam et al., 2002). Only vitronectin has been shown unequivocally to modulate IGF binding by IGFBP-5 directly. Binding of fibronectin to IGFBP-5 had no effect on its ability to bind IGF-I, but it increased the proteolytic degradation of IGFBP-5, and hence IGF release (Xu et al., 2004). Certain proteases, such as thrombin or pregnancy-associated plasma protein-A (PAPP-A) have been shown to be present in the extracellular matrix of human tissues. Presence of the proteolytic enzymes in ECM may account for degradation of IGFBPs directed to this compartment (see below). Both IGFBP-1 and IGFBP-2 have an Arg-Gly-Asp 
(RGD) motif, which was shown to bind $\alpha 5 \beta 1$ integrin and consequently to mediate cell surface association of these IGFBPs (Jones et al., 1993). In this circumstance, integrin-bound IGFBP could act as a reservoir for IGF, which provides a high concentration of IGF to stimulate the IGF-I receptor. There is no evidence for cell surface association of IGFBP-4 (Kelley et al. 1996), suggesting that IGFBP-4 exists primarily in a soluble extracellular form and this may be why IGFBP-4 is generally considered the only purely inhibitory IGFBP.

\subsubsection{Cleavage of the IGFBPs by specific proteases}

Each of the six IGFBPs can undergo proteolysis, which results in a generation of fragments with markedly decreased affinity for IGFs. Members of several classes of proteases have shown the ability to cleave IGFBPs, including kallikreins, cathepsins and matrix metalloproteases. While some of the IGFBP proteases can use multiple IGFBPs as substrate, there are apparently proteases that are specific for individual IGFBPs (reviews: Maile et al., 1999, Schneider et al., 2002). Proteolysis is a major regulatory mechanism of IGFBP-4 functions. An IGF-dependent IGFBP-4-specific protease was first reported in the media conditioned by both human and sheep dermal fibroblasts (Fowlkes and Freemark, 1992). This proteolytic activity has also been detected in the conditioned media from human osteoblasts (Qin et al., 1999), vascular smooth muscle cells (Bayes-Genis et al., 2001), granulosa cells (Conover et al. 2001), trophoblast and decidualized endometrial stromal cells (Giudice et al., 2002), as well as in ovarian follicular fluid (Conover et al., 1999) and human pregnancy serum (Byun et al. 2001b). The protein factor responsible for this activity was identified as pregnancy-associated plasma protein-A (PAPP-A) (Lawrence et al., 1999a). As the name implicates, PPAP-A was first isolated from human pregnancy serum (Lin et al., 1974) and belongs to the large metzincin family of metalloproteases (Lawrence et al. 1999b; Boldt et al., 2001). It cleaves IGFBP-4 at a single site, between Met135/Lys136 in human IGFBP-4 (Byun et al., 2000, Laursen et al. 2002). IGFBP-4 cleavage by PAPP-A uniquely depends on the presence of IGF (Byun et al., 2000; Qin et al., 2000; Laursen et al., 2001). Recent data suggest that IGFs enhance the proteolysis by binding to IGFBP-4, but not by interaction with PAPP-A (Qin et al., 2000; Laursen et al., 2001). PAPP-A can also cleave bovine IGFBP-2 between Gln165/Met166 in an IGF-dependent manner (Monget et al. 2003). 
Number of calcium-dependent serine proteases have been reported to degrade IGFBPs (Nam et al., 1994; Thrailkill et al., 1995; Kanzaki et al., 1994). Thrombin was found to cleave IGFBP-5 at physiological concentrations (Zheng et al., 1998). Prostatespecific antigen (PSA) is a kallikrien-like serine protease present in seminal plasma that causes proteolysis of IGFBP-3 and IGFBP-5, and PSA decreases IGFBP-3 induced cell growth inhibition (Cohen et al., 1992; 1994). Interestingly, IGFBP-3 in pregnancy serum, which appears fully proteolyzed when analyzed on SDS-PAGE, carries IGFs at normal concentration in the circulation, implying that its IGF-binding activity is unaffected (Suikkari and Baxter, 1992). Nevertheless, when stripped of its IGFs and tested for IGF-binding activity in vitro, a considerable reduction in affinity is evident (Lassare and Binoux, 1994).

\subsubsection{Dephosphorylation}

Phosphorylation is widely accepted to be the most important general mechanism for the regulation of protein function, affecting up to one third of all mammalian cell proteins, although mainly intracellular ones. Analysis of IGFBP-1 by anion-exchange chromatography and nondenaturing polyacrylamide gel electrophoresis has identified up to five IGFBP-1 variants, differing only in their degree of phosphorylation. Various cell types such as Hep G2, decidual, and liver cells were found to secrete predominantly phosphorylated forms, whereas amniotic fluid and fetal serum contained substantial amounts of nonphosphorylated and lesser-phosphorylated variants (Jones et al., 1991; 1995; Frost et al, 1991). The stimulation and inhibition of the IGF actions have been found in association with the nonphosphorylated and phosphorylated IGFBP-1 variants, respectively. Dephosphorylation of human (but not rat) IGFBP-1 renders it less able to bind IGF-I: the nonphosphorylated IGFBP-1 isoforms have reportedly four- to six-fold lower binding affinity for IGF-I as compared with the phosphorylated variants (Jones et al., 1991; 1995).

\subsection{IGF-independent actions of the IGFBPs}

IGF-I-independent, direct cellular actions have been demonstrated for IGFBP-1, -2, -3 and -5 . Jones and coworkers showed that IGFBP-1 stimulated the migration of Chinese hamster ovary $(\mathrm{CHO})$ and fibroblast cells by binding via its $\mathrm{C}$-terminal integrin 
receptor recognition $\mathrm{RGD}$ sequence to the cellular $\alpha 5 \beta 1$ integrin (fibronectin receptor) (Jones et al., 1993). However, the same interaction was shown to inhibit IGF-I stimulated as well as basal motility of breast cancer cell line MDA-231BO (Zhang et al., 2002). It is possible, that IGFBP-1 competes with fibronectin and blocks integrinmediated effect required for cell migration. The Arg-Gly-Asp motif in IGFBP-1 also appears to be involved in the induction of focal adhesion kinase dephosphorylation, cell detachment, and subsequent apoptosis, because IGFBP-1 and a synthetic ArgGly-Asp-containing peptide had similar effects in initiating these events in breast cancer cells (Perks et al., 1999). Thus, it appears that the effect of IGFBP-1 on integrin function could be cell specific. IGFBP-2, the only other IGFBP with RGD sequence, binds to $\alpha v \beta 3$ integrin and is localized efficiently to $\alpha v \beta 3$-expressing tumors. The interaction was found to mediate reduced migration and was associated with reduced in vivo tumor growth (Pereira et al., 2004).

Table 1. General characteristics of the human IGFBPs

\begin{tabular}{|c|c|c|c|c|c|c|}
\hline & IGFBP-1 & IGFBP-2 & IGFBP-3 & IGFBP-4 & IGFBP-5 & IGFBP-6 \\
\hline IGF preference & IGF-I $\geq$ IGF-II & IGF-II>IGF-I & $\mid$ IGF-I $\geq$ IGF-II & IGF-I>IGF-II & IGF-II>IGF-I & $|G F-||>>| G F-\mid$ \\
\hline Molecular weight & $25 \mathrm{kDa}$ & $31 \mathrm{kDa}$ & $43-45 \mathrm{kDa}$ & $24 \mathrm{kDa}$ & $29 \mathrm{kDa}$ & 28-30 kDa \\
\hline Serum concentration & $2-15 \mathrm{nM}$ & $2-15 \mathrm{nM}$ & $100 \mathrm{nM}$ & $2-15 \mathrm{nM}$ & $2-15 \mathrm{nM}$ & $2-15 \mathrm{nM}$ \\
\hline Binds ALS & & & + & & + & \\
\hline Nuclear target sequence & & & + & & + & \\
\hline Heparin binding residues & & & + & & + & + \\
\hline RGD sequence & + & + & & & & \\
\hline ECM binding & + & + & + & & + & + \\
\hline N-glycosylation & & & + & + & & \\
\hline O-glycosylation & & & & & + & + \\
\hline Serine phosphorylation sites & + & & + & & + & \\
\hline
\end{tabular}

Most studies on the IGF-independent actions of IGFBPs have focused on IGFBP3 and IGFBP-5. IGFBP-3 is an important cell growth inhibitor and a potential anticancer agent. It directly inhibits the proliferation of chicken embryo fibroblasts (Delbé et al., 1991), the estrogen receptor-negative human breast cancer cell line Hs578T (Oh et al., 1993) and prostate cancer cells (Cohen et al., 1993). Transfection of the IGFBP-3 gene into mouse fibroblasts decreases proliferation (Cohen et al., 1993). One of the initial key experiments demonstrated a growth inhibitory effect of overexpressed IGFBP-3 in fibroblasts isolated from IGF-IR-deficient mice (Valentinis et al., 1995). The protein directly induces apoptosis in a prostate cancer cell line, mediates at list in part 
the growth inhibitory actions of retinoic acid, TGF- $\beta$ and the tumor suppressor gene p53 (Oh et al., 1995; Leal et al., 1997; Rajah et al., 1997). Transforming growth factor $\beta$ and retinoic acid, which are known to inhibit cell growth and induce apoptosis in a variety of normal and malignant cell types, also induce the expression and secretion of IGFBP-3 (Martin et al., 1992; 1995, Babajko and Binoux, 1996). In 1995, Buckbinder and coworkers first identified IGFBP-3 as one of the p53-inducible genes (Buckbinder et al., 1995).

Although the precise mechanism of growth regulation by IGFBP-3 is unknown, one plausible explanation is that IGFBP-3 is transported to the nucleus where it directly or indirectly modulates gene transcription. The basic carboxyl-terminal domain of IGFBP-3 contains a region with strong sequence homology to nuclear localization signals (NLS) (Figure 4). In addition, this basic region shares homology with the DNAbinding domains of several transcription factors (Turner et al., 1989). Based on sequence homology between the basic carboxyl-terminal regions of IGFBP-3 and IGFBP-5 (IGFBP-3, amino acids 215-232; IGFBP-5, amino acids 201-218), IGFBP-5 also has the potential to be targeted to the nuclei of cells and to interact directly with DNA. Both IGFBP-3 and -5 have been indeed identified in the nuclei of opossum kidney cells ( $\mathrm{Li}$ et al., 1997), mink lung epithelial cells and T47D breast cancer cells (Schedlich et al., 1998).

Taylor and coworkers have isolated a $75 \mathrm{kDa}$ receptor which transports IGFBP-3 intracellularly into the $\alpha$-granules of megakaryocytes, and thus potentially makes it possible for the protein to reach cell nucleus (Taylor and Spencer, 2001). The same receptor is present on the breast cancer cells that respond to IGFBP-3 and absent on those that do not. Inhibition of receptor-mediated endocytosis does not however affect nuclear uptake of IGFBP-3, suggesting that it uses also an alternative non-classical import pathway for transport across the plasma membrane (Schedlich et al., 1998). Lee and coworkers observed cellular internalization of IGFBP-3 occurring via interaction with caveolin, one of two (next to clathrin) major proteins mediating endocytic uptake (Lee et al., 2004). Both IGFBP-3 and -5 possess caveolin docking domains in their C-domains (Figure 3).

The transforming growth factor- $\beta$ receptor $(T \beta R-V)$, a membrane glycoprotein with Ser-specific protein kinase activity, was found to be identical to IGFBP-3 receptor, which mediates IGF-independent growth inhibition (Leal et al., 1997; Leal et al., 1999; Wu et al., 2000). Findings of Huang and coworkers suggest that the growth inhibition 
results from dephosphorylation of insulin receptor substrates, IRS-1 and -2 , by the IGFBP-3-bound T $\beta$ R-V (Huang et al., 2004).

IGFBP-5 stimulates mitogenesis of endothelial, osteoblast and other cell types independent of IGF (Andress and Birnbaum, 1992; Andress et al., 1993; Andress, 1995). Cell association is required, but receptor has not been isolated. Fragments of the IGFBPs have been reported to have both inhibitory and stimulatory actions (Binoux et al., 1999). IGFBP-5(1-169) directly stimulates osteoblast proliferation suggesting a regulatory role in bone formation (Andress et al., 1992; Mohan et al., 1999). The Cterminal metal binding domain (MBD) of IGFBP-3 has been shown to mediate cellular uptake and nuclear localization of C-terminally conjugated not related partner proteins: green fluorescent protein (GFP) and horseradish peroxidase (HRP). A 12-amino acid long MBD peptide triggered apoptosis as effectively as an intact IGFBP-3 in chemically or nutritionally stressed human embryonic kidney (HEK) cells (Singh et al., 2004).

It has been also shown that the E7 protein encoded by human papillomavirus type 16 binds to intracellular IGFBP-3 and triggers its proteolytic cleavage, preventing its proapoptotic functions, hence overriding senescence (Mannhardt et al., 2000).

\subsection{The structure of the IGFBPs}

The molecular size of the six IGFBPs ranges from 22 to $32 \mathrm{kDa}$. The $\mathrm{N}$ - and C-terminal thirds of all the IGFBPs are homologous and heavily cross-liked by disulfide bonds whose positions are conserved (Figure 4). The middle regions have a minimal homology and only IGFBP-4 has a disulfide bond in this region. Secondary structure predictions and accessibility to proteolysis suggests that the linker region is exposed and lacks significant structure. Several studies aimed at establishing the disulfide bond linkages of IGFBPs using conventional biochemical methods. Only mapping of disulfides in IGFBP-6, containing six amino-terminal cysteine bridges, one less than other IGFBPs, produced complete and accurate map of all the linkages in both $\mathrm{N}$ - and C-domains (Neumann et al., 1999). In a study on IGFBP-4, Chelius and coworkers established 8 out of 10 disulfide bonds and narrowed the remaining assignment to 2 possibilities (Chelius et al., 2001). On the following pages of this thesis it will be unequivocally shown that the $\mathrm{N}$-terminal linkages identified by Chelius were incorrectly assigned. All IGFBPs examined so far (IGFBP-1, -2, -4, -6) have an identical intra 


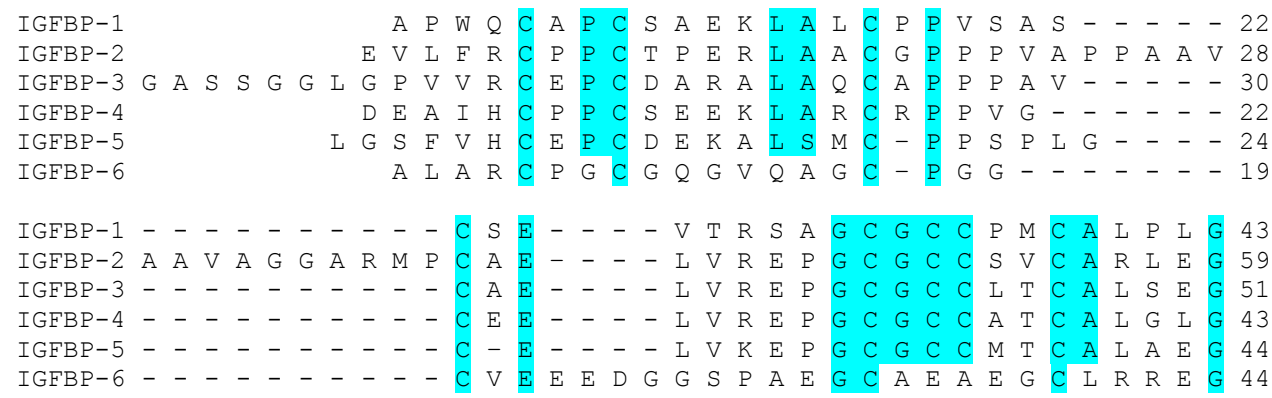

IGFBP-1 A A C G V A T A R C A R G L S C R A L P G E Q Q P I H A I T R G Q G A 78

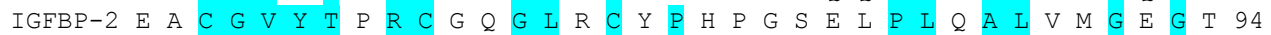
IGFBP-3 \& $\mathrm{P} C \mathrm{C}$ G I Y T E R C G S G I R C Q P S P D E A R P I Q A L L D G R G L 86

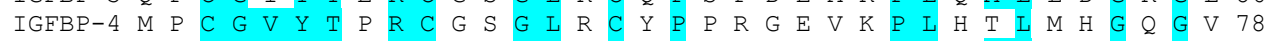
IGFBP-5 Q S C G V Y T E R C A Q G L L C L P R Q D E E K $P$ I I H A L L H G R G V 79 IGFBP-6 Q E C G V Y T P N C A P G L $Q$ C H P P K D D E A P L R A L L L G R G R 79

IGFBP-1 C V Q E S D A S A P H A A E A G S P E S P E S T E I T E E E L L D M F 113 IGFBP-2 C E K R R D A E Y G A S P E $Q$ V V A D N G D D H S E G G L V E N H V D S 129 IGFBP-3 C V N A S A V S R L R A Y L L P A P P A P G IGFBP-4 C M E L A E I E A I Q E S L Q P S D K D E G D H P N N S F S P C S A H 113

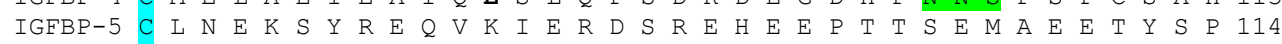

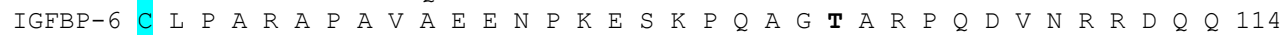

IGFBP-1 H L M A P S E E D H S I L W D A I S T Y D G S K A L H V T N I K K W K 148 IGFBP-2 T M N M L G G G G S A G R K P L K S G M K E L A V F R E K V T E $Q$ O H R 164 IGFBP-3 V E S P S V S S T H R V S D P K F H P L H S K I I I I K K G H A K D S 156

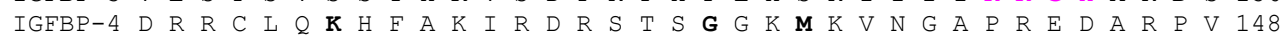
IGFBP-5 K I F R P K H T R I S E L K A E A V K K D R R K K L T Q S K F V G G A 149

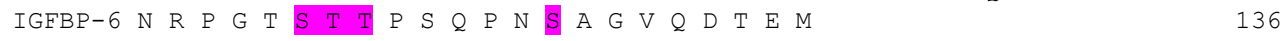

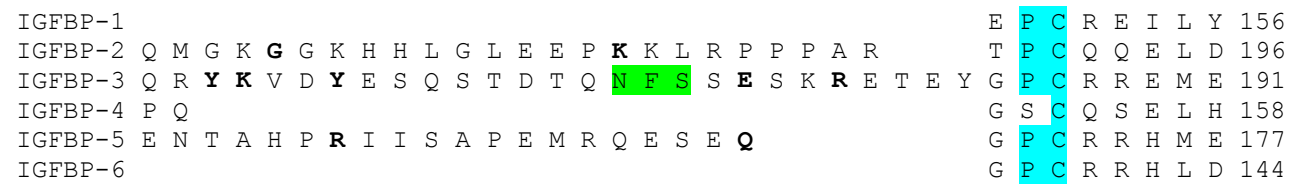

IGFBP-1 R V V E S L A K A Q E T S - - G E - E - I S K F Y L P N C N K N G F Y 187 IGFBP-2 Q V L E R I S T M R L P D E R G P L E H L Y S L H I P N C D K H G L Y 231 IGFBP-3 D T L N H L K F L N V L S V R G - - - V - - H I P N C D K K G F Y 219 IGEBP-4 R A L E R L A A S Q - - S - R T H - E D L Y I I P I P N C D R N G N F 189 IGFBP-5 A S L Q E L K A S E M R V P R A - - - V Y - - L P N C D R K G F Y 205 IGFBP-6 S V L Q Q L Q T E V Y - - R G - A Q T L Y - - V P N C D H R G F Y 172

IGFBP-1 H S R Q C E T S M D G E A G L C W C V Y P W N G K R I P G S P E I - R 221 IGFBP-2 N L K \& C K M S L N G Q R G E C W C V N P N T G K L I \& G A P T I - R 266 IGFBP-3 K K K Q C R P S K G R K R G F C W C V D K - G Q P L P G Y T T K G K 253 IGFBP-4 H P K Q C H P A L D G Q R G K C W C V D R K T G V K L P G - G L E P K 223 IGFBP-5 KR K Q C K P S R GRKR GICW C V D I - G M K L P G M - E Y V D 238 IGFBP-6 R K R Q C R S Q G Q R R G P C W C V D R M - GK S L P G S P D - G N 205

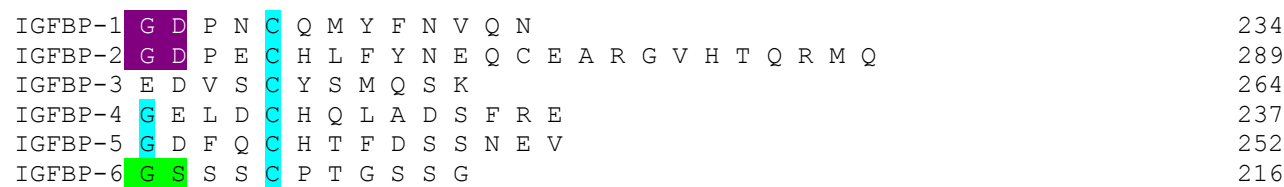

Figure 4. Sequence alignment of human IGFBP-1 to -6 . Conserved residues are indicated by cyan shading. Blue box - caveolin scaffolding docking domain (Lee et al., 2004); yellow nuclear localization sequences (NLS) (Schedlich et al., 1998); purple - potential integrinbinding sequences; green - potential N-glycosylation sites; magenta - identified O-glycosylation sites; red - phosphorylated serines (Jones et al., 1995; Hoeck et al., 1994), lettering in magenta - heparin-binding domains (Booth et al., 1995; Knudtson et al., 2001); underlined metal binding domain (MBD) (Singh et al., 2004). In bold - amino acids, after which cleavage occurs (Binoux et al., 1999). 
domain disulphide-bonding pattern of the C-domain (Forbes et al., 1998; Neumann et al., 1998; Standker et al., 2000). Regions of amino acid sequence homology and the conserved $\mathrm{N}$ - and C-terminal disulfide cross-linking pattern in IGFBPs suggest maintenance of a conserved structure across all IGFBPs in these regions. IGFBPs with reduced disulfides exhibit no IGF binding activity, suggesting the importance of the bridging for maintaining structural elements required for ligand binding (Landale et al., 1995; Hashimoto et al., 1997; Qin et al. 1998; Neumann and Bach, 1999).

Several properties of IGFBPs and IGFs, such as the intrinsic disorder of the central one-third of the IGFBPs, the highly disulfide bridged folded domains, and rapid aggregation of IGFs have hindered structural studies with these proteins (reviewed in Clemmons, 2001, in Hwa et al., 1999; in Carrick et al., 2002; Cooke et al., 1991; Torres et al., 1995). Only in the last few years have reports on structural characterization of small domains of IGFBPs appeared. The first direct information on IGFBP folding came from the NMR study of miniNBP-5, a short amino-terminal fragment spanning residues 40-92 (Kalus et al., 1998), which was later confirmed by X-ray crystallography (Zeslawski et al., 2001). The structure of this minimum-binding region of the N-terminal domain can be described as a compactly arranged, short threestranded antiparallel $\beta$-sheet (residues 46-47, 59-61, 78-82). As expected, the fold of miniNBP-5 determined by NMR was conserved in the crystal structure with IGF-I, although complex formation did rigidify the miniNBP-5 loop at residues 62-69.

Headey and coworkers have recently determined a solution structure of the carboxyl-terminal domain of IGFBP-6 (residues 161-240 of the full-length precursor) using NMR (Headey et al., 2004). The domain consists of a thyroglobulin type-1 fold comprising an $\alpha$-helix followed by a loop, a three-stranded anti-parallel $\beta$-sheet incorporating a second loop, and finally a disulfide bonded flexible third loop. The structure is in an agreement with a model proposed for C-terminal domain of IGFBP-4 (residues 136-237) based on the crystal structure of homologous protein, p41 (a proteolytic fragment of MHC class II) (Mazebourg et al., 2004).

\subsubsection{Posttranslational modifications}

IGFBPs 3-6 are glycosylated, but only IGFBP-3 is heavily glycosylated, which increases its molecular weight to $43-45$ from $28.7 \mathrm{kDa}$. On the basis of the number and molecular masses of the electrophoretic bands detected for each glycoform, there is approximately 4, 4.5 and $5 \mathrm{kDa}$ of carbohydrate on Asn89, Asn109 and Asn172 
respectively, with variable occupancy of Asn172. The three residues are located in the weakly conserved central region. Ternary complex formation by the three glycovariants in the presence of acid-labile subunit (ALS) and excess IGF-I is not significantly different from that of fully glycosylated recombinant human IGFBP-3. In contrast, Asn to Asp substitutions decrease ALS binding activity (Firth and Baxter, 1999). Glycosylation of IGFBP-3 is required for high-affinity binding to its receptor, but no other definitive function has been discerned for the sugar moieties on the other IGFBPs (Taylor et al., 2001).

As mentioned above, phosphorylation of IGFBP-1 increases its affinity for IGFs, but appears to have no affect on that of circulating IGFBP-3 (Jones et al., 1995) or IGFBP-5, although it does decrease the binding of IGFBP-3 to ALS and cell surfaces. IGFBP-3 is secreted from the liver as the phosphorylated form with subsequent dephosphorylation leading to a 2-fold increase in its affinity for the ALS (Forbes et al., 1998). Results of Schedlich and coworkers suggest that when IGF-I is cotransported into the nucleus by IGFBP-3, phosphorylation of IGFBP-3 by nuclear double-stranded DNA-dependent protein kinase (DNA-PK) provides a means for releasing bound IGF-I and creating a phosphoform of IGFBP-3 with increased affinity for nuclear components (Schedlich et al., 2003). There are reports on both stimulatory (Coverley and Baxter, 1995) and inhibitory (Mishra and Murphy, 2003) effects of IGF on IGFBP-3 phosphorylation.

\subsection{IGF - IGFBP interaction}

\subsubsection{IGF residues involved in IGFBP binding}

The earliest data came from studies of IGF-I naturally truncated at the amino terminus, DES-(1-3)-IGF-I, which binds to IGFBP-3 with several times lower affinity than natural IGF-I and shows greatly reduced binding to other IGFBPs (Forbes et al., 1988). Mutational analysis showed Glu3 to be an important determinant of binding, because variants like [Arg3]IGF-I and [GIn3, Ala4]IGF-I show considerably reduced IGFBP interaction. Other important IGFBP-binding determinants of IGF-I, as revealed by mutagenesis experiments, include GIn15 and Phe16 in the B domain of IGF-I and the A-domain residues Phe49, Arg50, and Ser51, because substitution of these for the corresponding residues in insulin considerably reduces IGFBP binding (Clemmons et 
al., 1990). In IGF-II, mutation of Phe26 in the B domain has a pronounced effect on binding to all six of the IGFBPs, most notably IGFBP-1, and, as with IGF-I, the residues Phe48, Arg49, and Ser50 are also of key importance (Bach et al., 1993).

\subsubsection{IGFBP residues involved in IGF binding}

The structural basis for the IGF binding by IGFBPs has been probed for past 20 years by mutagenesis studies (reviewed in: Clemmons, 2001). Mutagenesis of both aminoand carboxyl-terminal residues has been shown to disrupt binding, but it is not always clear whether these loss-of-function mutations affect gross IGFBP structure or point to specific interacting residues. It is generally accepted, however, that the primary IGFbinding site is located in the N-terminal region: deletions of amino-terminal residues and point mutations of residues in this region, particularly between conserved Cys residues 9 and 12, produced IGFBPs with no or reduced affinity to IGF. The direct evidence for an amino-terminal IGF-binding domain came from the solution structure of a recombinant IGFBP-5 fragment determined by Kalus and coworkers and later confirmed by Zeslawski and coworkers using X-ray crystallography. The studies identified a key role for IGFBP-5 residues Val49, Tyr50, Pro62, and Lys68-Leu74 in IGF binding.

Mutagenesis and functional studies also indicated that carboxyl-terminal residues were important in IGF binding. Removal of most or the entire C-terminal domain of human IGFBP-3 (Firth et al., 1998), IGFBP-4 (Rajah et al., 1997, Qin et al., 1998), or IGFBP-5 (Andress et al., 1993) disrupts IGF binding, although some residual activity is typically seen. In IGFBP-4, Cys205 through Val214 have been described as key residues (Qin et al., 1998), whereas in bovine IGFBP-2, a more amino-terminal sequence (Lys222-Asn236, corresponding to residues 185-199 of human IGFBP-4) was found to be important (Forbes et al., 1998). The IGF-II binding site on the Cdomain of IGFBP-6 was defined as a largely hydrophobic surface patch involving the $\alpha$-helix (residues Pro162 to Thr176), the first $\beta$-strand (Leu185 to Asp191) and the first and second loops.

The issue of the structural determinants required for high affinity binding of IGF-I by IGFBPs and its implications for interaction of IGF-I with IGF-IR will be addressed in the Discussion section of this thesis. 


\subsection{The IGF system and cancer}

Most cultured cancer cells express increased levels of components of the insulin-like growth factor system (reviewed in: LeRoith and Roberts, 2003). Many investigators have reported an association between serum concentrations of IGF-I and increased risks of breast, prostate, colorectal, and lung carcinomas (reviewed in: Furstenberger and Senn, 2002). The link between cancer and IGF signaling is also consistent with recent epidemiological studies showing an increased relative risk for the development of colon, prostate, breast, lung, and bladder cancers in individuals with circulating IGF-I levels in the upper quartile of the normal range (Chan et al., 1998). These findings were confirmed in animal models, where reduced circulating IGF-I levels result in significant reductions in cancer development, growth, and metastases, whereas increased circulating IGF-I levels are associated with enhanced tumor growth (Wu et al., 2003).

Characteristic alterations detected in primary hepatocellular (HCC) and cutaneous carcinomas, breast carcinomas and hepatoma cell lines, comprise the overexpression of the IGF-IR (sometimes accompanied by its hyperphosphorylation), emerging as critical event in malignant transformation and growth of tumors (Sharf and Braulke, 2003, Keehn et al., 2004). Simultaneous reduction of IGFBPs expression and the increase in proteolytic cleavage of IGFBPs result in an excess of bioactive IGFs. Defective functions of the IGF-II/mannose 6-phosphate receptor involved in degradation of IGF-II and the lysosomal targeting of cathepsin proteases capable to degrade extracellular matrix proteins may also contribute to the development of many human neoplasms (Sharf and Braulke, 2003; LeRoith and Roberts, 2003).

IGF-IR is overexpressed in many diverse tumor types and is a critical signaling molecule for tumor cell proliferation and survival. Moreover, IGF-IR also plays a critical role in cell transformation that is induced by tumor-virus proteins and oncogene products. Therapeutic strategies targeting the IGF-IR by eliminating it from the cell membrane, blocking the interaction with IGFs, or interrupting the signal transduction pathway downstream of IGF-IR, may therefore be an effective broad-spectrum anticancer action (Yu and Rohan, 2000; LeRoith et al., 1995).

Preventing the IGF-IR interaction with its ligand can be achieved either by neutralization of circulating IGFs or blocking the IGF binding site on IGF-IR. The strategies aiming at elimination of active IGFs utilize either specific antibodies or 
inhibitory IGFBPs (Arteaga et al., 1989; Firth and Baxter, 2002). Blocking of the active site of IGF-IR using antibodies has been extensively studied (Arteaga et al., 1989; Burtrum et al., 2003). Antibody-mediated blockade of ligand binding to the IGF-IR inhibited downstream signaling of IGFs pathways and, as a result, the mitogenic and proliferative potential of IGF-I and IGF-II were significantly reduced. In addition, some antibodies were shown to induce IGF-IR internalization and degradation, resulting in a significant reduction in tumor cell surface receptor density.

Direct reduction of IGF-IR expression has been approached by using antisense strategy through either oligonucleotides (Grzmil et al., 2004), stable transfections, or siRNAs (Neuenschwander et al., 1995; reviewed in: Baserga, 1995). Selective elimination of cells bearing the type I IGF receptor can be achieved using a chimeric toxin in which the cell-binding domain of Pseudomonas exotoxin was replaced with IGF-I (Prior et al., 1991). Blocking of the IGF-IR tyrosine kinase activity with a small molecule inhibitor would stop the downstream signal cascades. Certain compounds of the group cyclolignans have recently been reported to be potent and selective inhibitors of tyrosine phosphorylation of the IGF-IR (Girnita et al., 2004).

The IGF system plays a central role in many aspects of tumorigenesis. The approaches to its control listed above have numerous drawbacks and problems, including lack of specificity, difficulty of drug delivery, etc. A better understanding of this complex system will facilitate the development of novel approaches to diagnose and treat various human cancers. 


\section{Goals of the study}

While IGFBPs have different actions, all IGF-dependent actions, positive or negative, depend on binding to a single high-affinity binding site on IGFBPs. Therefore, elucidation of structural determinants of various IGFBPs in IGF binding is important to a general understanding of the biology of the IGF system and may shed light on how different IGFBPs exhibit specific actions.

A precise model of the IGF/IGFBP interaction can be created based on highresolution 3-D structures of the interacting proteins, kinetic data from binding studies on truncated variants and information on their dynamic behaviour from NMR experiments. The rationale behind this project is that determining the 3-D structure of the entire IGFBP would greatly improve our understanding of the structure-function relationship of this multi-dimensional protein family. Knowledge of structural determinants of the IGF binding site would place at our disposal a means to regulate the actions of the IGF, arguably one of the most important growth factors. This could be carried out by selectively modifying the binding affinity of IGFBPs for IGFs using site-directed mutagenesis on IGFBPs and/or IGFs. These mutants could serve as tools to investigate the biology of IGFs. Less active mutants would improve delivery of IGF-I to tissues. Mutants with increased affinity would find immediate use for blocking IGFs' activity. Design and in silico modeling of novel, low molecular weight ligands with therapeutic potential should be possible based on new interaction sites revealed in this study. 


\section{Techniques of structure and function analysis}

\subsection{X-ray crystallography}

\subsubsection{Crystal space groups and symmetries}

Crystals are by definition regular, three-dimensional arrays of atoms, ions, molecules or molecular assemblies. The minimal structural part that repeats in all three dimensions to build up the crystal is defined as the unit cell. Within the unit cell, a crystal can contain further symmetry elements, dividing it into several asymmetric units, which form the most basic structural element, which is related to all other identical objects in the unit cell by symmetry operations. The geometry of the unit cell together with the possible symmetry operations defines the space group of the crystal. Proteins and nucleic acids do not crystallize in space groups with inversion symmetries because they are composed of enantiomers (L-amino acids and D-sugars, respectively). As a consequence, the number of possible space groups is reduced from 230 to 65 , which are distributed between 7 crystal systems: triclinic, monoclinic, orthorhombic, tetragonal, trigonal, hexagonal and cubic. The combination of the 4 crystal lattices: primitive $(P)$, body centred $(I)$, face centred $(F)$ and centred in the $(010)$ planes $(C)$ with the mentioned 7 crystal systems allows a total of 14 Bravais lattices. Space groups containing translational elements, i.e., nonprimitive lattices, screw axes and glide planes, yield diffraction patterns in which certain classes of reflections are absent. Such systematic absences are termed space-group extinctions. Therefore, looking at the diffraction pattern it is often possible to determine the space group unambiguously. The knowledge of the crystal space-group makes possible to take advantage of the crystals symmetry - the higher the symmetry, the less data is required to be collected.

\subsubsection{Crystallization}

The first prerequisite for solving the three-dimensional structure of a protein by $\mathrm{X}$-ray crystallography is a well-ordered crystal that will diffract $X$-rays strongly. Crystallization of macromolecules is a multiparametric process involving three main steps: nucleation, growth and cessation of growth. It is indispensable for crystallization to bring the 
protein to a supersaturated state, which will force the macromolecules into the solid state - the crystal.

The Debye-Hückel theory describes how the solubility of the protein depends on ions. At low ionic strength the protein solubility increases with higher salt concentrations, this phenomenon is called salting-in. On the contrary, in the salting-out phenomenon, the protein at high ionic strength behaves as a neutral dipole and mainly hydrophobic effects govern solubility. Thus, salting-out corresponds to a decrease of protein solubility at high salt concentration. The crystallographer can shift the equilibrium from solution to supersaturation by reducing or, much more often, increasing the ionic strength of the protein solution. The main parameters that influence protein solubility are temperature, $\mathrm{pH}$ and presence of precipitating agents: salts, polymers or organic solvents. Crystal growth can be divided into two steps. First, a spontaneous nucleus formation occurs in the supersaturation area followed by formation of small ordered aggregates. After the critical amount of aggregated molecules (10-200) is surpassed, the crystal growth becomes an energetically privileged process. Crystal growth always needs a lower degree of supersaturation than nucleus formation. Crystals should grow slowly enough to achieve the maximum possible internal order.

There are several techniques for setting up crystallization experiments including sitting drop vapor diffusion, hanging drop vapor diffusion, sandwich drop, batch, microbatch, under oil, microdialysis, and free interface diffusion. Sitting and hanging drop methodologies are the most popular because they are easy to perform, require a small amount of sample, and allow large amount of flexibility during screening and optimization. Using the sitting drop technique one places a small ( 1 to $40 \mu \mathrm{l}$ ) droplet of the sample mixed with crystallization reagent on a platform in vapor equilibration with the reagent. The initial reagent concentration in the droplet is less than that in the reservoir. The volume proportion of mixed solutions is actually yet another variable in the crystallization experiment setup. Over time the reservoir solution pulls water from the droplet in a gaseous phase, and equilibrium establishes between the drop and the reservoir. During this equilibration process the sample is concentrated, increasing the relative supersaturation of the sample in the drop. The advantages of the sitting drop technique include speed and simplicity. The disadvantages are that crystals can sometimes adhere to the surface making removal difficult. This disadvantage can turn 
into an advantage where occasionally the surface of the sitting drop can assist in nucleation. The sitting drop is an excellent method for screening and optimization.

\subsubsection{X-ray diffraction}

When the primary beam from an X-ray source strikes the crystal, most of the X-rays travel straight through it. Some, however, interact with the electrons on each atom and cause them to oscillate. The oscillating electrons, as any oscillating charges, emit radiation serving as a new source of $X$-rays, which are emitted in almost all directions. We refer to this rather loosely as scattering. When atoms and hence their electrons are arranged in a regular three-dimensional array, as in a crystal, the X-rays emitted from the oscillating electrons interfere with one another. In most cases, these X-rays, colliding from different directions, cancel each other out; those from certain directions, however, will add together to produce diffracted beams of radiation that can be recorded as a pattern on a detector. Diffraction by a crystal can be regarded as the reflection of the primary beam by sets of parallel planes. The relationship between the reflection angle, $\theta$, the distance between the planes, $d$, and the wavelength, $\lambda$, is given by Bragg's law:

$$
2 \mathrm{~d} \sin \theta=\lambda
$$

Each atom in a crystal scatters X-ray in all directions, and only those that positively interfere with one another, according to Bragg's law, give rise to diffracted beams that can be recorded as a distinct diffraction spot. Each diffraction spot is the result of interference of all X-rays with the same diffraction angle emerging from all atoms. All heavy enough atoms contribute to the intensity of each spot, even though they do not lay on the Bragg's planes, from which the diffraction occurs. For a typical protein crystal, myoglobin, each of the about 20000 diffracted beams that have been measured contains scattered X-rays from each of the around 1500 atoms (not including hydrogen) in the molecule. To extract information about individual atoms from such a system the Fourier transform is employed.

\subsubsection{Solving the "phase problem"}

Each diffracted beam, which is recorded as a spot on the detector, is defined by three attributes: the amplitude, which can be measured from intensity of the spot; the wavelength, which is set by the X-ray source; and the phase, which is lost in X-ray experiments. All three properties for all of the diffracted beams have to be known to 
determine the position of the atoms. This is a so-called phase problem in X-ray crystallography. Several approaches are applicable to circumvent the phase problem: molecular replacement (MR), single isomorphous replacement (SIR), multiple isomorphous replacement (MIR), multiple-wavelength anomalous dispersion (MAD), and direct methods.

The method of Molecular Replacement (MR) depends on the availability of a sufficiently homologous model structure. That is, if the structure of a protein (search molecule) is known that is homologous to the crystallized protein (target molecule) with unknown structure, the former can be used as a model to calculate a starting set of phases that can be interactively refined (Hoppe, 1957; Huber, 1965). If successful, a preliminary model of the target structure will be obtained by correctly orienting and positioning the search molecule in the target cell. This solution can be optimised by rigid body refinement. Finally, the target structure can be put through cycles of map calculation, model fitting and refinement, which help to reduce the bias introduced by the starting model. Even in the absence of a suitable search molecule, the self-rotation function can be used to determine the direction and nature of non-crystallographic symmetry elements.

There are two operations in molecular replacement and these are known as the rotation and translation functions. In the rotation step, the spatial orientation of the known and unknown molecule with respect to each other is determined, while in the next step the translation which is needed to superimpose the now correctly oriented molecule onto the other is calculated. The basic principle of the MR method can be understood with help of the Patterson function of a protein crystal structure. The Patterson map is a vector map: distances between atoms in the real structure show up as vectors from the origin to maxima in the Patterson map. If the pairs of atoms belong to the same molecule, then the corresponding vectors are relatively short and their end-points are found not too far from the origin in the Patterson map; they are called self-Patterson vectors. If there were no intermolecular vectors (cross-Patterson vectors), this inner region of the Patterson map would be equal for the same molecule in different crystal structures, apart from a rotation difference. For homologous molecules it is not exactly equal but very similar. Therefore, the self-Patterson vectors can supply us with the rotation relationship between the known and the unknown molecular structures. The solutions of the rotation and translation functions are not always found in a straightforward way. In some cases, it can be necessary to modify 
the model, for instance, by ignoring the side chains and deletions/insertions in the model, and/or by systematically varying the resolution range of the X-ray data used in the search. With the rapid increase in the number of successful protein structure determinations, molecular replacement has become an extremely used technique for protein phase angle determination.

Without any previous knowledge of the structure multiple isomorphous replacement (MIR) is still the most commonly used method. Following heavy-metal introduction into the crystal lattice, some spots measurably increase in intensity, others decrease, and many show no detectable difference. The intensity differences can be used to deduce the positions of the heavy-atoms in the crystal unit cell. Fourier summations of these intensity differences give maps of the vectors between the heavy atoms, the so-called Patterson maps. From these vector maps, the atomic arrangement of the heavy atoms can be deduced. From the positions of the heavy metals in the unit cell, one can calculate the amplitudes and phases of their contribution to the diffracted beams of the protein crystals containing heavy metals. Subsequently the phase of the protein can be calculated.

Phase information can also be obtained by multiwavelength anomalous diffraction (MAD) experiments. For certain X-ray wavelengths, the interaction between the X-rays and the electrons of an atom causes the electrons to absorb the energy of the X-ray. This causes a change in the X-ray scattering of the atom, called anomalous scattering. By collecting data at several wavelengths near the absorption edge of an element in the crystal, the phase information analogous to that from MIR is obtained The intensity differences obtained in the diffraction pattern by illuminating such a crystal by X-rays of different wavelengths can be used in a way similar to the method of MIR to obtain the phases of the diffracted beams. Since wavelength needs to be tuned and modulated, the MAD method requires access to synchrotron radiation.

The amplitudes and the phases of the diffraction data from the protein crystals are used to calculate an electron-density map of the repeating unit of the crystal. This map then has to be interpreted as a polypeptide chain with a particular amino acid sequence. The quality of the map depends on the resolution of the diffraction data, which in turn depends on how well-ordered the crystals are. The initial model will contain errors, which can be subsequently removed by crystallographic refinement of the model. In this process the model is changed to minimize the difference between the experimentally observed diffraction amplitudes and those calculated for a 
hypothetical crystal containing the model instead of the real molecule. This difference is expressed as an R factor, residual disagreement, which is 0.0 for exact agreement and around 0.59 for total disagreement. In real life $R$ factor values between 0.15 and 0.25 are regarded satisfactory. In refined structures at high resolution (around $2 \AA$ ) there are usually no major errors in the orientation of individual residues.

The introduction presented here was adapted from Branden and Tooze (1999). For more details about crystallization procedures refer to McPherson (1999), and for the basis of X-ray crystallography refer to Drenth (1994) or Giacovazzo (1992).

\subsection{NMR spectroscopy}

When protein molecules are placed in a strong magnetic field, the spin of their atoms aligns along the field. This equilibrium alignment can be changed to an excited state by applying radio frequency (RF) pulses to the sample. When the nuclei of the protein molecule revert to their equilibrium state, they emit RF radiation that can be measured. The exact frequency of the emitted radiation from each nucleus depends on the molecular environment of the nucleus and is different for each atom, unless they are chemically equivalent and have the same molecular environment. These different frequencies are obtained relative to a reference signal and are called chemical shifts. The nature, duration, and combination of applied RF pulses can be varied enormously, and different molecular properties of the sample can be probed by selecting the appropriate combination of pulses.

One-dimensional proton spectra of protein molecules contain overlapping signals from many hydrogen atoms because the differences in chemical shifts are often smaller than the resolving power of the experiment. This problem has been bypassed by designing experimental conditions that yield a two-dimensional NMR spectrum. The diagonal in such a diagram corresponds to a normal one-dimensional NMR spectrum. The peaks off the diagonal result from interactions between hydrogen atoms that are close to each other in space. By varying the nature of the applied RF pulses these offdiagonal peaks can reveal different types of interactions. A COSY (correlation spectroscopy) experiment gives peaks between hydrogen atoms that are covalently connected through one or two other atoms. An NOE (nuclear Overhauser effect) spectrum, on the other hand, gives peaks between pairs of hydrogen atoms that are 
close together in space even if they are from amino acid residues that are quite distant in the primary amino acid sequence.

Two-dimensional NOE spectra, by specifying which groups are close together in space, contain three-dimensional information about the protein molecule. It is far from trivial, however, to assign the observed peaks in the spectra to hydrogen atoms in specific residues along the polypeptide chain because the order of peaks along the diagonal has no simple relation to the order of amino acids along the polypeptide chain. This problem has been solved by sequential assignment. Each type of an amino acid has a specific set of covalently connected hydrogen atoms that will give a specific combination of cross-peaks. From the COSY spectrum, or similar through bond experiments (for example, TOCSY) it is therefore possible to identify the $\mathrm{H}$ atoms that belong to each amino acid residue and, in addition, determine the nature of the side chain of that residue. However, the order of these fingerprints along the diagonal has no relation to the amino acid sequence of the protein. The sequence-specific assignment, however, can be made from NOE spectra that record signals from $\mathrm{H}$ atoms that are close together in space. These signals in the NOE spectra make it possible to determine which fingerprint in the COSY spectrum comes from residue adjacent to the one previously identified. The introduction of three- and fourdimensional NMR experiments and the availability of ${ }^{13} \mathrm{C}$-, ${ }^{15} \mathrm{~N}$-labelled proteins allow one to assign the proton, nitrogen and carbon chemical shifts of proteins and protein complexes with molecular weights well above $25 \mathrm{kDa}$ and to determine their structures in solution.

The final result of the sequence-specific assignment of NMR signals is a list of distance constraints from specific hydrogen atoms in one residue to hydrogen atoms in a second residue. This list immediately identifies the secondary structure elements of the protein molecule because both $\alpha$ helices and $\beta$ sheets have very specific sets of interactions of less than $5 \AA$ between their hydrogen atoms. It is also possible to derive models of the three-dimensional structure of the protein molecule. However, usually a set of possible structures rather that a unique structure is obtained. NMR spectroscopy, which has the disadvantage of being very time consuming and restricted for small molecular weight proteins (up to $30 \mathrm{kDa}$ ) in protein structure determination, has, however, many advantages in comparison to X-ray crystallography, as it can be useful for dynamics studies and can provide us with many other useful information about the protein in the solution. NMR proved to be an efficient technique for studying 
protein/ligand interactions that is used for identifying and designing high-affinity binding ligands for proteins in pharmaceutical research. The method called "SAR by NMR" (Structure Activity Relationship by NMR) is based on monitoring chemical shift changes of amide protons and nitrogens, which accompany the titration of potential ligands to a solution of ${ }^{15} \mathrm{~N}$-labelled protein (Branden and Tooze, 1999).

\subsection{Isothermal titration calorimetry}

Isothermal titration calorimetry (ITC) is a thermodynamic technique for monitoring any chemical reaction initiated by the addition of a binding component, and has become the method of choice for characterizing biomolecular interactions. When substances bind, heat is either generated or absorbed. Measurement of this heat allows accurate determination of dissotiation constants $\left(K_{D}\right)$, reaction stoichiometry $(n)$, enthalpy $(\Delta H)$ and entropy $(\Delta S)$, thereby providing a complete thermodynamic profile of the molecular interaction in a single experiment. In ITC experiment, a syringe containing a "ligand" solution is titrated into a cell containing a solution of the "macromolecule" at constant temperature. When ligand is injected into the cell, the two materials interact, and heat is released or absorbed in direct proportion to the amount of binding. As the macromolecule in the cell becomes saturated with ligand, the heat signal diminishes until only background heat of dilution is observed.

In the binding studies presented here, the VP-ITC system (MicroCal) was used. It is a sensitive isothermal titration calorimeter that uses a cell feedback network (CFB) to differentially measure heat produced or absorbed between the sample and reference cell. Twin coin-shaped cells are mounted in a cylindrical adiabatic environment, and connect to the outside through narrow access tubes (Figure 5). A thermoelectric device measures the temperature difference between the two cells and a second device measures the temperature difference between the cells and the jacket. As chemical reactions occur in the sample cell, heat is generated or absorbed. The temperature difference between the sample and reference cells $\left(\Delta \mathrm{T}_{1}\right)$ is kept at zero by the addition of heat to the sample or reference cell, as appropriate, using the CFB system. The integral of the power required to maintain $\Delta T_{1}=0$ over time is a measure of total heat resulting from the process being studied. 


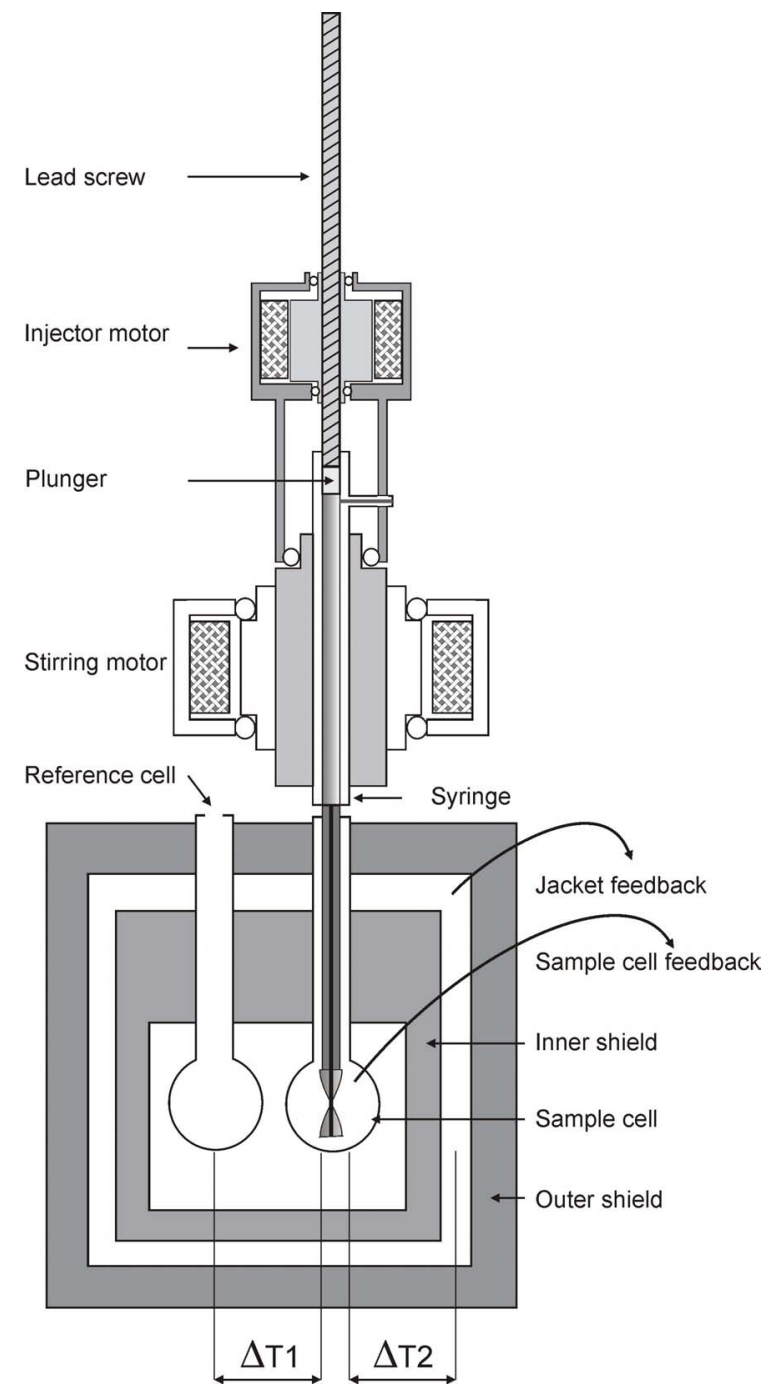

Figure 5. Schematic drawing of the VP-ITC microcalorimeter. A pair of identical coin shaped cells is enclosed in an adiabatic Outer Shield (Jacket). Access stems travel from the top exterior of the instrument to the cells. Both the coin shaped cells and the access stems are totally filled with liquid during operation. Temperature differences between the reference cell and the sample cell are measured and calibrated to power units. This generated signal is sometimes referred to as the "feedback" power used to maintain temperature equilibrium.

\subsection{Fluorescence spectroscopy}

Once a molecule has absorbed energy in the form of electromagnetic radiation, there are a number of routes by which it can return to the ground state - the statistically most common energy state for room temperature chemical species. Absorption of UV radiation by a molecule excites it from a vibrational level in the electronic ground state to one of the many vibrational levels in the electronic excited state. This excited state is 
usually the first excited singlet state. A molecule in a high vibrational level of the excited state will quickly fall to the lowest vibrational level of this state by losing energy to other molecules through collision. Fluorescence occurs when the molecule returns to the electronic ground state, from the excited singlet state, by emission of a photon. It is a dynamic phenomenon and the lifetime of the excited state is sufficient for a variety of chemical and physical reactions to take place prior to emission. The usual nanosecond time-window of fluorescence is normally shorter than that of other dynamic techniques (e.g. NMR). Fluorescence spectroscopy is essentially a probe technique sensing changes in the local environment of the fluorophore, which distinguishes it from generalized techniques, such as calorimetry, far-ultraviolet circular dichroism (CD), and infrared (IR) spectroscopy. Intensity of fluorescence emission measured as a function of wavelength comprises the fluorescence spectrum. The position of the emission spectrum reflects changes in energetics of the excited and ground state between excitation and emission of a photon (Ladokhin, 2000).

Fluorescence from the amino acid tryptophan has long been known to be sensitive to the polarity of its local environment (Burstein et al., 1973; Lakowicz, 1999; Vivian and Callis, 2001). Among the properties used are changes in the fluorescence intensity, wavelength maximum $(\lambda \max )$, band shape, anisotropy, fluorescence lifetimes, and energy transfer. They are applied to folding/unfolding, substrate binding, external quencher accessibility, etc. The power of this probe has been considerably amplified by the finding that Trp can often be substituted for other amino acids by sitedirected mutagenesis, with minimal effect on structure and activity. Trp $\lambda$ max is quite sensitive to its local environment, ranging from $308 \mathrm{~nm}$ (azurin) to $355 \mathrm{~nm}$ (e.g., glucagon) and roughly correlates with the degree of solvent exposure of the chromophore. Formation of various exciplexes (complexes in the excited state) and subsequent dipole relaxation is believed to cause the red-shift of emission. There are five most probable spectral forms of tryptophan residues according to the model of discrete states of Burstein et al. (1973) (Figure 6):

Spectral form A corresponds to emission of the unperturbed indole chromophore in the extremely nonpolar environment inside the protein globule.

Spectral form $S$ corresponds to the emission of the indole chromophore located in the relatively nonpolar environment inside the protein globule and forming a $1: 1$ exciplex with polar protein groups. 
Spectral form I corresponds to the emission of the indole chromophore in a somewhat polar but perhaps rigid environment inside the protein globule, forming a 2 : 1 exciplex with two neighboring polar protein groups. The fluorescence spectrum of class I has a maximum at about $330 \mathrm{~nm}$, width of about $50 \mathrm{~nm}$. A class I spectrum is observed for actin and chymotrypsin.

Spectral form // corresponds to emission of the indole chromophore at the protein surface. The chromophore is assumed to be in contact with bound water and other polar groups. The position of the maximum is at about $340 \mathrm{~nm}$ and the width is about $55 \mathrm{~nm}$. Many proteins contain tryptophan residues of this class, e.g. human serum albumin and myosin.

Spectral form III corresponds to the emission of the indole chromophore at the protein surface in contact with free water molecules. The spectrum of class III, with its maximum at about $350 \mathrm{~nm}$ and width of about $60 \mathrm{~nm}$, nearly coincides with the spectrum of free tryptophan. Tryptophan residues of spectral class III very seldom occur in the native proteins, but are typical for unfolded states.

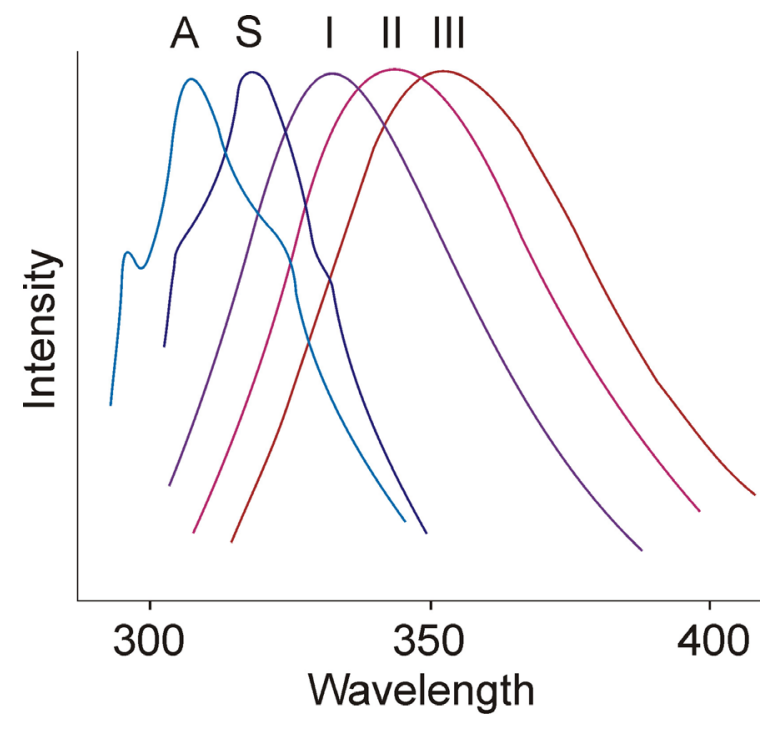

Figure 23. Normalized fluorescence spectra of tryptophan residues belonging to five spectral classes A, S, I, II and III according to Burstein (1973). 


\section{Materials and laboratory methods}

\subsection{Materials}

\subsubsection{E. coli strains and plasmids}

Cloning strains

XL1-Blue

Stratagene (USA)

One Shot TOP10

Invitrogen (Holland)

$\mathrm{DH} 5 \alpha$

Novagen (Canada)

Protein expression strains

One Shot ${ }^{\circledR}$ BL21 Star ${ }^{\mathrm{TM}}$

Invitrogen (Holland)

One Shot ${ }^{\circledR}$ BL21 Star ${ }^{\text {TM }}$ (DE3)

Invitrogen (Holland)

One Shot ${ }^{\circledR}$ BL21 Star ${ }^{\mathrm{TM}}$ (DE3) pLysS

Invitrogen (Holland)

Plasmids

pET 28a(+)

Novagen (Canada)

pQE 30

Qiagen (Germany)

pQE 80

Qiagen (Germany)

\subsubsection{Cell growth media and stocks}

\section{$L B$ medium}

For 1 liter LB medium:

$10 \mathrm{~g}$ bacto tryptone

$5 \mathrm{~g}$ bacto yeast extract

$10 \mathrm{~g}$ sodium chloride

$\mathrm{pH}$ was adjusted to 7.0. For the preparation of agar plates the medium was supplemented with $15 \mathrm{~g}$ agar.

For 1 liter TB medium

$12 \mathrm{~g}$ bacto tryptone

$24 \mathrm{~g}$ bacto teast extract

$10 \mathrm{~g}$ sodium chloride 
$4 \mathrm{ml}$ glycerol

$900 \mathrm{ml}$ deionized water

The medium was autoclaved, cooled; $100 \mathrm{ml}$ sterile $\mathrm{K}$ phosphate and glucose were added. The final concentration of glucose was $0.5 \%$

For 1 liter K-phosphate, $\mathrm{pH}$ 7.1: $\quad 23.1 \mathrm{~g} \mathrm{KH}_{2} \mathrm{PO}_{4}$

$125.4 \mathrm{~g} \mathrm{~K}_{2} \mathrm{HPO}_{4}$

Minimal medium (MM) for uniform enrichment with ${ }^{15} \mathrm{~N}$ :

For 1 liter MM:

$0.5 \mathrm{~g} \mathrm{NaCl}$

$1.3 \mathrm{ml}$ trace elements solution

$1 \mathrm{~g}$ citric acid monohydrate

$36 \mathrm{mg}$ ferrous citrate

$4.02 \mathrm{~g} \mathrm{KH}_{2} \mathrm{PO}_{4}$

$7.82 \mathrm{~g} \mathrm{~K}_{2} \mathrm{HPO}_{4} \times 3 \mathrm{H}_{2} \mathrm{O}$

$1 \mathrm{ml} \mathrm{Zn-EDTA} \mathrm{solution}$

$1 \mathrm{~g} \mathrm{NH}_{4} \mathrm{Cl}$ or ${ }^{15} \mathrm{NH}_{4} \mathrm{Cl}$

$\mathrm{pH}$ was adjusted to 7.0 with $\mathrm{NaOH}$, the mixture was autoclaved, upon cooling separately sterilized solutions were added: $25 \mathrm{ml}$ glucose, $560 \mu \mathrm{l}$ thiamin, antibiotics, $2 \mathrm{ml} \mathrm{MgSO} 4$ stock.

Defined medium for selective ${ }^{15} \mathrm{~N}$ labeling of proteins

For 1 litre of medium: $\quad 400 \mathrm{mg} \mathrm{Ala,} \mathrm{Gln,} \mathrm{Glu,} \mathrm{Arg,} \mathrm{Gly}$ $255 \mathrm{mg}$ Asp, Met

$125 \mathrm{mg}$ cytosine, guanosine, uracil 100 mg Asn, Leu, His, Lys, Pro, Thr, 100 mg Try

$400 \mathrm{mg}$ lle, Val

$50 \mathrm{mg}$ Phe, thymine, thymidine

$1.6 \mathrm{~g} \mathrm{Ser}$

$10 \mathrm{mg} \mathrm{CaCl} 2$

$2 \mathrm{~g} \mathrm{NaAc}$

$10 \mathrm{~g} \mathrm{~K}_{2} \mathrm{HPO}_{4}$ 
$1 \mathrm{~g}$ citric acid

$1.3 \mathrm{ml}$ trace element solution

$36 \mathrm{mg}$ ferrous citrate

$1 \mathrm{ml}$ Zn-EDTA solution

$1 \mathrm{~g} \mathrm{NH}_{4} \mathrm{Cl}$

$\mathrm{pH}$ was adjusted to 7.0 with $\mathrm{NaOH}$, the mixture was autoclaved. To the cooled medium, separately sterilized solutions were added: $25 \mathrm{ml}$ glucose, $560 \mu \mathrm{l}$ thiamin, antibiotics, $2 \mathrm{ml} 1 \mathrm{M} \mathrm{MgSO}_{4}$, sterile filtered:

50 mg Cys, Trp, nicotinic acid

$0.1 \mathrm{mg}$ biotin

$X \mathrm{mg}{ }^{15} \mathrm{~N}$-amino acid

Another portion of the ${ }^{15} \mathrm{~N}$-amino acid is added at the time of induction as well (same amount as added before, $0.22 \mu \mathrm{m}$ filtred).

\section{Stock solutions}

Glucose: $20 \%(w / v)$ in deionised $\mathrm{H}_{2} \mathrm{O}$, autoclaved;

Ampicillin: $100 \mathrm{mg} / \mathrm{ml}$ of ampicillin in deionised $\mathrm{H}_{2} \mathrm{O}$, sterilized by filtration, stored in aliquots at $-20^{\circ} \mathrm{C}$ until used. Working concentration: $200 \mu \mathrm{g} / \mathrm{ml}$.

Chloramphenicol was dissolved in ethanol $(0.5 \mathrm{~g} / 10 \mathrm{ml})$ to the end concentration of $50 \mathrm{mg} / \mathrm{ml}$. Working concentration: $50 \mu \mathrm{g} / \mathrm{ml}$.

Kanamycin: $50 \mathrm{mg} / \mathrm{ml}$ of kanamycin in deionised $\mathrm{H}_{2} \mathrm{O}$, sterile filtrated and stored in aliquots at $-20{ }^{\circ} \mathrm{C}$ until used. Working concentration: $100 \mu \mathrm{g} / \mathrm{ml}$.

IPTG: A sterile filtered $1 \mathrm{M}$ stock of IPTG in distilled water was prepared and stored in aliquots at $-20{ }^{\circ} \mathrm{C}$ until used.

Thiamin, $1 \%$, in deionised $\mathrm{H}_{2} \mathrm{O}$, sterilized by filtration.

$\mathrm{MgSO}_{4}, 1 \mathrm{M}$, in deionised $\mathrm{H}_{2} \mathrm{O}$, sterilized by filtration.

Zn-EDTA solution:

Trace elements solution:
$5 \mathrm{mg} / \mathrm{ml}$ EDTA

$8.4 \mathrm{mg} / \mathrm{ml} \mathrm{Zn(Ac)_{2 }}$

$2.5 \mathrm{~g} / / \mathrm{H}_{3} \mathrm{BO}_{3}$

$2.0 \mathrm{~g} / \mathrm{l} \mathrm{CoCl}_{2} \times \mathrm{H}_{2} \mathrm{O}$

$1.13 \mathrm{~g} / \mathrm{l} \mathrm{CuCl} 2 \times \mathrm{H}_{2} \mathrm{O}$ 
$9.8 \mathrm{~g} / \mathrm{l} \mathrm{MnCl} \mathrm{M}_{2} \times 2 \mathrm{H}_{2} \mathrm{O}$
$2.0 \mathrm{~g} / \mathrm{l} \mathrm{Na}_{2} \mathrm{MoO}_{4} \times 2 \mathrm{H}_{2} \mathrm{O}$
pH lowered with citric acid or $\mathrm{HCl}$.

\subsubsection{Protein purification - buffers}

Ion exchange and gel filtration chromatography buffers

Buffer $\mathrm{P}(0)$

$8 \mathrm{mM} \mathrm{KH}_{2} \mathrm{PO}_{4}$

$16 \mathrm{mM} \mathrm{Na}_{2} \mathrm{HPO}_{4}$

$0.05 \% \mathrm{NaN}_{3}$

$\mathrm{pH} 7.2$

Buffer P(1000)

$8 \mathrm{mM} \mathrm{KH}_{2} \mathrm{PO}_{4}$

$16 \mathrm{mM} \mathrm{Na}_{2} \mathrm{HPO}_{4}$

$1 \mathrm{M} \mathrm{NaCl}$

$0.05 \% \mathrm{NaN}_{3}$

$\mathrm{pH} 7.2$

PBS

$140 \mathrm{mM} \mathrm{NaCl}$

$2.7 \mathrm{mM} \mathrm{KCl}$

$10 \mathrm{mM} \mathrm{Na}_{2} \mathrm{HPO}_{4}$

$1.8 \mathrm{mM} \mathrm{KH}_{2} \mathrm{PO}_{4}$

$0.05 \% \mathrm{NaN}_{3}$

$\mathrm{pH} 7.3$

Buffers for IMAC under denaturing conditions

Buffer A

Buffer B
$6 \mathrm{M}$ guanidinium chloride

$100 \mathrm{mM} \mathrm{NaH}_{2} \mathrm{PO}_{4} \times \mathrm{H}_{2} \mathrm{O}$

$10 \mathrm{mM}$ Tris

$10 \mathrm{mM} \beta$-mercaptoethanol

$\mathrm{pH} 8.0$

$6 \mathrm{M}$ guanidinium chloride

$100 \mathrm{mM} \mathrm{NaH}_{2} \mathrm{PO}_{4} \times \mathrm{H}_{2} \mathrm{O}$ 
$10 \mathrm{mM}$ Tris

$10 \mathrm{mM} \beta$-mercaptoethanol

$\mathrm{pH} 6.5$

Buffer C

$6 \mathrm{M}$ guanidinium chloride

$100 \mathrm{mM} \mathrm{NaAc} \times 3 \mathrm{H}_{2} \mathrm{O}$

$10 \mathrm{mM} \beta$-mercaptoethanol

$\mathrm{pH} 4.0$

Buffer D

$6 \mathrm{M}$ guanidinium chloride

pH 3.0

Buffer E (refolding buffer)

$200 \mathrm{mM}$ arginine $\mathrm{HCl}$

1 mM EDTA

$100 \mathrm{mM}$ Tris

$2 \mathrm{mM}$ red $\mathrm{GSH}$

$2 \mathrm{mM}$ ox GSH

$10 \%(\mathrm{v} / \mathrm{v})$ glycerol

$0.05 \% \mathrm{NaN}_{3}$

$\mathrm{pH} 8.4$

Buffers for IMAC under native conditions

Binding buffer

$50 \mathrm{mM} \mathrm{NaH}_{2} \mathrm{PO}_{4}$

$300 \mathrm{mM} \mathrm{NaCl}$

$10 \mathrm{mM}$ imidazole

$\mathrm{pH} 8.0$

Wash buffer

$50 \mathrm{mM} \mathrm{NaH}_{2} \mathrm{PO}_{4}$

$300 \mathrm{mM} \mathrm{NaCl}$

$20 \mathrm{mM}$ imidazole

pH 8.0

Elution buffer

$50 \mathrm{mM} \mathrm{NaH}_{2} \mathrm{PO}_{4}$

$300 \mathrm{mM} \mathrm{NaCl}$ 
$250 \mathrm{mM}$ imidazole

$\mathrm{pH} 8.0$

Protease buffers

Buffer T (thrombin cleavage buffer)

$60 \mathrm{mM} \mathrm{NaCl}$

$60 \mathrm{mM} \mathrm{KCl}$

$2.5 \mathrm{mM} \mathrm{CaCl}_{2}$

$50 \mathrm{mM}$ Tris

$0.05 \% \mathrm{NaN}_{3}$

$\mathrm{pH} 8.0$

Buffer X (Factor Xa cleavage buffer)

$100 \mathrm{mM} \mathrm{NaCl}$

$4 \mathrm{mM} \mathrm{CaCl}_{2}$

$50 \mathrm{mM}$ Tris

$0.05 \% \mathrm{NaN}_{3}$

$\mathrm{pH} 8.0$

\subsubsection{Reagents and buffers for the SDS-PAGE}

Anode buffer (+):

Cathode buffer (-):

Separation buffer:

Stacking buffer:

Separation acrylamide:
$200 \mathrm{mM}$ Tris $\mathrm{pH} 8.9$

$100 \mathrm{mM}$ Tris $\mathrm{pH} 8.25$

$100 \mathrm{mM}$ tricine

$0.1 \%$ SDS

$1 \mathrm{M}$ Tris $\mathrm{pH} 8.8$

$0.3 \%$ SDS

$1 \mathrm{M}$ Tris $\mathrm{pH} 6.8$

$0.3 \%$ SDS

$48 \%$ acrylamide

$1.5 \%$ bis-acrylamide 
Stacking acrylamide:

$30 \%$ acrylamide

$0.8 \%$ bis-acrylamide

Pouring polyacrylamide gels

Separation gel:

$1.675 \mathrm{ml} \mathrm{H}_{2} \mathrm{O}$

$2.5 \mathrm{ml}$ separation buffer

$2.5 \mathrm{ml}$ separation acrylamide

$0.8 \mathrm{ml}$ glycerol

$25 \mu$ I APS

$2.5 \mu \mathrm{l}$ TEMED

Intermediate gel:

$1.725 \mathrm{ml} \mathrm{H}_{2} \mathrm{O}$

$1.25 \mathrm{ml}$ separation buffer

$0.75 \mathrm{ml}$ separation acrylamide

$12.5 \mu \mathrm{APS}$

$1.25 \mu$ I TEMED

Stacking gel:

$2.575 \mathrm{ml} \mathrm{H} \mathrm{O}_{2}$

$0.475 \mathrm{ml}$ stacking buffer

$0.625 \mathrm{ml}$ stacking acrylamide

$12.5 \mu \mathrm{l} 0.5 \mathrm{M}$ EDTA, pH 8.0

$37.5 \mu \mathrm{APS}$

$1.9 \mu$ TEMED

Protein visualization

Coomassie-blue solution:

$45 \%$ ethanol

$10 \%$ acetic acid

Destaining solution:

$5 \%$ ethanol

$10 \%$ acetic acid 


\subsubsection{Enzymes and other proteins}

$\begin{array}{ll}\text { BSA } & \text { New England BioLabs (USA) } \\ \text { CIP } & \text { New England BioLabs (USA) } \\ \text { BamH I } & \text { New England BioLabs (USA) } \\ \text { EcoR I } & \text { New England BioLabs (USA) } \\ \text { Hind III } & \text { New England BioLabs (USA) } \\ \text { Nde I } & \text { New England BioLabs (USA) } \\ \text { Xho I } & \text { New England BioLabs (USA) } \\ \text { Pfu turbo DNA Polymerase } & \text { Stratagene (USA) } \\ \text { Pwo DNA Polymerase } & \text { Roche (Germany) } \\ \text { T4 DNA Ligase } & \text { Roche (Germany) } \\ \text { Dpn I } & \text { Stratagene (USA) } \\ \text { Xa Factor } & \text { Sigma (USA) } \\ \text { Xa Factor } & \text { Novagen (Canada) } \\ \text { TAGzyme } & \text { Qiagen (Germany) } \\ \text { Thrombin } & \text { Sigma (USA) } \\ \text { Thrombin } & \text { Roche (Germany) } \\ \text { hIGF-I receptor grade } & \text { GroPep (Australia) }\end{array}$

\subsubsection{Kits and reagents}

Advantage 2 PCR kit

BD biosciences (USA)

ExSite Site-Directed Mutagenesis Kit Stratagene (USA)

ExCite Site-Directed Mutagenesis Kit Stratagene (USA)

QIAquick PCR Purification Kit Qiagen (Germany)

QIAprep Spin Miniprep Kit Qiagen (Germany)

QIAGEN Plasmid Midi Kit Qiagen (Germany)

QIAGEN Plasmid Maxi Kit Qiagen (Germany)

QuikChange Site-Directed Mutagenesis Kit Stratagene (USA)

Pre-Crystallization Test (PCT) Hampton Research (USA)

Rapid Ligation Kit Roche (Germany)

Complete Protease Inhibitor Cocktail Roche (Germany) 


\subsubsection{Protein and nucleic acids markers}

Mark12 Standard for SDS-PAGE Invitrogen (Holland)

Prestained Protein Marker, New England BioLabs (USA)

Broad Range (6-175 kDa) 1 kb DNA-Leiter Peqlab (Germany)

\subsubsection{Chromatography equipment, columns and media}

$\begin{array}{ll}\text { ÄKTA explorer 10 } & \text { Amersham Pharmacia (Sweden) } \\ \text { Peristaltic pump P-1 } & \text { Amersham Pharmacia (Sweden) } \\ \text { Fraction collector RediFrac } & \text { Amersham Pharmacia (Sweden) } \\ \text { Recorder REC-1 } & \text { Amersham Pharmacia (Sweden) } \\ \text { UV flow through detector UV-1 } & \text { Amersham Pharmacia (Sweden) } \\ \text { BioloLogic LP System } & \text { Biorad (USA) } \\ \text { HiLoad 26/60 Superdex S75pg } & \text { Amersham Pharmacia (Sweden) } \\ \text { HiLoad 10/30 Superdex S75pg } & \text { Amersham Pharmacia (Sweden) } \\ \text { Mono Q HR 5/5, 10/10 } & \text { Amersham Pharmacia (Sweden) } \\ \text { Mono S HR 5/5, 10/10 } & \text { Amersham Pharmacia (Sweden) } \\ \text { NiNTA-agarose } & \text { Qiagen (Germany) }\end{array}$

\subsection{Laboratory methods and principles}

\subsubsection{Constructs' design}

Careful choice of the constructs' amino and carboxyl termini may decide about later success or failure of crystallization attempts: erratically behaving, flexible parts of protein are known to inhibit crystallization or result in crystals of low quality, useless for structure determination. IGFBPs in turn are known to possess large unstructured regions, localized mainly in the central - linker - part, but also within the carboxylterminal domain.

Studies on domain organization of IGFBP-5, employing limited proteolysis, protein sequencing, mass spectrometry and NMR spectroscopy (Kalus, Rehm), determined approximate boundaries of the proteins' domains. Because of the high degree of sequence homology between IGFBPs, the estimates from IGFBP-5 studies could be 
extended and applied to the remaining members of the family, making the design of the constructs feasible. Thus, similarly to miniNBP-5(A40-192), miniNBP-4(A39-L82) was produced. NBP-4(A3-L82) and NBP-6(A1-A82) had their counterpart in 10kDNBP-5 (Kalus). For miniNBP-5, the last 9 amino acids showed no electron density in its IGF complex structure (Zeslawski et al., 2001) and were unstructured as determined by NMR (Kalus et al., 1998); equivalent residues were therefore initially not included in the constructs. It came later to our attention that IGFBP-4 residues Glu90 and Ser91 were probably imortnant for IGF-I and IGF-II binding (Qin et al. 1998), consequently the constructs were extended, resulting in NBP-4(D1-192) and NBP-6 (A1-S96). The residues Asp1 and Glu2 were also added to the NBP-4 N-terminus, when the importance of the amino-terminal residues became evident. The boundaries of carboxyl-terminal constructs were chosen assuming that amino acids in an immediate proximity of disulfide bridges have limited flexibility.

\subsubsection{Choice of the expression vector}

The present day existence of a large number of prokaryotic expression vectors, possessing various restriction sites and encoding tags facilitating fusion protein purification (Makrides, 1996), makes a choice of a suitable vector an easy task. The plasmid-encoded transcription signals: trascription initiation and termination sites and "strong" promoters, either hybrid or of phage origin, make high levels of mRNA synthesis possible. A long half-life of the mRNA is assured when specifically designed E. coli strains are used, for instance BL21 Star, characterized by deletions of genes encoding specific RNAses. The level of translation, which determines an overall yield of an overexpressed protein, depends on properties of the transcript RNA: its sequence and the quality of its codons, rather than on the strength of the promoter. The hairpin type secondary structure assumed by mRNA leads to attenuation of ribosome progression, greatly reducing protein production. The phenomenon of a "codon bias" arises from a different codon usage by eukaryotic and prokaryotic organisms: from the existing possible triplets encoding one amino acid, the former group of organisms sometimes chooses different ones than the latter. As a result, a pool of amino acyl-tRNAs and aa-tRNA synthetases present in E. coli differs from the one found in human cells. In particular, Arg codons AGA, AGG, CGG, CGA, lle codon AUA, Leu codon CUA, Gly codon GGA and Pro codon CCC are rarely used. This impact appears to be highest when these codons are present near the $\mathrm{N}$-terminus and 
when they appear consecutively. Several laboratories have shown that the yield of a protein whose genes contain rare codons can be dramatically improved when the cognate tRNA is increased within the host (Rosenberg et al., 1993). E. coli strains are commercially available that have artificially introduced copies of genes encoding the tRNAs, either at the genomic level or in a plasmid (Rossetta from Invitrogen; BL21 RP or RIL from Novagen).

The choice of the expression vector and fusion partner/tag is dictated by such parameters of the recombinant protein as its toxicity to $E$. coli and solubility. Tightly controlled expression systems are preferred, such as a pET system based on the phage T7 RNA polymerase, enabling a high level of control over a basal (i.e. uninduced) expression of genes encoding toxic proteins. The system provides several levels of control due to additional plasmids, which express T7 lysosyme, a natural T7 RNA polymerase inhibitor. Large, vector encoded fusion tags, such as glutathione Stransferase $(\mathrm{GSH})$, thioredoxin or maltose binding protein (MBP) often increase solubility and promote proper folding of recombinant fusion partners. For proteins directed into inclusion bodies, use of a 6-histidine fusion peptide (His-Tag) makes use of immobilized metal chromatography (IMAC) possible under strong denaturing/reducing conditions, enabling rapid purification. It is often observed that the presence of a tag sequence, even if it is a short His-Tag, preceding the recombinant gene, enhances its expression, increasing the products' yield. For carboxyl-terminal constructs of IGFBP-3 and IGFBP-6 lacking N-terminal tag sequences, expression yield was reduced below practical levels.

It is often required for functional studies of a recombinant protein and for crystallization purposes that the fusion tag is removed. This is usually achieved with an aid of a specific restriction protease. The enzymes most commonly used are serine proteases of the blood coagulation cascade: Factor $X a$ (cleaves after $R$ in a IEGRX sequence, $X \neq P$ ) and thrombin (cleaves after $R$ in LVPRGS). The sequences encoding the desired cleavage site are introduced by means of PCR, in 5'-overhangs of forward primers (Table 2). The specificity of the proteases often appears to be overestimated, and treatment of the fusion protein with these enzymes results in unwanted cleavage at sites possessing a certain degree of homology to the recognition sequences. Certain proteins are actually targets for blood circulating proteases, and are degraded even at low, physiological concentrations of the enzyme (Zheng et al., 1998). This problem can be completely avoided when amino dipeptidase (TAGzyme, Qiagen) is 
used. The enzyme cleaves dipeptides from the $\mathrm{N}$-terminus of a fusion protein, and stops before Arg, Lys or 1 or 2 residues before Pro. The enzyme is active in the presence of PMSF and EDTA, making it ideal for work with degradation susceptible proteins. The enzyme is used for removal of only short $\mathrm{N}$-terminal His-tags.

In choosing the restriction protease, enzyme - target protein compatibility has to be considered: the optimal cleavage conditions for the enzyme may not be appropriate for the stability of the protein in question. For instance, Factor $\mathrm{Xa}$ requires the presence of 1-4 $\mathrm{mM} \mathrm{Ca}^{2+}$ in the buffer and oxidizing conditions, and will not work in the presence of chelating agents, phosphate buffer, reducing agents or high salt. Presence of even small amounts of detergents may hamper its selectivity. PreScission protease on the other hand requires strong reducing conditions.

Plasmids pET 28a and PQE 80 were selected as vectors of choice for expression of the proteins discussed in this work. All amino-terminal constructs and mutants of IGFBP-4 and -5 were cloned into pET 28a in frame to a His/T7-tag of the following sequence: MGSSHHHHHHCCGLVPRGSHMASMTGGQQMGRGSIEGR. Treatment with Factor $\mathrm{Xa}$ (the cleavage site in bold) yielded proteins with native $\mathrm{N}$-termini. The same vector was used to express CBP-4, -5 and -6 , both Factor Xa and thrombin sites were introduced. The construct CBP-5 appeared to by remarkably susceptible to excessive cleavage by both restriction proteases. It was therefore subcloned into $\mathrm{PQE}$ 80 , enabling successful use of the TAGzyme.

\subsubsection{DNA techniques}

\subsubsection{Preparation of plasmid DNA}

The isolation of plasmid DNA from E. coli was carried out using dedicated plasmid purification kits from Qiagen. The kits employ a standard alkalic lysis of the precipitated bacteria in the presence of RNAse and strong ionic detergent, SDS, followed by neutralization/DNA renaturation with acetate. For purification, a crude cell lysate is loaded onto a silica gel column, washed with an ethanol-containing buffer, and eluted in a small volume, yielding up to $20 \mu \mathrm{g}$ of the plasmid DNA.

\subsubsection{PCR}

A polymerase chain reaction was employed to amplify desired DNA fragments and genes, introduce restriction sites, STOP codons and sequences encoding restriction 
protease cleavage sites. The primers were prepared according to standardized principles regarding the length, GC-content, melting temperature and occurrence of secondary structures of the hairpin type. All primers used for cloning and mutageneses are listed in Table 2. Three different kinds of recombinant thermostable DNA polymerases were used, each operating at slightly different conditions:

$\begin{array}{llll} & \text { Melting temp. } & \text { Annealing temp. } & \text { Synthesis temp. } \\ & 94^{\circ} \mathrm{C} & 55^{\circ} \mathrm{C} & 72^{\circ} \mathrm{C} \\ \text { Pfu Turbo } & 95^{\circ} \mathrm{C} & 55^{\circ} \mathrm{C} & 68^{\circ} \mathrm{C} \\ \text { Advantage } 2 & 94^{\circ} \mathrm{C} & 68^{\circ} \mathrm{C} & 68^{\circ} \mathrm{C}\end{array}$

Table 2. List of primers used in the work

\begin{tabular}{|c|c|c|}
\hline № & Name & Nucleotide sequence \\
\hline 1 & CBP3G184NcoF & CATGCCATGGGTCCCTGCCGTAGAGAAATGGAAGACACACTG \\
\hline 2 & CBP3BamQEF & CCCGGATCCCGCTATGGTCCCTGCCGTAGAGAAATGGAAGACACAC \\
\hline 3 & CBP3K267CHisR & ССССTCGAGCTTGCTCTGCATGCTGTAGCAGTGCACGTC \\
\hline 4 & CBP3K267HindR & CCСAAGCTTTCACTTGCTCTGCATGCTGTAGCAGTGCACGTCC \\
\hline 5 & $\operatorname{miniNBP} 4 \mathrm{XaF}$ & CGCGGATCCATTGAGGGTCGCGCCCTGGGCTTGGGGATGCCCTGCGG \\
\hline 6 & miniNBP4ThrF & CGCGGATCCCTGGTGCCGCGCGGCAGCGCCCTGGGCTTGGGGATGCCCTG \\
\hline 7 & miniNBP4R & CCCAAGCTTTCATTACAGCTCCATGCACACGCCTTGCCCG \\
\hline 8 & NBP4XaF & CGCGGATCCATTGAGGGTCGCGCCATCCACTGCCCGCCCTGC \\
\hline 9 & LNBP4D1XaF & CGCGGATCCATTGAGGGTCGCGACGAAGCCATCCACTGCCCGCCC \\
\hline 10 & NBP4APWXaF & CGCGGATCCATTGAGGGTCGCGCGCCGTGGCACTGCCCGCCCTGCTCCGAGGAG \\
\hline 11 & NBP4_BP3Xa1F & AGCAGCGGCGGCTTTGGTCCGGTGGTGCACTGCCCGCCCTGCTCCGAGGAGAAG \\
\hline 12 & NBP4_BP3Xa2F & CGCGGATCCATTGAGGGTCGCGGCGCGAGCAGCGGCGGCTTTGGTCCGGTGGTG \\
\hline 13 & LNBP4L92R & CCCCTCGAGTCATTACAGGCTTTCCTGGATGGCCTCGATCTC \\
\hline 14 & CBP4ThrG151F & CGCGGATCCCTGGTGCCGCGCGGCAGCTGCCAGAGCGAGCTGCACCGGG \\
\hline 15 & CBP4XaG151F & CGCGGATCCATTGAGGGTCGCTGCCAGAGCGAGCTGCACCGGG \\
\hline 16 & CBP4A232R & CCGCTCGAGTCATTACAGCTCCATGCACACGCCTTGCCCG \\
\hline 17 & CBP5ThrD135F & CGCGGATCCCTGGTGCCGCGCGGCAGCGACCGCAGAAAGAAGCTGACCCAG \\
\hline 18 & CBP5D247R & CCCAAGCTTTCATTAGTCGAAGGTGTGGCACTGAAAGTCCCC \\
\hline 19 & CBP5ThrG170F & CGCGGATCCCTGGTGCCGCGCGGCAGCGGCCCCTGCCGCAGACACATGGA \\
\hline 20 & CBP5XaG170F & CGCGGATCCATTGAGGGTCGCGGCCCCTGCCGCAGACACATGGA \\
\hline 21 & NBP6A1F & CGCGGATCCCTGGTGCCGCGCGGCTCCGCCTTGGCGCGGTGCCCAGGCTGC \\
\hline 22 & NBP6A82XHR & CССCTCGAGAGGCTTTCAGGCCGGAAGGCAGCGGCCTCGGCCG \\
\hline 23 & NBP6S96XHR & ССССTCGAGAGGCTTTCAACTCTCCTTAGGATTCTCCTCTGCAACAG \\
\hline 24 & CBP6ThrF & CGCGGATCCCTGGTGCCGCGCGGCTCCGGCCCATGCCGTAGACATCTGG \\
\hline 25 & CBP6XhoR & CCGCTCGAGTCATTACCCAGTGGGGCAGGAGGAGCTTCC \\
\hline 26 & CBP6HindR & CCCAAGCTTCCCAGTGGGGCAGGAGGAGCTTCCATTG \\
\hline 27 & CBP6NcolF & CATGCCATGGGCCCATGCCGTAGACATCTGG \\
\hline 28 & BP6fishoutF & CGCCTTGGCGCGGTGCCCAGGCTGGGGGCAAG \\
\hline 29 & BP6fishoutR & GCCGCTACTCCCAGTGGGGCAGGAGGAGCTTC \\
\hline
\end{tabular}




\begin{tabular}{lll}
\hline № & \multicolumn{1}{c}{ Name } & \multicolumn{1}{c}{ Nucleotide sequence } \\
\hline 30 & miniBP5L61YF & GGGGCTGCGCTGCTACCCCCGGCAGGACG \\
31 & miniBP5L61YR & CGTCCTGCCGGGGGTAGCAGCGCAGCCCC \\
32 & miniBP5L74MF & CGCTGCACGCCCTGATGCACGGCCGCGGGG \\
33 & miniBP5L74MR & CCCCGCGGCCGTGCATCAGGGCGTGCAGCG \\
34 & CBP4d168-174F & Pho-CTCTACTTCATCCCCATCCCCAACTGCGAC \\
35 & CBP4d168-174R & Pho-TGAAGCGGCCAGCCGCTCCAGCGCCCGGTG \\
36 & CBP4d167-177R & Pho-AGCGGCCAGCCGCTCCAGCGCCCG \\
37 & CBP4d167-177F & Pho-ATCCCCATCCCCAACTGCGACCGC \\
38 & CBP4d216-223F & Pho-GGGGAGCTGGACTGCCACCAGCTGGCTGAC \\
39 & CBP4d216-223R & Pho-GCCCCCCGGAAGCTTCACCCCCGTCTTCCG \\
40 & CBP4d213-225R & Pho-CGTCTTCCGGTCCACACACCAGCA \\
41 & CBP4d213-225F & Pho-CTGGACTGCCACCAGCTGGCTGAC \\
42 & CBP4W206R & CCGCTCGAGTCATTACCAGCACTTGCCACGCTGCCCATCCAGAGC \\
42 & NBP4H5XaF & CGCGGATCCATTGAGGGTCGCACTGCCCGCCCTGCTCCGAGGAGAAGC \\
43 & NBP4G5XaF & CGCGGATCCATTGAGGGTCGCGGCTGCCCGCCCTGCTCCGAGGAGAAGC \\
44 & NBP5A1XaF & CGCGGATCCAGCATTGAGGGTCGCCTGGGCTCCTTCGTGCACTGCGAGCCCTG \\
45 & NBP583HindXhoR & CCCAAGCTTCTCGAGTCATTCGTTGAGGCAAACCCCGCGGCCGTG \\
46 & NBP4G5XaF & CGCGGATCCATTGAGGGTCGCGGCTGCCCGCCTGCTCCGAGGAGAAGC \\
47 & NBP4cons3XaF1 & GCTGTGGGCCTGGGTCCGGTGGTGCGTTGCCCGCCCTGCTCCGAGGAGAAGCTG \\
48 & NBP4cons3XaF2 & CGCGGATCCAGCATTGAGGGTCGCGGTGCCGGCGCTGTGGGCCTGGGTCCGGTG \\
\hline
\end{tabular}

F-forward, R-reverse, Pho-5' phosphate group, restriction sites in bold, Stop codons in italics, encoded protease cleavage sites underlined.

\subsubsection{Digestion with restriction enzymes}

Usually, 1-2 units of each restriction enzyme were used per $1 \mu \mathrm{g}$ of plasmid DNA to be digested. The digestion was performed in a buffer specified by the manufacturer at the optimal temperature $\left(37^{\circ} \mathrm{C}\right)$ for $5-16 \mathrm{~h}$. The fragments ends that occurred after digestion were cohesive. To eliminate possibility of plasmid recirculation (possible when double-digestion does not occur with 100\% efficiency), 5'-ends of a vector were dephosphorylated using calf intestine phosphatase (CIP). CIP treatment was performed with 1 unit of enzyme per $3 \mu \mathrm{g}$ of plasmid DNA, at $37^{\circ} \mathrm{C}$ for $1 \mathrm{~h}$.

\subsubsection{Purification of $P C R$ and restriction digestion products}

DNA obtained from restriction digestion, phosphatase treatment or PCR was purified from primers, nucleotides, enzymes, buffering substances, mineral oil, salts, agarose, ethidium bromide, and other impurities, using a silica-gel column (QIAquick PCR Purification Kit, Qiagen). The QIAquick system uses a simple bind-wash-elute procedure. A binding buffer was added directly to the PCR sample or other enzymatic 
reaction, and the mixture was applied to the spin column. Nucleic acids adsorbed to the silica-gel membrane in the high-salt conditions provided by the buffer. Impurities and short fragments of single or double-stranded DNAs were washed away and pure DNA was eluted with a small volume of $10 \mathrm{mM}$ Tris $\mathrm{pH} 8.0$ or water.

\subsubsection{Mutagenesis of the miniNBP-5 and CBP-4}

Site directed mutagenesis of miniNBP-5 was performed by means of PCR, using enzymes and instructions supplied in the QuikChange Site-Directed Mutagenesis Kit (Stratagene). The mutagenic oligonucleotide primers were designed according to suggestions provided by the manufacturer: they possessed 30-32 bases in length, their melting temperatures were greater than $78{ }^{\circ} \mathrm{C}$, they had a $\mathrm{GC}$ content of more than $40 \%$ and terminated in $C$ or $G$ bases. The desired mutation was in the middle of the primer with $\sim 10-15$ bases of a correct sequence on both sides (Table 2, primers: 30 , $31,32,33)$. Vector pET 28a containing a copy of a gene encoding miniNBP-5 was used as a DNA template. High concentration of the template DNA and low number of PCR cycles, combined with high accuracy and fidelity of highly processive DNA polymerase Pfu Turbo, minimizes the occurrence of unwanted mutations.

The mutagenic PCR reaction mixture contained:

$\begin{array}{ll}10 \times \text { reaction buffer } & 5 \mu \mathrm{l} \\ \text { plasmid }(5 \mathrm{ng} / \mu \mathrm{l}) & 2 \mu \mathrm{l}(10 \mathrm{ng}) \\ \text { oligonucleotide primer } \mathrm{F} & 1.25 \mu \mathrm{l}(125 \mathrm{ng}) \\ \text { oligonucleotide control primerF } & 1.25 \mu \mathrm{l}(125 \mathrm{ng}) \\ \text { dNTP mix } & 1 \mu \mathrm{l} \\ \text { milli-Q to a final volume of } 50 \mu \mathrm{l} & 39.5 \mu \mathrm{l} \\ \text { PfuTurbo DNA polymerase }(2.5 \mathrm{U} / \mu \mathrm{l}) & 1 \mu \mathrm{l}\end{array}$

PCR cycling parameters:

denaturation:

$95^{\circ} \mathrm{C}, 30 "$

denaturation:

$95^{\circ} \mathrm{C}, 30^{\prime \prime}$

annealing:

$55^{\circ} \mathrm{C}, 1$ '

synthesis ( 1 min per 1000 base pairs):

$68^{\circ} \mathrm{C}, 4.5^{\prime}$ 
Following temperature cycling, the product was treated with $D p n \mathrm{I}\left(10 \mathrm{U}, 37^{\circ} \mathrm{C}\right.$, for 2 h). The $D p n$ I endonuclease (target sequence: $5^{\prime}-\mathrm{Gm}^{6} \mathrm{ATC}-3^{\prime}$ ) is specific for methylated and hemimethylated DNA and is used to digest the parental DNA template and to select for mutation-containing synthesized DNA. $2 \mu \mathrm{l}$ of the mixture were used to transform XL1-Blue or Top10 chemically competent cells. Plasmid DNA was isolated using QIAprep Spin Miniprep Kit (Qiagen) and was subjected to verification by automated sequencing.

Essentially the same procedure was applied for deletional mutagenesis of CBP-4. Since in this case a linear PCR product is obtained, 5'-phosphorylated primers were applied to enable a subsequent blunt-end ligation. After Dpn I treatment, linear DNA was end polished with cloned Pfu DNA polymerase to remove extended bases placed on the 3' ends of the PCR products by ExSite DNA polymerase. The DNA was then intramolecularly ligated at $37^{\circ} \mathrm{C}$ for $1 \mathrm{~h}$ with T4 DNA ligase and transformed into the XL1-Blue or Top10 supercompetent cells.

\subsubsection{Agarose gel electrophoresis of DNA}

For verification of the presence and length of PCR or restriction digestion products, agarose gel electrophoresis was performed. For this purpose $1 \%$ agarose in TBE buffer plus ethidium bromide was prepared. The solution was poured into a horizontal gel chamber to cool down and polymerise. The DNA samples were mixed with $6 x$ sample buffer prior loading. Electrophoresis was carried out at 100-120 V DC. Results were evaluated using UV illumination.

\subsubsection{Transformation of $E$. coli}

A number of factors have been elucidated that produced an increase in transformation efficiency. Such factors include: prolonged incubation of bacteria with $\mathrm{CaCl}_{2}$, addition of multiple cations, such as $\mathrm{Mg}^{2+}, \mathrm{Cs}^{2+}$ or $\mathrm{Rb}^{2+}$, into the transformation mixture and treatment of bacteria with dimethyl sulfoxide (DMSO), polyethylene glycol, hexaminecobalt, and dithiothreitol in the presence of both monovalent and divalent cations (Chung et al., 1989). After incubation with DNA, in order to make the cells keep the plasmid and to be certain they survive, the cells were heat shocked for several seconds to induce heat shock genes, which aid in survival and recovery. The cells were than incubated at $37^{\circ} \mathrm{C}$ without selective pressure; sufficient time was given for 
expression of antibiotic resistance genes. Plating on selective media enabled recovery of those cells that actually received the DNA.

Transformation by electroporation employs high voltage to temporarily puncture bacterial cell walls. High mortality of cells treated this way implicates the need for high concentrations of bacteria. Preparation of electrocompetent cells was resolved by thorough washing of pelleted cells in order to remove salt present in growth media, which would otherwise cause short-circuiting and mass destruction of the cells.

\subsubsection{Transformation of chemically competent cells}

$3 \mu \mathrm{l}$ of a ligation mix or ca. $50 \mu \mathrm{g}$ of plasmid DNA was added to $50 \mu \mathrm{l}$ of chemically competent cells. The mixture was incubated on ice for $30 \mathrm{~min}$ followed by a heat shock of $30 \mathrm{~s}$ at $42{ }^{\circ} \mathrm{C}$, short cooling on ice, and addition of $250 \mu$ of glucose and magnesium containing medium. After $1 \mathrm{~h}$ of incubation at $37^{\circ} \mathrm{C}, 20-50 \mu \mathrm{l}$ of the mixture was spread out on LB agar plates that included selective antibiotic and incubated overnight at 37 ${ }^{\circ} \mathrm{C}$.

\subsubsection{Transformation by electroporation}

$1 \mu \mathrm{l}$ of an aqueous solution of plasmid DNA (ca. $100 \mathrm{ng} / \mu \mathrm{l}$ ) was added to $50 \mu \mathrm{l}$ of electrocompetent cells, the mixture was pipetted into a $2 \mathrm{~mm}$ electroporation cuvette. The electroporation was performed in an electroporation vessel (Gene pulser) at 1650 $\mathrm{V}$. Then the suspension was transferred into an Eppendorf tube and mixed with $1 \mathrm{ml}$ LB medium. After $1 \mathrm{~h}$ of incubation at $37^{\circ} \mathrm{C}$, cells were plated as described above.

\subsubsection{Protein chemistry methods \& techniques}

\subsubsection{Sonication}

Sonication is a simple method used for the disruption of cells by ultrasounds. The high frequency sound wave induces formation of cavitations in the solution, the collapse of which produces sheer forces able to break cells. The advantage of this method for cell disruption over French press, freezing and thawing or lysozyme digestion is comparatively low viscosity of the lysate due to the nucleic acid fragmentation.

Pulsed mode of operation was applied (output control 8,60\% duty cycle) and sonication was carried out on ice, in 3 steps of 5 min each, with 5 min intervals between steps, to avoid overheating of the sample. 


\subsubsection{SDS polyacrylamide gel electrophoresis (SDS PAGE)}

SDS polyacrylamide gel electrophoresis was performed at various stages of purification to verify samples' purity, identity of the eluted proteins in chromatography purification, progression of enzymatic digestion etc. Due to the small size of the expressed proteins, tricine gels were applied (Schagger and von Jagow, 1987). Tricine, used as the trailing ion instead of glycine, allows a resolution of small proteins at lower acrylamide concentrations than in glycine-SDS-PAGE systems. A resolution of proteins, especially in the range between 5 and $20 \mathrm{kDa}$, is thus achieved without the necessity to use urea.

The guanidinium $\mathrm{HCl}$-free protein samples were prepared by mixing $20 \mu \mathrm{l}$ of protein solution with $5 \mu \mathrm{l}$ of sample buffer (SB) followed by 3 min incubation at $100{ }^{\circ} \mathrm{C}$. Due to rapid precipitation of SDS in contact with guanidine, the samples to be examined by PAGE after Ni-NTA chromatography under denaturing conditions had to be prepared in a following fashion: $20 \mu$ of the protein solution in a denaturing buffer was diluted with $400 \mu \mathrm{l} 20 \%$ trichloroacetic acid (TCA). The sample was incubated for $5 \mathrm{~min}$ at room temperature followed by centrifugation for $5 \mathrm{~min}$ at $20000 \times \mathrm{g}$. Supernatant was discarded by suction, the precipitated protein pellet was washed once by vortexing with $400 \mu$ ethanol. After centrifugation and ethanol removal, the protein pellet was resuspended in $20 \mu \mathrm{l}$ of $2 x$ SB and the sample was boiled for $3 \mathrm{~min}$.

\subsubsection{Visualization of separated proteins}

For visualization of the protein bands, the gels were stained in a Coomassie-blue solution. Background was cleared by incubation of the gel in a destaining solution. Both processes were greatly accelerated by brief heating with microwaves of the gel submerged in an appropriate solution.

\subsubsection{Protein expression, refolding and purification}

The One Shot ${ }^{\circledR}$ BL21 Star ${ }^{\text {TM }}$ (DE3) chemically competent cells were transformed with the plasmid DNA according to the protocol described above. Growth media contained kanamycin (for pET 28a transformed cells) or ampicillin (for pQE transformed cells) as selective antibiotics. Use of ampicillin as a selective antibiotic requires special care because $\beta$-lactamase is made in substantial amounts and is secreted into the medium, where it can destroy all of the ampicillin. In addition, ampicillin is susceptible to hydrolysis under acidic media conditions created by bacterial metabolism. This means 
that a culture in which the cells carry an unstable plasmid will be growing under ampicillin selection only until enough $\beta$-lactamase has been secreted to destroy the ampicillin in the medium. From that point on, cells that lack the plasmid will not be killed and will begin to overgrow the culture. This point is reached when the culture is barely becoming turbid (about $10^{7}$ cells per milliliter) for a typical pBR322-based plasmid, growing in amedium containing $50 \mu \mathrm{g}$ ampicillin per $\mathrm{ml}$. The presence of 200 $\mu \mathrm{g}$ ampicillin per milliliter delays this point to a slightly higher cell density, but given the catalytic activity of $\beta$-lactamase, it would not be feasible to add enough ampicillin to the medium to keep the cells under selection all the way to saturation. The precultures used as starting cultures were therefore grown at $28^{\circ} \mathrm{C}$, to slow bacterial growth, and not longer than $12 \mathrm{~h}$.

The overnight cultures of $20 \mathrm{ml}$ were used as an inoculum for the 1 liter culture. Typically, the cells were induced at $\mathrm{OD}_{600}=0.8-0.9$ by addition of IPTG ( $1 \mathrm{mM}$ final concentration) and cells were grown for the next $3 \mathrm{~h}$ with vigorous shaking at $37^{\circ} \mathrm{C}$. The cells were harvested by centrifugation $\left(5500 \times \mathrm{G}, 20 \mathrm{~min}, 4^{\circ} \mathrm{C}\right)$, bacterial pellets were resuspended in buffer $A$ and left for solubilisation at room temperature overnight. The cell suspension was sonicated $3 \times 5$ minutes using a macrotip (output control 7.5, $50 \%$ ) and centrifuged at $60000 \times \mathrm{g}$ for $1 \mathrm{~h}$ at $20{ }^{\circ} \mathrm{C}$. The resulting supernatant was incubated with a Ni-NTA slurry (Qiagen), equilibrated previously with buffer $\mathrm{A}$ for $1 \mathrm{~h}$ at room temperature with gentle agitation. Next, the mixture was loaded onto an empty column and washed with buffer $A$ and $B$. The protein was eluted with buffer $\mathrm{C}$. The fractions containing the desirable protein were pooled, concentrated and dialysed against buffer $D$ for removal of reducing agents. Subsequently, refolding of the protein was performed. For this purpose, the protein sample was stepwise diluted in buffer $\mathrm{E}$ in a 1:50 volume proportion. The refolding mixture was left with stirring at $4{ }^{\circ} \mathrm{C}$. After 3 days, the mixture was concentrated and dialysed against a low salt phosphate buffer, and then was loaded onto a cation exchange column (MonoS). Bound proteins were fractionated with a linear $\mathrm{NaCl}$ gradient. Fractions containing the protein of interest were pooled and dialysed against thrombin or Factor Xa cleavage buffers. Factor Xa digestion was performed for $72 \mathrm{~h}$ at room temperature in the $50 \mathrm{mM}$ Tris buffer $\mathrm{pH} 8.0$, $150 \mathrm{mM} \mathrm{NaCl}, 4 \mathrm{mM} \mathrm{CaCl}_{2}$ using $1 \mathrm{U}$ of the activated protease (Novagen) per $1 \mathrm{mg}$ of the fusion protein. Thrombin cleavage was carried out in the same conditions; $2 U$ of thrombin (Sigma) was used per $1 \mathrm{mg}$ of protein. 
The proteins of interest were separated from the HisTag and protease by gel filtration on the Superdex 75 prep grade 26/60 XR column. The buffer used contained $140 \mathrm{mM} \mathrm{NaCl}, 2.7 \mathrm{mM} \mathrm{KCl}, 10 \mathrm{mM} \mathrm{Na}_{2} \mathrm{HPO}_{4}, 1.8 \mathrm{mM} \mathrm{KH}_{2} \mathrm{PO}_{4}, 0.05 \% \mathrm{NaN}_{3}, \mathrm{pH}$ 7.3.

\subsubsection{Determination of protein concentration}

The concentration of proteins in solution was estimated by means of the Bradford colorimetric assay. $5 \mu \mathrm{l}$ of the protein sample was added to $1 \mathrm{ml}(10 \mathrm{x}$ diluted stock) Bradford reagent (BioRad) into a plastic cuvette. After gentle mixing, $A_{595}$ was measured and converted to the protein concentration on the basis of a calibration curve prepared for known concentrations of BSA.

A precise protein concentration determination was performed spectrophotometrically. Absorption at $280 \mathrm{~nm}$ was measured and converted to a protein concentration on the basis of theoretical extinction coefficients. It has been shown that it is possible to estimate the molar extinction coefficient $E_{\lambda}(\operatorname{Prot})$ of a protein from knowledge of its amino acid composition (Gill and Hippel, 1989). From the molar

extinction coefficient of tyrosine, tryptophan and cystine (cysteine residues do not absorb appreciably at wavelenghts $>260 \mathrm{~nm}$, while cystine does) at a given wavelength $\lambda$ the extinction coefficient of a protein can be computed using the equation:

$E_{\lambda}($ Prot $)=N u m b(T y r) x E x t_{\lambda}($ Tyr $)+N u m b(\operatorname{Trp}) x E x t_{\lambda}(\operatorname{Trp})+N u m b($ cystine $) x E x t_{\lambda}($ cystine $)$

The absorbance (A, optical density) can be calculated using the following formula:

$A_{\lambda}($ Prot $)=E_{\lambda}($ Prot $) /$ Molecular weight.

\subsubsection{X-ray crystallography}

\subsubsection{1 protein crystallization}

The purity and homogeneity of the proteins subjected to crystallization (i.e. NBP-4(382), NBP-4(-1-92) and CBP-4) was evaluated using N-terminal sequencing and mass spectrometry. IGF-I was purchased from GroPep (Australia). The binary (NBP-4/IGF-I) and ternary (NBP-4(3-82)/CBP-4/IGF-I) complexes were prepared by mixing equimolar amounts of the respective components. The complexes were separated from any excess of either protein by gel filtration on the Superdex S75 prep grade 26/60XR column. The buffer used contained $5 \mathrm{mM}$ Tris, $\mathrm{pH} 8.0,50 \mathrm{mM} \mathrm{NaCl}$ and $0.01 \% \mathrm{NaN}_{3}$. 
Preliminary determination of the range of favorable protein concentration was performed using a Pre-Crystallization Test (PCT, Hampton Research). The search of crystallization conditions was performed using Hampton Research crystallization screens I, II and Index (Hampton Research), as well as the in-house library of factorials.

Initial screening of crystallization conditions for NBP-4(3-82)/IGF-I binary complex yielded 3 hits. Crystal Screen I solutions 42 (0.05 $\mathrm{M} \mathrm{KH}_{2} \mathrm{PO}_{4}, 20 \%$ PEG 8000) and 43 (30\% PEG 1500) contained a quasi-crystalline material. The Index screen solution 37 (25\% w/v PEG 1500) produced micro crystals. The latter condition was further optimized in terms of PEG and protein concentrations. The largest influence on crystal formation, however, had addition of the Tris buffer (even at concentrations as low as $12.5 \mathrm{mM}$ ) promoting growth of large crystals. Crystallization occurred in various conditions of pH (7.25-8.45), PEG (20-23\%) and Tris (12.5-100 mM) concentrations. Micro seeding had to be applied to induce crystal growth in most drops. Crystallization of the NBP-4(3-82)/IGF-I binary complex was carried out with the sitting drop vapor diffusion method. The X-ray quality crystals were obtained from 23\% PEG 1500, 25 $\mathrm{mM}$ Tris $\mathrm{pH} 7.8$. They appeared in several days and grew to a final size of ca. $0.4 \times 0.2$ $x 0.2 \mathrm{~mm}$. Figure 14 encloses photographs of the crystals.

Crystals of NBP-4(1-92)/IGF-I were grown from the Index solution 74 (0.2 M lithium sulfate monohydrate, $0.1 \mathrm{M}$ Bis-Tris $\mathrm{pH} 5.5$ and 25\% w/v PEG 3350). The optimization of the conditions was achieved by dilution of the original solution by the factor of $1 / 16,1 / 8$ and 1/4, varying the size of drops and the ratios of protein and precipitant. Large, up to $0.8 \mathrm{~mm}$, long plates or plate cluster crystals grew throughout the range of these conditions.

Crystallization of the NBP-4(3-82)/CBP-4/IGF-I ternary complex occurred in the Crystal Screen solution 49 and 50 (1.0 M lithium sulfate monohydrate, 15\% w/v PEG 8000 and $0.5 \mathrm{M}$ lithium sulfate monohydrate, $2 \% \mathrm{w} / \mathrm{v}$ PEG 8000 , respectively), and the in-house factorial 30 (0.1 M ammonium sulfate, $0.1 \mathrm{M}$ Hepes/ $\mathrm{NaOH}$ pH 7.3, 20\% PEG 4000). The crystals grown in solution 49, although flawless in appearance (Figure 14 $\mathrm{H}$ ), demonstrated poor diffraction properties. Further optimization (constant $1 \mathrm{M}$ lithium sulfate concentration, varying concentration of PEG 8000: $0.5,1,1.5,2 \%$ ) produced large crystals of totally different morphology, diffracting up to $3 \AA$ (Figure $14 \mathrm{E}, \mathrm{F}$ and $\mathrm{G})$. The crystals of the first batch appeared after 2-6 days. It took 1 year for the optimized ones to emerge from the solution. These crystals were subsequently used 
for heavy metal derivatization. Factorial 30 yielded crystals diffracting up to $2.1 \AA$, but the conditions for their growth could not be repeated.

\subsubsection{Data collection and molecular replacement}

Prior to plunge freezing, the crystals of the NBP-4(3-82)/IGF-I binary complex were soaked for ca. $30 \mathrm{~s}$ in a drop of a reservoir solution containing $15 \% \mathrm{v} / \mathrm{v}$ ethylene glycol as cryoprotectant. The crystals belonged to the space group $P 2{ }_{1} 2{ }_{1}{ }_{1}$ and contained one complex per an asymmetric unit.

A lower-resolution dataset (up to $2.1 \AA$ ) was collected from a plunge frozen crystal at a rotating anode laboratory source. The high-resolution dataset up to $1.6 \AA$ was collected on the MPG/GBF beamline BW6 at DESY, Hamburg, Germany. Collected data were integrated, scaled and merged by XDS and XSCALE programs (Kabsch, 1993). The structure was determined by molecular replacement using the Molrep program from the CCP4 suite (CCP4, 1994). The structure of the complex of IGF-I and a fragment of the N-terminal domain of IGFBP-5 (miniNBP-5, PDB entry 1H59) was used as a probe structure (Zeslawski et al., 2001). Rotation search in the Patterson space yielded one peak of a height $7.56 \sigma$ over the highest noise peak of $3.75 \sigma$. Translation search gave a $16.28 \sigma$ peak over the noise height of $9.49 \sigma$. The initial Rfactor of the model was 0.49 . At this stage the free atom model improvement was performed using Arp/wArp (Perrakis et al., 2001). As the next step an iterative model building was performed using Arp/wArp (Perrakis et al., 1999). The initial model consisted of 137 residues and was completed and revised manually using Xfit software (McRee, 1999). Arp/wArp was used to add solvent atoms (Lamzin et al., 1993).

The structure was finally refined by the Refmac5 program (CCP4, 1994). Final electron density maps were of high quality; there were however no interpretable densities for side chains of residues Glu11, Arg16, Leu42, Glu66 and Glu81 of NPB-4 and Lys27, Arg37, Arg50 of IGF-I. These side chains were removed from the model. The final $R$ crystallographic factor was 0.186 and $R_{\text {free }}$ 0.2582. Data collection and refinement statistics are summarized in Table 3.

Crystals of the ternary complex were obtained in two conditions. One form, obtained from $1 \mathrm{M}$ lithium sulfate and 1 \% PEG 8000, diffracted up to $2.9 \AA$ and belonged to the space group $\mathrm{R} 3_{2}$. The second crystal, grown from $0.1 \mathrm{M}$ ammonium sulfate, 20\% PEG 4000 and $0.1 \mathrm{M}$ Hepes/NaOH, with the space group C2, diffracted up to $2.1 \AA$. Molecular replacement trials using the NBP4/IGF-I model were unsuccess 
ful. Experimental phasing was then performed using $\mathrm{R} 3_{2}$ crystals; we did not succeed in derivatizing $\mathrm{C} 2$ crystals. Soaking crystals with $2 \mathrm{mM}$ potassium hexabromoplatinate (IV) or $2 \mathrm{mM}$ uranylacetate for $24 \mathrm{~h}$ created two isomorphous derivatives. Native and derivative datasets were collected at the BW6 beamline in DESY, Hamburg. Datasets

Table 3. Data collection and refinement statistics for the NBP-4(3-82)/IGF-I complex

\begin{tabular}{|c|c|}
\hline \multicolumn{2}{|c|}{ Data Collection } \\
\hline Space group & $P 2{ }_{1}{ }_{1} 2_{1}$ \\
\hline Cell constants $(\AA)$ & $a=34.47 b=54.28 c=74.55$ \\
\hline Resolution range $(\AA)$ & $37-1.6$ \\
\hline Wavelength $(\AA)$ & $1.542,1.05$ \\
\hline Observed reflections & 86002 \\
\hline Unique reflections & 17605 \\
\hline \multicolumn{2}{|l|}{ Whole range: } \\
\hline Completeness (\%) & 92.1 \\
\hline$R_{\text {merge }}$ & 4.4 \\
\hline $\mathrm{l} / \sigma(\mathrm{I})$ & 16.86 \\
\hline \multicolumn{2}{|l|}{ Last shell: } \\
\hline Resolution range $(\AA)$ & $1.6-1.7$ \\
\hline Completeness (\%) & 76.9 \\
\hline$R_{\text {merge }}$ & 17.8 \\
\hline $\mathrm{I} / \sigma(\mathrm{I})$ & 5.45 \\
\hline \multicolumn{2}{|c|}{ Refinement } \\
\hline No. of reflections & 17388 \\
\hline Resolution $(\AA)$ & $30-1.6$ \\
\hline R-factor (\%) & 18.65 \\
\hline $\mathrm{R}_{\text {free }}(\%)$ & 25.82 \\
\hline Average $B\left(\AA^{2}\right)$ & 24.51 \\
\hline R.m.s bond length $(\AA)$ & 0.017 \\
\hline R.m.s. angles $\left({ }^{\circ}\right)$ & 1.83 \\
\hline \multicolumn{2}{|c|}{ Content of Asymmetric Unit } \\
\hline No. of protein complexes & 1 \\
\hline No. of protein residues/atoms & $144 / 1061$ \\
\hline No. of solvent atoms & 157 \\
\hline
\end{tabular}


Table 4. Data collection and refinement statistics for the NBP-4(3-82)/CBP-4 /IGF-I complex

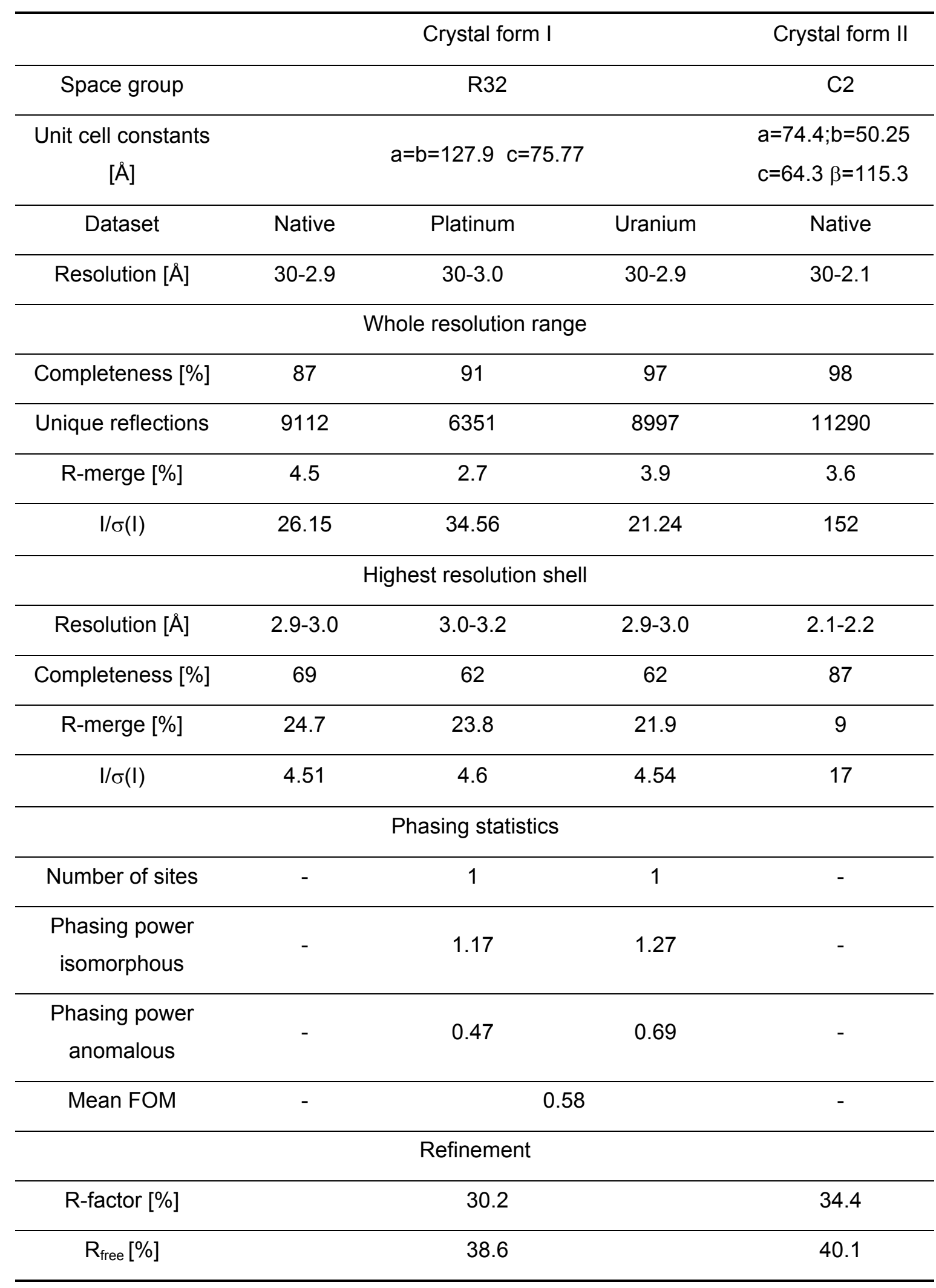


were indexed, integrated and scaled using a XDS software package. Heavy atom search and phase improvement were performed with the SHARP program (de La Fortelle and Bricogne, 1997) using both anomalous and isomorphous signals. The phases obtained were of good quality up to the resolution of $3.5 \AA$. The model of the NBP4/IGF-I complex was fitted to this map. All parts of the complex maintain the spatial organization found in the crystals of the NBP4/IGF-I binary complex. After placing the NBP-4/IGF part, polyalanine fragments were fitted into a broken electron density associated with the C-terminal domain. This model of the complex was later used as a molecular replacement probe for the C2 crystal data. The replacement solution was clear, however further model building and completion of the C-terminal part was not possible. The current R-factor of the ternary complex model is $34 \%$. Statistics of data collection, phasing and refinement are summarized in Table 4.

\subsubsection{NMR - experimental procedure}

All NMR experiments were carried out on a Bruker DRX 600 spectrometer equipped with a triple resonance, triple gradient $5 \mathrm{~mm}$ probehead, at $300 \mathrm{~K}$. The samples contained typically 0.1-0.5 mM protein in the PBS buffer supplemented with $10 \%{ }^{2} \mathrm{H}_{2} \mathrm{O}$. All the $1 \mathrm{D}{ }^{1} \mathrm{H}$ NMR spectra were recorded with a time domain of $32 \mathrm{~K}$ complex points and a sweep-width of $10,000 \mathrm{~Hz}$. The $2 \mathrm{D}{ }^{1} \mathrm{H}-{ }^{15} \mathrm{~N}-\mathrm{HSQC}$ spectra were recorded with a time domain of $1 \mathrm{~K}$ complex data points with 128 complex increments with a sweep width of $8 \mathrm{kHz}$ in the ${ }^{1} \mathrm{H}$ dimension and $2 \mathrm{kHz}$ in the ${ }^{15} \mathrm{~N}$ dimension.

\subsubsection{Isothermal titration calorimetry}

All experiments were carried out according to references provided by the manufacturer. Proteins (IGFBPs, their separate domains and IGF-I) were used at 0.25$0.4 \mathrm{mM}$ in PBS (140 mM NaCl, $2.7 \mathrm{mM} \mathrm{KCl} 10 \mathrm{mM} \mathrm{Na}_{2} \mathrm{HPO}_{4}, 1.8 \mathrm{mM} \mathrm{KH}_{2} \mathrm{PO}_{4}, 0.05$ $\mathrm{NaN}_{3}, \mathrm{pH}$ 7.3) and titrated from a $300 \mu$ syringe into a sample chamber holding 1.43 $\mathrm{ml}$ of $0.025-0.04 \mathrm{mM}$ respective binding partner. All solutions were degassed prior to measurements. Heat generated by protein dilution was determined in separate experiments by injecting protein solution into PBS filled sample chamber. All data were corrected for the heat of protein dilution. The details of the experimental and injection parameters are described below.

Parameters of the experiments:

total number of injections: 58 
volume of a single injection $[\mu l]: \quad 5$

duration of an injection [sec]: 10

intervals between injections [sec]: $\quad 400$

filter period [sec]:

equilibrium cell temperature $\left[{ }^{\circ} \mathrm{C}\right]$ : 20

initial delay [sec]: $\quad 60$

reference power $[\mu \mathrm{Cal} / \mathrm{sec}]:$

stirring speed [RPM]: $\quad 270$

All data were corrected for the heat of protein dilution. Data were fitted using $\chi^{2}$ minimization on a model assuming a single set of sites to calculate the binding affinity $\mathrm{K}_{\mathrm{D}}$, except for titrations involving the NBP-4-IGF binary complex with CBP-4, where only a 2 binding site model could be applied. All steps of the data analysis were performed using ORIGIN (V5.0) software provided by the manufacturer.

\subsubsection{Fluorescence measurements}

Binding of NBP-4 and CBP-4 to IGF-I was monitored by changes in the intrinsic tryptophan 3 fluorescence of a mutant BP1-NBP-4 (residues 1-3 of the IGFBP-1 followed by residues 5-82 of IGFBP-4). Experiments were performed in a $1 \mathrm{ml}$ fluorimeter cuvette at $20{ }^{\circ} \mathrm{C}$ using Perkin Elmer LS 50 B luminance spectrophotometer with spectral bandwidth of $5 \mathrm{~nm}$ for excitation and emission. The proteins and protein complexes were diluted to $4 \mu \mathrm{M}$ in $1 \mathrm{ml}$ of a buffer containing $140 \mathrm{mM} \mathrm{NaCl}, 2.7 \mathrm{mM}$ $\mathrm{KCl} 10 \mathrm{~mm} \mathrm{Na} \mathrm{HPO}_{4}, 1.8 \mathrm{mM} \mathrm{KH}_{2} \mathrm{PO}_{4}(\mathrm{pH} 7.3), 0.05 \% \mathrm{NaN}_{3}$. All spectra were corrected for buffer fluorescence. Excitation wavelength was adjusted to $295 \mathrm{~nm}$ to minimize tyrosine fluorescence contribution, and emission was scanned over the range of $305-500 \mathrm{~nm}$.

\subsubsection{IGF-IR autophosphorylation inhibition assay}

Confluent monolayers of the NIH 3T3 cells stably expressing human IGF-IR in $3.5 \mathrm{~cm}$ dishes were starved in DMEM containing $0.5 \%$ dialysed fetal calf serum. After $48 \mathrm{~h}$, cells were incubated without any hormone or with $5 \times 10^{-9} \mathrm{M}$ IGF-I or $1 \times 10^{-8} \mathrm{M}$ IGF-II; each sample was preincubated with increasing concentrations of different IGF-binding proteins from 0 to $1 \times 10^{-6} \mathrm{M}$ at room temperature for $1 \mathrm{~h}$. After a 10 min stimulation at $37^{\circ} \mathrm{C}$, the medium was removed and cells were lysed with $250 \mathrm{ml}$ of a lysing buffer (20 
$\mathrm{mM}$ Hepes pH 7.5, $150 \mathrm{mM} \mathrm{NaCl}, 10 \%$ glycerol, 1\% NP-40, $1.5 \mathrm{mM} \mathrm{MgCl}, 1 \mathrm{mM}$ EGTA), $10 \mathrm{mM}$ sodium orthovanadate, and the protease inhibitor cocktail Complete (Roche) for $10 \mathrm{~min}$ on ice. Subsequently, the cells were scraped off the plate and the insoluble material was separated by centrifugation for $20 \mathrm{~min}$ at $4{ }^{\circ} \mathrm{C}$. The protein concentration of the supernatant was determined using the BCA kit from Pierce according to the manufacturer's instructions. Equal protein concentration was incubated with the SDS sample buffer $(63 \mathrm{mM}$ Tris- $\mathrm{HCl} \mathrm{pH} 6.8,3 \%$ SDS, $10 \%$ glycerol, $0.05 \%$ bromophenolblue, $100 \mathrm{mM}$ DTT), boiled for $5 \mathrm{~min}$ and loaded on a $7.5 \%$ SDS-polyacrylamide gel. After electrophoresis the proteins were transferred on a nitrocellulose membrane, which first was blocked for $1 \mathrm{~h}$ with the $3 \%$ BSA containing phosphatebuffered saline-Tween (PBST), then incubated overnight with $1 \mathrm{mg} / \mathrm{ml}$ monoclonal anti-phosphotyrosine antibody 3-365-10 (Boehringer Mannheim) in PBST that contained 3\% BSA. Unbound antibody was removed by extensive washing. The blot was then incubated with the 1:10 000 diluted anti-mouse IgG-specific antibody conjugated with horse radish peroxidase (Boehringer Mannheim). The immunoblot was developed using the ECL kit from Amersham. 


\section{Results}

This section of the thesis contains results of the work in a logical, although not always chronological order. First, methods of IGFBP protein expression and routes to their purification are described. The results of investigation on protein structures and folding by means of NMR, and the subsequent constructs' optimisation trials are then presented. Crystals and X-ray analysis of the binary (NBP-4(3-82)/IGF-I and NBP-4(192)/IGF-I) and ternary (NBP-4(3-82)/CBP-4/IGF-I) complexes are reported, followed by the results of isothermal titration calorimetry studies. This part is followed by results of the experiments loosely connected with the mainstream project, such as a potential inhibitor of the IGF-I-IGFBP-5 interaction.

\subsection{Cloning, expression and purification of IGFBP domains}

\subsubsection{Protein purification strategies}

The topic of the constructs design, choice of expression vectors, fusion tags and means of their removal is covered in a detail in sections 4.2.1 and 4.2.2, pp. 43-46. The list of the constructs generated in this work, with references to the primers used, is enclosed in Table 5.

A starting point in the design of a purification workflow is learning about properties of a protein of interest, such as its theoretical isoelectric point ( $\mathrm{pl}$ ), molecular mass (MW) and extinction coefficient $\left(E_{280}\right)$. The information can be deduced solely on the basis of the amino acid composition with the aid of an Internet engine, ProtParam on www.expasy.ch for instance. Examination of already existing methods of purification of similar or homologous proteins can yield important information. Closer examination of the proteins' amino acid sequence may reveal potential cleavage/degradation sites. For example, proline often precedes basic residues at thrombin cleavage site (Mann et al., 1982).

Table 6 contains information on the basic physicochemical properties of the proteins produced or used in the work. The theoretical pl informs of the net charge of a protein placed in a solvent of a given $\mathrm{pH}$. Thus a proper buffer and ion-exchanger (cation- or anion-exchanger) medium combination can be chosen. It is common, how- 
Table 5. List of constructs produced in the work

\begin{tabular}{|c|c|c|c|c|c|}
\hline № & Name (residues) & $\begin{array}{l}\text { Vector and restriction } \\
\text { sites }\end{array}$ & Tag & $\begin{array}{l}\text { Cleavage } \\
\text { site }\end{array}$ & $\begin{array}{l}\text { Primers } \\
\text { used }\end{array}$ \\
\hline 1.1 & CBP-3 C-His (G184-K264) & pET-28a Nco I/Xho I & C-His & - & 1,3 \\
\hline 1.2 & CBP-3 QE (G184-K264) & pQE-80 BamH I/Hind III & $\mathrm{N}$-His & TagZyme & 2,4 \\
\hline 2.1 & IGFBP-4 FL (D1-E237) & BP4-2/pFDX500 (ROCHE) & - & - & - \\
\hline 2.2 & IGFBP-4 FL (D1-A232) & pET-28a BamH I/Xho I & $\mathrm{N}-\mathrm{His} / \mathrm{T7}$ & Thrombin & 9,16 \\
\hline 3.1 & miniNBP-4 Xa (A39-L82) & pET-28a BamH I/Hind III & N-His/T7 & Factor Xa & 5,7 \\
\hline 3.2 & miniNBP-4 Thr (A39-L82) & pET-28a BamH I/Hind III & $\mathrm{N}-\mathrm{His} / \mathrm{T7}$ & Thrombin & 6,7 \\
\hline 4 & NBP-4 (A3-L82) & pET-28a BamH I/Hind III & $\mathrm{N}-\mathrm{His} / \mathrm{T7}$ & Factor Xa & 7,8 \\
\hline 5 & NBP-4 (D1-L92) & pET-28a BamH I/Xho I & $\mathrm{N}-\mathrm{His} / \mathrm{T7}$ & Factor $\mathrm{Xa}$ & 9,13 \\
\hline 6 & DE-NBP-4 (D1-L82) & pET-28a BamH I/Hind III & $\mathrm{N}-\mathrm{His} / \mathrm{T7}$ & Factor Xa & 7,9 \\
\hline 7 & APW-NBP-4 (APWH5-L82) & pET-28a BamH I/Hind III & $\mathrm{N}-\mathrm{His} / \mathrm{T} 7$ & Factor $\mathrm{Xa}$ & 7,10 \\
\hline 8 & BP3-NBP4 (G1-V11, H5-L82) & pET-28a BamH I/Hind III & $\mathrm{N}-\mathrm{His} / \mathrm{T} 7$ & Factor $\mathrm{Xa}$ & $7,11,12$ \\
\hline 9.1 & CBP-4 Thr (G151-A232) & pET-28a BamH I/Xho I & $\mathrm{N}-\mathrm{His} / \mathrm{T7}$ & Thrombin & 14,16 \\
\hline 9.2 & CBP-4 Xa (G151-A232) & pET-28a BamH I/Xho I & $\mathrm{N}-\mathrm{His} / \mathrm{T7}$ & Factor Xa & 15,16 \\
\hline 10 & CBP-4 d168-174 (G151-A232) & pET-28a BamH I/Xho I & $\mathrm{N}-\mathrm{His} / \mathrm{T7}$ & Fhrombin & 34,35 \\
\hline 11 & CBP-4 d167-177 (G151-A232) & pET-28a BamH I/Xho I & $\mathrm{N}-\mathrm{His} / \mathrm{T7}$ & Thrombin & 36,37 \\
\hline 12 & CBP-4 d216-223 (G151-A232) & pET-28a BamH I/Xho I & $\mathrm{N}-\mathrm{His} / \mathrm{T7}$ & Thrombin & 38,39 \\
\hline 13 & CBP-4 d213-225 (G151-A232) & pET-28a BamH I/Xho I & $\mathrm{N}-\mathrm{His} / \mathrm{T7}$ & Thrombin & 40,41 \\
\hline 14 & CBP-4 W (G151-W206) & pET-28a BamH I/Xho I & $\mathrm{N}-\mathrm{His} / \mathrm{T7}$ & Thrombin & 14,42 \\
\hline 15 & IGFBP-5 FL (L1-V252) & BP5-3/pFDX500 (ROCHE) & - & - & - \\
\hline 16 & $\operatorname{miniNBP-5}(\mathrm{A} 40-192)$ & pQE-30 BamH I/Hind III & $\mathrm{N}-\mathrm{His}$ & Thrombin & - \\
\hline 17 & miniNBP-5 L61Y (A40-192) & pET-28a BamH I/Hind III & N-His/T7 & Thrombin & 30,31 \\
\hline 18 & miniNBP-5 L74M (A40-192) & pET-28a BamH I/Hind III & $\mathrm{N}-\mathrm{His} / \mathrm{T7}$ & Thrombin & 32,33 \\
\hline 19.1 & CBP-5 T (D135-D247) & pET-28a BamH I/Hind III & $\mathrm{N}-\mathrm{His} / \mathrm{T7}$ & Thrombin & 17,18 \\
\hline 19.2 & CBP-5 TZ (D135-D247) & pQE-80 BamH I/Hind III & $\mathrm{N}-\mathrm{His}$ & TagZyme & Subcl. \\
\hline 20.1 & CBP-5 T (G170-D247) & pET-28a BamH I/Hind III & $\mathrm{N}-\mathrm{His} / \mathrm{T7}$ & Thrombin & 18,19 \\
\hline 20.2 & CBP-5 X (G170-D247) & pET-28a BamH I/Hind III & $\mathrm{N}-\mathrm{His} / \mathrm{T7}$ & Factor Xa & 18,20 \\
\hline 20.3 & CBP-5 TZX (G170-D247) & pQE-80 BamH I/Hind III & $\mathrm{N}-\mathrm{His}$ & TagZyme, Xa & Subcl. \\
\hline 21 & IGFBP-6 FL (A1-G213) & pET-28a BamH I/Xho I & $\mathrm{N}-\mathrm{His} / \mathrm{T7}$ & Thrombin & 21,25 \\
\hline 22 & NBP-6 (A1-A82) & pET-28a BamH I/Xho I & $\mathrm{N}-\mathrm{His} / \mathrm{T7}$ & Thrombin & 21,22 \\
\hline 23 & NBP-6 (A1-S96) & pET-28a BamH I/Xho I & $\mathrm{N}-\mathrm{His} / \mathrm{T7}$ & Thrombin & 21,23 \\
\hline 24.1 & CBP-6 (G137-G213) & pET-28a BamH I/Xho I & $\mathrm{N}-\mathrm{His} / \mathrm{T7}$ & Thrombin & 24,25 \\
\hline 24.2 & CBP-6 NoTag(G137-G213) & pET-28a Nco I/Xho I & - & - & 25,27 \\
\hline 24.3 & CBP-6 C-His (G137-G213) & pET-28a Nco I/Hind III & C-His & - & 26,27 \\
\hline 25 & NBP-5 (L1-E83) & pET-28a BamH I/Hind III & $\mathrm{N}-\mathrm{His} / \mathrm{T7}$ & Factor Xa & 44,45 \\
\hline 26 & NBP-4 (G5-L82) & pET-28a BamH I/Hind III & $\mathrm{N}-\mathrm{His} / \mathrm{T7}$ & Factor Xa & 46,7 \\
\hline 27 & ConsBP3-NBP-4 (H5-L82) & pET-28a BamH I/Hind III & $\mathrm{N}-\mathrm{His} / \mathrm{T7}$ & Factor Xa & $47,48,7$ \\
\hline
\end{tabular}

Constructs equivalent on the protein level are numbered as X.1, X.2 etc. 
ever, that a protein of an acidic $\mathrm{pl}$ would bind to a cation-exchanger resin at $\mathrm{pH}>\mathrm{pl}$. This can be observed for the N-terminal domains of IGFBP-4 and -5 , which can bind MonoS medium in a buffer of $\mathrm{pH} 7.3$ even though their theoretical isoelectric points are low (6.3 and 5.28, respectively). Polar arrangement of the charged residues, resulting in positively charged patches, can account for this phenomenon. Masses of all proteins discussed in this work fall between fractionation ranges of the Superdex 75 resin, i.e. 3000-60 $000 \mathrm{Da}$. A known extinction coefficient of a protein enables quick estimation of the yield on the basis of the elution profile (height and area of the peak) monitored at a given wavelength.

Table 6. Biophysical properties of the investigated proteins

\begin{tabular}{llccc}
\hline oo & Name & MW $(\mathrm{Da})^{*}$ & $\mathrm{E}_{280}\left(\mathrm{M}^{-1} \mathrm{~cm}^{-1}\right)^{\star *}$ & $\mathrm{pl}$ \\
\hline- & IGF-I & 7649 & 4200 & 7.76 \\
$2.1,2.2$ & IGFBP-4 (fl) & 25954 & 10730 & 6.87 \\
15 & IGFBP-5 (fl) & 28555 & 15730 & 8.59 \\
$3.1,3.2$ & miniNBP-4 & 4681 & 2800 & 8.53 \\
16 & miniNBP-5 & 6095 & 2800 & 7.88 \\
4 & NBP-4 (3-82) & 8475 & 3280 & 7.65 \\
5 & NBP-4 (1-92) & 9803 & 3280 & 5.28 \\
6 & NBP-4 (1-82) & 8719 & 3280 & 6.30 \\
7 & APW-NBP-4(5-82) & 8645 & 8970 & 7.65 \\
8 & BP3-NBP-4(5-82) & 9173 & 3280 & 7.62 \\
$9.1,9.2$ & CBP-4 & 9096 & 7330 & 7.81 \\
25 & NBP-5(1-83) & 8868 & 2000 & 5.28 \\
$19.1,19.2$ & CBP-5 (IEGR etension) & 9578 & 11170 & 9.57 \\
- & His/T7-Tag & 4171 & - & 12.3 \\
- & PQE-HisTag & 1872 & - & 9.45 \\
\hline
\end{tabular}

${ }^{*}$ Mol. weight is calculated for the proteins with oxidized cysteines.

${ }^{* *} E($ Prot $)=N u m b(T y r) \times \operatorname{Ext}($ Tyr $)+N u m b(T r p) \times \operatorname{Ext}($ Trp $)+N u m b($ Cystine $) \times \operatorname{Ext}($ Cystine $)$

Purification of all IGFBPs constructs followed similar protocol, involving three different liquid chromatography steps: immobilized metal affinity chromatography on Ni-NTA, cation exchange followed by gel filtration chromatography. The major variations of the procedure considered the shape and length of $\mathrm{NaCl}$ gradients at the cation-exchange chromatography step (related to the strength of protein-column interaction, which depends on pl) and conditions of the His- or His/T7-tag cleavage 
(determined by the use of different enzymes). Figure 7 shows a flow-scheme of purification steps. Below follows a short description of the steps, and rationales behind some actions is explained. Tips and hints facilitating the work with the proteins are presented for anyone interested in continuing the project.

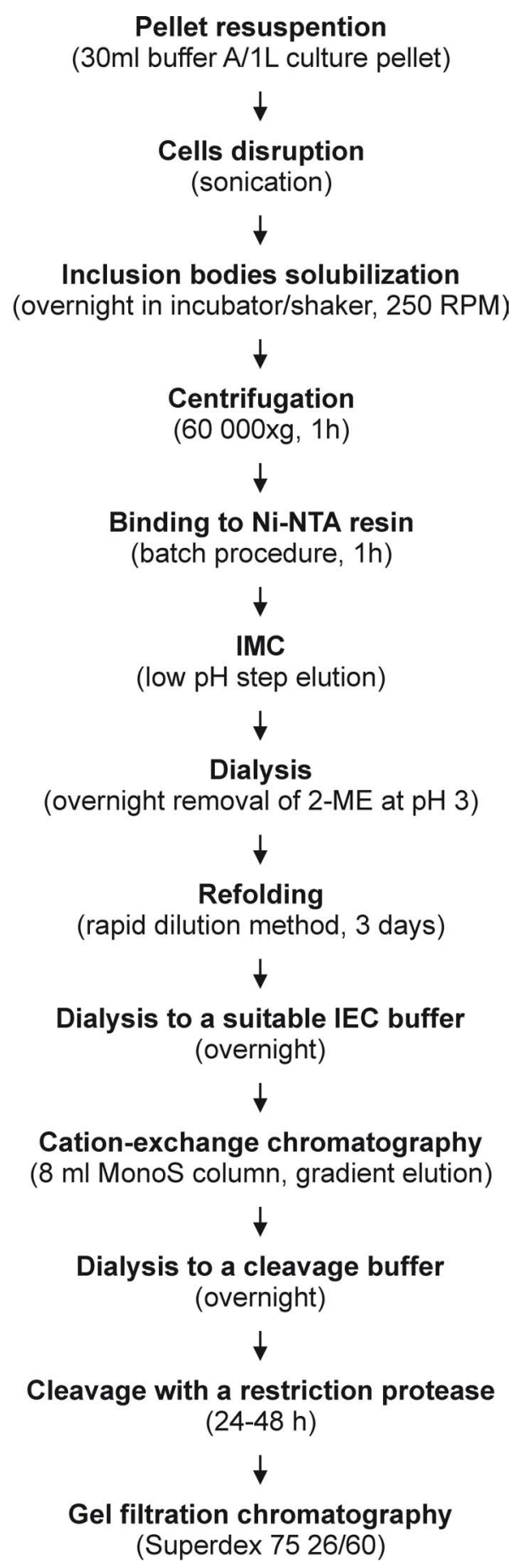

Figure 7. Purification of IGFBP constructs. 


\subsubsection{Solubilization of inclusion bodies}

The heterologous expression of foreign genes in $E$. coli often leads to production of overexpressed proteins in insoluble inclusion bodies (IBs). This is a case for all the proteins discussed here; therefore the IBs solubilization and refolding of the proteins into native states were carried out. Solubilization must result in a monomolecular dispersion and the minimum non-native intra- or inter-chain interactions. Solubilization was achieved by a ca. $12 \mathrm{~h}$ incubation, accompanied by vigorous stirring of previously disrupted cells in solutions containing $6 \mathrm{M}$ guanidinium hydrochloride, under strong reducing conditions (20 $\mathrm{mM} \beta-\mathrm{ME})$. Any insolubilities were removed and samples were clarified by centrifugation.

\subsubsection{Immobilized metal affinity chromatography (IMAC)}

Presence of the 6-His-Tag in all the expressed proteins enabled the use of nickel affinity chromatography under reducing conditions. The $\mathrm{pH}$ of a supernatant prepared in as described above was adjusted to 8.0 with $1 \mathrm{M} \mathrm{NaOH}$ (as optimal for binding to the Ni-NTA resin). A Ni-NTA slurry was added and binding was performed for $1 \mathrm{~h}$ with agitation. A 250-ml notched flask proved to be most adequate for this task. The ratio of the Ni-NTA matrix used to the amount of the His-tagged protein is crucial for purity of the protein. Below are two examples of purifications where too large or proper amounts of the resin were used. Two techniques of elution are also presented. Figure 8 shows that the bulk of impurities can be separated from the protein of interest using a $\mathrm{pH}$ gradient, a downside of this approach being severe dilution of the protein (Figure 8, left panel). It is more reasonable to use less resin and perform a stepwise elution, obtaining a pure, concentrated protein in a much shorter time (Figure 8, right).

\subsubsection{Refolding}

After an initial IMAC purification performed under denaturing conditions, the fractions containing target proteins were pooled, excess of $\beta$-ME was added, and the pool was dialyzed against $6 \mathrm{M} \mathrm{GuaHCl}$ of $\mathrm{pH}$ 3.0. The goal of this step was to reduce all disulfide bridges and then remove the reducing reagnt without causing reoxidation of cysteines. Recreation of disulfide bridges cannot occur at low $\mathrm{pH}$ due to the $\mathrm{SH}$ protonation.

Refolding is initiated by reducing the concentration of a denaturant. This can be performed by one- or multi-step dialyses, buffer exchange by gel filtration, applying a gradient of a refolding buffer when, the protein of interest is immobilized on the IMAC 


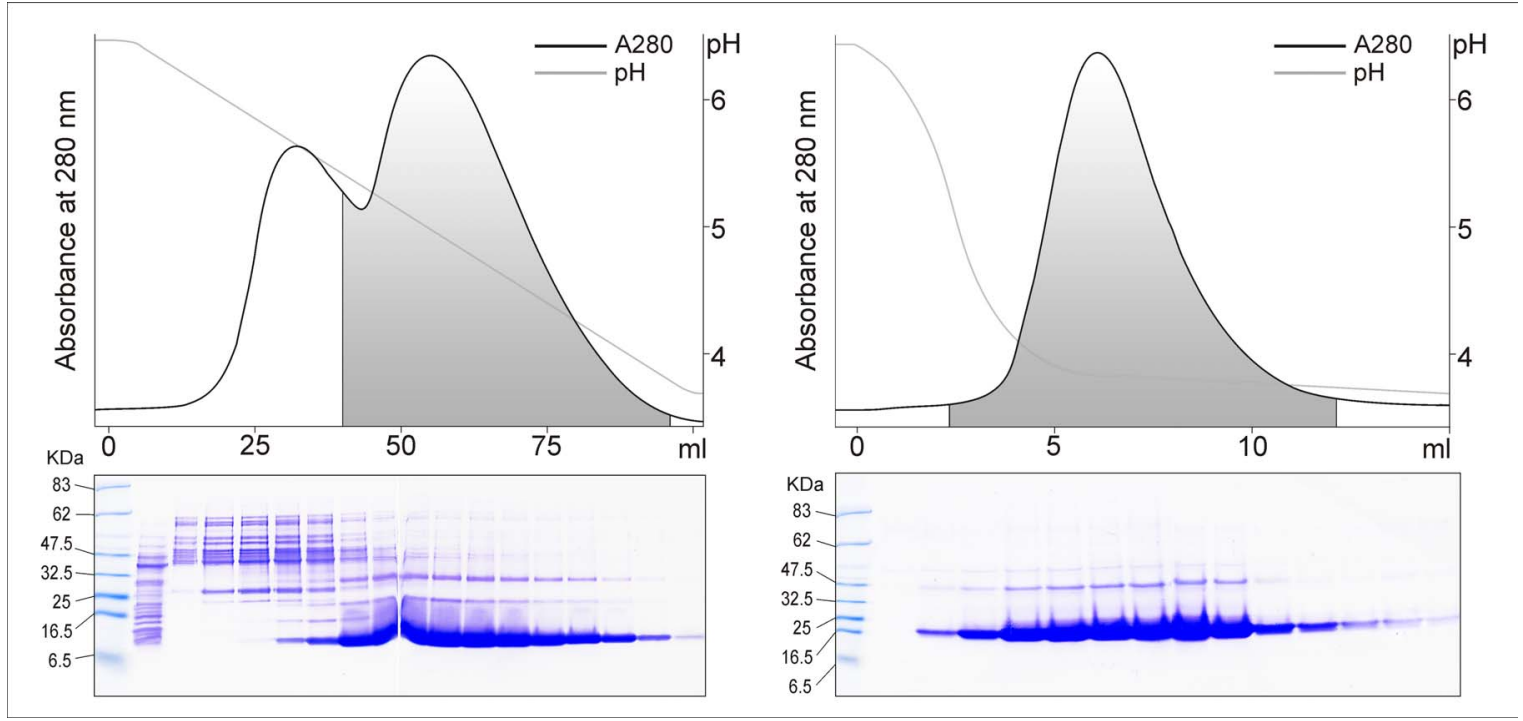

Figure 8. Comparison of two approaches for protein purification on the Ni-NTA resin. Left panel: a $100 \mathrm{ml} \mathrm{pH}$ gradient $(\mathrm{pH} 6.5-\mathrm{pH} 3.5)$ at $1 \mathrm{ml} / \mathrm{min}$ was used to elute His/T7-tagged NBP4(3-82) bound to $9 \mathrm{ml}$ of the Ni-NTA resin; $1.5 \mathrm{ml}$ fractions were collected. Every third fraction was examined by PAGE: line 1, marker: line 2, unbound proteins, lines 3-19, fractions 4-47. Right panel: His/T7-CBP-4 was eluted from $3 \mathrm{ml}$ of the Ni-NTA matrix in a single step at 0.5 $\mathrm{ml} / \mathrm{min}$. Fractions of $1 \mathrm{ml}$ were collected and analyzed by PAGE (lines 2-14). Pools are indicated as gray areas.

matrix (solid phase refolding), or by dilution (reviewed by Tsumoto et al., 2003). For refolding of the IGFBP constructs, a rapid (pulse) dilution technique was applied. The volume of the refolding buffer was adjusted to the total amount of protein to maintain its final concentration at a range of $0.1-0.2 \mathrm{mg} / \mathrm{ml}$. The volume ratio of the denaturant containing sample to the buffer was kept at 1:50, resulting in the final Gua- $\mathrm{HCl}$ concentration of less than $0.15 \mathrm{M}$. Dilution of the the protein containing solution was accomplished by injecting 100-200 $\mu \mathrm{l}$ aliquots into a refolding solvent, and refolding was allowed to occur for a period of 10-20 min before addition of the next aliquot. This way a high concentration of folding intermediates was avoided, which could otherwise lead to aggregation.

Dilution into a buffer means a collapse into a rigid structure, which cannot fluctuate or convert to the native structure, without a presence of low concentrations of denaturant or other low molecular co-solutes, often referred to as 'molecular chaperones". Arginine- $\mathrm{HCl}$ and glycerol were therefore present as aggregation suppressors, and GSH-GSSG redox system was supplied to enable shuffling of the disulfide bridges. Glutathions exert their function by cycles of covalent binding to the free thiol groups and their release with assistance of an atmospheric oxygen, pro- 
ducing GSSG, H2O and Cys-Cys bridges. This disulfide shuffling mimics the action of disulfide isomerase enzyme in vivo, preventing the structure from being prematurely rigidified by forming disulfide linkages. Sufficient time is thus given for the protein to collapse into a global free energy minimum state. The refolding was conducted for 3 days with vigorous steering and free access of air. The recovery of the protein of interest varied between $70-95 \%$. In general, the C-terminal constructs of IGFBPs were more susceptible to aggregation than $\mathrm{N}$-terminal ones.

\subsection{5 lon exchange chromatography}

A high-resolution chromatographic medium, MonoS, was used at a second purification step. Monodispersive, 5- $\mu \mathrm{m}$ bed support particles and a large protein binding capacity of the 8-ml column, ranging up to $20 \mathrm{mg} / \mathrm{ml}$, assured excellent resolution of absorbed proteins. Bound proteins were fractionated with a linear $\mathrm{NaCl}$ gradient, the length of which was chosen individually for each construct on the basis of pilot experiments (see Figure 9 for examples).

\subsubsection{Gel filtration chromatography}

This final step of purification enabled separation of the proteins from digested tag peptides, and the restriction protease used for tag cleavage. Complete exchange of the buffer was also possible, removing any low molecular weight substances, which could interfere with NMR measurements (such as Tris, EDTA, protease inhibitors, glycerol etc.) The column used, a $320-\mathrm{ml}$ Superdex 75 prep grade, is characterized by good resolution in the range 3-60 kDa. To make the best of its capability, low flow rates $(0.75-1.25 \mathrm{ml} / \mathrm{min})$ were used and samples not larger than $6 \mathrm{ml}$ were loaded on the column.

\subsection{Preliminary functional and structural studies}

\subsubsection{Gel filtration mobility shift assay}

To qualitatively assess IGF-I binding activity of the purified proteins, an analytical-scale gel filtration binding assay was carried out. The proteins were mixed in approximately equimolar ratios and separated on an analytical 23-ml Superdex 75 column. Different 
combinations of the proteins were examined to cover all possible interactions. Figure 10 summarizes the results of an assay performed for miniNBP-5, CBP-5 and IGF-I.
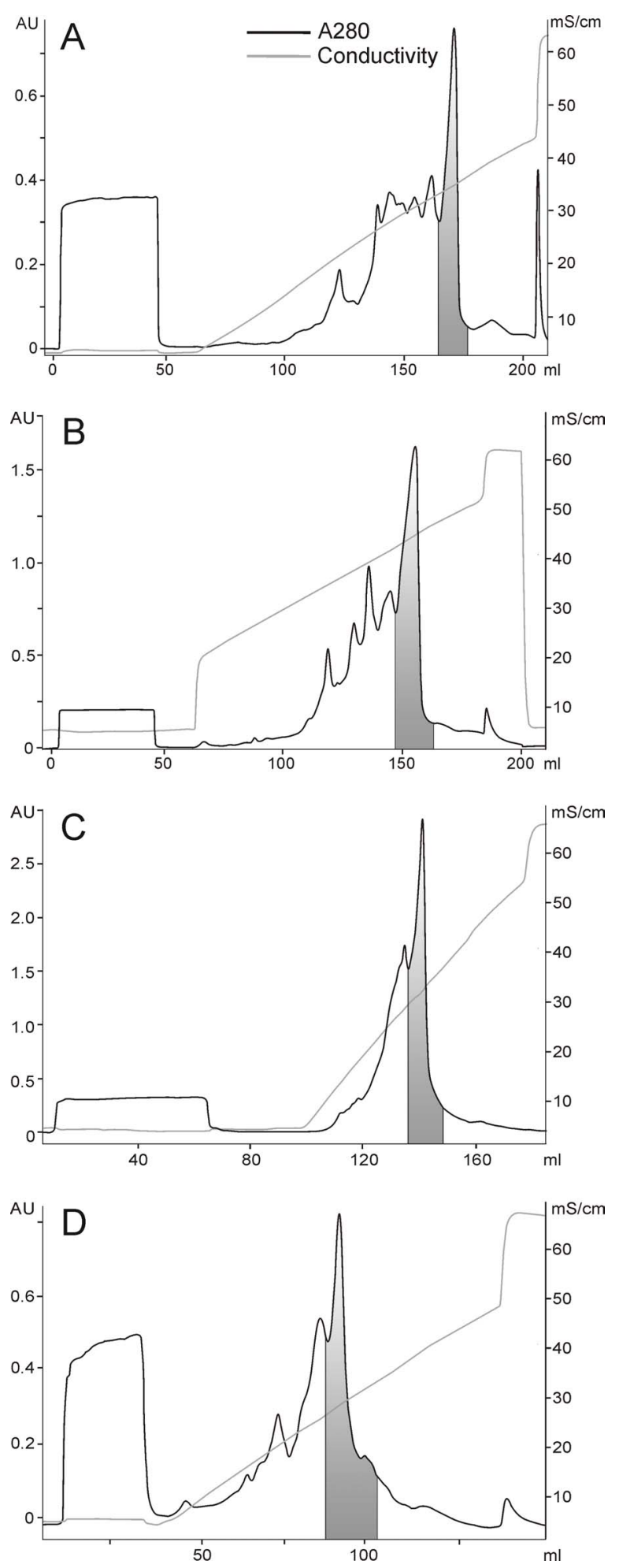

Figure 9. Four examples of separations of various IGFBP constructs by chromatography on a 8-ml cation exchange column. A: purification of CBP-4; bound proteins were fractionated with a $10-700 \mathrm{mM} \mathrm{NaCl}$ gradient in 17.5 column volumes (CV); B: CBP-5 purification: the protein was eluted with a 15 CV-long, 250-800 $\mathrm{mM} \mathrm{NaCl}$ gradient; C: NBP-4(3-82) was eluted with a 20-800 mM $\mathrm{NaCl}$ gradient in $5 \mathrm{CV}$; D: NBP-4(1-92) was fractionated with a $15 \mathrm{CV}, 10-700 \mathrm{mM} \mathrm{NaCl}$ gradient. Pooled fractions are indicated as gray areas. 
Similar elution profiles were obtained for combinations of CBP-4, IGF-I and miniNBP-4 or NBP-4(3-82). The experiment proved that all tested N-terminal constructs formed binary complexes with IGF-I, while C-terminal domains required the presence of amino-terminal domains for ternary complexes formation with IGF-I. Formation of hybrid ternary complexes (i.e. NBP-5/CBP-4/IGF-I and NBP-4/CBP5/IGF-I) was also observed.

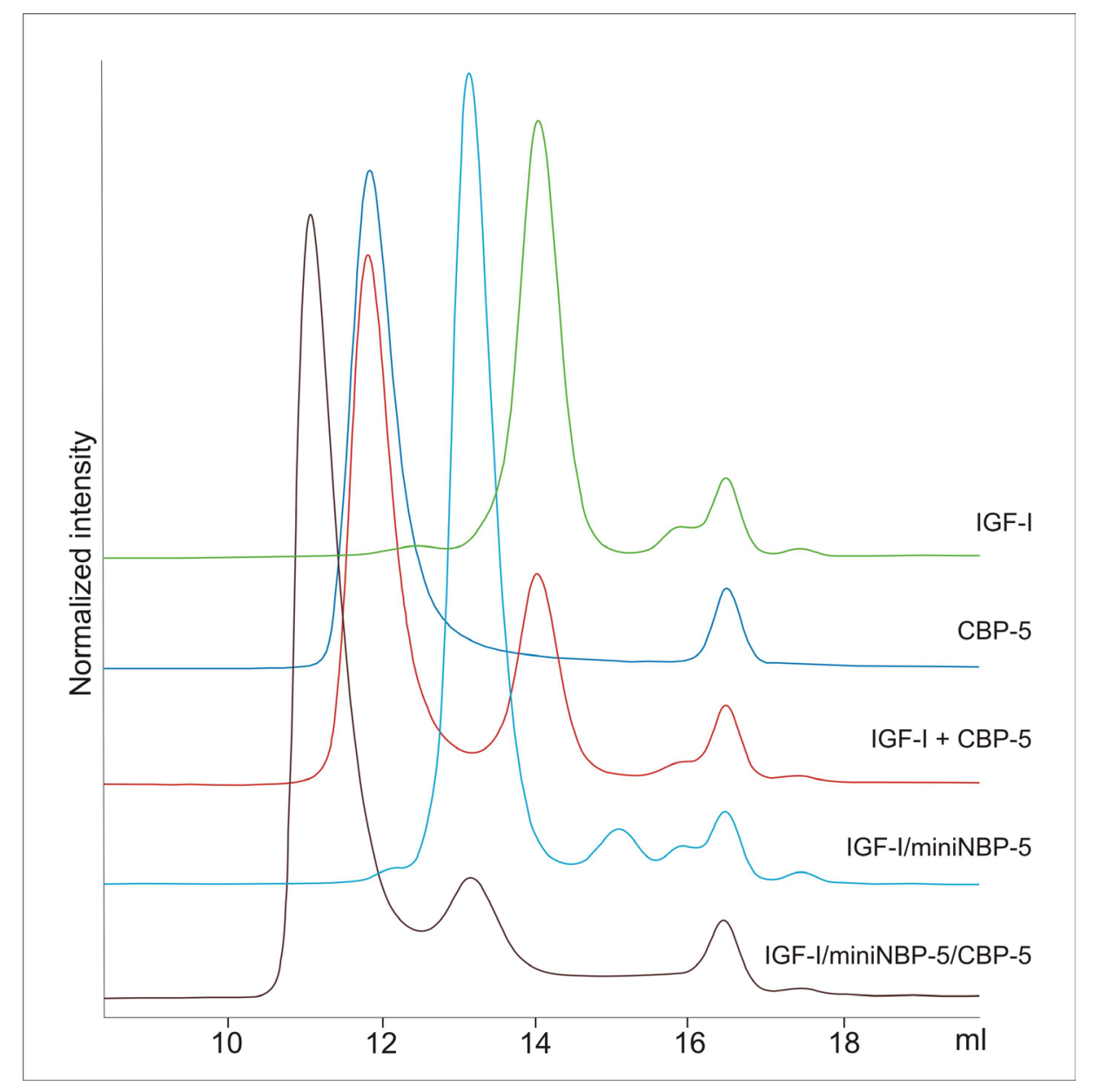

Figure 10. The gel filtration binding assay for miniNBP-5, CBP-5 and IGF-I. The proteins were mixed in approximately equal molar ratios in the indicated combinations, and separated on the analytical Superdex $7510 / 30$ column. Elution profiles are superimposed to visualize peak shifts.

To observe the formation of a stable protein complex in a gel filtration experiment, binding must occur with at least medium-low micromolar affinity. Higher $K_{D}$ values cause the complex to dissociate on the column. Possible weak interactions between the C-terminal domains and IGF-I or C-domains and N-terminal constructs could not be 
therefore ruled out on the basis of this experiment. NMR, a technique able to detect even very weak interactions (i.e. in the millimolar range), was therefore used to address the problem of inter-domain and C-domain-IGF-I interaction.

\subsubsection{NMR studies on the folding and domain organization of IGFBPs}

Figure 11 shows one-dimensional ${ }^{1} \mathrm{H}$ NMR spectra of the full length IGFBP-3, -4 and 5 and isolated N- and C-domains of IGFBP-4 and -5. Inspection of such spectra yields semi-quantitative information on the extent of folding in partially structured proteins or their domains (Rehm et al, 2002). The spectra of full-length IGFBP-3, -4, and -5 exhibit a substantial peak at $8.3 \mathrm{ppm}$ and some signals downfield (that is shifted to higher ppm values) close to the noise level. The appearance of intensities at chemical shifts near $\sim 8.3 \mathrm{ppm}$ is an indicator for a disordered protein, as this is a region characteristic of backbone amides in random coil configuration (Wüthrich, 1986). On the other hand large signal dispersion beyond $8.3 \mathrm{ppm}$ proves a protein to be folded. Due to the different chemical environment and thus the varying shielding effects the resonances of single protons will be distributed over a wide range of frequencies. A typical intensity pattern of a folded protein can be observed in spectra of the $\mathrm{N}$-terminal domain of IGFBP-4 (residues 1-92) and IGFBP-5 (residues 1-83). The spectra of the C-terminal fragments, CBP-4(151-232) and CBP-5(170-247) indicate that there are some unstructured regions located also in the C-terminal fragments. Based on these spectra it can be concluded that the whole central variable domain of IGFBP-4 and -5 is in a random coil conformation. The amide region of a 1D spectrum of the full length IGFBP3 obtained from human cell cultures is also shown to compare its extent of folding to that of the artificially refolded proteins. Thus, structure-wise, the properties of IGFBPs are the same whether expressed in E. coli or in the mammalian expression system.

\subsubsection{Optimization of the carboxyl-terminal construct of IGFBP-4}

1D NMR experiments revealed that the $\mathrm{COOH}$-terminal fragments of IGFBP-4, and -5 contained disordered polypeptide fragments. Such flexible sections of proteins are known to inhibit crystallization or result in crystals of low quality, which in fact was observed for the ternary complex of IGFBP-4(3-82)/IGFBP-4(151-232)/IGF-I.

Assuming that disordered regions are most likely to occur within the longest stretches of amino acids between cysteines of disulfide bridges, four deletion mutants were designed. The deletions spanned different I engths of polypeptide chains and 


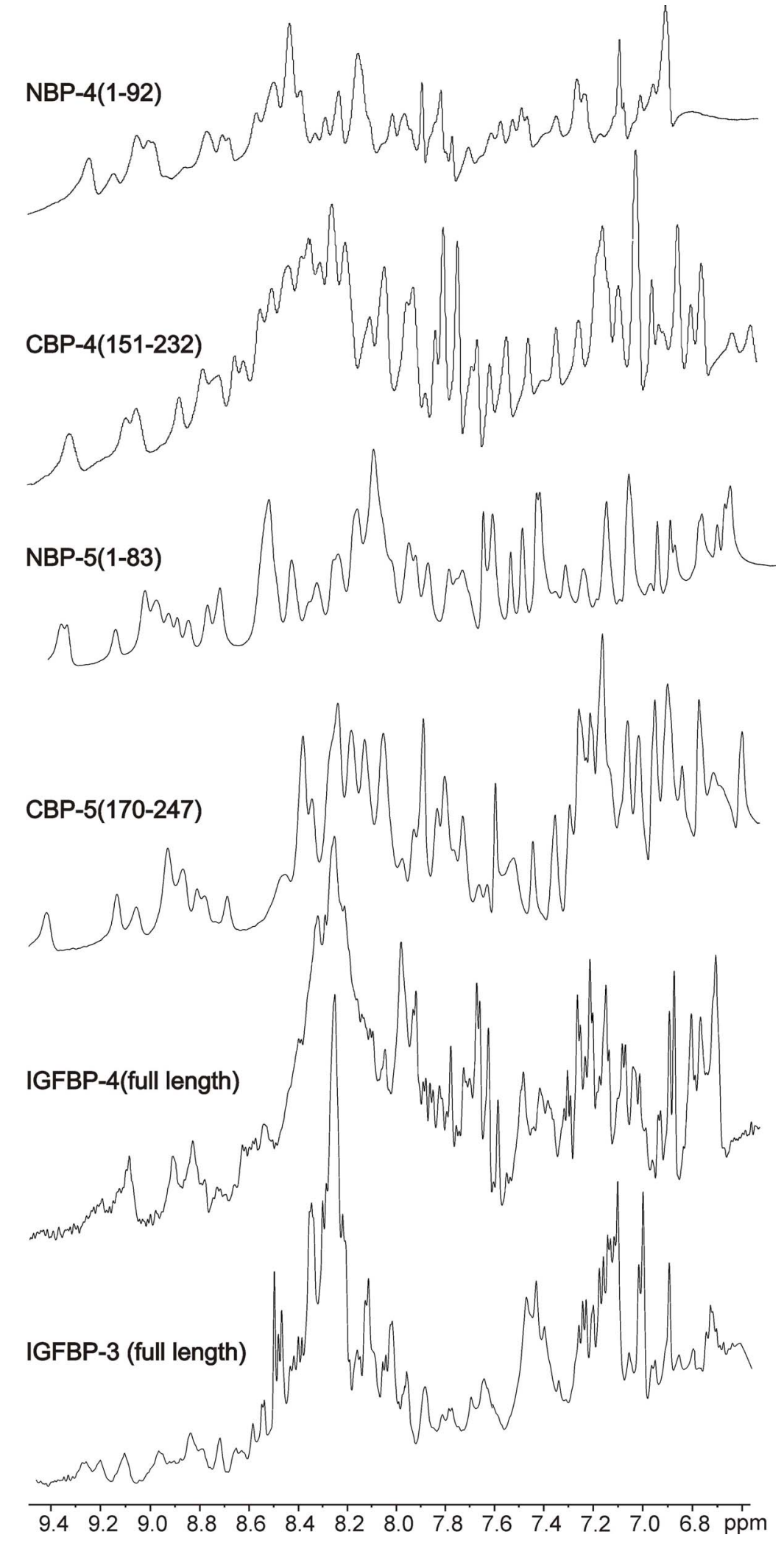

Figure 11. Characterization of IGFBP structures by one-dimensional NMR spectroscopy. Amide regions of ${ }^{1} \mathrm{H}$ NMR spectra of the full length IGFBP-3, -4 , and the isolated $\mathrm{N}$ - and $\mathrm{C}$ domains of IGFBP-4 and -5 . 

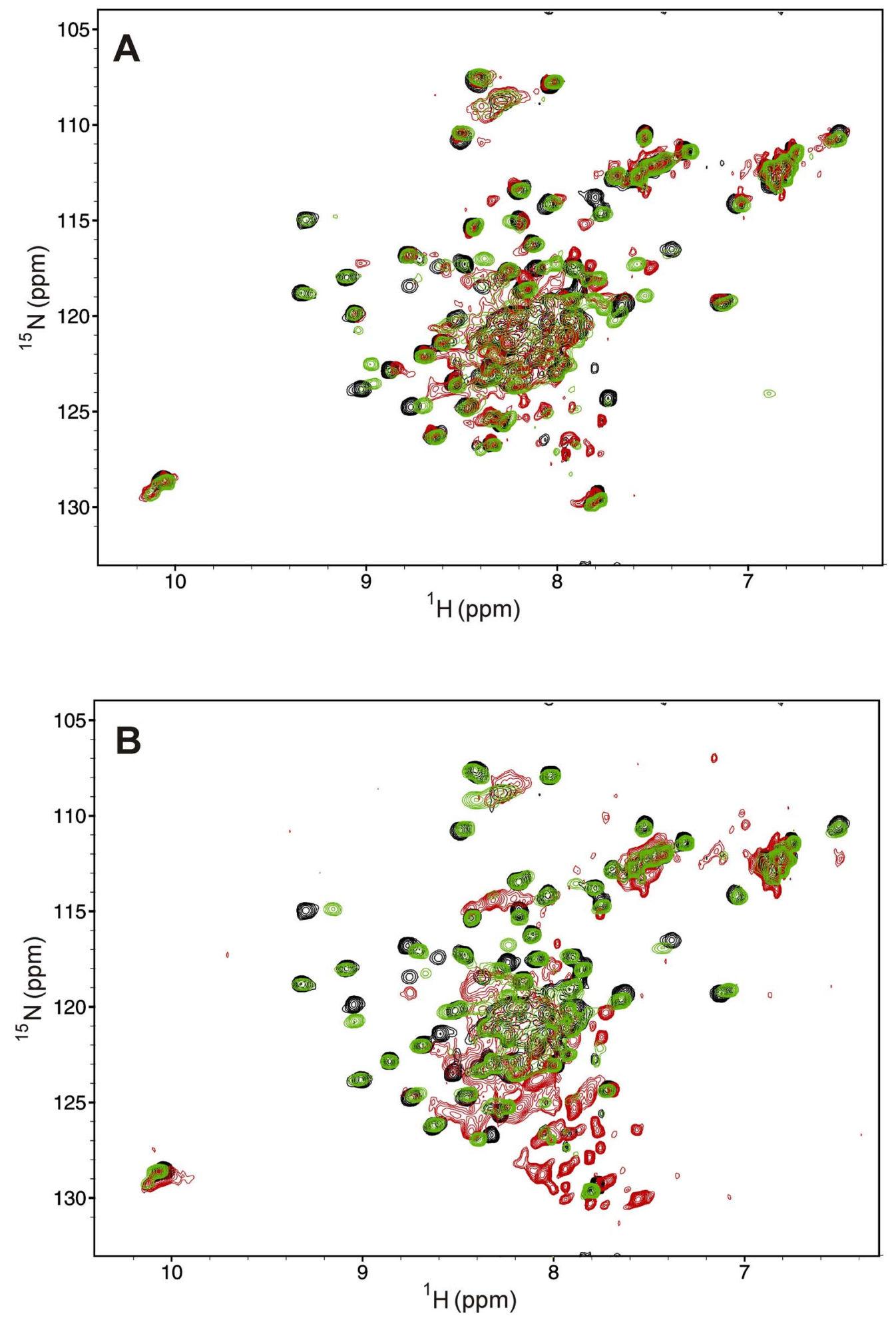

Figure 12. Evaluation of the deletional optimization of CBP-4 by $2 \mathrm{D}$ NMR. Superimposed ${ }^{15} \mathrm{~N}-$ HSQC spectra of the ${ }^{15} \mathrm{~N}$-uniformly labeled proteins; A: the wild type CBP-4 (black), CBP$4 \Delta(\mathrm{Gln} 168-A s p 174)$ (green) and CBP-4 $\Delta$ (Ser167-lle178) (red); B: the wild type CBP-4 (black), CBP-4 $\Delta$ (Leu216-Lys223) (green) and CBP-4 $\Delta$ (Gly213-Glu225) (red). 
localized either between Cys153 and Cys183: CBP-4 $\Delta(\mathrm{G} \ln 168-A s p 174)$ and CBP4 $\Delta$ (Ser167-Ile178), or Cys207 and Cys228: CBP-4L(Leu216-Lys223) and CBP$4 \Delta$ (Gly213-Glu225). The four mutants did no exhibit any improvement in the extent of folding over the wild type proteins, as determined by ${ }^{15} \mathrm{~N}-\mathrm{HSQC}$ spectra of the uniformly ${ }^{15} \mathrm{~N}$-labeled mutant proteins. On the contrary, determinants of unstructured regions were more pronounced, more so in the case of both longer deletion mutants (Figure 12). The inability of these constructs to form stable IGF-I/NBP-4/del-CBP-4 ternary complexes, as demonstrated by analytical gel filtration chromatography of the protein mixture indicated that either the global fold was disrupted by these deletions or/and residues important for IGF-I-binding were removed. The former is more likely as the probability of misfolded disulfide pairing in the $E$. coli recombinant proteins with abundant disulfide bridges is high (Rozhkova et al., 2004).

\subsection{Structure of IGFBP-4}

\subsubsection{Crystallization of the binary and ternary complexes}

Purity, homogeneity and folding of the proteins to be used in crystallization trials were evaluated using mass spectroscopy, amino-terminal sequencing and NMR. The three protein complexes, NBP-4(3-82)/IGF-I, NBP-4(1-92)/IGF-I and NBP-4(3-82)/CBP4/IGF-I, were prepared by mixing of approximately equimolar amounts of respective components. The complexes were separated from any excess of either protein by gel filtration on the Superdex 75 prep grade 26/60 column (Figure 13). A precise estimation of the protein ratio in the mixture was more important for the binary than the ternary complexes preparation. In the former case, excess of either NBP-4 or IGF-I in the sample would not be entirely separated from the binary complex using gel filtration (the difference of masses of binary complex and each of its components being not sufficient). The presence of uncomplexed proteins might negatively affect crystallization. For the ternary complex, addition of a slight molar excess of the Cdomain removed any remaining binary complex species in the mixture. Any surplus proteins were thus easily separated from the ternary complex (Figure 13, right panel).

Gel filtration chromatography enabled also thorough buffer exchange. The solvent used contained low salt $(50 \mathrm{mM} \mathrm{NaCl})$ and buffering substance ( $5 \mathrm{mM}$ Tris, $\mathrm{pH} 8.0)$ to 


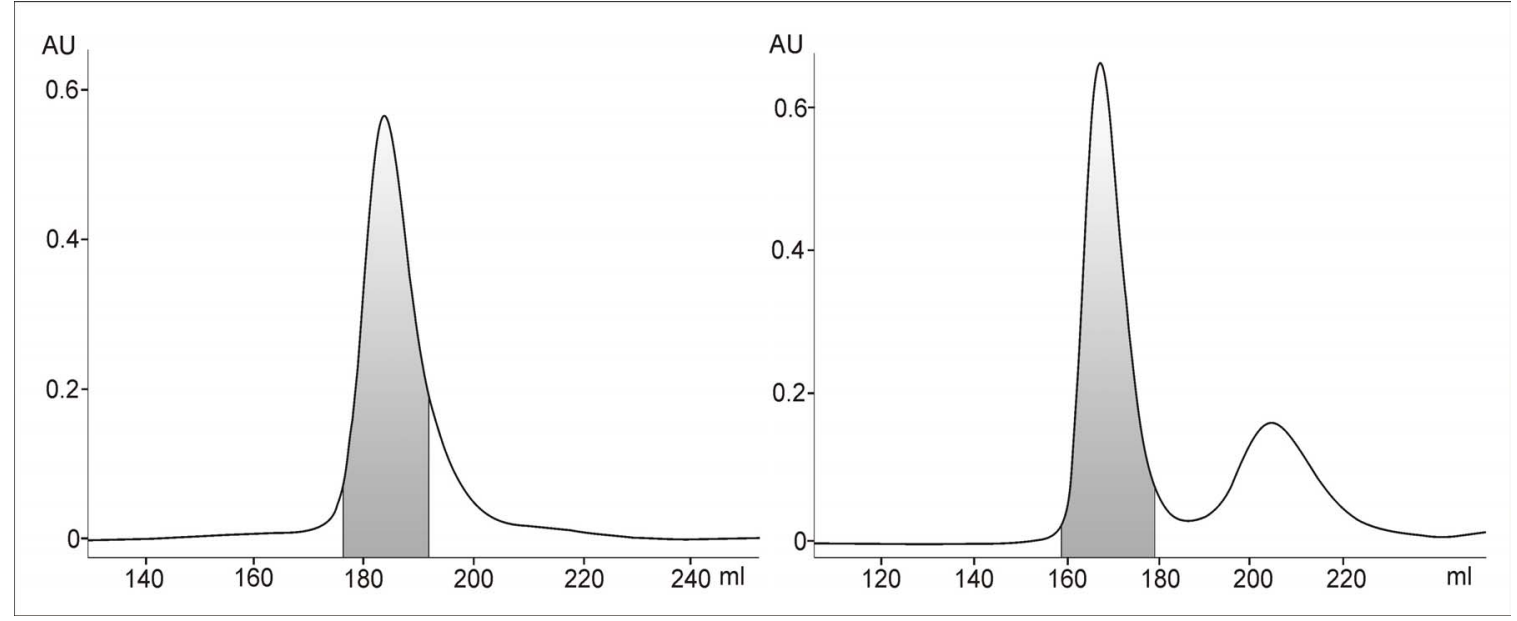

Figure 13. Purification of the complexed proteins by gel filtration on the Superdex 75 column. Right panel: NBP-4(3-82)/IGF-I binary complex purification; left panel: CBP-4/ NBP-4(382)/IGF-I ternary complex purification. Buffer used in both chromatographic runs: $50 \mathrm{mM} \mathrm{NaCl}$, $5 \mathrm{mM}$ Tris, $\mathrm{pH} 8.0,0.02 \% \mathrm{NaN}_{3}$; flow rate: $0.75 \mathrm{ml} / \mathrm{min}$; pooled fractions are indicated.

minimize any possible interference with crystallization reagents. Figure 14 shows photomicrographs of crystallization experiments described in detail in section 4.2.6.1, pp. 54-55.

\subsubsection{Overall structure of the NBP-4(3-82)/IGF-I binary complex}

Figure 15 shows the structure of the NBP-4 (3-82)/IGF-I complex. NBP-4 has an L-like shape and covers both the N- and C-terminal parts of IGF-I. Features of the NBP-4 structure itself will be discussed first; details of the IGF/BP-4 interactions are covered in a separate section below.

The global folds of NBP-4 (residues Ala39-Leu82) and miniNBP-5 (residues Ala40-Glu83) are almost identical in both complex structures, with main chain RMSD of $0.77 \AA$; the RMSD IGF/minNBP-5 vs. IGF/minBP-4 is $0.50 \AA$ (Figure $17 \mathrm{~A}$ ).

The core of the NBP-4(3-38) subdomain presents a novel fold stabilized by a short two-stranded $\beta$-sheet and 4 disulfide bridges forming a disulphide bond ladder-like structure (Figures 16 and $17 \mathrm{~B}$ ). This structure is connected to the miniNBP fragment only by a short stretch of amino acids, which include Ala39, Leu40 and possibly Gly41. The X-ray structure shows extensive interactions between NBP-4(3-38) and miniNBP-4 around this region. There are several hydrogen bonds between Arg50 and Ser53 of the NBP-4(3-38) subdomain and Glu23, Glu27, and Glu29 of the miniNBP-4 fragment. Additionally the linker residues make backbone hydrogen bonds to Cys77, Met42, 

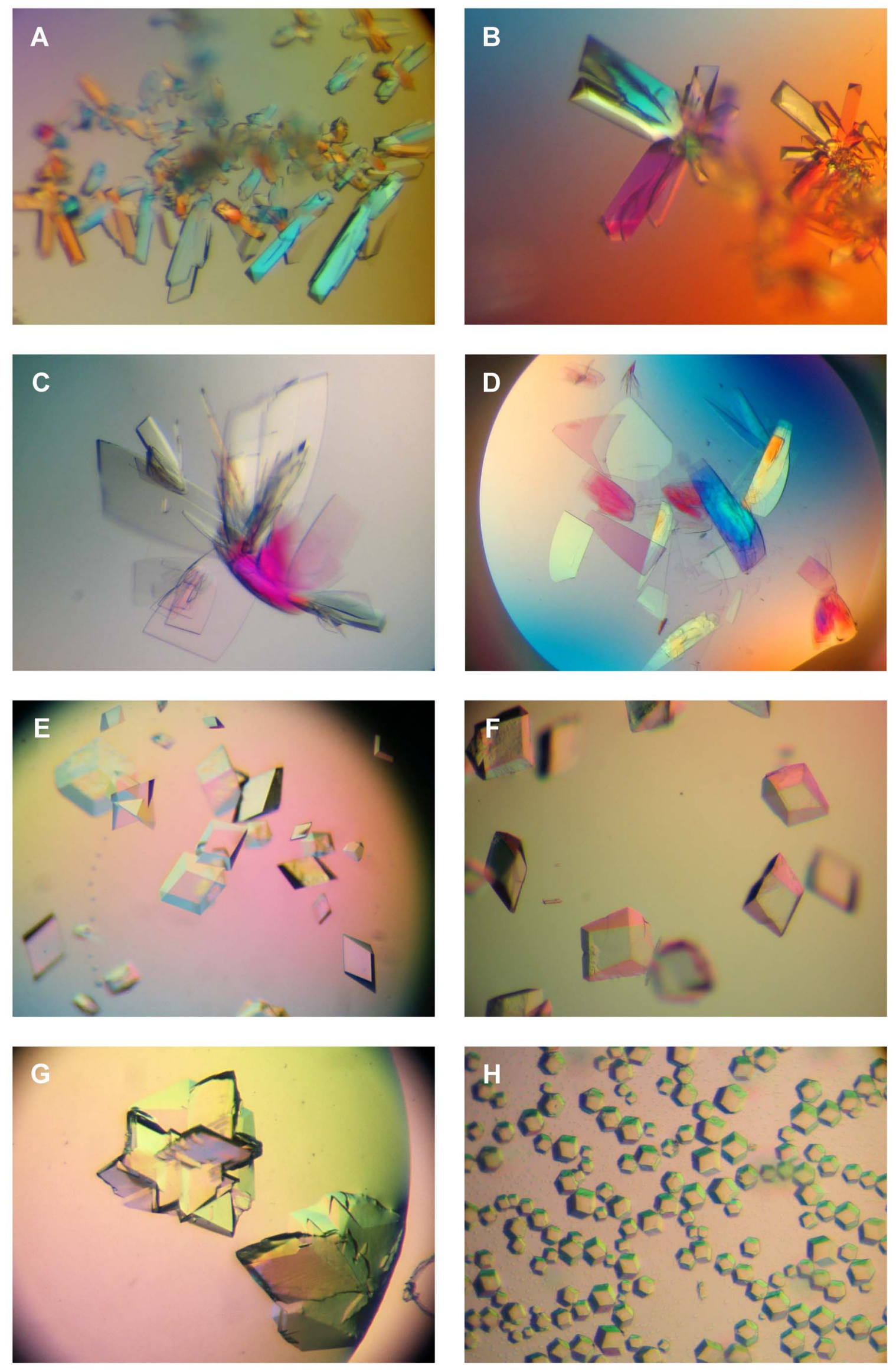
Figure 14. Examples of the crystallization experiments. A; B: X-ray quality orthorhombic crystals of the NBP-4(3-82)/IGF-I binary complex belonging to the P2 $2_{1} 2_{1}$ space group, grown from 23\% PEG 1500, $50 \mathrm{mM}$ Tris $\mathrm{pH}$ 8.2. The noticeable growth along a streak line arises from the micro-seeding technique used. C; D: the monoclinic plate crystals of the NBP-4(1-92)/IGF-I binary complex, obtained from $0.2 \mathrm{M}$ lithium sulfate, $0.1 \mathrm{M}$ Bis-Tris $\mathrm{pH} 5.5$ and $25 \% \mathrm{w} / \mathrm{v}$ PEG 3350 . The crystals belong to the $\mathrm{P} 2{ }_{1}$ space group. $\mathbf{E} ; \mathbf{F}$; $\mathbf{G}$ : rhombohedral crystals of the CBP4/ NBP-4(3-82)/IGF-I ternary complex that appeared in $1.0 \mathrm{M}$ lithium sulfate, $1 \% \mathrm{w} / \mathrm{v}$ PEG 8000. Space group: R32. H: the ternary complex crystals from $1.0 \mathrm{M}$ lithium sulfate, $15 \% \mathrm{w} / \mathrm{V}$ PEG 8000.

\section{A}
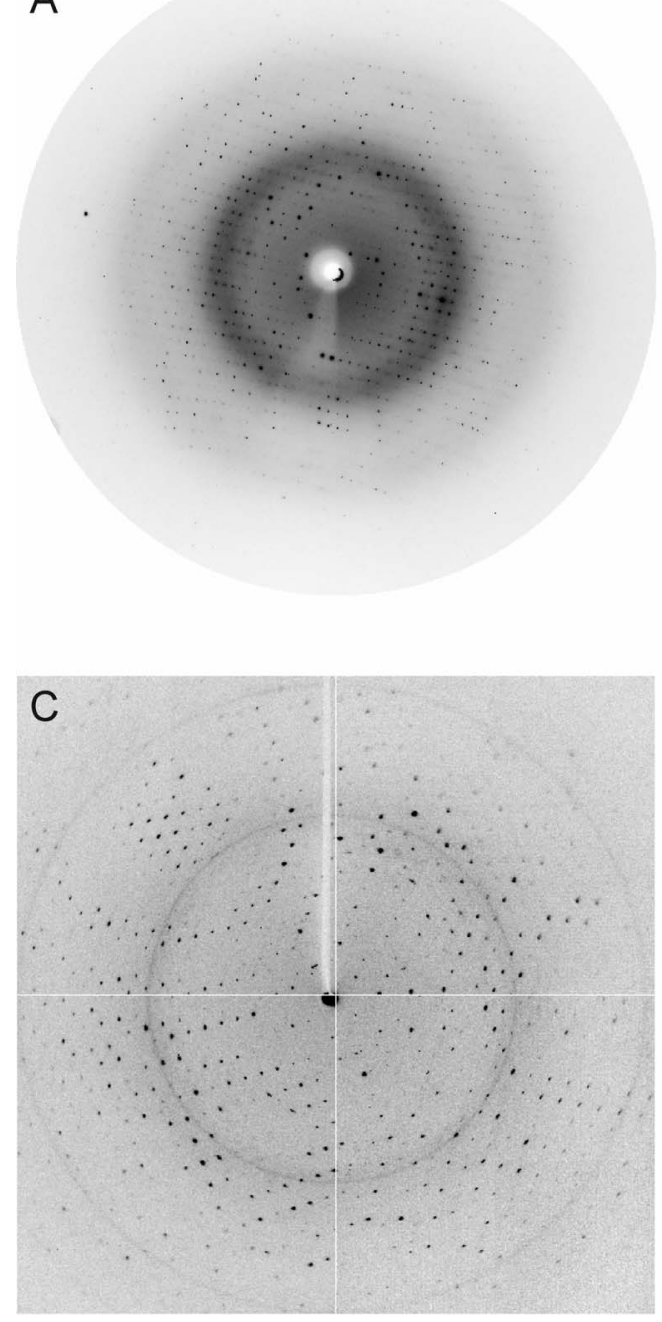
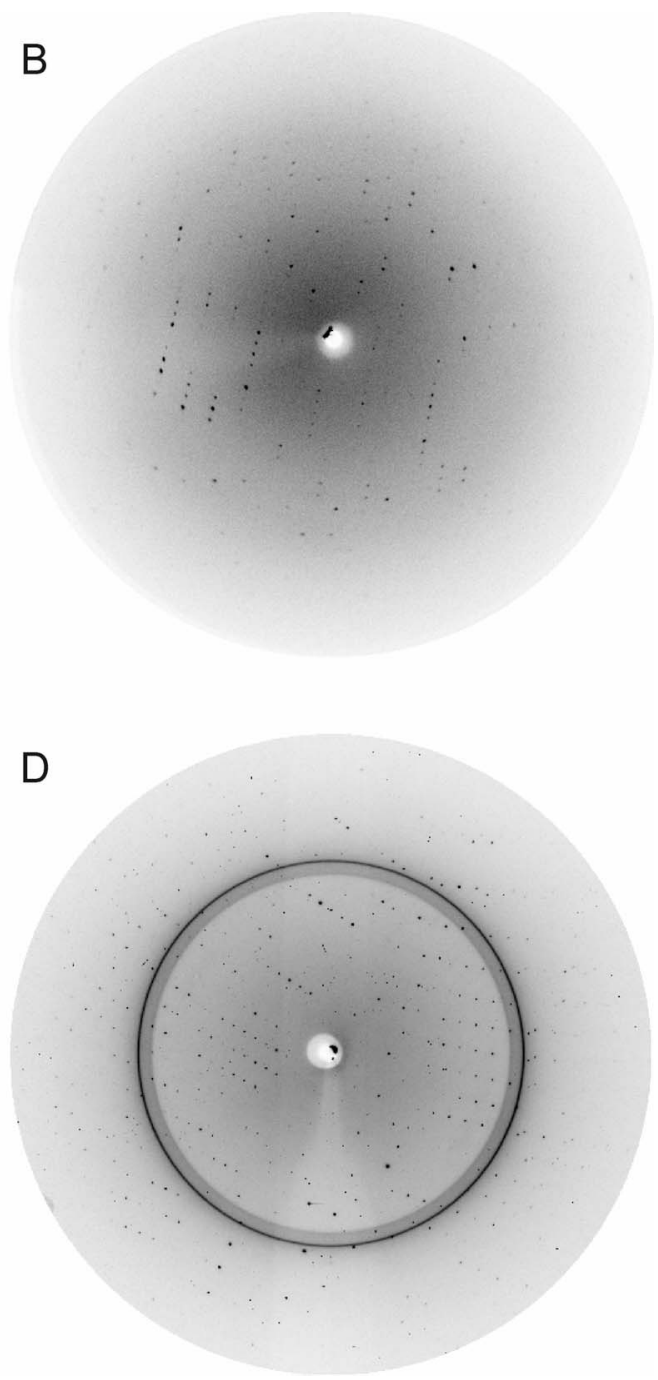

Figure 15. Diffraction patterns of crystals of the binary and ternary complexes. A: A frame from the MAR CCD165 (BW6, DESY, Hamburg). The crystal of NBP-4(3-82)/IGF-I (space group $\mathrm{P} 2{ }_{1} 2_{1} 2_{1}$ ) was rotated $2^{\circ}$. The edge of the image is about $1 \AA$. $\mathbf{B}$ : A frame from the MAR 345 image plate, in-house rotating copper anode $x$-ray source. The $P 2{ }_{1}$ crystal of NBP-4(1-92)/IGF was rotated $3^{\circ}$. Resolution at the edge of the detector is ca. $1.5 \AA$. C: A diffraction image from the ADSC Q210 detector (ID29 beamline, ESRF, Grenoble, France). The C2 crystal of the ternary NBP-4(3-82)/CBP-4/IGF-I complex was rotated $2^{\circ}$. D: The diffraction pattern of the R32 crystal of NBP-4(3-82)/CBP-4/IGF-I complex from the MAR CCD165 (BW6, DESY, Hamburg). Resolution at the edge of the detector is ca. $2.8 \AA$. 

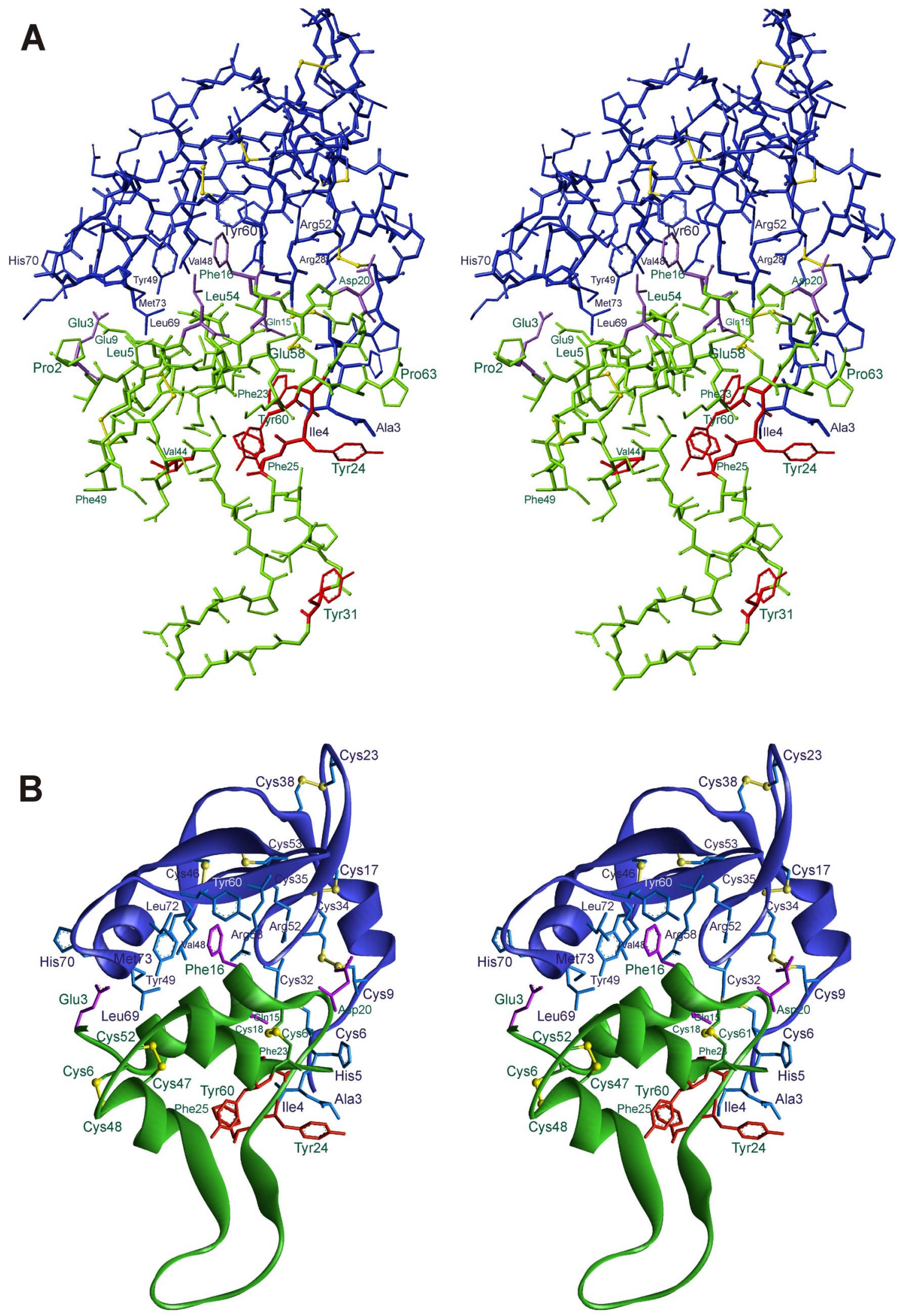
Figure 16. Structure of the NBP-4(3-82)/IGF-I complex. Heavy atom (A) and ribbon (B) plots of the binary complex. NBP-4 is shown in blue, IGF-I in green. Residues shown in violet constitute the binding site for interaction with NBP-4. Residues marked in red are determinants for binding to IGF-IR.

Gly52 and Arg50 of NBP-4(3-38). This network of H-bonding assures high degree of rigidity. The two subdomains are perpendicular to each other, creating the " $\mathrm{L}$ " shape for the whole N-domain. The miniNBP is globular, whereas in NBP-4(3-38) the $\beta$-sheet and disulfide bridges are all in one plane making the structure appear flat from one side. The core of the NBP-4(3-38) subdomain can be therefore visualized as a "palm" of a hand. The palm is extended with a "thumb" segment of 4-12 residues in various IGFBPs. The thumb segment consists of the very $\mathrm{N}$-terminal residues up to the equivalent of Cys6 in IGFBP-4 and contains a consensus XhhyC motif, where $h$ is a hydrophobic amino acid and y is positively charged.

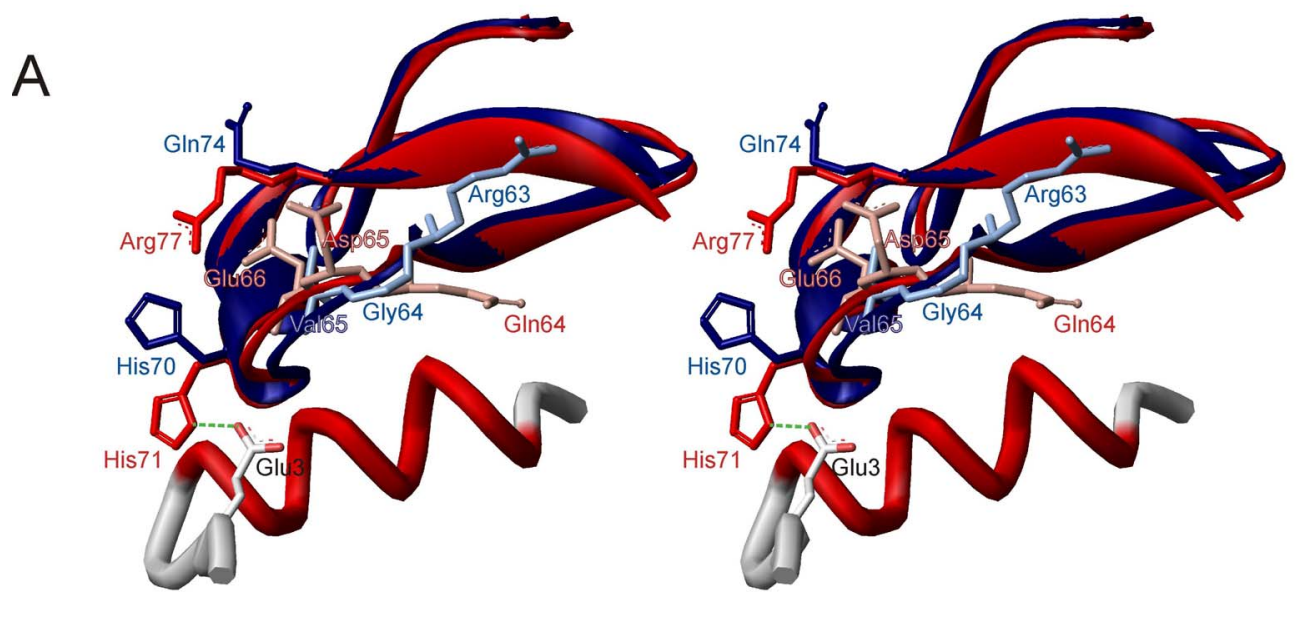

B
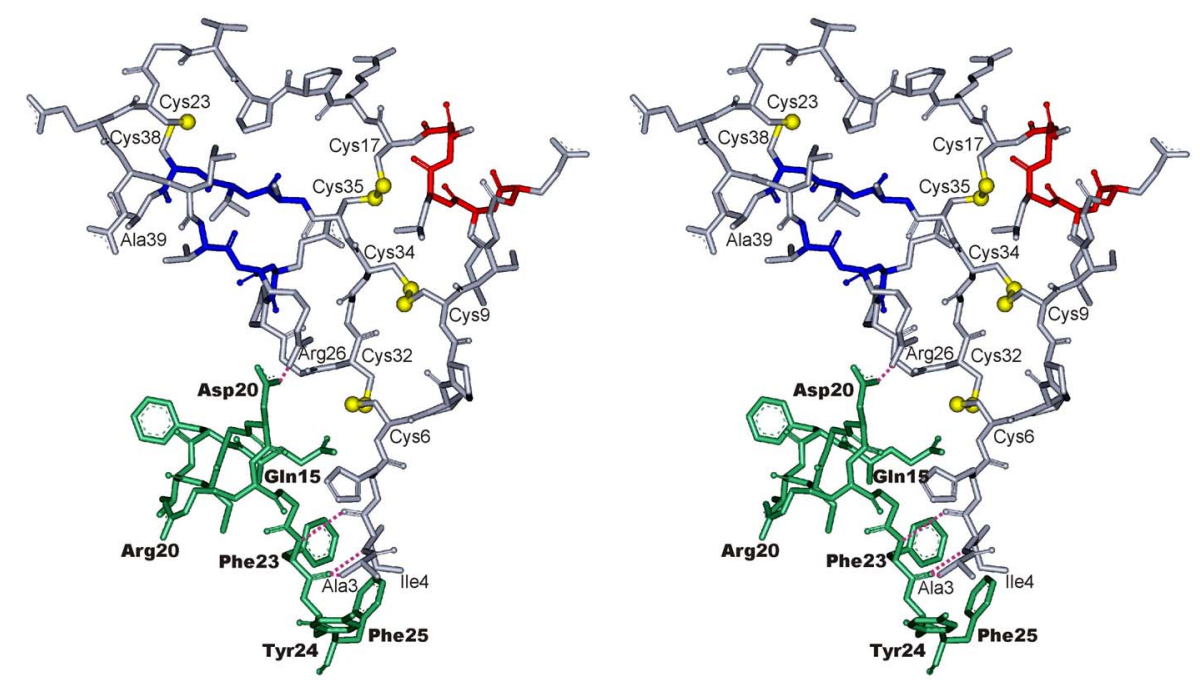
Figure 17. Structures of the NBP-4(3-82)/IGF-I complex. A: Comparison of miniNBP-5 and miniNBP-4 interactions with IGF-I. MiniNBP-5 (red) and miniNBP-4 (blue) were fitted with Swiss-PdbViewer 3.7. B: The structure of the N-terminal subdomain of NBP-4 and its interaction with IGF-I. Residues Ala3-Ala39 of IGFBP-4 are colored in gray, with secondary structure elements in blue (for $\beta$-sheet) and red (for $\alpha$-helix). The IGF-I fragment (GIn15Phe25) is in green. The planar arrangement of disulphide bridges (yellow) is clearly seen.

The structure of IGF-I shown here is of the highest resolution to date (1.6 $\AA$ ). Only very recently have X-ray structures of IGFs been published, with resolutions ranging from 1.8-2.5 $\AA$ (Vajdos et al., 2001; Brzozowski et al., 2002). In our structure a fragment of IGF-I consisting of amino acids Gly32-Gly42 is clearly seen in the electron density map. This segment was not previously defined but had apparently retained a natural flexibility in the crystal form in agreement with NMR data for free IGF-II (Torres et al., 1995). The fragment assumes a $\beta$-hairpin-like structure and appears to be stabilized by crystal packing, as several hydrogen bonds are present with neighboring NBP-4 molecules in the unit cell.

\subsubsection{NBP4(3-82)/IGF-I vs. NBP4(1-92)/IGF-I}

The first 92 residues of IGFBP-4 are $59 \%$ identical to the corresponding N-terminal residues of IGFBP-5, and the remaining residues are mostly functionally conserved. For miniNBP-5 (residues 40-92), the last 9 amino acids showed no electron density in its IGF complex structure (Zeslawski et al., 2001) and were unstructured as determined by NMR (Kalus et al., 1998). Equivalent residues therefore were not expressed in the construct NBP-4(3-82) to aid crystallization of the complex. However, residues Glu90 and Ser91 of IGFBP-4 were reported to be significant for high affinity binding with IGFs (Qin et al, 1998), and therefore we decided to include these residues in our extended $\mathrm{N}$-terminal construct. The two first $\mathrm{N}$-terminal residues were also added because the IGFBP-4(3-82)/IGF-I structure revealed the importance of the two N-terminal hydrophobic residues conserved among IGFBPs. Possibility also existed that eliminating the two first negatively charged residues, Asp1 and Glu2, at the N-terminus in the IGFBP-4 could have changed the properties of this amino terminal part. These residues were therefore added to the refined N-terminal construct, generating NBP4(1-92).

Examination of the structures of the two complexes reveals virtually no changes in positions of the backbone and most of the side chain atoms of both NBP-4 and IGF heavy atoms RMSD of $0.75 \AA$. It can therefore be concluded that the presence of 

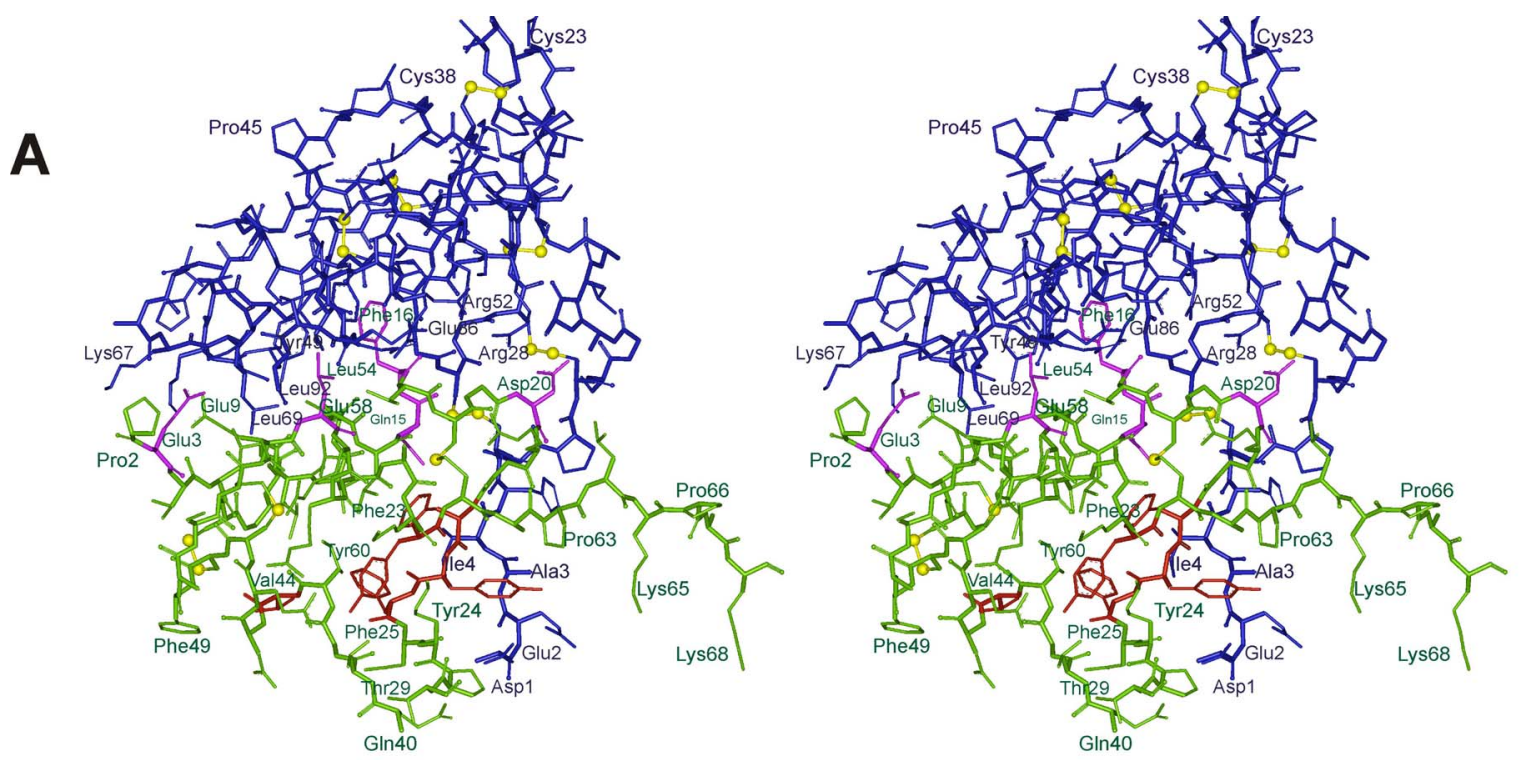

B
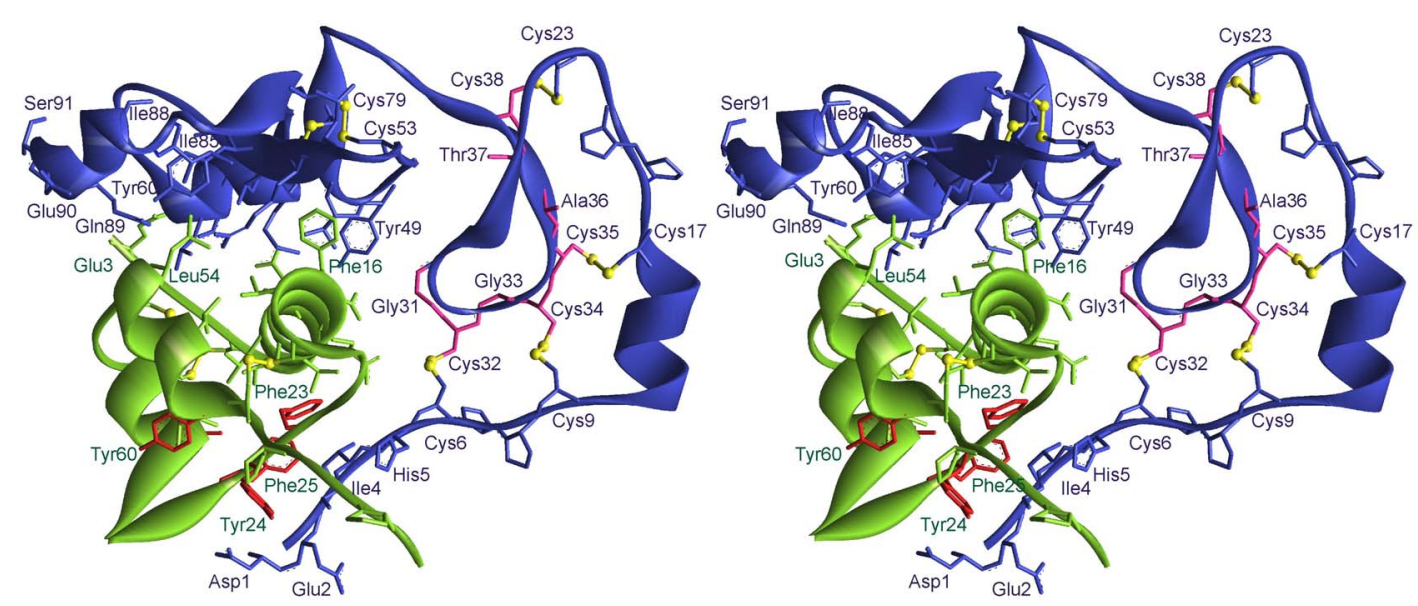

C
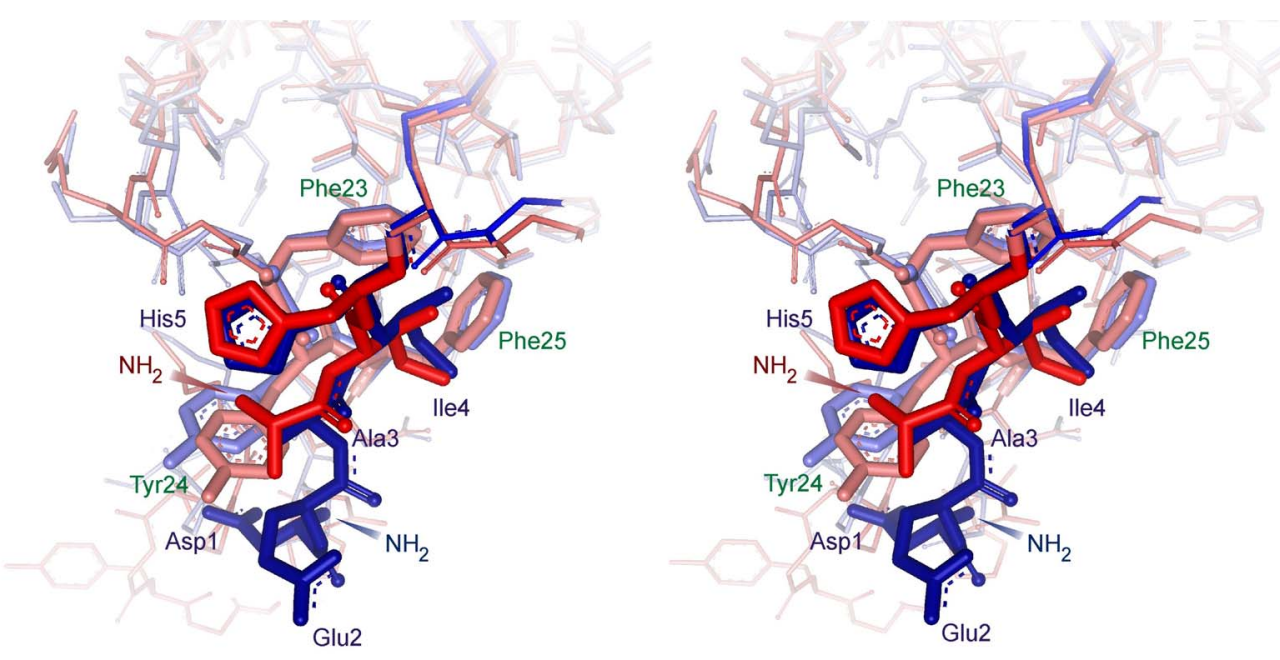
Figure 18. Structure of the NBP-4(1-92)/IGF-I complex. Heavy atom (A) and ribbon (B) plots of the complex. NBP-4 is shown in blue, IGF-I in green. Residues shown in violet constitute the binding site for interaction with NBP-4. Residues marked in red are determinants for binding to IGF-IR. The GCGCCXXC consensus motif is shown in magenta (B). Comparison of the IGF-I residues interacting with the "thumb" region in complex with NBP-4(3-82) (red) and NBP-4(192) (blue) (C).

residues 1, 2 and 83-92 does not influence the fold of NBP-4 or IGF-I (Figure $18 \mathrm{C}$ ). Complexes of a longer and shorter NBP-4 with IGF-I crystallized in two different space groups; identity of the two structures therefore proves that crystal packing has no influence on their folds. Crystal packing, however, had influence on the loose parts of the IGF-I molecule: the flexible loop spanning residues $30-40$, clearly defined in the NBP-4(3-82)/IGF-I, showed no electron density in NBP-4(1-92)/IGF-I.

The sequence Ala83-Leu92, of which the fragment Glu84-Glu90 forms a short helix, does not contact IGF directly. In the study of Qin et al. (1998), deletion of Glu90 and Ser91 led to the reduced IGF-I and -II binding activity, suggesting functional significance of these residues. Our crystallographic structure, however, shows no contribution of these two residues in formation of the IGF binding site. The presence of the 10-amino acid-long fragment may have, however, an indirect influence on IGF binding: side chains of Ile85, Ile88, and GIn89 shield Tyr60 side chain from the solvent and constrain its conformation of Tyr60 that otherwise would point away from the IGF surface, as can be seen in the NBP-4(3-82)/IGF-I complex structure. Tyr60 along with Pro61 form small hydrophobic cleft, in which Leu54 of IGF-I is inserted, thus extending the hydrophobic contact area of the two proteins.

The position of the His70 side chain in NBP-4(3-82) was rotated ca. $180^{\circ}$ relative to the corresponding His71 of miniBP-5. In the structure of NBP-4(1-92), the imidazole ring of the histidine is however flipped back to the configuration observed in the miniNBP-5/IGF-I complex and forms a network of hydrogen bonds with side chains of Glu3 and Glu9 of IGF-I. Similarly, in the NBP-4(3-82)/IGF-I complex structure, IGFs' Phe16 was found rotated ca. $20^{\circ}$ clockwise about the $\alpha$-helix axis, when compared to its position in the miniBP-5/IGF-I complex. In the structure of IGF-I bound to NBP-4(192), Phe16 returns to the position found in the miniNBP-5/IGF-I.

\subsubsection{The subdomain organization of NBP-4}

The N-terminal domain, NBP-4, can be divided into two subdomains: the fragment from residues Asp1-Cys39 and the segment Met44-Glu90. The latter can exist 
autonomously and corresponds approximately to the mini-IGFBP-5 described by Kalus et al. (1998). The two subdomains are connected by a short stretch rich in Gly and Leu (i.e. Ala39, Leu40, Gly41, Leu42, and Gly43). In order to assess the flexibility of this short stretch, a selectively labelled ${ }^{15} \mathrm{~N}$-Gly and ${ }^{15} \mathrm{~N}$-Leu sample of NBP-4 has been prepared and then a ${ }^{1} \mathrm{H}_{-}{ }^{15} \mathrm{~N}$ het-NOE experiment was performed. The NOE values obtained for these residues are shown in the Figure 19. It is seen from the figure that only one peak is below the threshold level of the NOE intensity 0.5 , indicating that the linker is rigid. This one peak can be assigned with high probability to Gly56 present in the loop known to be flexible from mini-NBP-5 NMR studies.

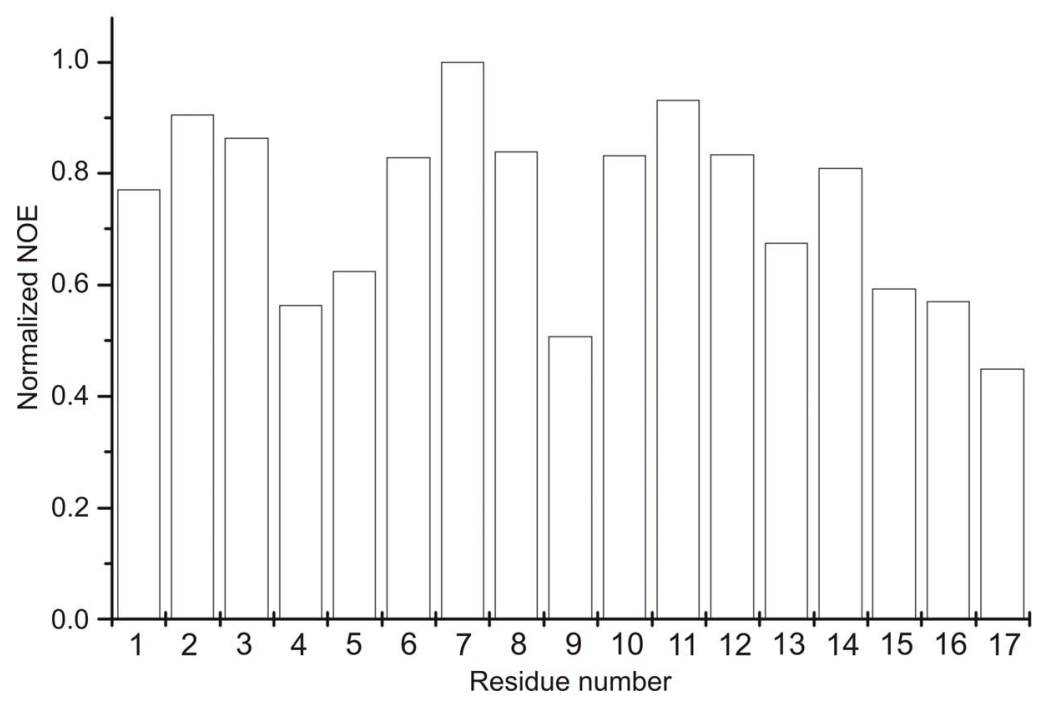

Figure 19. NOE intensity values for all glycine and leucine residues of NBP-4(3-82). The numbers have been assigned randomly.

\subsubsection{Ternary complex NBP-4(3-82)/IGF-I/CBP-4(151-232)}

We have also obtained crystals of a ternary complex of NBP-4(3-82) and the Cterminal domain, CBP-4(151-232), with IGF-I (Table 4). The structure of the binary segment NBP-4(3-82)/IGF-I in this complex could be readily solved by molecular replacement. Unfortunately, the C-domain produced electron densities in disconnected patches that could not be interpreted in detail. Only fragments of the domain in contact with or in proximity to NBP-4/IGF-I showed electron density sufficiently defined to allow incorporation of fragments of a polyalanine model. Identities of individual residues could not however be reliably established. The data allows identification of the Cdomain interface surface of IGF-I and NBP-4 (Figures 20, 21 and 30). CBP-4 appears 
to exhibit a flat structure that has far-reaching interactions with the $\mathrm{N}$ terminal part of NBP-4 from Ala3 up to Tyr48 and also extensive contacts with IGF-I.
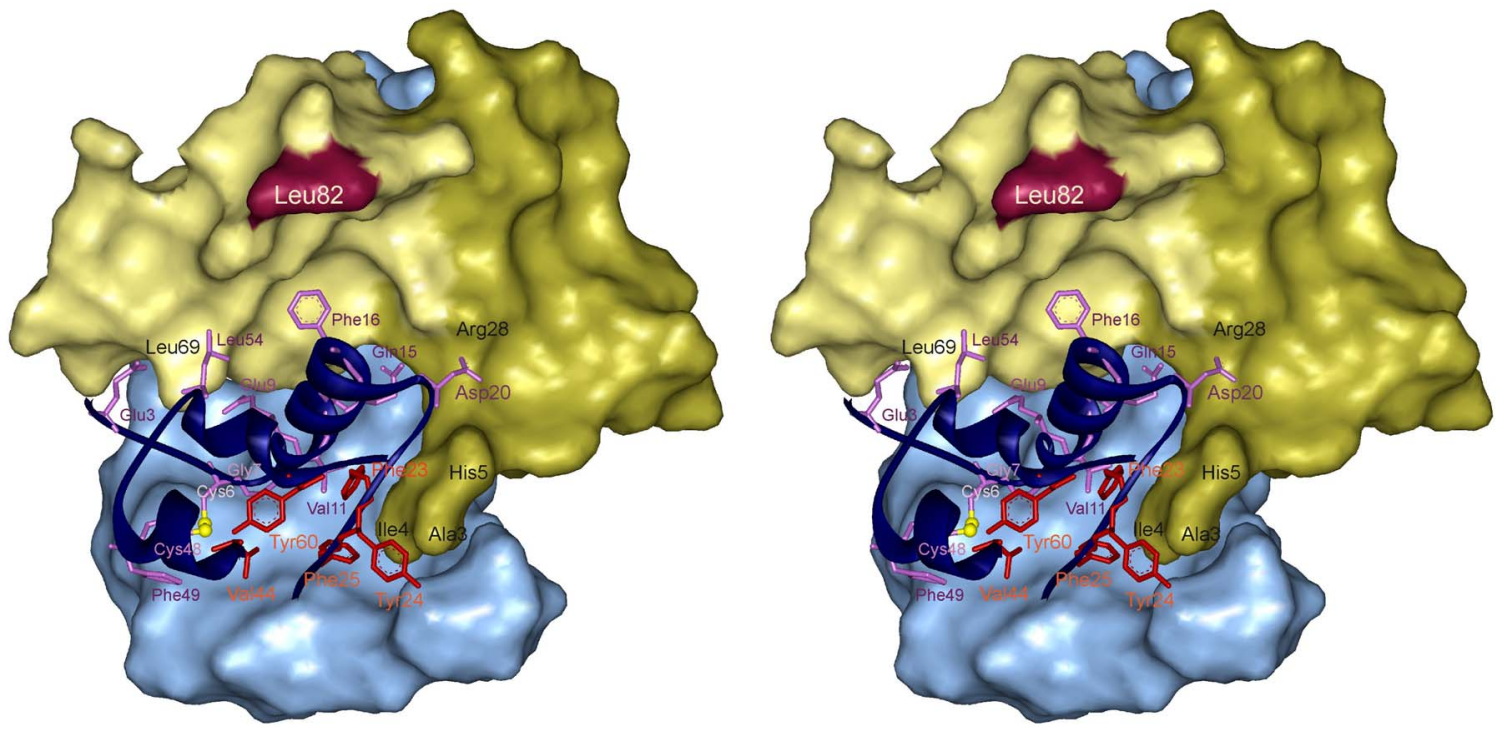

$\mathbb{B}^{90^{\circ}}$
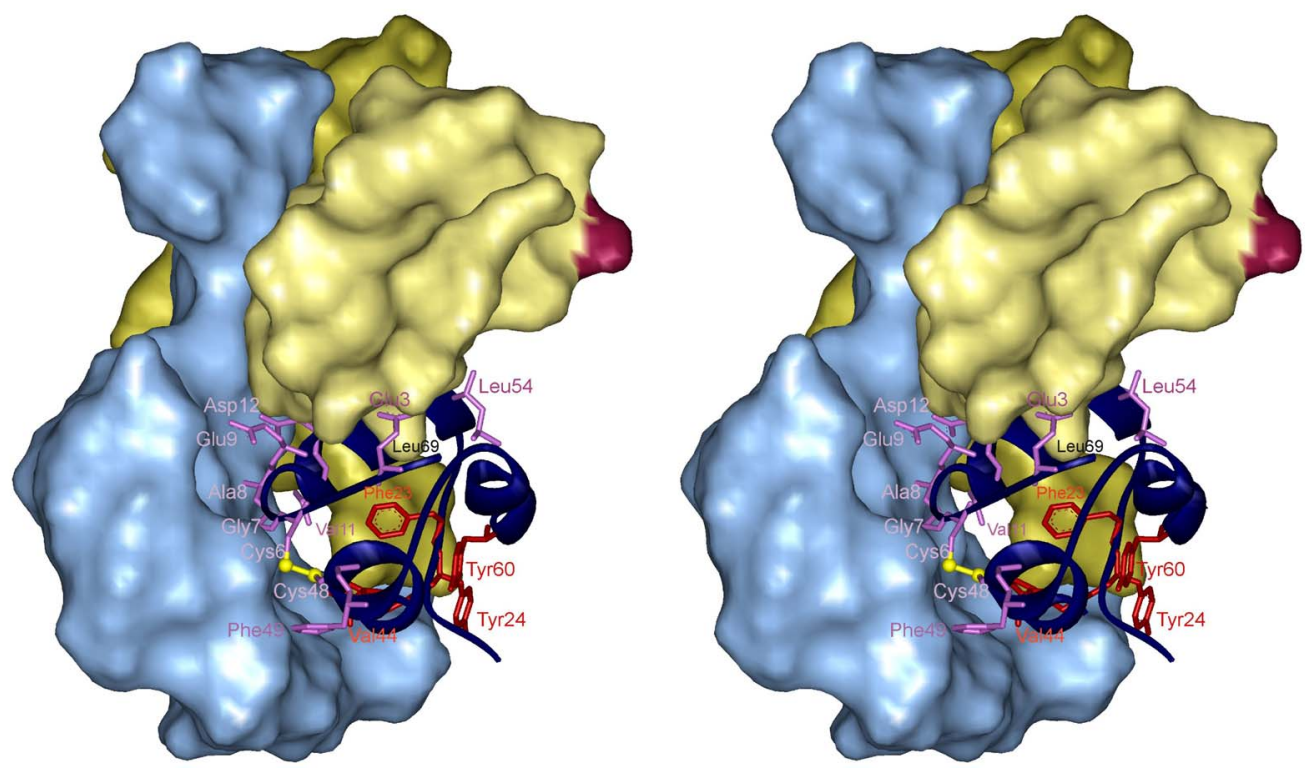

Figure 20. Surface plot of the structure of the IGF-I/CBP-4/NBP-4 ternary complex. Sidechains of IGF residues important for IGF-IR binding are shown in red. Primary determinants of the IGF/IGFBP-4 interaction are shown in violet. The "base" region of NBP-4 is colored in lighter, "palm"-"thumb" in darker shades of yellow, IGF-I (dark blue ribbon); key IGF residues are numbered, IGF residues responsible for IGF-IR binding are shown in red; CBP-4 is presented as a light blue surface. 

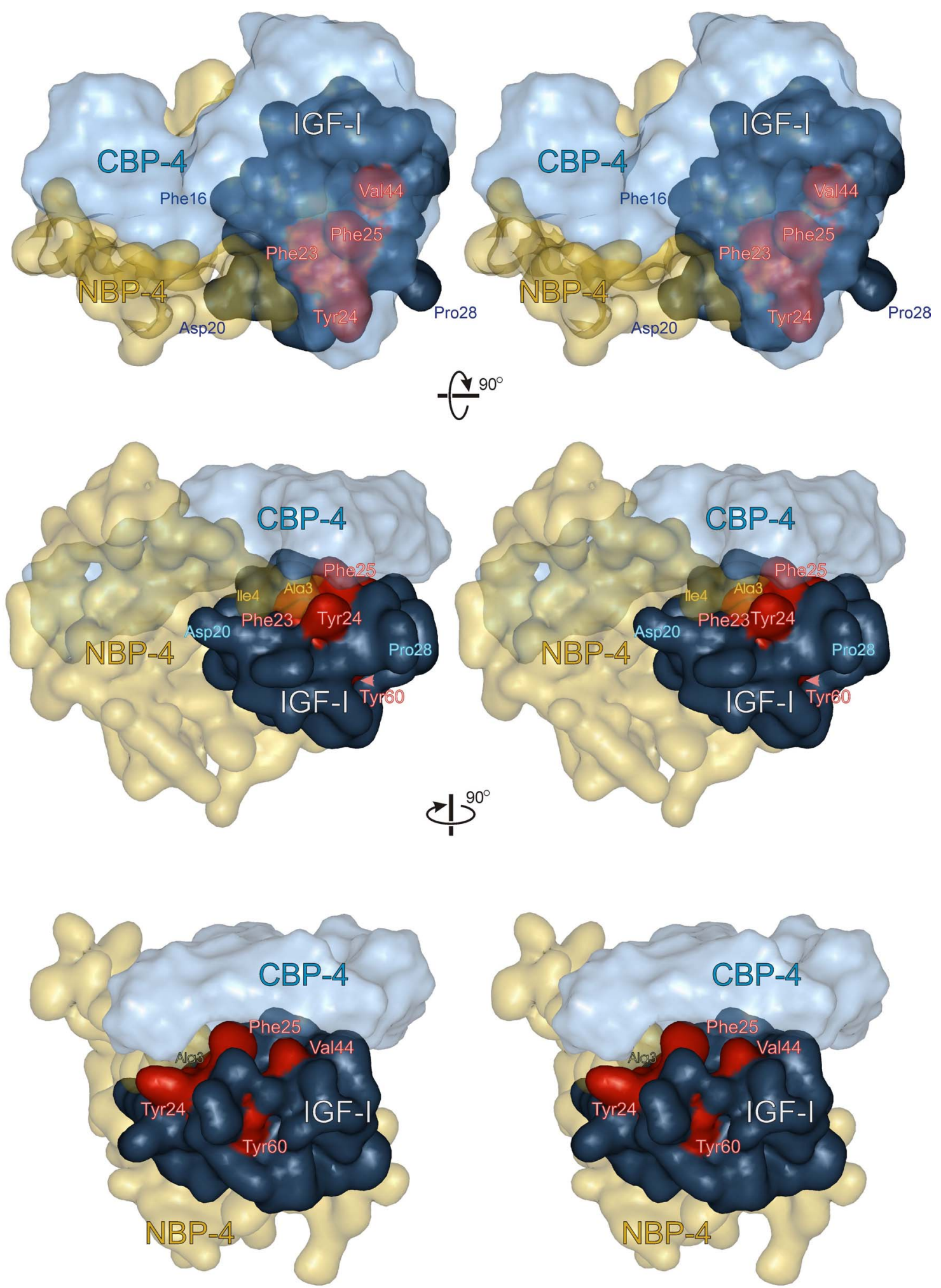

Figure 21. A surface plot of the structure of the IGF-I/CBP-4/NBP-4 ternary complex. IGF-I (solid dark blue); key IGF residues are numbered, IGF residues responsible for IGF-IR binding are shown in red; the NBP-4's transparent surface is shown in yellow; CBP-4 is presented as a transparent light blue surface. 


\subsubsection{The IGF-I/NBP-4 interaction - implications for IGF-I binding to its receptor}

The principal interface between IGF-I and NBP-4 is located in the miniNBP subdomain and comprises a hydrophobic sandwich that consists of interlaced protruding side chains of IGF-I and solvent-exposed hydrophobic side chains of the NBP-4. The side chains of IGF-I Phe16 and also Leu54 are inserted deep into a cleft present in NBP-4. This cleft is formed by side chains of Arg52 and Arg58 on one side of the molecule and by Val48, Leu69 and Leu72 on the opposite inner side, with a base formed by residues Gly47, Cys59 and Tyr60. Phe16 of IGF makes direct contacts with the backbone and side chain of Val48, and with Cys59 of NBP-4. The IGF Phe16 NBP-4 interaction is closed on the solvent side by side chains of Glu3 and Glu9 of IGF-I, and by Tyr49, Thr50 and Pro30 of NBP-4.

The N-terminal palm subdomain (between residues 6-38) and the thumb segment (residues 3-5) make several additional contacts to IGF-I. The "thumb" Ala3 and lle4 of NBP-4 make major interactions by filling the hydrophobic patch created by IGF Phe23, Tyr24 and Phe25. The interaction is further strengthened by hydrogen bonding (lle4 $\mathrm{C}=\mathrm{O}$ to Phe23 $\mathrm{NH}$, lle4 $\mathrm{NH}$ to Phe23 $\mathrm{C}=\mathrm{O}$ ). The palm contacts IGF only from one edge mostly through BP-4 Arg28 (Arg28 NH1 to the IGF Asp 20 OD1).

The C-domain appears to make extensive contacts to IGF. Contact residues on IGF are Cys6-Ala8, the sidechain of Glu9, Val11 and Asp12, Tyr24, Phe25, Asn26, Val44, Cys48 and Phe49 (Figures 19, 20 and 30).

\subsection{Functional studies of IGFBP-4 - IGF interaction}

\subsubsection{Binding among domains of IGFBP-4 and IGF as studied with NMR and ITC measurements}

ITC calorimetry indicates that NBP-4(3-82) and NBP-4(1-92) bind IGF-I with $\mathrm{K}_{\mathrm{D}} \mathrm{S}$ of ca. $0.8 \mu \mathrm{M}$ and $0.3 \mu \mathrm{M}$, respectively (Table 7; the ITC graphs can be found in the Appendix 2). The presence of the C-terminal domain (CBP-4, residues 151-232) increases affinities of both NBP-4s to IGF-I, resulting in a 2-6 times tighter binding. IGF-I-binding activities of both NBP-4(3-82) and NBP-4(1-92) in the presence of CBP-4 are comparable and close to the miniNBP-5 affinity alone (without CBP-4). CBP-4 does not influence IGF-I binding activity of miniNBP-5 (see below). No direct binding between CBP-4 and NBP-4, and CBP-4 and IGF-I could be detected. 
Table 7. ITC Data

\begin{tabular}{lll}
\hline Reservoir & Titrant & $\mathrm{K}_{\mathrm{D}} \pm \mathrm{SD}[\mathrm{nM}]$ \\
\hline & \multicolumn{2}{c}{ IGFPB-4 } \\
\hline NBP-4 & IGF-I & $865 \pm 78$ \\
IGF-I & NBP-4 & $653 \pm 64$ \\
miniNBP-4 & IGF-I & $3020 \pm 410$ \\
IGF-I + CBP-4 & NBP-4 & $266 \pm 28$ \\
CBP-4 + NBP-4 & IGF-I & $147 \pm 27$ \\
NBP-4 + IGF-I & CBP-4 & $\mathrm{K}_{1}=450 \pm 100, \mathrm{~K}_{2}=20000 \pm 5000$ \\
CBP-4 & NBP-4 + IGF-I & $\mathrm{K}_{1}=178 \pm 75, \mathrm{~K}_{2}=1050 \pm 200$ \\
NBP-4 (1-92) & IGF-I & $311 \pm 17$ \\
NBP-4(1-92) + CBP-4 & IGF-I & $140 \pm 23$ \\
& & \\
\hline & & $153 \pm 31$ \\
NBP-5(1-94) & IGF-I & $209 \pm 34$ \\
miniNBP-5 & IGF-I & $125 \pm 61$ \\
IGF-I & miniNBP-5 & $113 \pm 12$ \\
IGF-I + CBP-4 & miniNBP-5 & $136 \pm 29$ \\
CBP-4 + miniNBP-5 & IGF-I & $3440 \pm 600$ \\
miniNBP-5 + IGF-I & CBP-4 & $2040 \pm 370$ \\
CBP-4 & miniNBP-5 + IGF-I & $443 \pm 58$ \\
IGF-I & miniNBP-5-L74M & $145 \pm 33$ \\
IGF-I & miniNBP-5-L61Y & \\
\hline & & \\
\hline
\end{tabular}

In order to exclude any interaction that could be weak, these interactions has been tested by NMR using ${ }^{15} \mathrm{~N}$ labeled CBP-4 or NBP-4. Addition of CBP-4 did not cause any changes in the ${ }^{15} \mathrm{~N}-\mathrm{HSQC}$ spectrum of ${ }^{15} \mathrm{~N}$ labeled NBP-4, proving no interaction. In a similar manner, addition of IGF-I to ${ }^{15} \mathrm{~N}-\mathrm{CBP}-4$ had no effect on the ${ }^{15} \mathrm{~N}$ HSQC. In a positive control we were able to see clearly the interaction of NBP-4/IGF-I with ${ }^{15} \mathrm{~N}$ CBP-4 (Figure 22). Ligand binding-induced folding of partially unstructured proteins and peptides is an often-observed phenomenon (Weber et al., 2000). The experiments described above did not reveal such a behavior for CBP-4: no cross peaks emerged from the ${ }^{1} \mathrm{H}$ 8.3-ppm spectral region after addition of NBP-4/IGF-I (Figure 22). Similarly, 1-D spectra of the full-length IGFBP-4/IGF-I complex do not show any improvement in terms of folding over free IGFBP-4 (Figure 23). These experiments 
show that neither carboxyl-terminal unstructured regions nor the linker domain are influenced by complex formation, and the overall folding pattern of IGFBP-4 is unaffected by IGF-I binding.
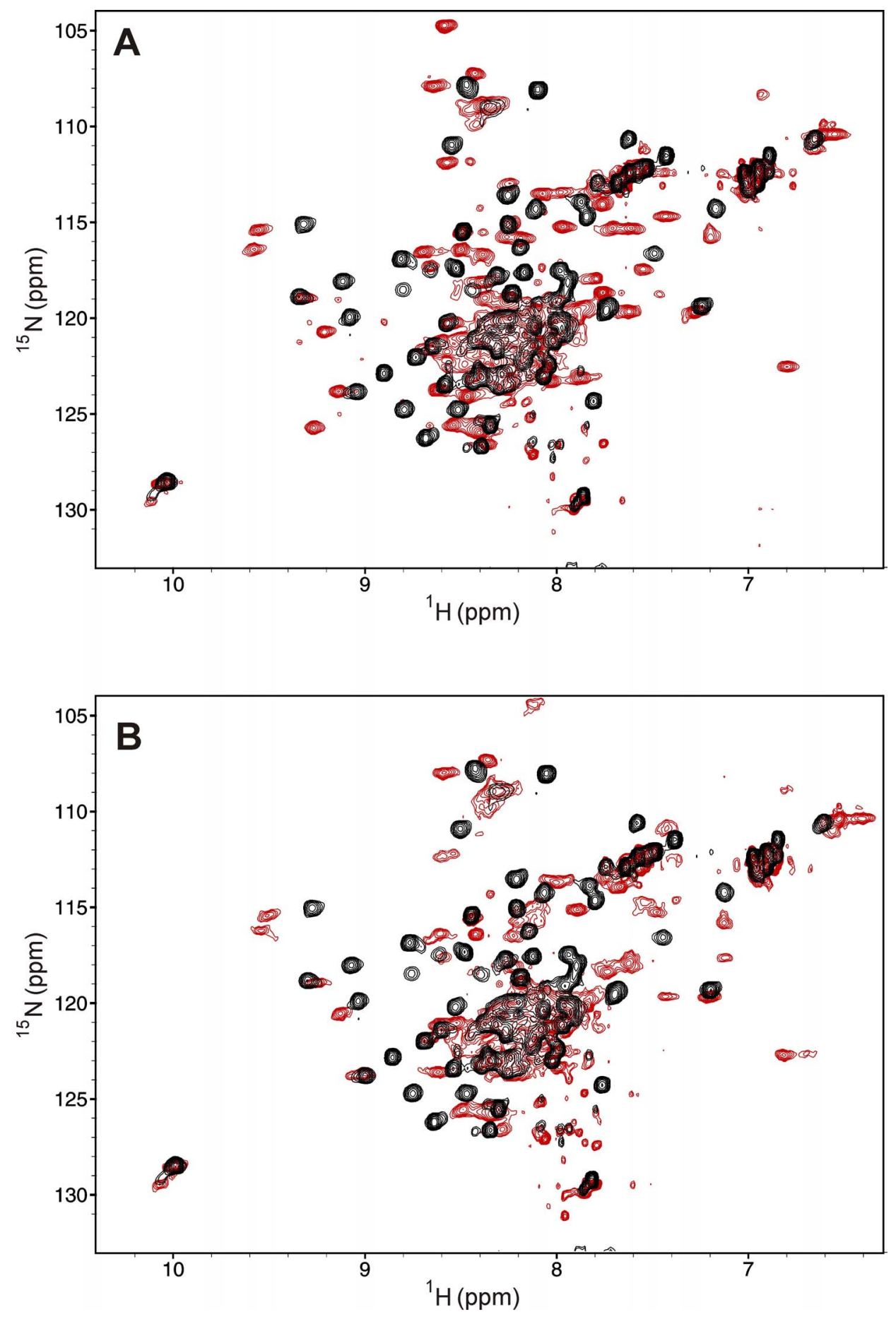

Figure 22. Effect of the ternary complex formation on CBP-4 folding. Superimposed 2DHSQC spectra of the ${ }^{15} \mathrm{~N}$-labeled CBP-4 in a free form (black) and bound to (A) the NBP-4(382)/IGF-I binary complex (red), and (B) the miniNBP-5/IGF-I binary complex. 
Binding of CBP-4 to a preformed binary complex of NBP-4/IGF-I shows two site kinetics, with initial titration of the stronger, higher affinity site and subsequent binding to the lower affinity site (Table 7). Titration of CBP-4 into NBP-4/IGF-I produced low reaction heat values and consequently a large $\chi^{2}$ value, hence the deduced dissociation constants have to be regarded as approximate. CBP-4 does not influence the parameters of miniNBP-5 binding to IGF-I. It seems that binding of CBP-4 to the preformed binary complex of NBP-4/IGF-I follows a different mechanism than binding to the miniNBP-5/IGF-I binary complex. The kinetics of the latter reaction fit the one site model, while two binding site kinetics are required to describe the former. MiniNBP-5 binds IGF-I with 4-5 higher affinity than NBP-4. Mutations of Leu74 in miniNBP-5 to the BP-4 Met weaken the IGF binding 4-fold, whereas mutation Leu61 to the BP-4 equivalent Tyr has no effect.

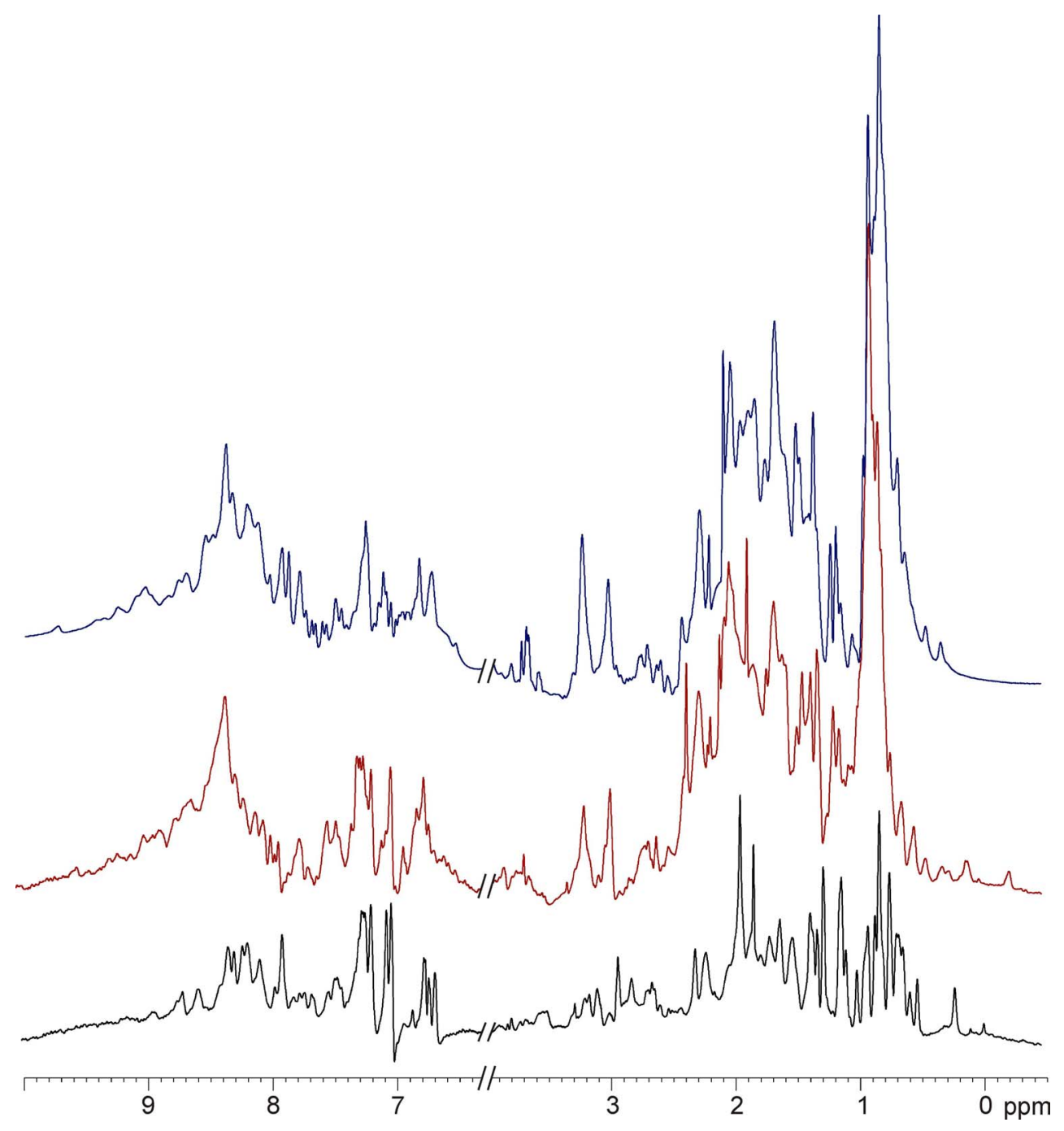


Figure 23. NMR-monitored IGF-I binding by full length IGFBP-4. Diagnostic regions of the 1DNMR spectra of IGFBP-4 (top), IGFBP-4/IGF-I complex (middle) and IGF-I (bottom).

\subsubsection{Inhibition of the IGF-I receptor binding and action by IGFBP fragments}

Effects of binding between IGF-I and IGFBP fragments on the IGF-IR activities were evaluated by measuring the influence of these complex formations on IGF-mediated stimulation of the IGF-I receptor autophosphorylation (Table 8; experimental data in a graph form can be found in Appendix 3). A full-length IGFBP-4 inhibits receptor autophosphorylation with $\mathrm{IC}_{50}$ of about $2.4 \mathrm{nM}$; a $10 \mathrm{kDa}$ NBP-5 fragment with $\mathrm{IC}_{50}$ of about $400 \mathrm{nM}$. The CBP-4 fragment alone showed no significant inhibition up to $1000 \mathrm{nM}$, whereas NBP-4(3-82) started to inhibit at $1000 \mathrm{nM}$. Combination of N- and C-terminal BP-4 domains strongly enhanced inhibition with NBP-4(3-82)/CBP-4(151-232) having $\mathrm{IC}_{50}$ about $700 \mathrm{nM}$; in contrast, complexation of miniNBP-4(39-82)/CBP-4(151-232) showed no inhibition. We have also produced NBP-4(1-92) to check whether the Nterminal extension increases inhibitory activity of the BP-4, as suggested by structural analysis of the IGF-I/NBP-4(3-82) complex. NBP-4(1-92) alone and in combination with CBP-4 showed a 3 fold stronger inhibitions compared to NBP-4(3-82) or NBP-4(382)/CBP-4. This was expected as NBP-4(1-92) exhibits a 2-3 fold tighter binding to IGF-I.

Table 8. IGF-IR inhibitory activities of IGFBP fragments

\begin{tabular}{ll}
\hline IGFBP fragment(s) & $I_{50}\left[\mathrm{nM}^{\mathrm{a}}\right.$ \\
\hline IGFBP-4 & 2.4 \\
NBP-4 (3-82) & 5598 \\
NBP-4 (1-92) & 1960 \\
miniNBP-4 & n.i \\
CBP-4 & n.i \\
NBP-5(1-94) & 444 \\
miniNBP-4 + CBP-4(151-232) & n.i \\
NBP-4(3-82) + CBP-4(151-232) & 767 \\
NBP-4 (1-92)+ CBP-4(151-232) & 290 \\
\hline a Values of IC 50 are the averages of two measurements with accuracy \\
$\pm 10 \%$ n.i., no inhibition.
\end{tabular}




\subsubsection{Fine-tuning of the $\mathrm{N}$-terminal "thumb" residues}

The very first amino terminal residues of IGFBPs have been neglected to date in mutagenesis studies aiming at delineation of the proteins' structure/function relationships (reviewed in Clemmons, 2001). To assess contribution of the "thumb" region of IGFBP to its IGF-I binding activity, a number of N-terminal mutants of NBP-4 was produced and their IGF-I binding activity was compared using isothermal titration calorimetry (Table 9). Differences in $K_{D}$ values among the constructs, although not large, show that extra contacts made by the "thumb" residues present in our constructs do increase the strength of IGF-I binding.

Table 9. List of NBP-4 constructs, their N-terminal sequences and IGF-I binding constants

\begin{tabular}{lrrrcc}
\hline \multicolumn{1}{c}{ Construct } & \multicolumn{3}{c}{ N-terminal sequence } & $\mathrm{K}_{\mathrm{D}}$ [nM] \\
\hline NBP-4(1-82) & & D E A I H C & $317 \pm 19$ \\
NBP-4(3-82) & & A I H C & $865 \pm 78$ \\
NBP-4(6-82) & & & G C & $3350 \pm 590$ \\
BP1-NBP-4 & G A S S G G I G P V V H C & $268 \pm 14$ \\
BP3-NBP4 & G A G A V G I G P V V R C & $316 \pm 27$ \\
ConsBP3-NBP4 & & A P W H C & $544 \pm 45$ \\
& & & \\
\hline
\end{tabular}

\subsubsection{Characterization of the thumb microenvironment in the IGFBP/IGF-I complex by fluorescence spectroscopy}

The construct BP1-NBP-4, with the N-terminal residues Ala, lle and Trp mimicking the thumb region of IGFBP-1, has a tryptophan residue within the thumb and thus can be used to monitor changes in its microenvironment related to the complex formation with IGF-I and CBP-4.

The fluorescence spectrum of Trp3 in BP1-NBP-4, with its maximum at $345 \mathrm{~nm}$ and width of about $57 \mathrm{~nm}$, falls between spectral classes II and III (the classification of tryptophan emission spectra according to Burstain is presented in section 3.4, pp. 3334). Trp3 can be therefore categorized as a solvent-exposed residue, in contact with bound as well as free water molecules. Binding of IGF does not change spectral characteristics of Trp3 (Figure 24 A, B). A slight increase in fluorescence intensity can 
be explained either by resonance energy transfer from IGF tyrosine residues or by partial shielding of the indole from water molecules.

Trp206 of CBP-4, with its emission peak at $340 \mathrm{~nm}$, shows properties of an indole moiety at the protein surface. The observed low intensity of fluorescence emission can be due to quenching by side chains of amino acid residues close to Trp206. Sulfur atoms of disulfide bonds are known to be effective quenchers of tryptophan fluorescence. Cys205 and Cys207 are the closest neighbors of the Trp206. However, as the side chains of two neighboring residues are oriented in opposite directions from the backbone, the distances between the sulfur atoms of disulfide bond Cys205Cys194 and Cys207-Cys228 and the indole ring of Trp206 are too large to influence tryptophan fluorescence $(>5 \AA)$. Other potential Trp206 fluorescence quenchers are Q139 and His141.

Binding of CBP-4 to the NBP4(1-82)/IGF-I binary complex shifts the maximum of Trp206 emission ca. $5 \mathrm{~nm}$ into the shorter wave length and further quenching can be observed (Figure $24 \mathrm{C}$ ). We therefore assume that contribution of the C-terminal Trp206 to the fluorescence of the BP1-NBP-4/CBP-4/IGF-I ternary complex is negligible due to significant quenching of its fluorescence observed in the NBP4(182)/CBP-4/IGF-I complex. The fluorescence spectrum of BP1-NBP-4/CBP-4/IGF-I has nevertheless been corrected for the Trp206 fluorescence in the NBP4(1-82)/CBP4/IGF-I ternary complex.

Drastic changes in the emission properties of Trp3 are observed when CBP-4 joins the BP1-NBP4/IGF-I binary complex. There is a 3-fold increase in the fluorescence intensity accompanied by a $15 \mathrm{~nm}$ blue shift of the peak maximum. The spectrum becomes a model class I spectrum, with maximum at $330 \mathrm{~nm}$ and a width of $50 \mathrm{~nm}$ (Figure $24 \mathrm{~A}, \mathrm{~B}$, Figure 25), and thus is typical for a buried residue. Since fluorescence of tryptophan is susceptible to quenching by water that leads to the decrease in quantum yield when tryptophan becomes exposed to an aqueous environment, this increase in fluorescence of Trp3 upon the CBP-4 binding can be solely attributed to a "water-free" microenvironment. 


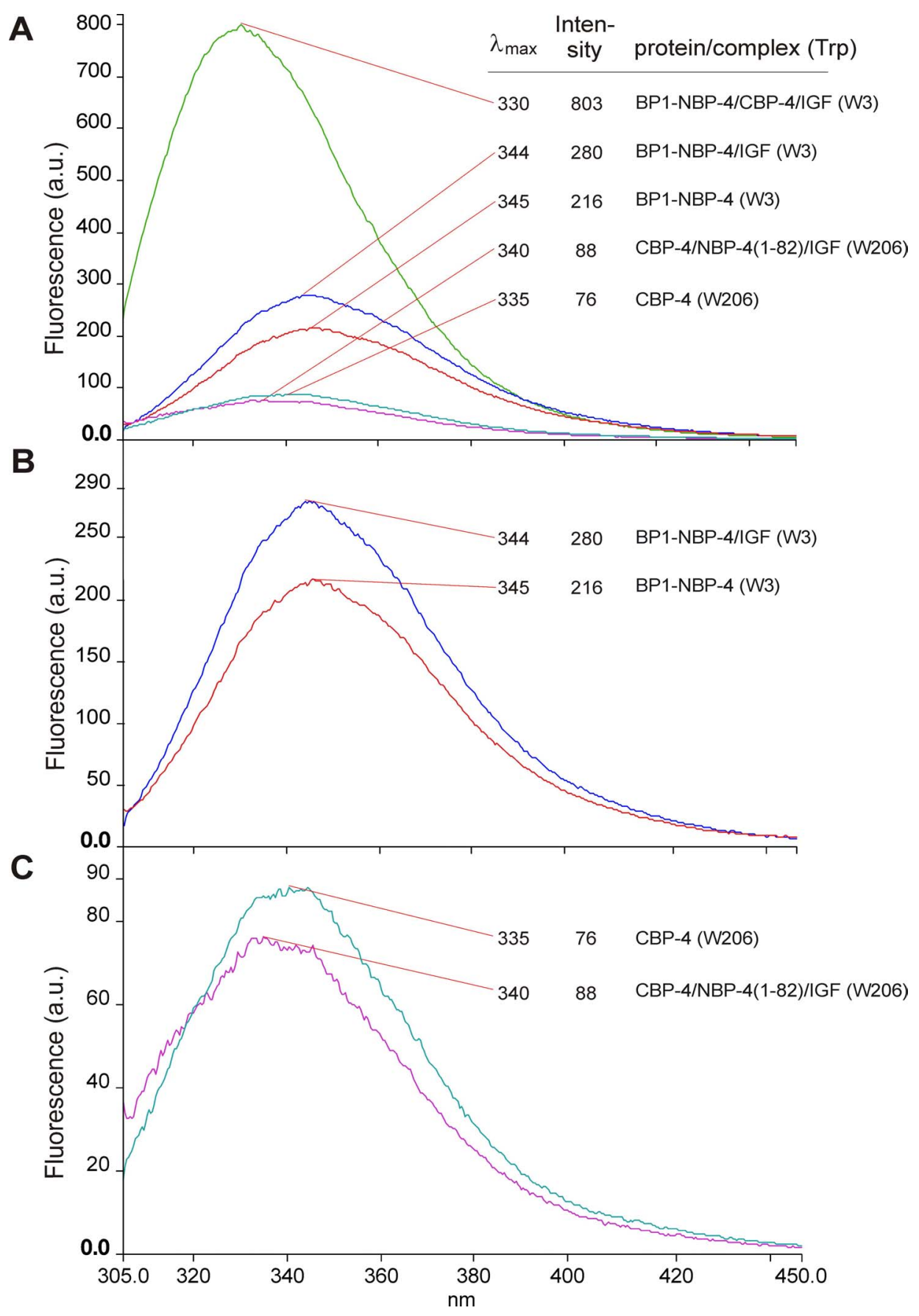

Figure 24. Effects of the binary and ternary complex formations on the fluorescence emission of the N-terminal Trp3 and the C-terminal Trp206. A: tryptophan fluorescence spectra (shown to scale) of BP1-NBP-4 and CBP-4; the free and binary (BP1-NBP-4/IGF-I) and ternary (BP1NBP-4/CBP-4/IGF-I or NBP-4(1-92)/CBP-4/IGF-I) complexes. Emission spectra of the free and IGF-I-bound BP1-NBP-4 (panel B) and CBP-4 in the NBP-4(1-92)/CBP-4/IGF-I complex (panel C) are shown separately for details. The final concentration of all proteins was $4 \mu \mathrm{M}$. The excitation wavelength was adjusted to $295 \mathrm{~nm}$ to minimize Tyr fluorescence contribution and emission was scanned over the range of $305-500 \mathrm{~nm}$. All spectra were corrected for buffer fluorescence; the BP1-NBP-4/CBP-4/IGF-I spectrum was corrected for the Trp206 emission in the NBP-4(1-92)/CBP-4/IGF-I complex. 


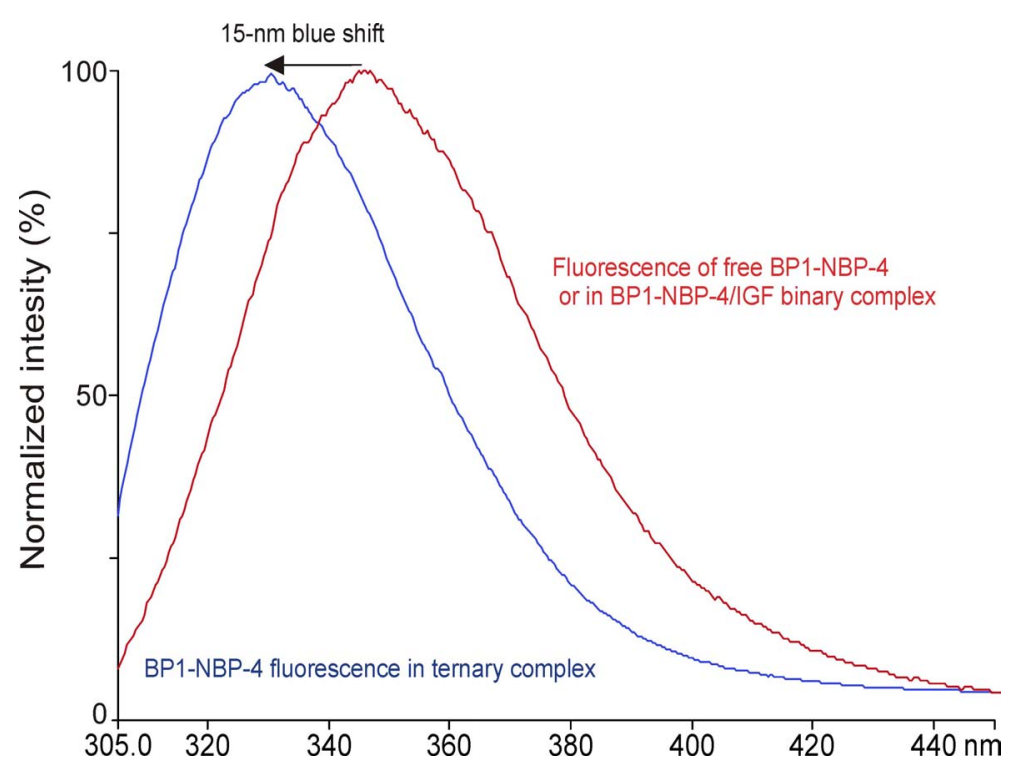

Figure 25. Trp3 of BP1-NBP-4 is buried in a water-free environment upon the CBP-4 binding. Normalized emission spectra of Trp3 in free or IGF-I-bound BP1-NBP-4 (red curve) and in the BP1-NBP-4/CBP-4/IGF-I ternary complex (blue curve).

\subsection{Isoquinoline compound NBI-31772 does not cause dissociation of the IGF-I/IGFBP complex}

Recently Chen and coworkers reported discovery of an isoquinoline-based family of potent inhibitors of the IGF-IGFBP binding, effective in a nM concentrations (Liu et al, 2001, Chen et al, 2001, Zhu et al, 2003). We thought to use an NMR-monitored titration with one of their small molecule compounds to map the binding site of this compound on ${ }^{15} \mathrm{~N}-$ miniNBP-5. We have chosen compound NBI-31772 (1-(3,4dihydroxybenzoyl)-3-hydroxycarbonyl-6, 7-dihydroxyisoquinoline) characterized by the highest IGFBP-3 binding affinity $\left(\mathrm{K}_{\mathrm{i}}=5.6 \pm 0.5 \mathrm{nM}\right.$, as determined by radioactive IGF-I displacement, Chen et al., 2001).

The ${ }^{15} \mathrm{~N}$-miniBP-5 titration with this compound was carried out with molar ratio of the protein to NBI-31772 ranging, respectively, from 1:0.1 to 1:10. No interaction with miniNBP-5 was observed, although the compound was present in a high molar excess. The possibility that isoquinolines disrupt IGF/IGFBP complex through binding with IGF was than investigated. The ${ }^{15} \mathrm{~N}-$ miniBP-5/IGF-I complex was titrated with NBI-31772 in a similar manner, but no changes in the ${ }^{15} \mathrm{~N}-\mathrm{HSQC}$ spectrum were observed, indicating no efficacy of this compound in this assay (Figure 26). Taken together, our results 
show unequivocally lack of interactions of NBI-31772 with IGFBP-5(40-92), miniBP5/IGF-I, or IGF-I. Even weak milimolar associations could be excluded. In addition, we have shown that NBI-31772 cannot dissociate NBP/IGF-I complexes.

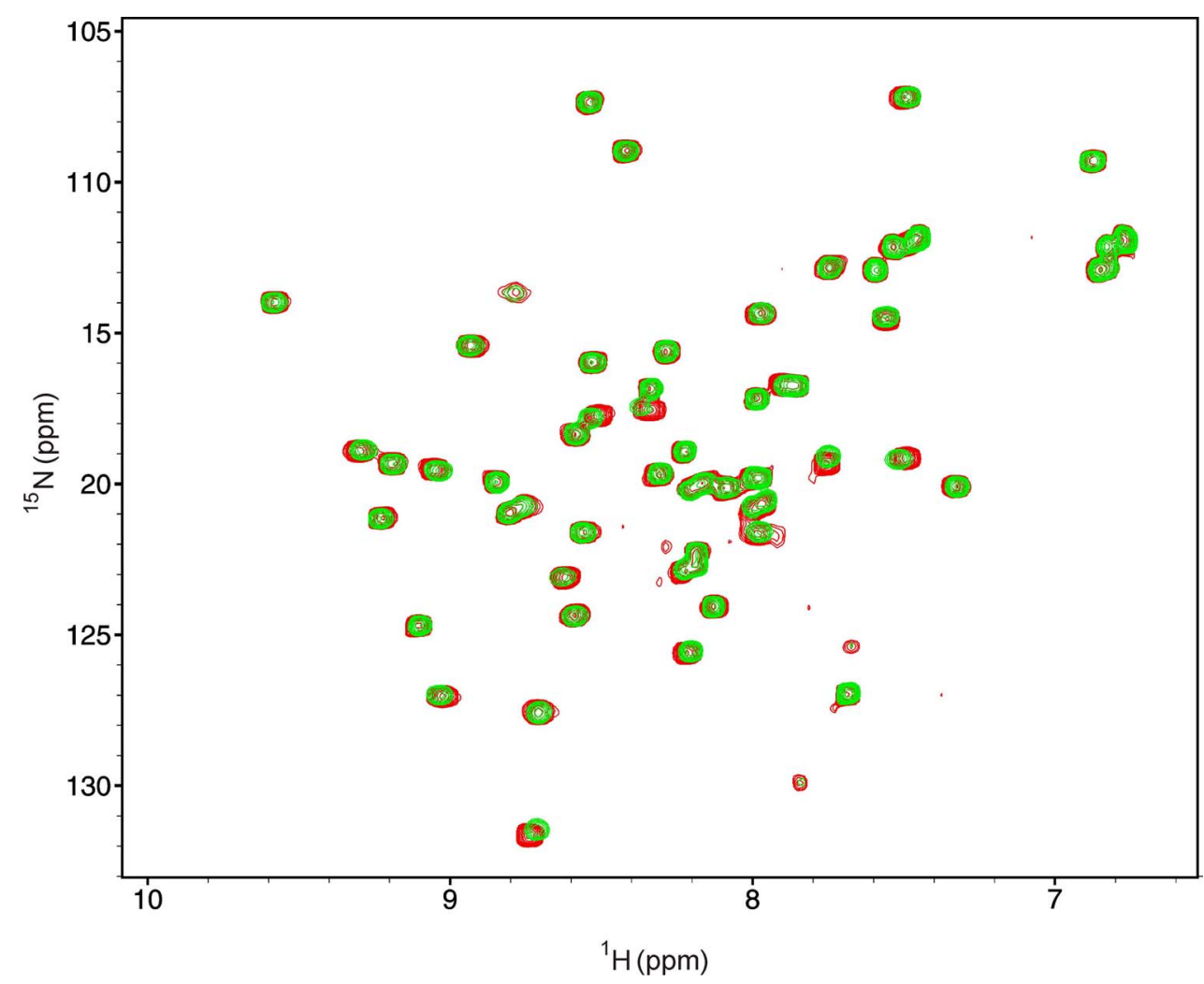

Figure 26. NMR-monitored titration of ${ }^{15} \mathrm{~N}-$ miniNBP-5 with NBI-31772. Superimposed ${ }^{15} \mathrm{~N}-$ HSQC spectra of miniNBP-5 before (red) and after the last step of titration with the isoquinoline compound (green). 


\section{Discussion}

\subsection{Domain organization of IGFBPs}

The molecular size of the six IGFBPs ranges from 22 to $32 \mathrm{kDa}$. The amino- and carboxyl-terminal thirds of the IGFBPs are homologous globular domains, heavily cross-linked by disulfide bonds whose positions are conserved (with an exception of IGFBP-6, missing 6th and 7th N-terminal cysteines). Both $\mathrm{N}$ and $\mathrm{C}$ domains contain IGF binding determinants common for all members of IGFBP family. On the basis of 1D NMR spectra of the intact IGFBP-4 and -5 and their $\mathrm{N}$ - and C-domains we can conclude that their whole central variable domain is disordered, while the first ca. 90 amino acids from the $\mathrm{N}$-terminus form a folded structure. The spectra of the $\mathrm{C}$-terminal fragments indicate presence of disordered regions also within the carboxyl-domains. Comparison of 1D NMR spectra of the $E$. coli expressed full-length recombinant IGFBPs with that of IGFBP-3 produced in Chinese hamster ovary ( $\mathrm{CHO}$ ) cell cultures shows no noticeable differences in the folding extent, proving that the in vitro refolding recreates protein structures seen in the one from the mammalian expression system. Addition of IGF and formation of binary complex has no influence on the folding extent either in the linker region or in the C-domain alone.

\subsection{Contributions of the $\mathrm{N}$ - and $\mathrm{C}$-terminal domains to IGF-I binding}

It is now generally accepted that both $\mathrm{N}$ - and $\mathrm{C}$-domains of IGFBPs are involved in binding to IGFs (Clemmons, 2001; Imai et al., 2000; Shand et al., 2003; Payet et al., 2003). A comprehensive review of the literature on this subject has recently been published by Heady at al. (2004c) in connection with their study of binding of the IGFBP-6 domains to IGFs. This current compilation of the data can be summarized as follows: In general, the strongest binding to IGFs is shown by amino terminal fragments, which however bind to IGF with 10-1000-fold lower affinity than full-length IGFBPs (Andress et al., 1993; Carrick et al., 2001; Cheung et al., 1994; Firth et al., 1998; Galanis et al., 2001; Hashimoto et al., 1997; Kalus et al., 1998; Qin et al., 1998; Standker et al., 2000; Twigg et al., 2000 Imai et al. 2000; Zeslawski et al. 2001).

There are conflicting reports in the literature regarding the role of the C-terminal domains in binding to IGFs. Deletion (Forbes et al., 1998) or point mutation (Bramani 
et al., 1999) of C-domains of IGFBPs clearly leads to lower affinities for IGFs, showing that $C$ domains are involved in binding. However, Galanis et al. (2001) and Carrick et al. (2001) reported that C-domains of IGFBP-2 and IGFBP-3, respectively, bound IGFs with higher affinities than N-domains whereas Vorwerk et al. (2002) reported identical binding affinities for the $\mathrm{N}$ - and C-domains of IGFBP-3. In several studies evidence for such binary interactions were not found, for example, C-terminal fragments of IGFBP-3 (Lalou et al., 1996), IGFBP-4 (Qin et al., 1998) and IGFBP-5 (Kalus et al., 1998) were found not to bind to IGFs.

Many reported data fall in between these two extremes. C-terminal fragments of IGFBP-2 and IGFBP-3 were found to bind IGFs 10-fold and 3 to 1000-fold weaker than full-length IGFBP-2 (Carrick et al., 2001; Ho and Baxter 1997; Wang et al., 1988) and full-length IGFBP-3, respectively (Galanis et al., 2001; Payet et al., 2003; Vorwerk et al., 2002). C-domains of IGFBP-4 (Ständker et al., 2000) and IGFBP-6 (Headey et al., 2004c) were found to bind to IGF-II in a low $\mu$ M range, weaker than a double-digit $\mathrm{nM}$ binding of N-terminal domains. The binding of C-domains to IGF-I was even weaker compared to IGF-II and could not be determined for IGFBP-6 (Headey et al., 2004c). For the IGFBP-4 fragment 136-237 it was estimated to be $>60 \mathrm{nM}$ by plasmon resonance spectroscopy, ligand blot analysis, and saturation and displacement methods (Ständker et al., 2000). Our ITC measurements showed no binding of CBP-4 to either IGF-I or to NBP-4. We next used NMR to check for very weak (i.e. millimolar) interactions (Rehm et al., 2002); standard molecular biology methods for protein binding, such as for example ELISA, RIA, or Biacore fail to detect ligands with weak (i.e. millimolar) affinities. NMR demonstrated lack of even weak binding between CBP4 and IGF-I or CBP-4 and NBP-4.

NBP-4 binds IGF-I with 4-5 fold higher affinity than miniNBP-4. The extra contact sites observed between IGF and NBP-4, in particular involving the two N-terminal hydrophobic residues, may explain this increased affinity. These residues, in BP-4 Ala3 and Ile4, have their counterparts among all 6 IGF binding proteins. There are always 2 nonpolar or aromatic side chain residues at corresponding positions in all IGFBPs. This homology at the extreme $\mathrm{N}$-terminus has been largely overlooked, and apparently has not been considered to be involved in any kind of interaction. These residues belong to the "thumb" fragment in our model of the IGFBP domain architecture. 
The presence of CBP-4 does not influence the parameters of miniNBP-5 binding to IGF, but it does increase NBP-4 affinity to IGF-I, resulting in 3-6 times tighter binding. An increased contact area between NBP-4 and the CBP-4/IGF pair in the ternary complex compared to the NBP-4/IGF complex probably accounts for this, but additional allosteric effects of CBP-4 on NBP-4 or IGF cannot be excluded. The allosteric effects, if they exist, must be small, as shown by the crystal structure data of the ternary complex, well defined for the NBP-4 and IGF-I part of the model.

From the existing X-ray model of the NBP-4/CBP4/IGF ternary complex, we may deduce that the ternary complex formation kinetics are best described by one site model, the binding is probably cooperative and fast.

\subsection{The IGF-I/NBP-4/CBP-4 interaction}

An average length of an N-terminal domain of IGFBPs located upstream from the miniNBP-5-like segment is 43 residues, excluding insertions present in IGFBP-2, and IGFBP-6. This subdomain contains eight cysteines that are strongly conserved across five of the six IGFBPs; the exception is IGFBP-6, which lacks two of the 8 cysteines. To date, only connectivities of the six N-terminal cysteines of IGFBP-6 have been successfully mapped (Neumann et al., 1999). In IGFBP.4, amino-terminal disulfide connectivities of cysteines 17, 23, 35 and 38 suggested by Chelius et al. (2001) using conventional biochemical methods were, according to our structure, assigned incorrectly. Figure 27 depicts the disulphide bridge arrangement in NBP-4(3-38).

The primary IGF-I binding site of NBP-4 is a replication of the binding site found in miniNBP-5. The key IGF binding residues of miniNBP-5 (Gly48, Val49, Tyr50, Arg53, Arg59, Cys60, Leu61, Leu70, and Leu73) are matched by corresponding residues in the C-terminal segment of NBP-4 (e.g. miniNBP-4(39-82)); i.e. Gly47, Val48, Tyr49, Arg52, Arg58, Cys59, Tyr60, Leu69, Leu72).

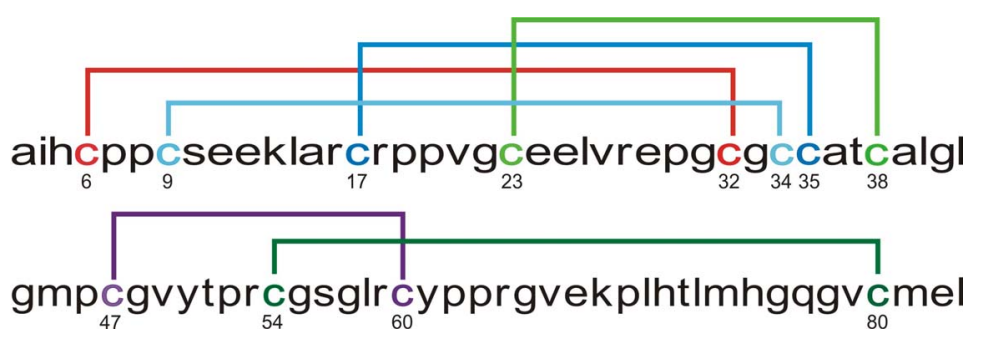

Figure 27. Arrangement of the disulfide bridges of NBP-4. 

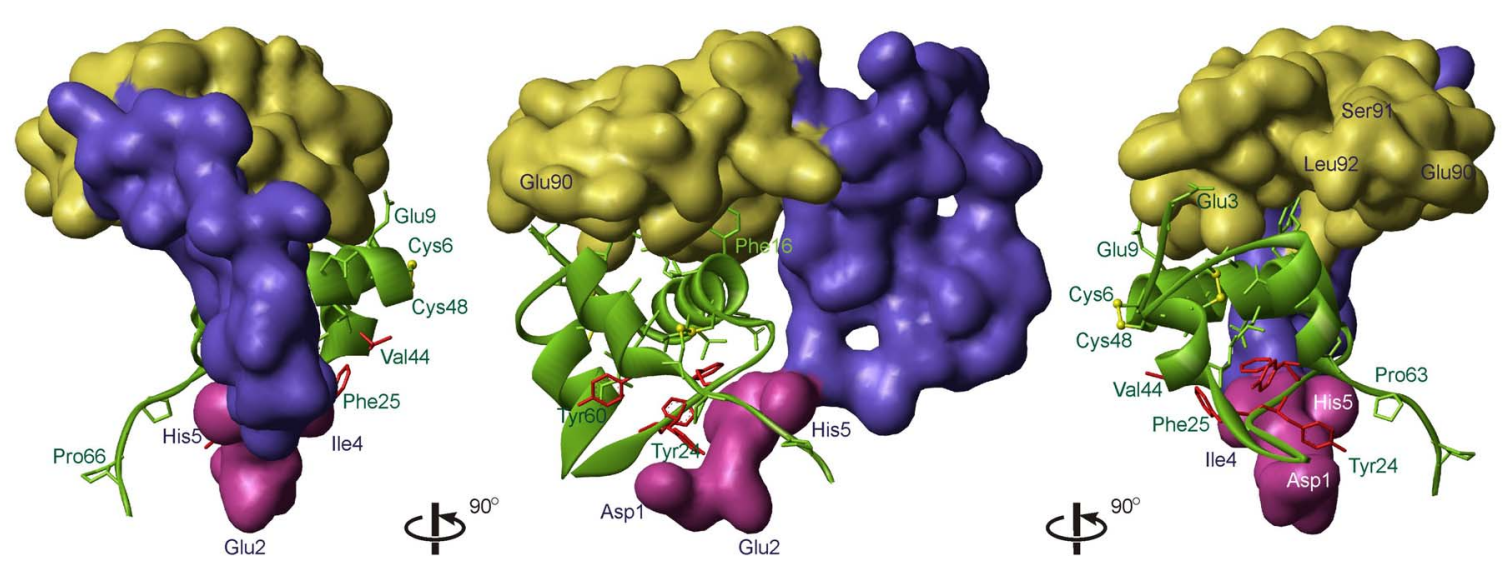

Figure 28. Bipolar binding of IGF-I by NBP-4(1-92). Three views of the binary complex; NBP$4(1-92)$ is presented as a surface: the "base" region is colored in yellow, "palm" in blue and "thumb" in magenta. IGF-I is shown as a green ribbon.

The amino-terminal part of IGFBP-4 can be further divided into two subdomains: the fragment from residues Asp1-Cys39 and the segment Met44-Glu90, joined by a short, glycine - leucine rich linker. Each of the subdomains has its own system of disulfide bridges reinforcing their folds. Evident from the binary complex structure lack of a extensive contact areas between the subdomains that would impose rigid linkers as well as amino acid composition of the linker, prompted us to examine molecular motions in the uncomplexed NBP-4 using NMR ${ }^{15} \mathrm{~N}$ relaxation measurements. The experiment demonstrated that the linker is rigid even in the absence of bound IGF-I. We should mention here that in all structures of either binary complex of IGF-I and NBP-4(3-82) or IGF-I and NBP-4(1-90), as well as in the ternary complex of IGF-I, NBP-4(3-82) and CBP-4 (151-232), the relative orientation of both subdomains is identical.

The N-terminal domain can be viewed as consisting of a globular base, corresponding to the miniBP- 5 that contains the primary IGF binding site, and an extended palm followed by a short hydrophobic thumb (Ala3, lle4). The thumb interacts with IGF residues Phe23, Tyr24 and Phe25 upon complex formation. The palm is rigid because of four disulphide bonds arranged in a ladder-like manner plane. A number of $\mathrm{H}$-bonding inter-subdomain interactions prevent the palm to notably move in respect to the base. 


\section{IGFBP-1}

Homo sapiens

Rattus norvegicus

Mus musculus

Spermophilus

Bos taurus

Danio rerio

\section{IGFBP-2}

Homo sapiens

Rattus norvegicus

Mus musculus

Sus scrofa

Bos taurus

Ovis aries

Gallus gallus

Danio rerio

Sparus aurata

oncorhynchus tshawytscha
A $P$ W $Q$

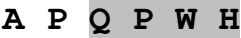

$\begin{array}{lllllllllllllll}A & P & Q & P & W & H\end{array}$

Q $\quad P \quad W \quad H$

$S$ I $Q$ P $\mathrm{L}$ H

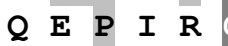

\section{IGFBP-3}

Homo sapiens
Rattus norvegicus
Mus musculus
Sus scrofa
Bos taurus
Danio rerio
consensus

IGFBP-4

Homo sapiens

Rattus norvegicus

Mus musculus

Ovis aries

Sus scrofa

Bos taurus

Gallus gallus

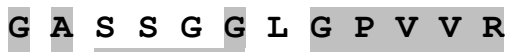

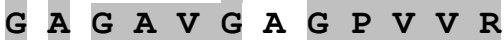

A A G A V G - G P V V R

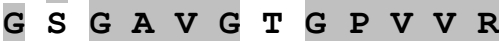

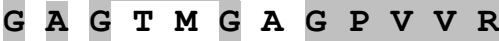

E $\quad S \quad V \quad S \quad P \quad V \quad V \quad R$

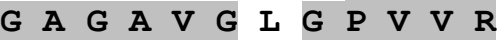

IGFBP-5

Homo sapiens

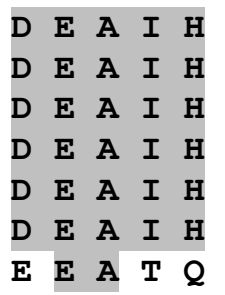

(gilthead seabream)

(Chinook salmon)

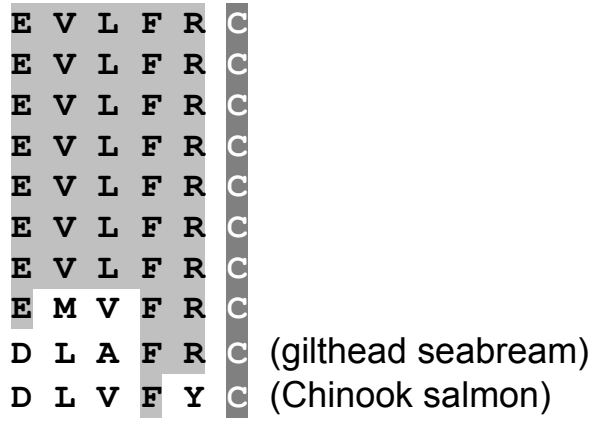

Rattus norvegicus

L $G \quad S \quad F \quad V \quad H$

Mus musculus

Sus scrofa

Gallus gallus

Danio rerio

L G S F V H

L $G \quad S \quad F \quad V \quad H$

L $G \quad S \quad F \quad V \quad H$

L $\quad G \quad S \quad F \quad V \quad$ Q

G $S$ F V P

\section{IGFBP-6}

Homo sapiens

Rattus norvegicus

Mus musculus
A I A R
A I A G
A I A G

Figure 29. Alignment of all available primary "thumb" sequences of IGFBPs from various species. Residues that are identical or conserved are shaded. 
Truncation of the thumb reduces the IGF-I binding of NBP-4 to that of miniNBP-4 (residues 39-82), suggesting that the palm does not contribute directly to IGF binding. The segment appears therefore to serve solely a mechanical purpose of a rigid linker between primary binding sites - the base and the thumb residues. The central element of the palm has a GCGCCXXC consensus motif, around which the polypeptide chain (residues Cys6-Cys23) is bent forming a disulfide ladder and assuring proper spatial relationship between the base and the thumb.

Substitution of the IGFBP-4 thumb with a corresponding region from two other IGFBPs does not influence the strength of IGF-I binding markedly. Interestingly, available sequences of IGFBPs from different species show a remarkable high degree inter-species conservation of thumb residues within a one type of IGFBP (Figure 29), implying that the sequence of a thumb may confer unique properties to each IGFBP.

The experiment utilizing Trp3 of BP1-NBP-4 as a fluorescence probe shows that upon C-terminal domain binding to the NBP-4/IGF-I binary complex, microenvironment of the tryptophan changes its character. Formation of the IGFBP/IGF-I complex leads to enclosure of the thumb residues into a rigid, water free core. To exclude water from the Trp3 microenvironment, the interacting molecules must show high degree of surface complementarity in the immediate vicinity of the thumb. The experiments show that $\mathrm{N}$ - and C-terminal domains of IGFBP cooperate to block the primary IGF-IR binding site of IGF-I: the thumb hinders the access to the Phe23-Tyr24-Phe25 pocket of IGF-I, while the C-terminal domain reinforces further this hindrance in agreement with our NBP-4(3-82)/CBP-4/IGF-I ternary complex model.

Bach et al. (1993) in their mutagenesis study on IGFBPs-binding determinants of IGF-II reported that Phe26 of IGF-II is the second most important site in recognition of IGF-II by IGFBPs (after the stretch of amino acids Phe48-Arg49-Ser50). The mutant [Ser26]IGF-II bound IGFBP-1, -4 and -6 with only 1-7\% of the wt-IGF-II affinity. Similar results were obtained by Darren et al. (1996): mutation of the IGF-I residue Phe23 (corresponding to Phe26 in IGF-II) lowered the affinity to all IGFBPs a 100-fold. In contrast, Dubaquie et al. (1999) pointed to the IGF-I residue Phe25 as the pivotal IGFBP binding determinant. Alanine-scanning mutagenesis studies showed that IGF-I with Phe23 replaced by Ala exhibits only slight loss of affinities to IGFBP-1 and -3; relative affinities being 1.3 and 2.9-fold lower than those of wild type protein for IGFBP1 and IGFBP-3, respectively. More significant drop in affinity is observed when Phe25 is mutated to Ala: the affinity of IGFBP-1 and IGFBP-3 for the mutant is 25 and 5 times 
lower than that for the wild type IGF-I, respectively. IGFBP-1 responds to the mutation Phe25Ala noticeably stronger than IGFBP-3. In IGFBP-3, both hydrophobic positions of the thumb consensus sequence are occupied by valines, while in IGFBP-1 there is a proline (the only exception from the consensus in the IGFBPs family) and tryptophan at the respective sites. The bulk of the Trp side chain compensates excessively for lack of a hydrophobic side chain at the neighboring position (van der Waals volume of Pro+Trp is 253, Val+Val, 210). The indole ring of tryptophan can participate in aromatic stacking interactions with neighboring aromatic residues of IGF in the binary complex. Substitution of Phe by Ala does not change the overall hydrophobic environment of the patch, but reduces its aromatic character. Differences in interactions of Phe25 with Cdomains of IGFBPs will also contribute to different behavior among IGFBPs.

Effects of substituting IGF-I Tyr24, and its IGF-II counterpart Tyr27, on IGFBPs binding have also been studied. Mutations of Tyr27 to a hydrophobic residue showed only minor decrease in the IGFBPs binding affinity (Roth, 1991, Bach, 1993). Similarly, mutation of IGF-I Tyr24 to Leu (Baxter et al, 1992) or Ala (Dubaquie, 1999) had a relatively little effect on IGFBPs binding. Substitution of Tyr27 with Glu resulted in a 40fold lower IGFBP-3 binding affinity compared with the wild type IGF-II (Roth, 1991).

The C-domain increases affinity of IGFBP-4 for IGFs. The low resolution structure of the ternary complex of NBP-4(3-82)/IGF-I/CBP-4(151-232) provided the binding site for the C-domain on IGF-I. CBP-4 contacts both the amino-terminal part of NBP-4 and IGF-I. On IGF, a segment Cys6-Val12, which is in an extended conformation, Tyr24, Phe25, GIn26, Val 44, Cys48 and Phe49 are the key interacting residues. Among them Tyr24, Phe25, Val44 are the determinants of IGF binding to its IGF-I receptor.

Headey et al. (2004a) have recently mapped the binding site of the C-domain of IGFBP-6 on IGF-II using NMR. The contact area lies adjacent to and between the binding sites for the IGFBP N-domain and IGF-I receptor and includes residues Thr7, Cys9, Gly10-Thr16, GIn18, Cys21, Phe28, Ile42, Val43, Glu44, Cys46, Cys47, Phe48, Arg49 and Cys51. The binding site defined by the authors agrees with our findings, although their contact surface is slightly larger than the one obtained from our X-ray data: residues of IGF-I corresponding to IGF-II Thr7, Leu10, GIn18, Cys21, Ile42, Glu44, Cys46, Arg49 and Cys51 are too far away from CBP-4 to be directly involved in binding. These differences are however not unexpected as the interfaces for proteinprotein interactions identified by NMR chemical shift perturbation methods are similar but not identical to those revealed by X-ray crystallography, or other NMR methods, 
such as for example, hydrogen/deuterium exchange experiments (Takahashi et al., 2000; Stoll et al., 2001).

The location of C-terminal residues of NBP-4 shown in Figures 18 and 28 suggests the location for the central L-domain of IGFBP-4 in the IGF/IGFBP interaction. The L-domain, which constitutes roughly $1 / 3$ of the amino acid sequence in IGFBPs (28\% in IGFBP-4 and up to $36 \%$ in IGFBP-3), would cover most of the remaining parts of IGF that already were not enclosed by NBP-4 and CBP-4 domains. It appears therefore that IGFs are encapsulated by IGFBPs before their release after IGFBP proteolysis.

\subsection{Inhibition of the IGF-I receptor binding and action by IGFBP fragments}

Site-directed mutagenesis studies of mapping binding sites of IGFs for IGF-IR and the insulin receptor (IR) showed that the major determinants of binding in IGF-I are Tyr60, Tyr24, Phe23, Val44 (Cascieri et al., 1988; Bayne et al., 1990; Hodgson et al., 1996; Sakano et al., 1991; Perdue et al., 1994), and possibly Phe25 and Arg21 (Hodgson et al., 1996; Torres et al., 1995; Cooke et al., 1991) (Figure 30). The most notable feature evident from the earlier structural studies on the miniNBP-5/IGF-I complex was that Phe23-Tyr24-Phe25, Val 44, and Tyr60 form a fully solvent-exposed hydrophobic binding site for IGF-IR that is located on the opposite side of IGF to that used for the binding to mini-IGFBP-5 (Zeslawski et al., 2001). The additional N-terminal subdomain 1-38 of NBP-4 on the other hand reaches the IGF-I Tyr24 and its surroundings primary through the "thumb" segment residues Ala3 and lle4 of NBP-4 that fill a hydrophobic cleft of IGFs' Phe23, Tyr24 and Phe25. We would therefore expect to see some inhibition of activity of IGF-IR for the entire NBP-4 (e.g. which include the "thumb" residues) compared to the miniNBP-4 domain only. This has indeed been confirmed by the data on the inhibition of autophosphorylation of IGF-IR (Table 8).

Both IGF-I and IGF-II bind to the IGF-I receptor with high affinity $\left(K_{D}=1.5\right.$ and 3.0 $\mathrm{nM}$, respectively; Loddick et al., 1998). In our assay, a full-length IGFBP-4 inhibited IGF-IR autophosphorylation with $\mathrm{IC}_{50}$ of about $2.4 \mathrm{nM}$ (Table 8). The N-domains showed some inhibition, with the longer NBP-4 fragment NBP-4(1-92) (and NBP-5(194)) exhibiting superior inhibition relative to that of NBP-4(3-82). This trend parallels stronger IGF binding of these fragments relative to NBP-4(3-82). MiniNBP subdomains showed no inhibition, indicating that the N-terminal "thumb" part of BPs is important for inhibition of IGF action. 
As discussed above, the N-terminal domain of IGFBPs is characterized by high degree of rigidity. This may be of significance when the competition with IGF-IR for IGF binding is concerned. To displace the hydrophobic thumb that covers the primary IGFIR binding site of IGFs (IGF-I, Phe23-Phe25), the receptor has to lift also the rest of the $\mathrm{N}$-terminal domain, which is bound on the opposite side of the IGF-I molecule, and does not prevent receptor binding on its own (Kalus et al. 1998). Thus, the thumb does not have to significantly contribute to the overall binding affinity of IGFBPs for IGFs. This mechanism is expected to be shared by all IGFBPs given the conserved arrangement of the $\mathrm{N}$-terminal cysteine residues and the consensus presence of two hydrophobic residues at positions -2 and -3 with respect to the first $\mathrm{N}$-terminal Cys residue.

The structure of the ternary complex suggests that addition of CBP-4(151-232) to the NBP-4(3-82)/IGF interaction should enhance inhibition of IGF-IR activity, and indeed a 6-fold increase is seen (Table 8). Our data are in agreement with that of Payet et al. (2003) who have recently demonstrated that isolated amino-terminal and carboxy-terminal domains of IGFBP-3, residues 1-88 and 185-264, respectively, cooperate in the presence of IGFs to form high-affinity complexes that retain the ability to block IGF activity. We observe no inhibition for miniNBP-4(39-82) and CBP-4, indicating again a crucial role of the $\mathrm{N}$-terminal "thumb" residues of BPs in blocking IGF activity.

Proteolytic cleavage has gained wide acceptance as the predominant mechanism for IGF release from IGFBPs (Bunn and Fowlkes, 2003). The current paradigm for IGF release proposes that proteolysis takes place at the central L-domain producing low affinity $\mathrm{N}$ - and $\mathrm{C}$-terminal fragments that cannot compete with IGF receptors for IGFs. The data presented by Payet et al. (2003), together with our results, clearly indicate that IGFBP fragments still retain properties to inhibit IGF activity, and therefore this paradigm has to be revised. The structures of the IGF/BP-4 complexes presented here suggest a following model for IGF binding proteins actions (Figure 31): The data indicates that IGFs, which are approximately globular structures, are encapsulated by IGFBPs. The N-terminal domain of IGFBPs plays an important role in binding IGFs. The globular C-terminal part of this domain, miniNBP, serves as a primary docking site for IGFs; the N-terminal subdomain, which consists of a "palm" and a "thumb", reaches a hydrophobic IGF receptor binding site of IGFs with the conserved thumb hydrophobic residues. Next, the C-terminal domain, which covers roughly an equal surface of IGF 
to that of the N-terminal domain, shields the IGF receptor binding site of IGFs, close to the N-terminal thumb fragment residues, thus cooperatively enhancing inhibition of IGF receptor activity. The central domain, which is unstructured and flexible, acts as a "mechanical flap" that covers most of IGF not yet covered by N-terminal and C-terminal domains.
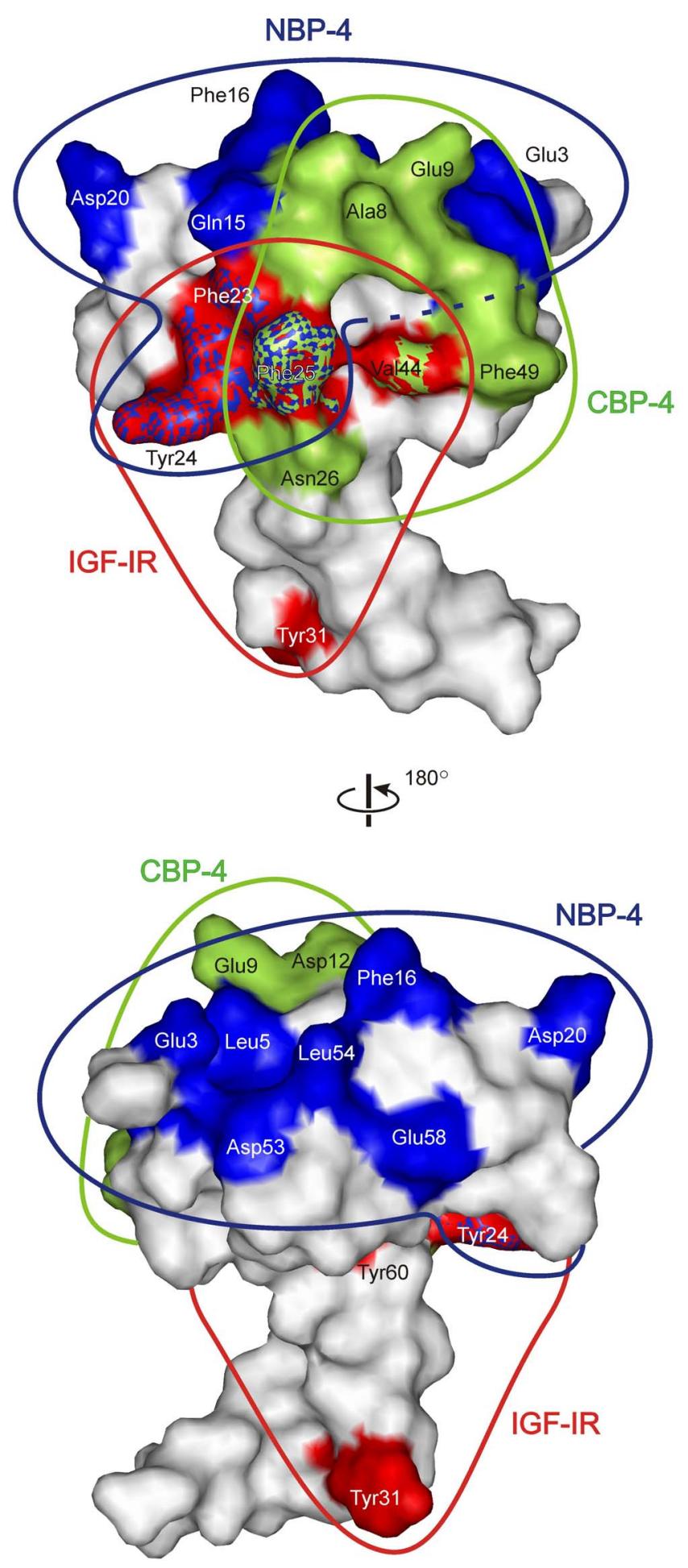

Figure 30. Interaction of IGF-I with IGFBP-4. Schematic of the surface of IGF-I showing the binding site for the IGF-IR (red), $\mathrm{N}$-terminal domain of IGFBP-4 (blue) and CBP-4 (green). 


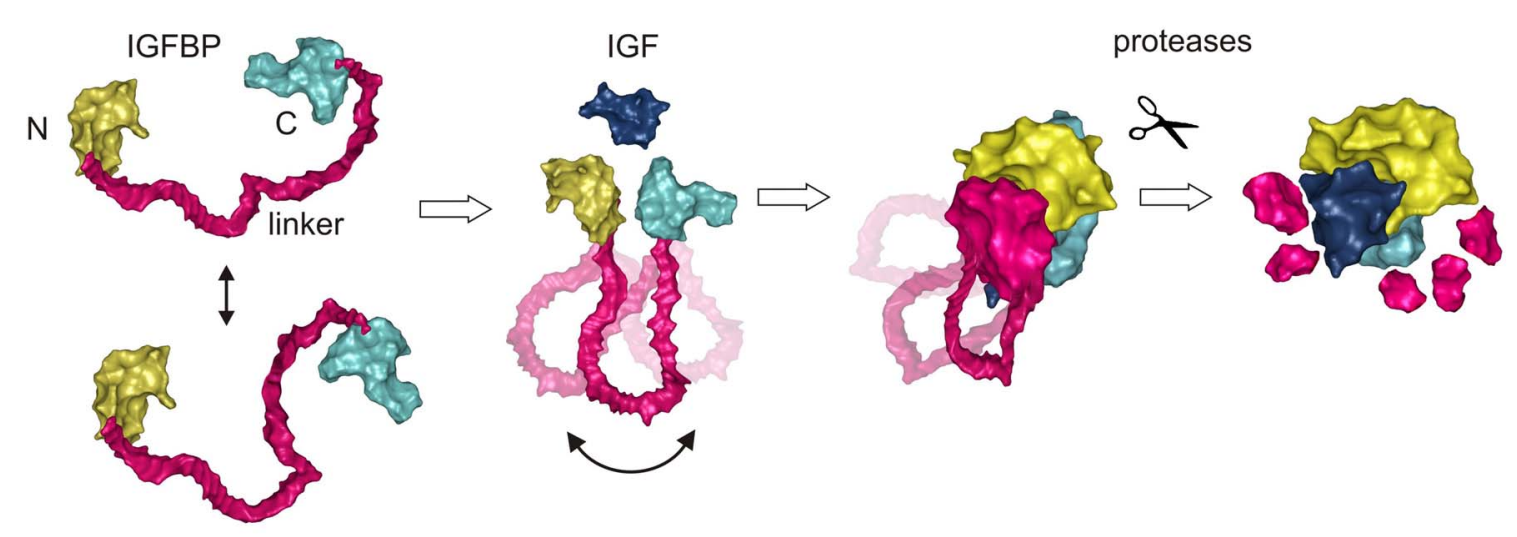

Figure 31. A model of IGFBP/IGF complex and its regulation by proteolysis. IGF is in dark blue, NBP in yellow, and CBP in light blue. The linker domain (in purple) is hypothetical and its flexibility is ephasized. The last step of the reaction depicts proteolysis of IGFBP.

Proteolysis of the IGF/IGFBP capsule would first remove the central domain residues by degradation. This partial removal of the capsule exposes IGF but still maintains IGF inhibition as long as the thumb and/or CBP fragments of IGFBPs are not removed.

Data on structural determinants of IGFBP/IGF binding can be used for design of targets which could regulate the actions of IGFs. This can be carried out either by sitedirected mutagenesis of IGFBPs and thus producing IGFBPs of modified binding affinity, or by design of novel, low molecular weight ligands. For example, IGF-I and IGF-II exhibit neuroprotective effects in several forms of brain injury and neurodegenerative disease. This implies that targeted release of IGF from their binding proteins might have therapeutic value for stroke and other IGF responsive diseases.

Inhibition of IGF-stimulated cancer cell growth via receptor intervention is a major strategy in anticancer drug discovery as shown by the efforts of pharmaceutical companies to develop both protein and small molecule antagonists toward the receptor (LeRoith and Helman, 2004; Ali et al., 2003; Mazerbourg et al., 2004; Firth and Baxter, 2002; Cohen et al., 2000; Khandwala et al., 2000; Bach, 1999). Mutagenesis of the thumb fragment to increase the IGF/BP fragments binding or to enhance their inhibition of IGF-IR action can therefore offer a therapeutic strategy against cancers that are the type I IGF receptor dependent. 


\section{Summary}

Insulin-like growth factor binding proteins (IGFBPs) control bioavailability, activity and distribution of IGF-I and -II in the serum. While IGFBPs have also IGF-independent direct actions, all IGF-dependent actions, positive or negative, i.e. potentiation or inhibition of its mitogenic and metabolic effects, depend on the formation of highaffinity complexes with IGFs. Amino- and carboxyl-terminal fragments of IGFBPs, the two conserved domains of IGFBPs, form together a high-affinity IGF binding site, but their relative contribution to the binding affinity varies among IGFBPs.

The study reported in this thesis employed techniques of nuclear magnetic resonance and X-ray crystallography to gain a more detailed understanding of the IGFBPs domain organization and structural requirements for binding to IGFs. An interand intra-domain arrangement and the extent of folding within domains of IGFBP, -4 , and -5 have been determined with the aid of NMR spectra.

This thesis presents $1.6 \AA$ and $2.5 \AA$ resolution structures of binary complexes between IGF-I and two amino-terminal domain constructs of IGFBP-4 (NBP-4, residues 3-82 and 1-92), and a model of the ternary complex of IGF-I, NBP-4 and the carboxyl-terminal domain (CBP-4, residues 151-232) derived from the $2.1 \AA$ diffraction data. Key features of the structures include: 1) a disulphide bond ladder (the "palm") that binds to IGF through the extreme-N-terminal residues (the "thumb"), which partially mask the IGF residues responsible for type I IGF receptor (IGF-IR) binding; 2) the high affinity IGF-I interaction site formed by residues 39-82 in a globular fold (the "base"), comprising interlaced hydrophobic side chains that protrude from both IGF-I and NBP4 , and the surrounding network of polar interactions, and 3) CBP-4 interactions with both NBP-4 and IGF-I. The mechanical role of the "palm" and importance of the "thumb" residues for IGF binding was confirmed and quantitatively assessed with ITC utilizing a number of mutants and truncated forms of IGFBP-4. A rigid character of the whole $\mathrm{N}$-terminal domain was confirmed by NMR ${ }^{15} \mathrm{~N}$ relaxation measurements. A new insight was gained from these experiments into the involvemet of the IGFBP aminoterminal domain in the inhibition of the IGF-IR activity.

The model of the NBP-4/CBP-4/IGF-I ternary complex explains the relative arrangement of the domains and IGF and shows that the C-terminal domain, CBP-4, 
contacts both the N-terminal domain and IGF-I, although, as determined by gel filtration, ITC and NMR, does not bind individually to either IGF-I or NBP-4. The interaction of CBP-4 and NBP-4 in the ternary complex was also analysed by monitoring Trp3 of the BP1-NBP-4 as a fluorescence probe. The study showed that the Trp3 is burried upon the ternary complex formation in a water free, rigid environment.

Due to the high degree of sequence homology within the investigated domains, the interactions described above can be regarded as prototypes for all members of the IGFBP family. 


\section{Zusammenfassung}

IGFBPs (Insulin-like growth factor binding proteins) kontrollieren die Bioverfügbarkeit, Aktivität und Verteilung von IGF-I und -II im Serum. Während IGFBPs auch IGFunabhängige direkte Wirkungen haben, hängen alle IGF-abhängigen Aktionen, positiv oder negativ, d. h. Potenzierung oder Hemmung der mitogenetischen und metabolischen Effekte, von der Bildung hoch affiner Komplexe mit IGFs ab. Aminound Carboxyterminale Fragmente von IGFBPs, den zwei konservierten Domänen von IGFBPs, bilden zusammen eine hoch affine IGF Bindestelle, aber ihr relativer Beitrag zur Bindungsaffinität variiert unter IGFBPs.

Die in dieser Doktorarbeit beschriebenen Untersuchungen benutzten die Techniken des NMR (Nuclear Magnetic Resonance) und der Röntgenkristallographie um ein vertieftes Verständnis der Domänenorganisation der IGFBPs und der strukturellen Voraussetzungen der Bindung der IGFs zu erwerben. Ein Inter- und IntraDomänen-Arrangement und der Faltungsgrad innerhalb der Domänen von IGFBP-4 und -5 ist mit Hilfe von NMR bestimmt worden.

Diese Doktorarbeit zeigt Strukturen mit 1,6 $\AA$ und 2,5 $\AA$ Auflösung des binären Komplexes zwischen IGF-I und zwei Konstrukten der amino-terminalen Domäne von IGFBP-4 (NBP-4, Aminosäuren 3-82 bzw. 1-92) und ein Modell des ternären Komplexes von IGF-I, NBP-4 und der carboxy-terminalen Domäne (CBP-4, Aminosäuren 151-232) erhalten von Diffraktionsdaten von 2,1 $\AA$ Auflösung. Die wichtigsten Eigenschaften der Strukturen beinhalten: 1) eine Leiter von Disulfidbrücken (die „Handfläche“), die an IGF durch extreme N-terminale Reste (der „Daumen“) bindet, das zum Teil die IGF Reste abdeckt, die für die Typ I IGF Rezeptorbindung (IGF-1R) verantwortlich sind; 2) die hoch affine IGF-I Interaktionsstelle gebildet von Resten 39-82 in einer globulären Faltung (die „Basis“), die verkettete hydrophobe Seitenketten, die aus IGF-I und NBP-4 herausragen, und ein umgebendes Netzwerk von polaren Wechselwirkungen umfasst; und 3) CBP-4 Interaktionen mit sowohl NBP4 als auch IGF-I. Die mechanische Rolle von „Handfläche“ und die Wichtigkeit des „Daumens“ für die IGF Bindung wurde mit ITC bestätigt und quantitativ bestimmt, indem zahlreiche Mutanten und trunkierte Formen von IGFBP-4 verwendet worden sind. Der starre Charakter der gesamten $\mathrm{N}$-terminalen Domäne wurde mit NMR ${ }^{15} \mathrm{~N}$ 
Relaxationsmessungen bestätigt. Ein neuer Einblick in die Beteiligung der Nterminalen Domäne von IGFBP an der Hemmung der IGF-IR Aktivität wurde durch diese Experimente gewonnen.

Das Model des ternären NBP-4/CBP-4/IGF-I Komplexes erklärt das relative Arrangement der Domänen und IGF und zeigt, dass die C-terminale Domäne, CBP-4, sowohl die N-terminale Domäne als auch IGF-I berührt, obwohl sie, wie mit Gelfiltration, ITC und NMR bestimmt, weder an IGF-I noch NBP-4 individuell bindet. Die Interaktion von CBP-4 und NBP-4 im ternären Komplex wurde auch analysiert, indem Trp3 des BP1-NBP-4 als Fluoreszenz Probe überwacht wurde. Die Untersuchung zeigte, dass Trp3 auf die ternäre Komplexbindung hin in einer wasserfreien, starren Umgebung begraben wird.

Aufgrund des hohen Grades der Sequenzhomologie innerhalb der erforschten Domänen können die oben beschriebenen Wechselwirkungen als Prototyp für alle Mitglieder der IGFBP Familie angesehen werden. 


\section{Appendix}

\subsection{Abbreviations and symbols}

-1D

- 2D

- $\AA$

- aa

- ALS

- APS

- bp

- BSA

- cDNA

- COSY

- Da

- DMSO

- DNA

- Dnasel

- EDTA

- ELISA

- $g$

- $\mathrm{GH}$

- GSH

- GSSG

- GST

- HSQC

- $\mathrm{Hz}$

- IGF

- IGFBP

-IGF-IR

- IGF-IIR

- IMAC one-dimensional

two-dimensional

Ångstrøm $\left(10^{-10} \mathrm{~m}\right)$

amino acid

acid labile subunit

ammonium peroxodisulfate

base pair

bovine serum albumin

complimentary DNA

correlation spectroscopy

Dalton (g mol-1)

dimethylsulfoxide

deoxyribonucleic acid

deoxyribonuclease I

ethylenediamine tetraacetic acid

enzyme-linked immunosorbant assay

gravity $\left(9.81 \mathrm{~m} \mathrm{~s}^{-2}\right)$

growth hormone

reduced glutathione

oxidized glutathione

glutathione S-transferase

heteronuclear single quantum coherence

Hertz

insulin-like growth factor

IGF binding protein

IGF receptor type I

IGF receptor type II

immobilized metal affinity chromatography 
- IPTG

- IR

- IRS

- ITC

- $\mathrm{K}_{\mathrm{D}}$

- LB

- MAD

- MAP

- MIR

- MM

- MW

- NiNTA

- NLS

- NMR

- NOE

- NOESY

- OD

- P3K

- PAGE

- PBS

- ppm

- RMSD

- SAR

- SDS

- TB

- TEMED

- TOCSY isopropyl- $\beta$-thiogalactopyranoside

insulin receptor

insulin receptor substrate(s)

isothermal titration calorimetry

dissotation constant

Luria-Broth medium

multiwavelength anomalous diffraction

mitogen-activated protein kinase

multiple isomorphous replacement

minimal medium

molecular weight

nickel-nitrilotriacetic acid

nuclear localization signal

nuclear magnetic resonance

nuclear Overhauser effect

nuclear Overhauser enhancement spectroscopy

optical density

phosphatidylinositol 3-kinase

polyacrylamide gel electrophoresis

phosphate-buffered saline

parts per million

root mean square deviation

structure-activity relationship

sodium dodecyl sulfate

terrific broth

$\mathrm{N}, \mathrm{N}, \mathrm{N}$ ',N'-tetramethylethylendiamine

total correlation spectroscopy

Amino acids and nucleotides are abbreviated according to either one or three letter IUPAC code. 


\subsection{Isothermal titration calorimetry}
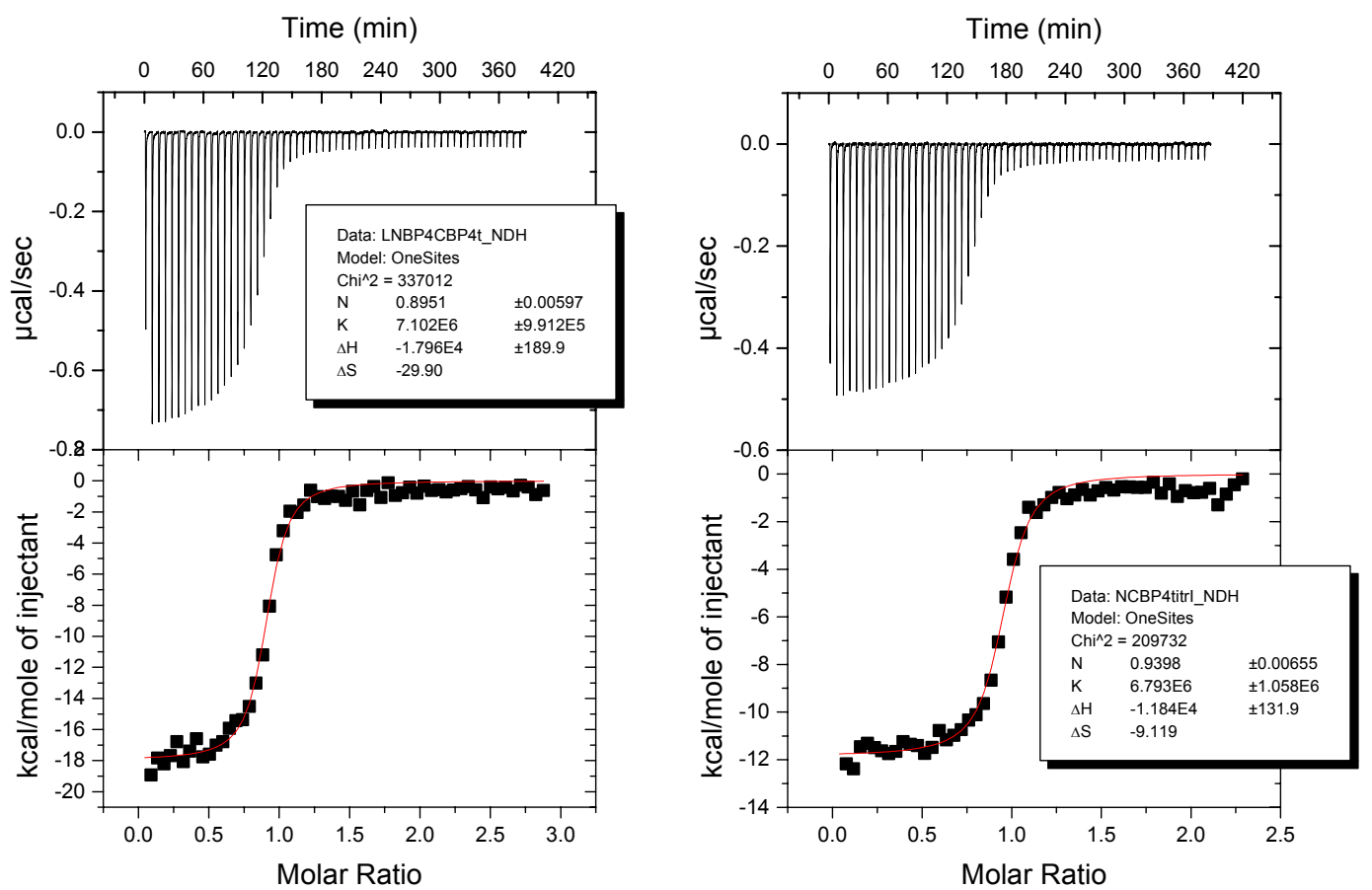

CBP-4+NBP-4(1-92) (1:1) titrated with IGF

CBP-4+NBP-4(3-82) (1:1) titrated with IGF

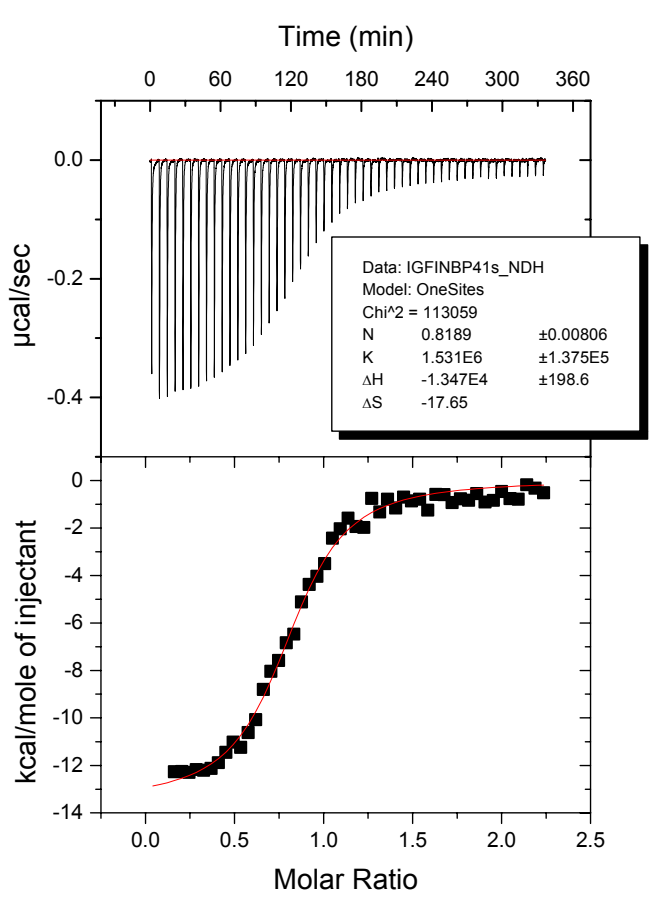

IGF titrated with NBP-4(3-82)

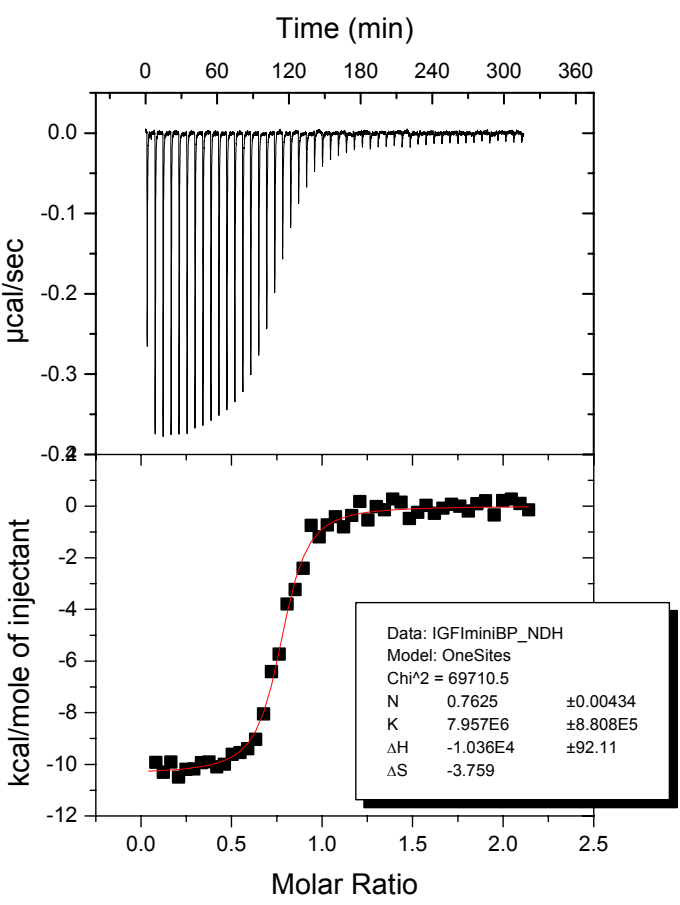

IGF titrated with miniNBP-5 


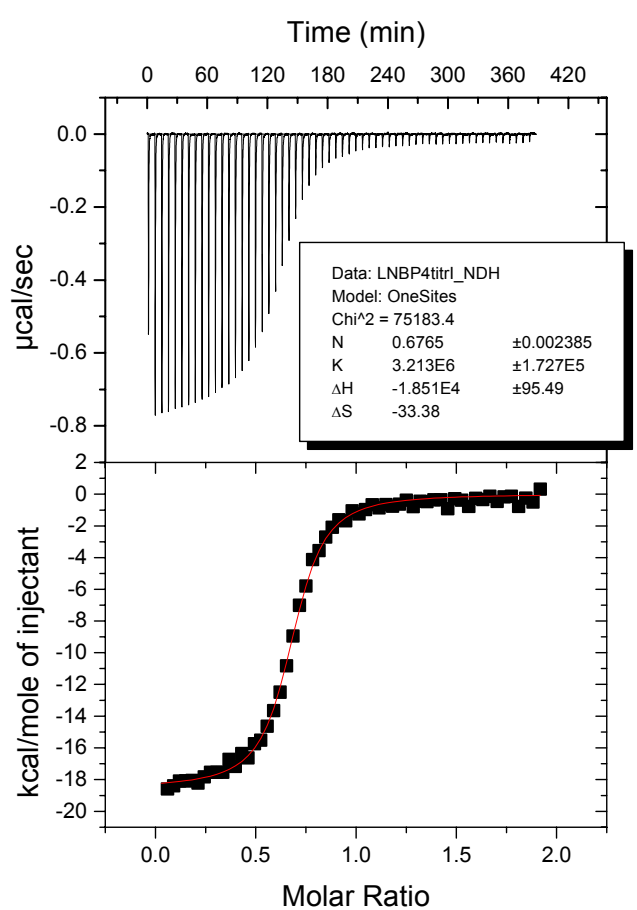

NBP-4(1-92) titrated with IGF

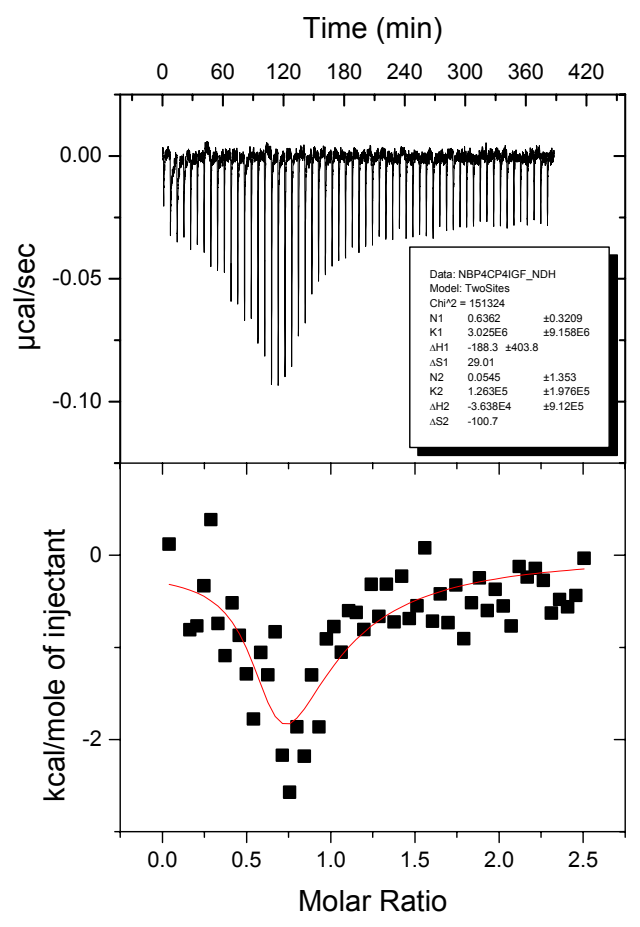

NBP-4(3-82) + IGF titrated with CBP-4

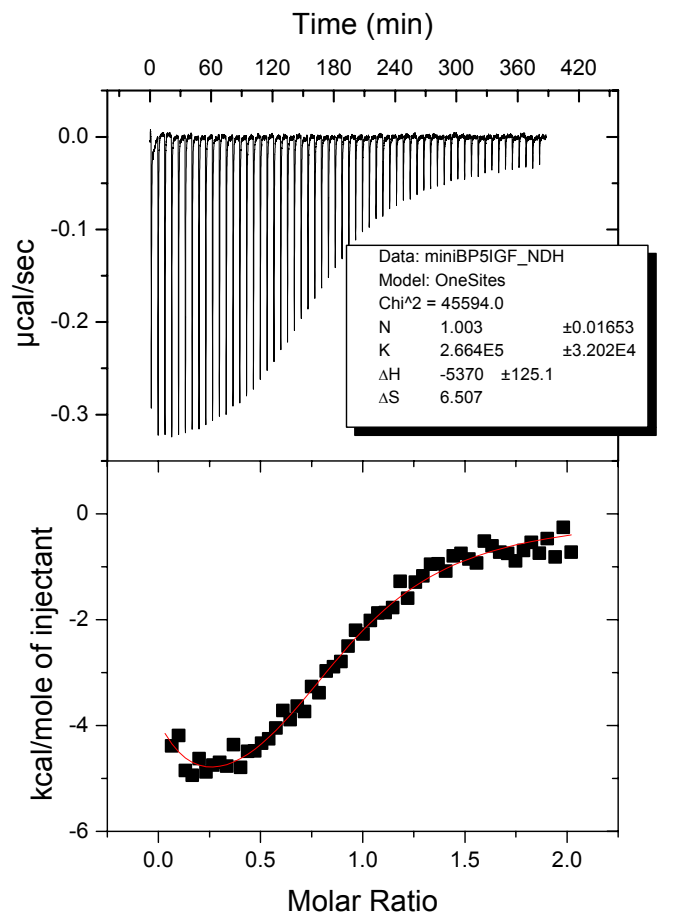

miniNBP-5 + IGF (1:1) titrated with CBP-4

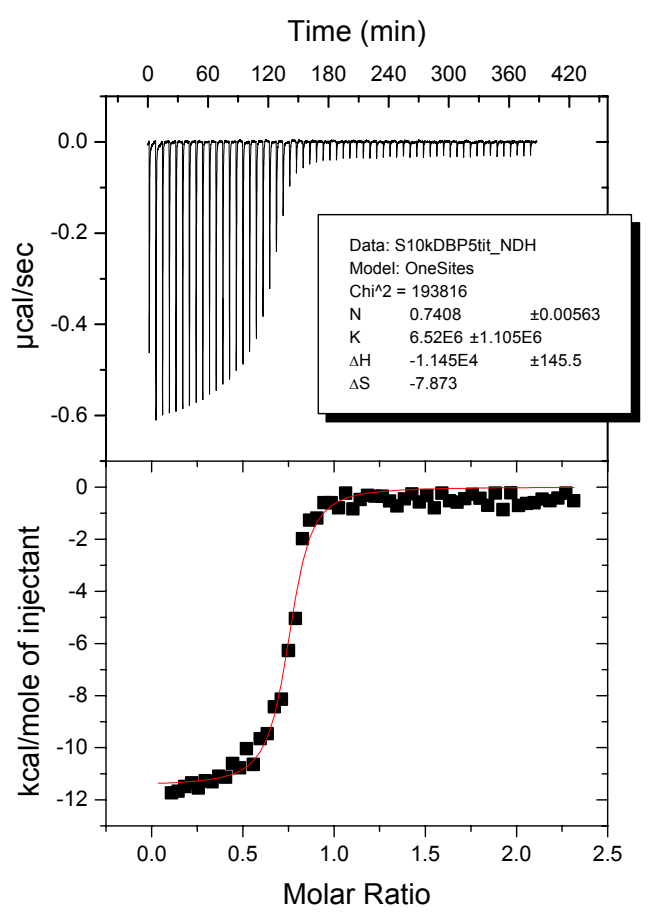

NBP-5(1-94) titrated with IGF 


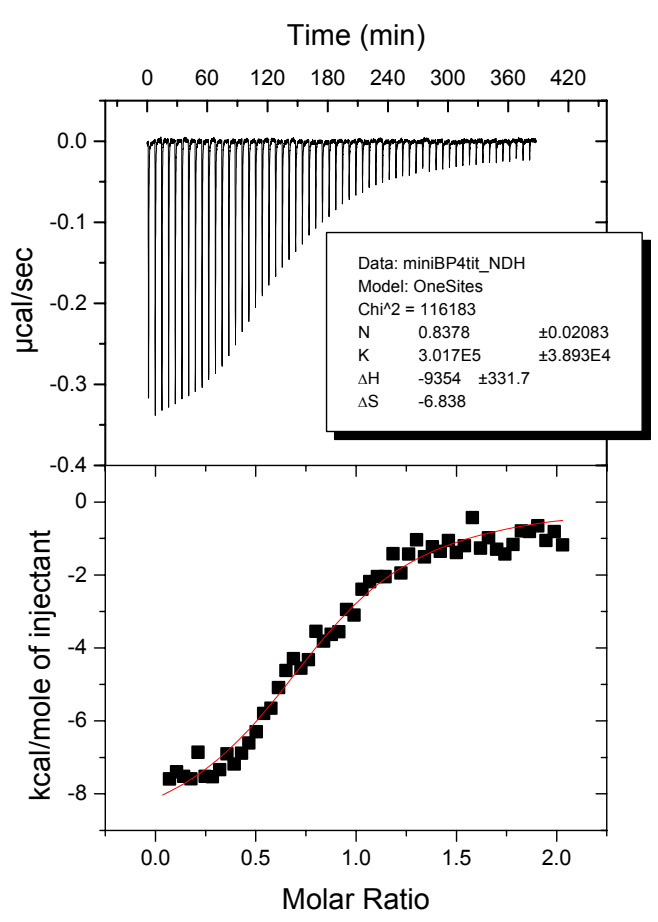

miniNBP-4 titrated with IGF

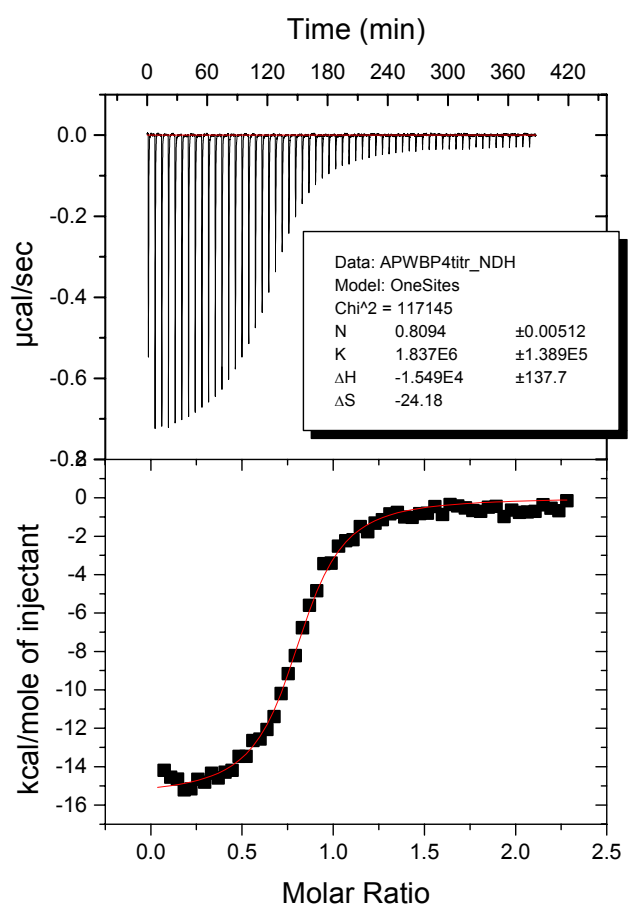

BP1-NBP-4 titrated with IGF

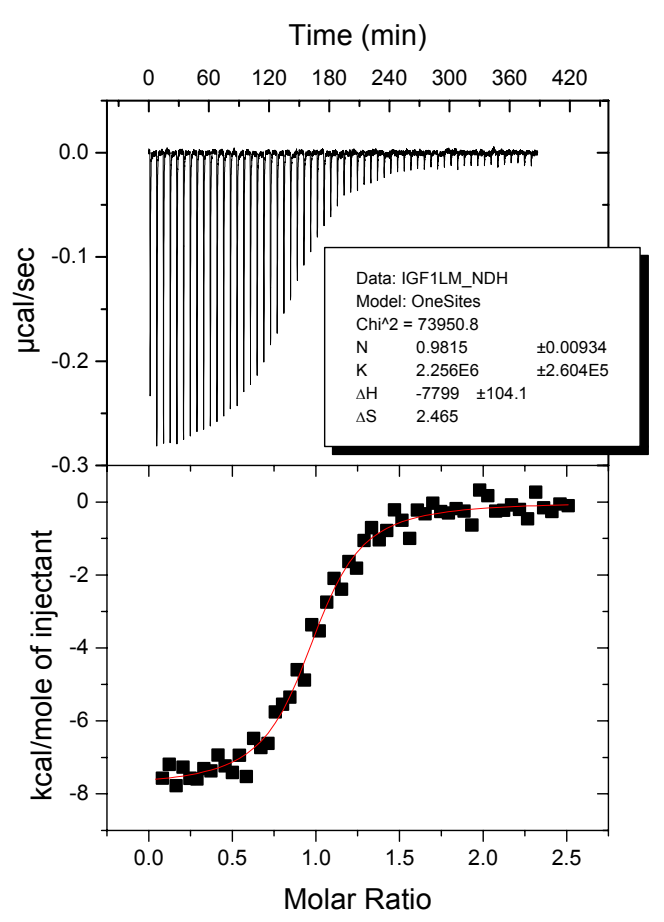

miniNBP5(L74M) titrated with IGF

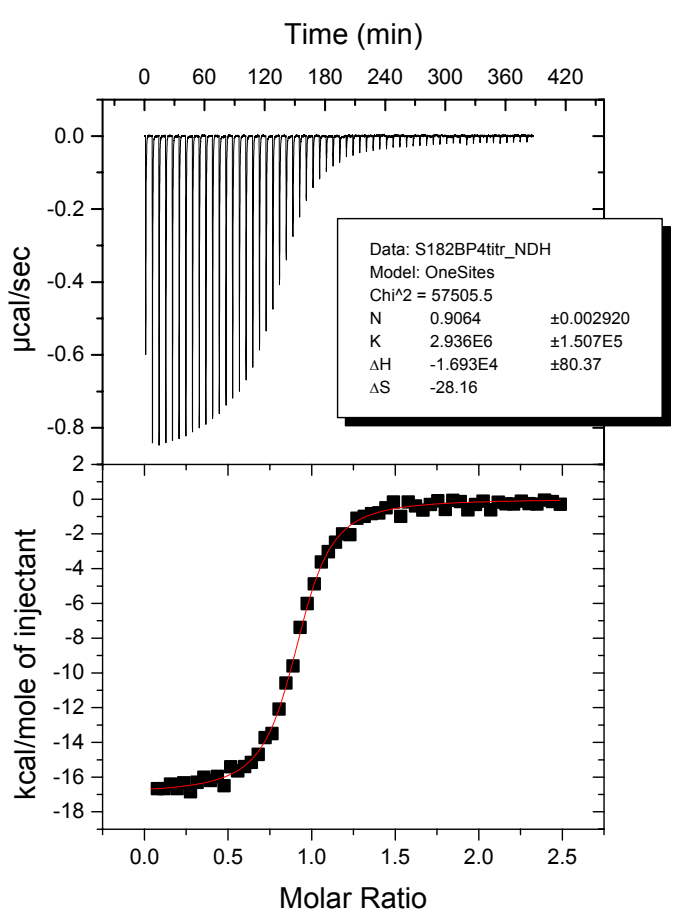

NBP-4(1-82) titrated with IGF 


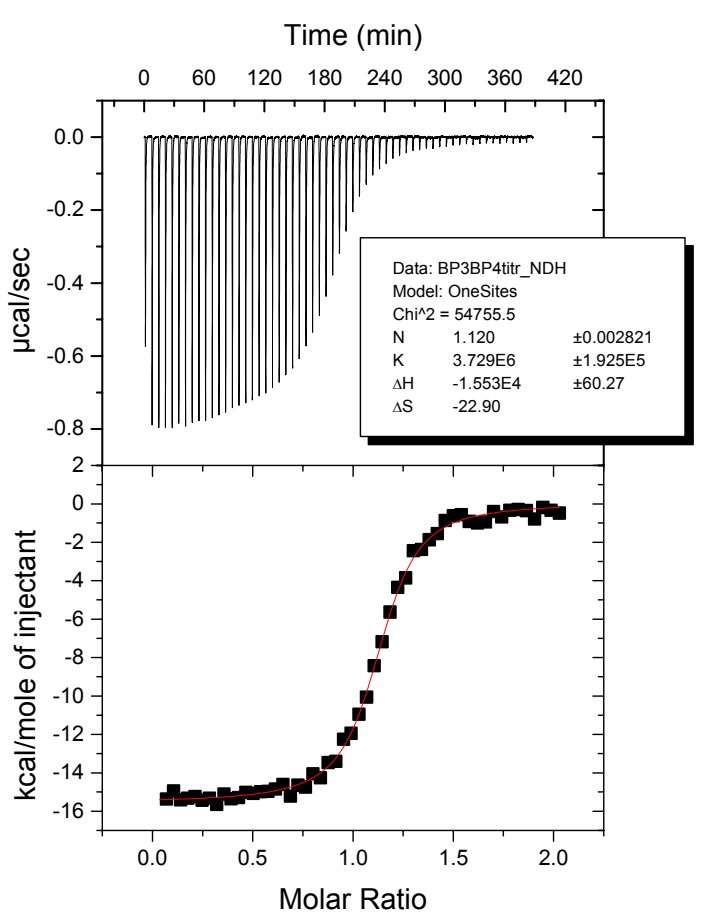

BP3-NBP-4 titrated with IGF

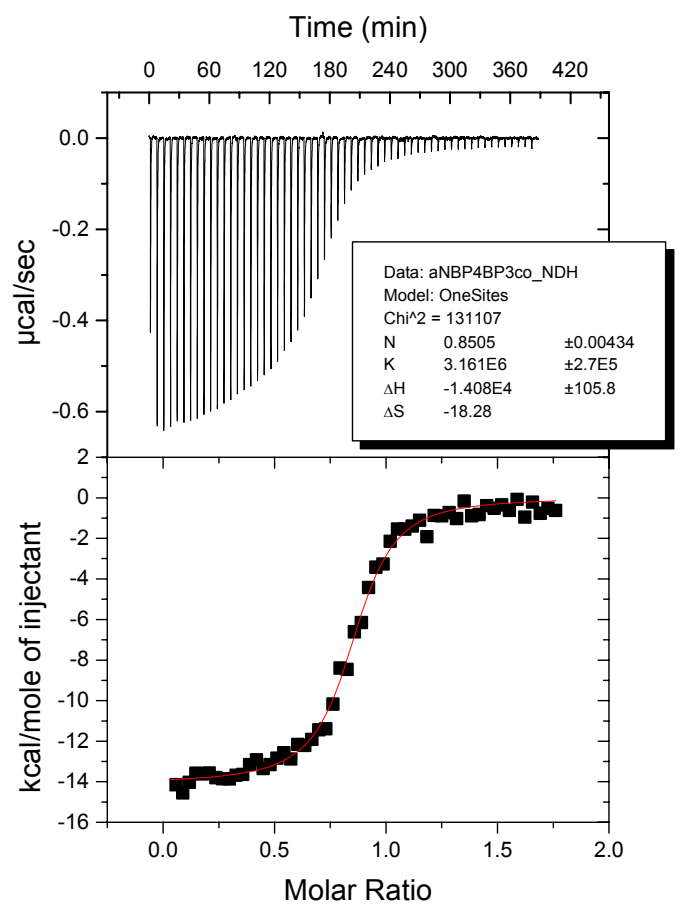

consBP3-NBP-4 titrated with IGF

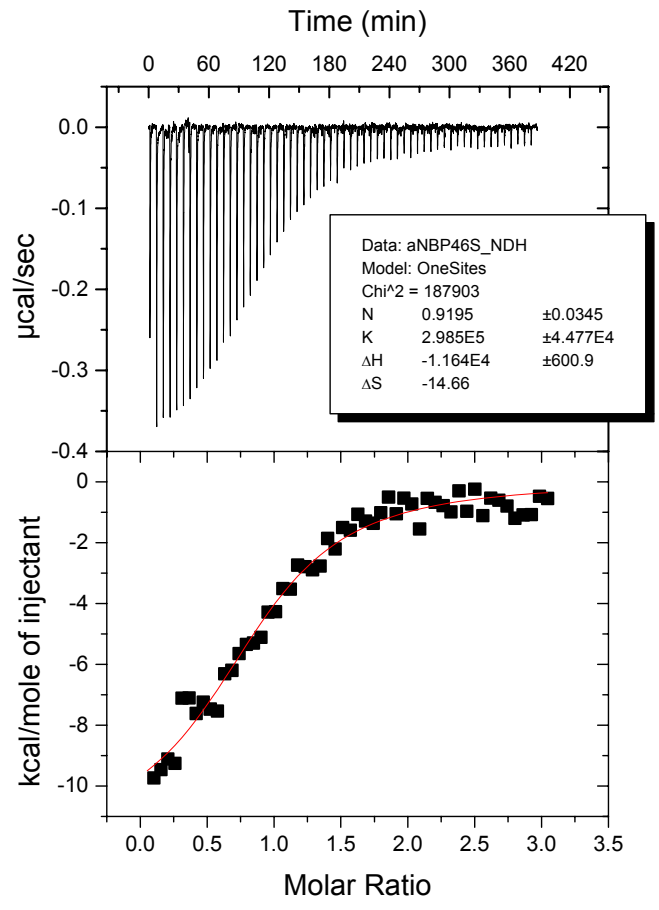

NBP4(G5-82) titrated with IGF

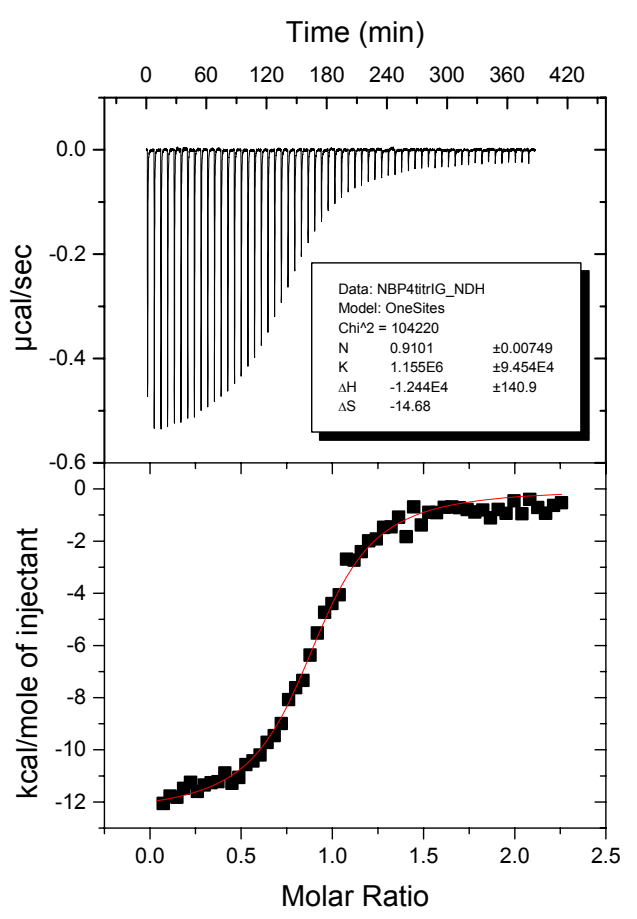

NBP-4(3-82) titrated with IGF 


\subsection{IGF-IR autophosphorylation inhibition assay}

+ Observed values

- Predicted values

$\mathrm{X}$ Protein concentration [nM]

$Y \%$ of inhibition
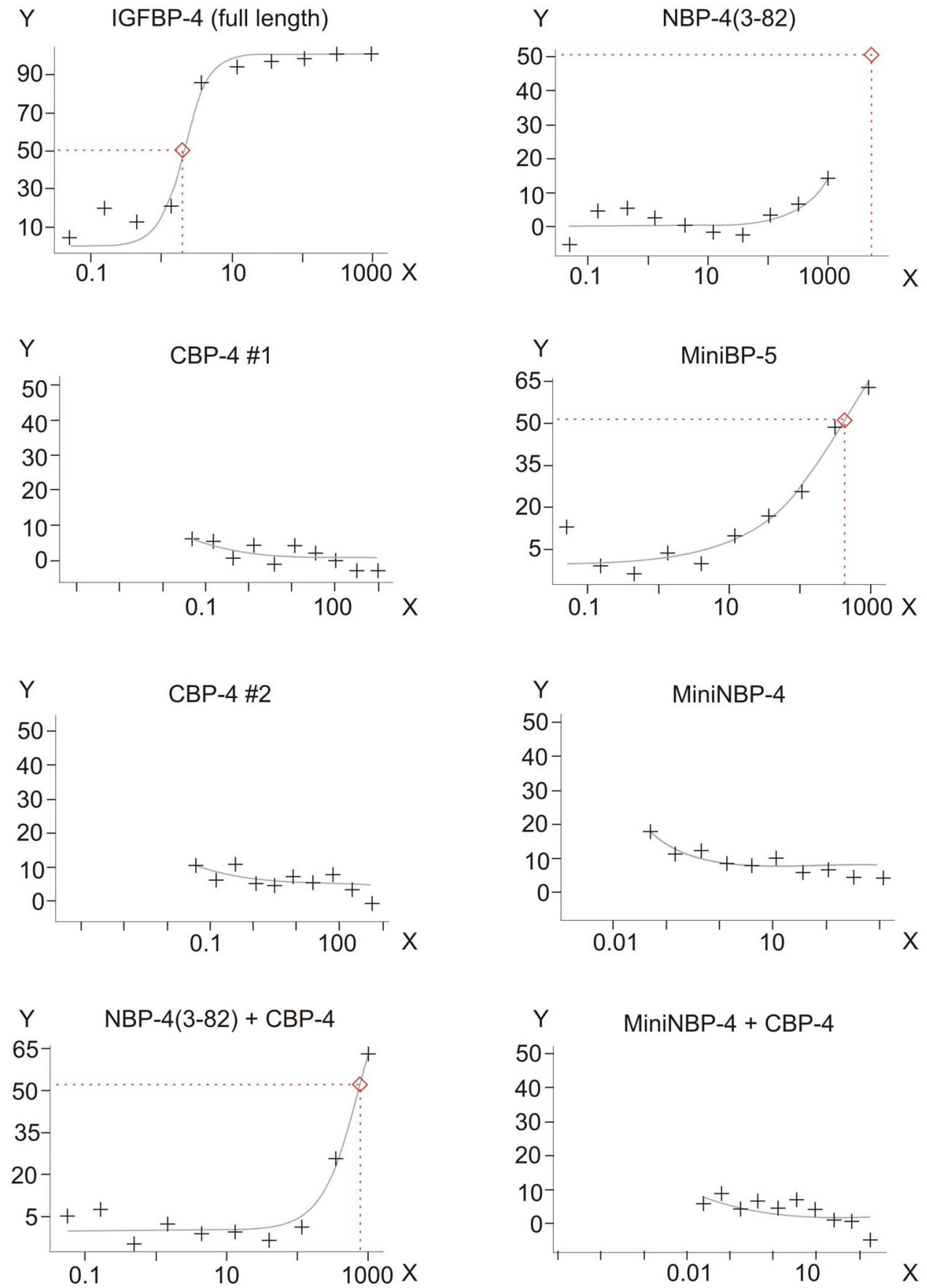


\section{References}

Adams, M.J., Blundell, T.L., Dodson, E.J., Dodson, G.G., Vijayan, M., Baker, E.N., Harding, M.M., Hodgkin, D.C., Rimmer, B. and Sheat, S. (1969) Structure of rhombohedral 2 zinc insulin crystals. Nature, 224, 491.

Adams, T.E., Epa, V.C., and Garrett, T.P.J. (2000) Structure of the type I insulin-like growth factor receptor. Cell. Mol. Life Sci. 57, 1050-93.

Ali, O., Cohen, P., and Lee, K.W. (2003) Epidemiology and biology of insulin-like growth factor binding protein-3 (IGFBP-3) as an anti-cancer molecule. Hor. Met. Res. 35, 726-733.

Andress, D.L., and Birnbaum, R.S. (1992) Human osteoblast-derived insulin-like growth factor (IGF) binding protein-5 stimulates osteoblast mitogenesis and potentiates IGF action. J. Biol Chem. 267, 22467-72.

Andress, D.L. (1995) Heparin modulates the binding of insulin-like growth factor (IGF) binding protein-5 to a membrane protein in osteoblastic cells. J. Biol. Chem. 47, 28289-28296.

Andress, D.L., Loop, S.M., Zapf, J., and Kiefer, M.C. (1993) Carboxy-truncated insulin-like growth factor binding protein-5 stimulates mitogenesis in osteoblast-like cells. Biochem. Biophys. Res. Commun. 195, 25-30.

Arteaga, C.L, Kitten, L.J., Coronado, E.B., Jacobs, S., Kull Jr., F.C., and Osborne, K. (1989) Blockade of the type I somatomedin receptor inhibits growth of human breast cancer cells in athymic mice. J. Clin. Invest. 84, 1418-1423.

August, G.P., Nissley, S.P., Kasuga, M., Lee, L., Greenstein, L., and Rechler, M.M. (1983) Purification of an insulin-like growth factor II receptor from rat chondrosarcoma cells. J. Biol. Chem. 258, 9033-6.

Babajko, S., and Binoux, M. (1996) Modulation by retinoic acid of insulin-like growth factor (IGF) and IGF binding protein expression in human SK-N-SH neuroblastoma cells. E. J. Endocrinology, 134, 474-480.

Bach, L.A., (1999) The insulin-like growth factor system: basic and clinical aspects. Aust. N.Z. J. Med. 29, 355-361.

Bach, L.A., and Rechler, M.M. (1995) Insulin-like growth factor binding proteins. Diabetes Rev. 3, 38-61.

Bach, L.A., Hsieh, S., Sakano, K., Fujiwara, H., Perdue, J.F., and Rechler, M.M. (1993) Binding of mutants of human insulin-like growth factor II to insulin-like growth factor binding proteins 1-6. J. Biol. Chem. 268, 9246-9254.

Ballard, F.J., Wallace, J.C., Francis, G.L., Read, L.C., and Tomas, F.M. (1996) Des (1-3)-IGF-I: a truncated form of insulin-like growth factor-I. Int. J. Biochem. Cell. Biol. 28, 1085-7.

Baserga, R. (1995) The insulin-like growth factor I receptor: a key to tumor growth? Cancer Res. 55, 249-52. 
Baxter, R.C. (1988) Characterisation of the acid-labile subunit of the growth hormonedependent insulin-like growth factor binding protein complex. J. Clin. Endocrinol. Metab. 67, 265-72.

Baxter, R.C. (1991) Insulin-like growth factor (IGF) binding proteins: the role of serum IGFBPs in regulating IGF availability. Acta Paediatr. Scand. 372, 107-14.

Baxter, R.C. (1994) Insulin-like growth factor binding proteins in the human circulation: a review. Horm. Res. 42, 140-4.

Baxter, R.C. (2000). Insulin-like growth factor (IGF)-binding proteins: interactions with IGFs and intrinsic bioactivities. Am. J. Physiol. 278, E967-E976.

Baxter, R.C., Bayne, M.L., and Cascieri, M. A. (1992) Structural determinants for binary and ternary complex formation between insulin-like growth factor-I (IGF-I) and IGF binding protein3. J. Biol. Chem. 267, 60-65.

Baxter, R.C., Martin, J.L., Tyler, M.I., and Howden, M.E. (1986) Growth-hormone-dependent insulin-like growth factor (IGF) binding protein from human plasma differs from other human IGF binding proteins. Biochem. Biophys. Res. Commun. 139, 1256-61.

Bayes-Genis, A., Schwartz, R.S., Lewis, D.A., Overgaard, M.T., Christiansen, M., Oxvig, C., Ashai, K., Holmes, D.R., and Conover, C.A. (2001) Insulin-like growth factor binding protein-4 protease produced by smooth muscle cells increases in the coronary artery after angioplasty. Arteriosclerosis, Thrombosis, and Vascular Biology, 21, 335-341.

Bayne, M.L., Applebaum, J., Chicchi, G.G., Miller, R.E., and Cascieri, M.A. (1990) The roles of tyrosine 24, 31, and 60 in the high affinity binding of insulin-like growth factor-I and the type 1 insulin-like growth factor receptor. J. Biol. Chem. 265, 15648-15652.

Beck, K.D., Knusel, B., and Hefti, F. (1993) The nature of the trophic action of brain- derived neurotrophic factor des(1-3)-insulin-like growth factor-1, and basic fibroblast growth factor on mesencephalic dopaminergic neurons developing in culture. Neurosci. 52, 855-66.

Binoux, M., lalou, C., and Mohseni-Zadeh, S. (1999) Biological actions of proteolytic fragments of the IGF binding proteins. In The IGF System: Molecular Biology, Physiology and Clinical Applications. M. Ron G. Rosenfeld, and J. Charles T. Roberts, Phd (eds.) Humana Press, Totowa, New Jersey. 281-313.

Blundell, T. L., Bedarkar, S., Rinderknecht, E. and Humbel, R. E. (1978) Insulin-like growth factor: a model for tertiary structure accounting for immunoreactivity and receptor binding, Proc. Natl Acad. Sci. USA, 75, 180-184.

Bohannon, N.J., Corp, E.S., Wilcox, B.J., Figlewicz, D.P., Dorsa, D.M., and Baskin, D.G. (1988) Localisation of binding sites for insulin-like growth factor-I (IGF-I) in the rat brain by quantitative autoradiography. Brain Res. 444, 205-13.

Boisclair, Y.R., Rhoads, R.P., Ueki, I. Wang, J. and. Ooi, G.T. (2001) Regulation of the acidlabile subunit of the $150-k D a$ IGF-binding protein complex and its role in the circulating IGF system. J. Anim. Sci. 79, E41-E47.

Boldt, H.B., Overgaard, M.T., Laursen, L.S., Weyer, K., Sottrup-Jensen, L., and Oxvig, C. (2001) Mutational analysis of the proteolytic domain of pregnancy-associated plasma protein-A (PAPP-A): classification as a metzincin. Biochemical Journal, 358, 359-367. 
Booth, B.A., Boes, M., Andress, D.L., Dake, B.L., Kiefer, M.C., Maack, C., Linhardt, R.J., Bar, K., Caldwell, E.E., Weiler, J. et al. (1995) IGFBP-3 and IGFBP-5 association with endothelial cells: role of C-terminal heparin binding domain. Growth Regulation, 5, 1-17.

Bramani, S., Song, H., Beattie, J., Tonner, E., Flint, D.J., and Allan, G.J. (1999) Amino acids within the extracellular matrix (ECM) binding region (201-218) of rat insulin-like growth factor binding protein (IGFBP)-5 are important determinants in binding IGF-I. J. Mol. Endocrinol. 23, 117-123.

Branden, C. and Tooze, J. (1999) Introduction to Protein Structure. Garland Publ., New York.

Braulke, T. (1999) Type-2 IGF receptor: a multi-ligand binding protein. Hormone and Metabolism Research, 31, 242-246.

Brünger, A.T., Adams, P.D., Clore, M.G., DeLano, W.L., Gros, P., Grosse-Kunstleve, R.W., Jiang, J., Kuszewski, J., Nilges, M., Pannu, N.S., Read, R.J., Rice, L.M., Simonson, T., and Warren, G.L. (1998) Crystallography and NMR System - A new software suite for macromolecular structure determination. Acta Crystallogr. D 54, 905-921.

Brzozowski, A.M., Dodson, E.J., Dodson, G.G., Murshudov, G.N., Verma, C., Turkenburg, J.P., de Bree, F.M., and Dauter, Z. (2002) Structural origins of the functional divergence of human insulin-like growth factor-I and insulin. Biochemistry 41, 9389-939.

Buckbinder, L., Talbott, R., Velasco-Miguel, S., Takenaka, I., Faha, B., Seizinger, B.R., and Kley, N. (1995) Induction of the growth inhibitor IGF-binding protein 3 by p53. Nature, 377, 646-649.

Buckway, C.K., Wilson, E.M., Ahlsen, M., Bang, P., Oh, Y., and Rosenfeld, R.G. (2001) Mutation of three critical amino acids of the N-terminal domain of IGF-binding protein-3 essential for high affinity IGF binding. J. Clin. Endoc. \& Metabol. 86. 4943-4950.

Bunn, R.C., and Fowlkes, J.L. (2003) Insulin-like growth factor binding protein proteolysis. Trends Endoc. \& Metabol. 14, 176-181.

Burgess, S.K., Jacobs, S., Cuatrecasas, P., and Sahyoun, N. (1987) Characterisation of a neuronal subtype of insulin-like growth factor-I receptor. J. Biol. Chem. 262, 1618-22.

Burstein, E.A., Vedenkina, N.S., and Ivkova, M.N. (1973) Fluorescence and the location of tryptophan residues in protein molecules. Photochem. Photobiol. 18, 263-279.

Burtrum, D., Zhu, Z., Lu, D., Anderson, D.M., Prewett, M., Pereira, D.S., Bassi, R., Abdullah, R., Hooper, A.T., Koo, H., Jimenez, X., Johnson, D., Apblett, R., Kussie, P., Bohlen, P., Witte, L., Hicklin, D.J., and Ludwig, D.L. (2003) A fully human monoclonal antibody to the insulin-like growth factor I receptor blocks ligand-dependent signaling and inhibits human tumor growth in vivo. Cancer Research, 63, 8912-8921.

Butt, A.J., Firth, S.M., King, M.A., and Baxter, R.C. (2000) Insulin-like growth factor-binding protein-3 modulates expression of Bax and $\mathrm{Bcl}-2$ and potentiates p53-independent radiationinduced apoptosis in human breast cancer cells. J. Biol. Chem. 275, 39174-39181.

Byun, D., Mohan, S., Baylink, D.J., and Qin, X. (2001a) Localization of the IGF binding domain and evaluation of the role of cysteine residues in IGF binding in IGF binding protein-4. Journal of Endocrinology, 169, 135-143. 
Byun, D., Mohan, S., Yoo, M., Sexton, C., Baylink, D.J., and Qin, X. (2001b) Pregnancyassociated plasma protein-A accounts for the insulin-like growth factor (IGF)-binding protein-4 (IGFBP-4) proteolytic activity in human pregnancy serum and enhances the mitogenic activity of IGF by degrading IGFBP-4 in vitro. Journal of Clinical Endocrinology and Metabolism, 86, 847-854.

Carlsson-Skwirut, C., Jornvall, H., Holmgren, A., Andersson, C., Bergman, T., Lundquist, B., and Sara, V.R. (1986) Isolation and characterisation of variant IGF-I as well as IGF-2 from adult human brain. FEBS Lett. 201, 46-50.

Carlson-Skwirut, C., Lake, M., Hartmanis, M., Hall, K., and Sara, V.R. (1989) A comparison of the biological activity of the recombinant intact, and truncated insulin-like growth factor 1 (IGF1). Biochim. Biophys. Acta. 1011, 192-7.

Carrick, F.E., Forbes, B.E., and Wallace, J.C. (2001) BIAcore analysis of bovine insulin-like growth factor (IGF)-binding protein-2 identifies major IGF binding site determinants in both the amino- and carboxyl-terminal domains. J. Biol. Chem. 276, 27120-27128.

Carrick, F.E., Wallace, J.C., and Forbes, B.E. (2002) The interaction of Insulin-like growth factors (IGFs) with Insulin-like growth factor binding proteins (IGFBPs): a review. Lett. Pep. Sci. 8, 147-153.

Cascieri, M.A., Chicchi, G.C., Applebaum, J., Hazes, N.S., Green, B.C., and Bayne, M.L. (1988). Mutants of human insulin-like growth factor I with reduced affinity for the type I insulinlike growth factor receptor. Biochemistry, 27, 3229-3233.

CCP4, Collaborative Computational Project, Number 4. (1994) The CCP4 Suite: Programs for protein crystallography. Acta Cryst., D50, 760-763.

Chan, K., and Spencer, E.M. (1998) Megakaryocytes endocytose insulin-like growth factor (IGF) I and IGF-binding protein-3: a novel mechanism directing them into alpha granules of platelets. Endocrinology, 139, 559-65.

Chan, J.M., Stampfer, M.J., Giovannucci, E., Gann, P.H., Ma, J., Wilkinson, P., Henneken, C.H., and Pollak, M. (1998) Plasma insulin-like growth factor-I and prostate cancer risk: A prospective study. Science, $279,563-566$.

Chelius, D., Baldwin, M.A., Lu, X., and Spencer, E.M. (2001) Expression, purification and characterization of the structure and disulfide linkages of insulin-like growth factor binding protein-4. J. Endocrinol. 168, 283-96.

Chen, C., Zhu, Y.F., Liu, X.J., Lu, Z.X., Xie, Q., and Ling, N. (2001) Discovery of a series of nonpeptide small molecules that inhibit the binding of insulin-like growth factor (IGF) to IGFbinding proteins. J Med Chem. 44,:4001-10.

Chernausek, S.D., Smith, C.E., Duffin, K.L., Busby, W.H., Wright, G., and Clemmons, D.R (1995) Proteolytic cleavage of insulin-like growth factor binding protein 4 (IGFBP-4) Localization of cleavage site to non-homologous region of native IGFBP-4. J. Biol. Chem. 270, 11377-11382.

Cheung, P.T., Wu, J., Banach, W., and Chernausek, S.D. (1994) Glucocorticoid regulation of an insulin-like growth factor-binding protein-4 protease produced by a rat neuronal cell line. Endocrinology, 135, 1328-35. 
Chorney, M.J., Chorney, K., Seese, N., Owen, M.J., and Daniels, J.(1998) A quantitative trait locus associated with cognitive ability in children. Psych. Sci. 9, 159-166.

Chung, C.T. Niemela, S.L., Miller, R.H. (1989) One-step preparation of competent Escherichia coli: Transformation and storage of bacterial cells in the same solution PNAS, 86, 2172-2175.

Clemmons, D.R., Cascieri, M.A., Camacho-Hübner, C., Mc-Cusker, R.H., and Bayne, M.L. (1990) Discrete alterations of the insulin-like growth factor I molecule which alter its affinity for insulin-like growth factor-binding proteins result in changes in bioactivity. J. Biol. Chem. 265, $12210-12216$.

Clemmons, D.R. (1999) IGF binding proteins and extracellular matrix. In Rosenfeld, R.G., and Roberts, C.T. (eds), The IGF system. Molecular biology, physiology, and clinical applications. Humana Press, Totowa, pp. 273-279.

Clemmons, D.R. (2001) Use of mutagenesis to probe IGF-binding protein structure/function relationships. Endocr. Rev. 22, 800-817.

Cohen, P., Graves, H.C., Peehl, D.M., Kamarei, M., Guidice, L.C., and Rosenfeld, R.G. (1992) Prostate-specific antigen (PSA) is an insulin-like growth factor binding protein-3 protease found in seminal plasma. J. Clin. Endocrinol. Metab. 75, 1046-53.

Cohen, P., Lamson, G., Okajima, T., and Rosenfeld, R.G. (1993) Transfection of the human insulin-like growth factor binding protein-3 gene into Balb/c fibroblasts inhibits cellular growth. Mol. Endocrinol. 7, 380-386.

Cohen, P., Peehl, D.M., Graves, H.C., and Rosenfeld, R.G. (1994) Biological effects of prostate specific antigen as an insulin-like growth factor binding protein-3 protease. J. Endocrinol. 142, 407-15.

Cohen, P., Clemmons, D.R., and Rosenfeld, R.G. (2000) Does the GH-IGF axis play a role in cancer pathogenesis? Growth Hor. IGF Res. 10, 297-305.

Cole, M.D. (1986) The myc oncogene: its role in transformation and differentiation. Annu. Rev. Genet. 20, 361-84.

Conover, C.A., Oxvig, C., Overgaard, M.T., Christiansen, M., and Giudice, L.C. (1999) Evidence that the insulin-like growth factor binding protein-4 protease in human ovarian follicular fluid is pregnancy associated plasma protein-A. Journal of Clinical Endocrinology and Metabolism, 84, 4742-4745.

Conover, C.A., Faessen, G.F., Ilg, K.E., Chandrasekher, Y.A., Christiansen, M., Overgaard, M.T., Oxvig, C., and Giudice, L.C. (2001) Pregnancy-associated plasma protein-A is the insulin-like growth factor binding protein-4 protease secreted by human ovarian granulosa cells and is a marker of dominant follicle selection and the corpus luteum. Endocrinology, 142, 2155.

Cooke, R.M., Harvey, T.S., and Campbell, I.D. (1991) Solution structure of human insulin-like growth factor 1: a nuclear magnetic resonance and restrained molecular dynamics study. Biochemistry, 30, 5484-5491.

Datta, S.R., Dudeck, H., Tao, X., Masters, S., Fu, H., Gotoh, Y., and Greenberg, M.E. (1997) Akt phosphorylation of BAD couples' survival signals to intrinsic machinery. Cell, 91, 231-41. 
Daughaday, W.H., Hall, K., Raben, M.S., Salmon Jr., W.D., Van den Brande, J.L., and Van Wyk, J.J. (1972) Somatomedin: proposed designation for sulphation factor. Nature, 235, 107.

Daughaday, W., and Salmon Jr, W.D. (1999) Historical perspective: the origins and development of the somatomedin hypothesis. In The IGF system: Molecular bilogy, physiology and clinical applications. Rosenfeld, R.G., and Roberts, C.T. (eds),. Humana Press, Totowa, New Jersey. 1-15.

Delbé, J., Blat, C., Desauty, G., and Harel, L. (1991) Presence of IDF45 (mIGFBP-3) binding sites on chick emryo fibroblasts. Biochem Biophys. Res. Commun. 179, 495-501.

De Meyts, P., Wallach, B., Christoffersen, C.T., Urso, B., Gronskov, K., Latus, L.J., Yakushiji, F., Ilondo, M.M., and Shymko, R.M. (1994) The insulin-like growth factor-I receptor: Structure, ligand-binding mechanism and signal transduction. Horm. Res. 42, 152-69.

Drenth, J. (1994) Principles of protein X-ray crystallography. Springer-Verlag, New York

Du, K., and Montminy, M. (1998) CREB is a regulatory target for the protein kinase Akt/PKB. J. Biol. Chem. 273, 32377-9.

Dubaquie, Y. and Lowman, H.B. (1999) Total alanine-scanning mutagenesis of insulin-like growth factor I (IGF-I) identifies differential binding epitopes for IGFBP-1 and IGFBP-3. Biochemistry, 38, 6386-96.

Fanayan, S., Firth, S.M., Butt, A.J., and Baxter, R.C. (2000) Growth inhibition by insulin-like growth factor binding protein-3 in T47D breast cancer cells requires transforming growth factor- $\beta$ (TGF- $\beta$ ) and type II TGF- $\beta$ receptor. J. Biol. Chem. 275, 39146-39151.

Firth, S.M., and Baxter, R.C. (2002) Cellular actions of the insulin-like growth factor binding proteins. Endocr. Rev. 23, 824-854

Firth, S.M., Clemmons, D.R., and Baxter, R.C. (2001) Mutagenesis of basic amino acids in the carboxyl-terminal region of insulin-like growth factor binding protein-5 affects acid-labile subunit binding. Endocrinology, 14, 2147-2150.

Firth, S.M., Ganeshprasad, U., and Baxter, R.C. (1998) Structural determinants of ligand and cell surface binding of insulin-like growth factor-binding protein-3. J. Biol. Chem. 273, 26312638.

Forbes, B., Szabo, L., Baxter, R.C., Ballard, F.J., and Wallace, J.C. (1988) Classification of the insulin-like growth factor binding proteins into three distinct categories according to their binding specificities. Biochem. Biophys. Res. Commun. 157, 196-202.

Forbes, B.E., Turner, D., Hodge, S.J., McNeil, K.A., Forsberg, G., and Wallace, J.C. (1998) Localization of an insulin-like growth factor (IGF) binding site of bovine IGF binding protein-2 using disulfide mapping and deletion mutation analysis of the C-terminal domain. J. Biol. Chem. 273, 4647-4652.

La Fortelle, E. de and Bricogne, G. (1997) Maximum-likelihood heavy-atom parameter refinement in MIR and MAD methods. Meth. Enzym. 276, 472-494.

Fowlkes, J., and Freemark, M. (1992) Evidence for a novel insulin-like growth factor (IGF)dependent protease regulating IGF-binding protein-4 in dermal fibroblasts. Endocrinology, 131, 2071-2076. 
Francis, G.L., Upton, F.M., Ballard, F.J., McNeil, K.A., and Wallace, J.C. (1988) Insulin-like growth factors 1 and 2 in bovine colostrum. Sequences and biological activities compared with those of a potent truncated form. Biochemical J. 251, 95-103.

Frost, R.A., and Tseng L. (1991) Insulin-like growth factor binding protein-1 is phosphorylated by cultured human endometrial stromal cells and multiple protein kinases in vitro. J. Biol. Chem. 266, 18082-18088.

Furstenberger, G., and Senn, H.J. (2002) Insulin-like growth factors and cancer. Lancet Oncol. $3,298$.

Galanis, M., Firth, S.M., Bond, J., Nathanielsz, A., Kortt, A.A., Hudson, P.J., and Baxter, R.C. (2001) Ligand- binding characteristics of recombinant amino- and carboxyl-terminal fragments of human insulin-like growth factor-binding protein-3. J. Endocrinol. 169, 123-133.

Gallicchio, M.A., Kneen, M., Hall, C., Scott, A.M., and Bach, L.A. (2001) Overexpression of insulin-like growth factor binding protein-6 inhibits rhabdomyosarcoma growth in vivo. Int. J. Cancer. 94, 645-651.

Garrett, T.P., McKern, N.M., Lou, M., Frenkel, M.J., Bentley, J.D., Lovrecz, G.O., Elleman, T.C., Cosgrove, L.J., and Ward, C.W. (1998) Crystal structure of the first three domains of the type-1 insulin-like growth factor receptor. Nature, 394, 395-9.

Giacovazzo, C., Monaco, H.L., Viterbo, D., Scordari, F., Gilli, G., Zanotti, M. and Catti, M. (1992) Fundamentals of crystallography. Oxford University Press, Oxford.

Gill, S.C., and von Hippel, P.H. (1989) Calculation of protein extinction coefficients from amino acid sequence data. Anal. Biochem. 182, 319-26.

Girnita, A., Girnita, L., del Prete, F., Bartolazzi, A., Larsson, O., and Axelson, M. (2004) Cyclolignans as inhibitors of the insulin-like growth factor-1 receptor and malignant cell growth. Cancer Research, 64, 236-42.

Grellier, P., Degalle, B., Babajko, S. (1998) Expression of insulin-like growth factor-binding protein 6 complementary DNA alters neuroblastoma cell growth. Cancer Res. 58, 1670-1676.

Grzmil, M., Hemmerlein, B., Thelen, P., Schweyer, S., and Burfeind, P. (2004) Blockade of the type I IGF receptor expression in human prostate cancer cells inhibits proliferation and invasion, up-regulates IGF binding protein-3, and suppresses MMP-2 expression. J. Pathol. 202, 50-9.

Giudice, L.C., Conover, C.A., Bale, L., Faessen, G.H., Ilg, K., Sun, I., Imani, B., Suen, L.-F., Irwin, J.C., Christiansen, M., Overgaard, M.T., and Oxvig, C. (2002) Identification and Regulation of the IGFBP-4 Protease and Its Physiological Inhibitor in Human Trophoblasts and Endometrial Stroma: Evidence for Paracrine Regulation of IGF-II Bioavailability in the Placental Bed during Human Implantation. The Journal of Clinical Endocrinology \& Metabolism, 87, 2359-66.

Halloran, B.P., and Spencer, E.M. (1988) Dietary phosphorus and 1,25-dihydroxyvitamin D metabolism: influence of insulin-like growth factor I. Endocrinology, 123, 1225-9.

Hashimoto, R., Ono, M., Fujiwara, H., Higashihashi, N., Yoshida, M., Enjohkimura, T., and Sakano, K. (1997) Binding sites and binding properties of binary and ternary complexes of insulin-like growth factor-II (IGF-II), IGF-binding protein-3, and acid-labile subunit. J. Biol. Chem. 272, 27936-42. 
Hassan, A.B. (2003) Keys to the hidden treasures of the mannose 6-phosphate/insulin-like growth factor 2 receptor. American Journal of Pathology, 162, 3-6.

Headey, S.J., Keizer, D.W., Yao, S., Wallace, J.C., Bach, L.A., and Norton, R.S. (2004a) Binding site for the C-domain of insulin-like growth factor (IGF) binding protein-6 on IGF-II; implications for inhibition of IGF actions. FEBS Lett. 568, 19-22.

Headey, S.J., Keizer, D.W., Yao, S., Brasier, G., Kantharidid P., Bach, L.A., and Norton, R.S. (2004b) C-terminal domain of insulin-like growth factor binding protein-6: structure and interaction with insulin-like growth factor-II Mol. Endocrinol. 18, 2740-50.

Headey, S.J., Leeding, K.S., Norton, R.S., and Bach, L.A. (2004c) Contributions of the N- and C-terminal domains of IGF binding protein-6 to IGF binding. J. Mol. Endocr. 33, 377-86.

Heidenreich, K.A., Freidenberg, G.R., Figlewicz, D.P., and Gilmore, P.R. (1986) Evidence for a subtype of insulin-like growth factor I receptor in brain. Regul. Pept. 15, 301-10.

Hernandez-Sanchez, C., Blakesley, V., Kalebic, T., Helman, L., and LeRoith, D. (1995) The role of the tyrosine kinase domain of the insulin-like growth factor-I receptor in intracellular signalling, cellular proliferation and tumorgenesis. J. Biol. Chem. 270, 29176-81.

Ho, P.J., and Baxter, R.C. (1997) Characterization of truncated insulin-like growth factorbinding protein-2 in human milk. Endocrinology, 138, 3811-3818.

Hodgson, D.R., May, F.E.B., and Westley, B.R. (1996) Involvement of phenylalanine 23 in the binding of IGF-1 to the insulin and type I IGF receptor. Regul. Pept. 66, 191-196.

Hoeck, W.G., and Mukku, V.R. (1994) Identification of the major sites of phosphorylation in IGF binding protein-3. J. Cell. Biochem. 56, 262-273.

Hoppe, W. (1957) Die Faltmolekülmethode: eine neue Methode zur Bestimmung der Kristallstruktur bei ganz oder teilweise bekannten Molekülstrukturen. Acta Cryst. 10, 750-1.

Huang, S.S., Leal, S.M., Chen, C-L., Liu, I-H., and Huang, J.S. (2004) Identification of insulin receptor substrate proteins as key molecules for the T $\beta R$-V/LRP-1-mediated growth inhibitory signaling cascade in epithelial and myeloid cells. FASEB, 18, 1230-54.

Huber, R. (1965) Die automatisiere Faltmolekülmethode. Acta Cryst. 19, 353-6.

Humbel, R.E. (1990) Insulin-like growth factor-I, and II. Eur. J. Biochem. 190, 445-62.

Hwa, V., Oh, Y., Burren, C.P., Choi, W.K., Graham, D.L., Ingermann, A., Kim, H.S., LopezBermejo, A., Minniti, G., Nagalla, S.R., Pai, K., Spagnoli, A., Vorwerk, P., Wanek, D.L.V., Wilson, E.M., Yamanaka, Y., Yang, D.H., and Rosenfeld, R.G. (1999) The IGF binding protein superfamily. In Rosenfeld, R.G., and Roberts, C.T. (eds), The IGF system. Molecular biology, physiology, and clinical applications. Humana Press, Totowa, pp. 315-327.

Imai, Y., Moralez, A., Andag, U., Clarke, J.B., Busby, W.H., and Clemmons, D.R. (2000) Substitutions for hydrophobic amino acids in the N-terminal domains of IGFBP-3 and-5 markedly reduce IGF-I binding and alter their biologic actions. J. Biol. Chem. 275, 18188-94.

Jones, J.I., D'Ercole, A.J., Camacho-Hubner, C., and Clemmons, D.R. (1991) Phosphorylation of insulin-like growth factor (IGF)-binding protein 1 in cell culture and in vivo: effects on affinity for IGF-I. Proc. Natl. Acad. Sci. U S A, 88, 7481-5. 
Jones, J.L., Gockerman, A., Busby, W.H., Wrigh, G. and Clemmons, D.R. (1993) Insulin-like growth factor binding protein 1 stimulates cell migration and binds to the a5b integrin receptor by means of its Arg-Gly-Asp sequence. Proc. Natl. Acad. Sci. USA, 90, 10553-7.

Jones, J.L., and Clemmons, D.R. (1995) Insulin-like growth factors and their binding proteins: biological actions. Endocr. Rev. 16, 3-34.

Jung, G.W., Spencer, E.M., and Lue, T.F. (1998) Growth hormone enhances regeneration of nitric oxide synthase-containing penile nerves after cavernous nerve neurotomy in rats. Journal of Urology, 160, 1899-904

Kabsch, W. (1993) Automatic processing of rotation diffraction data from crystals of initially unknown symmetry and cell constants. J. Appl. Cryst. 26, 795-800.

Kalus, W., Zweckstetter, M., Renner, C., Sanchez, Y., Georgescu, J., Grol, M., Demuth, D., Schumacher, R., Dony, C., Lang, K., and Holak, T.A. (1998) Structure of the IGF binding domain of the insulin-like growth factor-binding protein-5 (IGFBP-5): implications for IGF and IGF-I receptor interactions. EMBO J. 17, 6558-72.

Kanzaki, S., Hilliker, S., Baylink, D.J., and Mohan, S. (1994) Evidence that human bone cells in culture produce insulin-like growth factor-binding protein- 4 and -5 proteases. Endocrinology, 134, 383-92.

Kelley, K.M., Oh, Y., Gargosky, S.E., Gucev, Z., Matsumoto, T., Hwa, V., Ng, L., Simpson, D.M., and Rosenfeld, R.G. (1996) Insulin-like growth factor-binding proteins (IGFBPs) and their regulatory dynamics. Int. J. Biochem. and Cell Biol. 28, 619-637.

Khandwala, H.M., McCutcheon, I.E., Flyvbjerg, A., and Friend, K.E. (2000) The effects of insulin-like growth factors on tumorigenesis and neoplastic growth. Endocr. Rev. 21, 215-44.

Kim, B., Cheng, H.L., Margolis, B., and Feldman, E.L. (1998) Insulin receptor substrate 2 and Shc play different roles in insulin-like growth factor-I signalling. J. Biol. Chem. 273, 34543-50.

Kim, B., Leventhal, P.S., Saltiel, A.R., and Feldman, E.L. (1997) Insulin-like growth factor-I mediated neurite outgrowth in vitro requires mitogen-activated protein kinase activation. J. Biol. Chem. 272, 21268-73.

Knudtson, K.L., Boes, M., Sandra, A., Dake, B.L., Booth, B.A., and Bar, R.S. (2001) Distribution of chimeric IGF binding protein (IGFBP)-3 and IGFBP-4 in the rat heart: importance of C-terminal basic region. Endocrinology, 142, 3749-3755.

Kornfeld, S. (1992) Structure and function of the mannose-6-phosphate/Insulin-like growth factor II receptors. Annu. Rev. Biochem. 61, 307-30.

Ladokhin, A.S. (2000) Fluorescence Spectroscopy in Peptide and Protein Analysis. in Encyclopedia of Analytical Chemistry, R.A. Meyers (Ed.), pp. 5762-79.

Lakowicz, J. 1999. Principles of Fluorescence Spectroscopy. Plenum, New York.

Lalou, C., Lassarre, C., and Binoux, M. (1996). A proteolytic fragment of insulin-like growth factor (IGF) binding protein-3 that fails to bind IGFs inhibits the mitogenic effects of IGF-I and insulin. Endocrinology, 137, 3206-12. 
Lamzin, V.S., and Wilson, K.S. (1993) Automated refinement of protein models. Acta Cryst. D49, $129-47$.

Landale, E.C., Strong, D.D., Mohan, S. and Baylink, D.J. (1995) Sequence comparison and predicted structure for the four exon-encoded regions of human insulin-like growth factor binding protein 4. Growth Factors, 12, 245-50.

Lassarre, C., and Binoux, M. (1994) Insulin-like growth factor binding protein-3 is functionally altered in pregnancy plasma. Endocrinology, 134, 1254-62.

Lawrence, J.B., Bale, L.K., Haddad, T.C., Clarkson, J.T., and Conover, C.A. (1999a) Characterization and partial purification of the insulin-like growth factor (IGF)-dependent IGF binding protein-4-specific protease from human fibroblast conditioned media. Growth Hormone and IGF Research, 9, 25-34.

Lawrence, J.B., Oxvig, C., Overgaard, M.T., Sottrup-Jensen, L., Gleich, G.J., Hays, L.G., Yates, J.R., and Conover, C.A. (1999b) The insulin-like growth factor (IGF)-dependent IGF binding protein-4 protease secreted by human fibroblasts is pregnancy-associated plasma protein-A. PNAS, 96, 3149-53.

Leal, S.M., Huang, S.S., and Huang, J.S. (1999) Interactions of high affinity insulin-like growth factor-binding proteins with the type $\mathrm{V}$ transforming growth factor- $\beta$ receptor in mink lung epithelial cells. J. Biol. Chem. 274, 6711-7.

Leal, S.M., Liu, Q., Huang, S.S., and Huang, J.S. (1997) The type V transforming growth factor $\beta$ receptor is the putative insulin-like growth factor binding protein 3 receptor. J. Biol. Chem. 272, 20572-6.

Lee, K-W., Liu, B., Ma, L., Li, H., Bang, P., Koeffler H.P., and Cohen, P. (2004) Cellular Internalization of insulin-like growth factor binding protein-3. J. Biol. Chem. 279, 469-76.

LeRoith, D. and Helman, L. (2004) The new kid on the block(ade) of the IGF-1 receptor. Cancer Cell, 5, 201-2.

LeRoith, D., Werner, H., Faria, T.N., Kato, H., Adamo, M., and Roberts, C.T. (1993) Insulin-like growth factor receptor: implications for nervous function. Ann. NY Acad. Sci. 692, 22-32.

LeRoith, D., Werner, H., Beitner-Johnson, D., and Roberts, C.T. (1995) Molecular and cellular aspects of the insulin-like growth factor I receptor. Endocr. Rev. 16, 143-63.

Lesniak, M.A., Hill, J.M., Kiess, W., Rojeski, M., Pert, C.B., and Roth, J. (1988) Receptors for insulin-like growth factors I and II: Autoradiographic localisation in rat brain and comparison to receptors for insulin. Endocrinology, 123, 2089-99.

Leventhal, P.S., Russel, J.W., and Feldman, E.L. (1999) IGFs and the nervous system. In The IGF System. R.G. Rosenfeld and C. Roberts (eds). Humana Press, Totowa, New Jersey.

Li, W., Fawcett, J., Widmer, H.R., Fielder, P.J., Rabkin, R., and Keller, G.A. (1997) Nuclear transport of insulin-like growt factor-I and insulin-like growth factor binding protein-3 in opossum kidney cells. Endocrinology, 138, 1763-6.

Lin, T.M., Galbert, S.P., Kiefer, D., Spellacy, W.N., and Gall, S. (1974) Characterization of four human pregnancy-associated plasma proteins. American Journal of Obstetrics and Gynecology, 118, 223-36. 
Liu, X.J., Xie, Q., Zhu, Y.F., Chen, C., Ling, N. (2001) Identification of a nonpeptide ligand that releases bioactive insulin-like growth factor-I from its binding protein complex. J. Biol. Chem. $31,32419-22$.

Loddick, S.A., Liu, X.J., Lu, Z.X., Liu, C.L., Behan, D.P., Chalmers, D.C., Foster, A.C., Vale, W.W., Ling, N., and Desouza, E.B. (1998) Displacement of insulin-like growth factors from their binding proteins as a potential treatment for stroke. Proc. Nat. Acad. Sci. USA, 95, 1894-8.

MacDonald, R.G., Pfeffer, S.R., Coussens, L., Tepper, M.A., Brocklebank, C.M., Mole, J.E., Anderson, J.K., Chen, E., Czech, M.P., and Ulrich, A. (1988) A single receptor binds both insulin-like growth factor II and mannose-6-phosphate. Science, 329, 1134-37.

Maile, L.A., Gill, Z.P., Perks, C.M., and Holly, J.M. (1999) The role of cell surface attachment and proteolysis in the insulin-like growth factor (IGF)-independent effects of IGF-binding protein-3 on apoptosis in breast epithelial cells. Endocrinology, 140, 4045-5.

Mannhardt, B., Weinzimer, S.A., Wagner, M., Fiedler, M., Cohen, P., Jansen-Durr, P., and Zwerschke, W. (2000) Human papillomavirus type 16 E7 oncoprotein binds and inactivates growth-inhibitory insulin-like growth factor binding protein 3. J. Mol. Cell. Biol. 20, 6483-95.

Martin, J. L., Ballesteros, M., and Baxter, R. C. (1992) Insulin-like growth factor-I (IGF-I) and transforming growth factor-beta 1 release IGF-binding protein-3 from human fibroblasts by different mechanisms. Endocrinology 131, 1703-10.

Martin, J.L, and Baxter, R.C. (1999) IGF binding proteins as modulators of IGF action. In The IGF system: Molecular bilogy, physiology and clinical applications. M. Ron G. Rosenfeld, and J. Charles T. Roberts, Phd (eds) Humana Press, Totowa, New Jersey. 227-55.

Martin, J.L., Coverley, J.A., Pattison, S.T., and Baxter, R.C. (1995) IGFBP-3 and IGFBP-6 production by MCF-7 breast cancer cells: Stimulation by retinoic acid and cyclic AMP, and differential effects of estradiol. Endocrinology 136, 1219-1226.

Makrides, S.C. (1996) Strategies for achieving high-level expression of genes in Escherichia coli. Microbiol. Rev., 60, 512-538.

Mann, K.G., and Lungblad, R.L. (1982) Biochemistry of thrombin. In: Colman, R.W., Hirsh, J., Marder, V.J., Salzman, E.W. (eds) Hemostasis and Thrombosis. Lippincott, Philadelphia, pp 112-126.

Mazerbourg, S., Callebaut, I., Zapf, J., Mohan, S., Overgaard, M., and Monget, P. (2004) Update on IGFBP-4: regulation of IGFBP-4 levels and functions, in vitro and in vivo, Growth Hormone \& IGF Research, 14, 71-84.

McCusker, R.H., Camacho-Hubner, C., Bayne, M.L., Cascieri, M.A., and Clemmons, D.R. (1990) Insulin-like growth factor (IGF) binding to human fibroblast and glioblastoma cells: the modulating effect of cell released IGF binding proteins (IGFBPs). J Cell Physiol. 144, 244-253.

McCusker, R.H., and Clemmons, D.R. (1997) Use of lanthanum to accurately quantify insulinlike growth factor binding to proteins on cell surfaces. J. Cell. Biochem. 66, 256-267.

McCusker, R.H., Kaleko, M., Sackett, R.L. (1998) Multivalent cations and ligand affinity of the type 1 insulin-like growth factor receptor on P2A2-LISN muscle cells. J. Cell. Physiol. 176, 392-401.

McPherson, A. (1999) Crystallization of Biological Macromolecules. CSHL Press, New York 
McRee, D.E. (1999) XtalView/Xfit - A versatile program for manipulating atomic coordinates and electron density. J. Struc. Biol. 125, 156-65.

Mishra, S., and Murphy, L. J. (2003) Phosphorylation of insulin-like growth factor (IGF) binding protein-3 by breast cancer cell membranes enhances IGF-I binding. Endocrinology, 144, 4042-50.

Monget, P., Mazerbourg, S., Delpuech, T., Maurel, M.C., Maniere, S., Zapf, J., Lalmanach, G., Oxvig, C., and Overgaard, M.T. (2003) Pregnancy-associated plasma protein-A is involved in insulin-like growth factor binding protein-2 (IGFBP-2) proteolytic degradation in bovine and porcine preovulatory follicles: identification of cleavage site and characterization of IGFBP-2 degradation. Biology of Reproduction, 68, 77-86.

Morgan, D.O., Edman, J.C., Standring, D.N., Fried, V.A., Smith, M.C., Roth, R.A., and Rutter, W.J. (1987) Insulinlike growth factor II receptor as a multifunctional binding protein. Nature, $329,301-7$.

Myers, M.G., Grammer, T.C., Wang, L.M., Sun, X.J., Pierre, J.H., Blenis, J., and White, M.F. (1994) Insulin receptor substrate -1 mediates phosphatidylinositol- 3'- kinase and p70 signalling during insulin, IGF-1 and interleukin-4 stimulation. J. Biol. Chem. 269, 28783-9.

Myers, M.G. Jr, and White, M.F. (1996) Insulin signal transduction and the IRS proteins. Annu. Rev. Pharmacol. Toxicol. 36, 615-58.

Nam, T.J., Busby, W.H., and Clemmons, D.R. (1994) Human fibroblasts secrete a serine protease that cleaves insulin-like growth factor binding-5. Endocrinology, 135, 1385-1391.

Nam, T., Moralez, A., and Clemmons, D. (2002) Vitronectin Binding to IGF Binding Protein-5 (IGFBP-5) Alters IGFBP-5 Modulation of IGF-I Actions. Endocrinology, 143, 30-36

Nemato, S. and Finkel, T. (2004) Ageing and the mystery at Arles. Nature 429, 149-152.

Neumann, G.M., and Bach, L.A. (1999) The N-terminal disulfide linkages of human insulin-like growth factor-binding protein-6 (hIGFBP-6) and hIGFBP-1 are different as determined by mass spectrometry. J. Biol. Chem. 274, 14587-14594.

Neuenschwander, S., Roberts Jr, C.T., and LeRoith, D. (1995) Growth inhibition of MCF-7 breast cancer cells by stable expression of an insulin-like growth factor I receptor antisense ribonucleic acid. Endocrinology, 136, 4298-4303.

Nissley, S.P., and Kiess, W. (1991) Binding of insulin-like growth factor II (IGF-II) and lysosomal enzymes to the IGF-II/mannose-6-phosphate receptor. Modern concepts of insulinlike growth factors. EM Spencer, ed. Elsevier Science Publishing Co., Inc. New York, 419-30.

Oh, Y., Müller, H.L., Lamson, G., and Rosenfeld, R. (1993) Insulin-like growth factor (IGF)independent action of IGF-binding protein-3 in Hs578T human breast cancer cells. Cell surface binding and growth inhibition. J. Biol. Chem. 268, 14964-71.

Oh, Y., Müller, H.L., Ng, L., and Rosenfeld, R. (1993) Transforming growth factor-beta-induced cell growth inhibition in human breast cancer cells is mediated through insulin-like growth factor binding protein-3 action. J. Biol. Chem. 270,13589-92. 
Payet, L.D., Wang, X.H., Baxter, R.C., and Firth, M. (2003) Amino- and carboxyl-terminal fragments of insulin-like growth factor (IGF) binding protein-3 cooperate to bind IGFs with high affinity and inhibit IGF receptor interactions. Endocrinology, 144, 2797-806.

Perdue, J.F., Bach L.A., Hashimoto, R., Sakano, K., Fujita-Yamaguchi, Y., Fujiwara, H., and Rechler, M.M. (1994) in: The insulin-like growth factors and their regulatory proteins, Baxter, R.C., Gluckman, P.D., and Rosenfeld, R.G. (eds), Elsevier Science Publishing Co., New York, pp. 67-76.

Perks, C.M., Newcomb, P.V., Norman, M.R., and Holly, J.M. (1999) Effect of insulin-like growth factor binding protein-1 on integrin signaling and the induction of apoptosis in human breast cancer cells. J. Mol. Endocrinol. 22, 141-50.

Perrakis, A., Morris, R., and Lamzin, V.S. (1999) Automated protein model building combined with iterative structure refinement. Nature Struct. Biol. 6, 458-63.

Perrakis, A., Harkiolaki, M., Wilson, K.S., and Lamzin, V.S. (2001) ARP/wARP and molecular replacement. Acta Cryst. D57, 1445-50.

Pollak, M.N., Schernhammer, E.S., and Hankinson, S.E. (2004) Insulin-like growth factors and neoplasia. Nature Rev. Cancer, 4, 505-18.

Prior, T.I., Helman, L.J., FitzGerald, D.J., and Pastan, I. (1991) Cytotoxic activity of a recombinant fusion protein between insulin-like growth factor I and Pseudomonas exotoxin. Cancer Research, 51, 174-80.

Rehm, T., Huber, R., and Holak, T.A. (2002) Application of NMR in structural proteomics: screening for proteins amenable to structural analysis. Structure, 10, 1613-18.

Qin, X., Byun, D., Strong, D.D., Baylink, D.J. and Mohan, S. (1999) Studies on the role of human insulin-like growth factor-II (IGF-II)-dependent IGF binding protein (hIGFBP)-4 protease in human osteoblasts using protease-resistant IGFBP-4 analogs. Journal of Bone and Mineral Research, 14, 2079-2088.

Qin, X.Z., Strong, D.D., Baylink, D.J., and Mohan, S. (1998) Structure-function analysis of the human insulin-like growth factor binding protein-4. J. Biol. Chem. 273, 23509-16.

Rajah, R., Lee, K.W., and Cohen, P. (2002) Insulin-like growth factor binding protein-3 mediates tumor necrosis factor-alpha-induced apoptosis: role of $\mathrm{Bcl}-2$ phosphorylation. Cell Growth Differ. 13, 163-71.

Rajah, R., Valentinis, B., and Cohen, P. (1997) Insulin-like growth factor (IGF)-binding protein3 induces apoptosis and mediates the effects of transforming growth factor-b1 on programmed cell death through a p53- and IGF-independent mechanism. J. Biol. Chem. 272, 12181-8.

Rechler, M.M. (1985) The nature and regulation of the receptors for insulin-like growth factors. Ann. Rev. Physiol. 47, 425-42.

Rechler, M.M. (1993) Insulin-like growth factor-binding proteins. Vitam. Horm. 47, 1-114.

Rechler, M.M. (1997) Growth inhibition by insulin-like growth factor (IGF) binding protein-3What's IGF got to do with it? Endocrinolgy, 138, 2645-7. 
Rinderknecht, E. and, Humbel, R.E. (1976) Amino-terminal sequences of two polypeptides from human serum with nonsuppressible insulin-like and cell-growth promoting activities: evidence for structural homology with insulin B chain. Proc. Natl. Acad. Sci. USA, 73, 4379-81.

Rinderknecht, E., and, Humbel, R.E. (1978a) The amino acid sequences of human insulin-like growth factor-I and its structural homology with proinsulin. J. Biol. Chem. 253, 2769-76.

Rinderknecht, E., and Humbel, R.E. (1978b) Primary structure of human insulin-like growth factor II. FEBS Lett. 89, 283-6.

Rosenberg, A.H., Goldman, E., Dunn, J.J., Studier, F.W. and Zubay, G. (1993) Effects of consecutive AGG codons on translation in Escherichia coli, demonstrated with a versatile codon test system. J. Bacteriol. 175, 716-22.

Rozhkova, A., Stirnimann, C.U., Frei, P., Grauschopf, U., Brunisholz, R., Grutter, M.G., Capitani, G., and Glockshuber, R. (2004) Structural basis and kinetics of inter- and intramolecular disulfide exchange in the redox catalyst DsbD. EMBO J. 23, 1709-19.

Russo, V.C., Bach, L.A., Fosang, A.J., Baker, N.L., and Werther, G.A. (1997) Insulin-like growth factor binding protein-2 binds to cell surface proteoglycans in the rat brain olfactory bulb. Endocrinology, 138, 4858-67.

Sackett, R.L., McCusker, R.H., (1998a) Multivalent cations depress ligand binding to cellassociated insulin-like growth factor binding protein-5 on human glioblastoma cells. Endocrinology, 139, 1943-51.

Sackett, R.L., McCusker, R.H., (1998b) Multivalent cations depress ligand affinity of insulin-like growth factor-binding proteins-3 and -5 on human GM-10 fibroblast cell surfaces. J. Cell. Biochem. 69, 364-75.

Sakano, K., Enjoh, T., Numata, F., Fujiwara, H., Marumoto, Y., Higashihashi, N., Sato, Y., Perdue, J.F., and Fujita-Yamaguchi Y. (1991) The design, expression, and characterization of human insulin-like growth factor II (IGF-II) mutants specific for either the IGF-II/cationindependent mannose 6-phosphate receptor or IGF-I receptor J. Biol. Chem. 266, 31, 2062635.

Sara, V.R., Carlsson-Skwirut, C., Anderson, C., Hall, E., Sjorgen, B., Holmgren, A, and Jornvall, H. (1986) Characterisation of somatomedins from human foetal brain: identification of a variant form of insulin-like growth factor I. Proc. Natl. Acad. Sci. USA, 83, 4904-7.

Sato, H., Nishimura, S., Ohkubo, T., Kyoguku, Y., Koyama, S. and Kobayashi, Y. (1993) Three-dimensional structure of human insulin-like growth factor I (TGF-I) determined by ${ }^{1} \mathrm{H}$ NMR and distance geometry, Int. J. Pept. Protein Res. 41, 433-40.

Schagger, H., von Jagow, G. (1987) Tricine-sodium dodecyl sulfate-polyacrylamide gel electrophoresis for the separation of proteins in the range from 1 to $100 \mathrm{kDa}$. Anal Biochem. 166, 368-79.

Scharf, J.G., and Braulke, T. (2003) The role of the IGF axis in hepatocarcinogenesis. Horm. Metab. Res. 35, 685-93.

Schedlich, L.J., Young, T., Firth S., and Baxter, R. (1998) Insulin-like growth factor-binding protein (IGFBP9-3 and IGFBP-5 share a common nuclear trasport pathway in T47D human breast carcinoma cells. J. Biol. Chem. 273, 18347-52. 
Schedlich, L.J., O'Han, M.K., Leong, G.M., and Baxter, R.C. (2004) Insulin-like growth factor binding protein-3 prevents retinoid receptor heterodimerization: implications for retinoic acidsensitivity in human breast cancer cells. Bioch. Bioph. Res. Comm. 314, 83-8.

Schlechter, N.L., Russel, S.M., Spencer, E.M., and Nicoll, C.S. (1986) Evidence suggesting that the direct growth-promoting effect of growth hormone on cartiliage in vivo is mediated by local production of somatomedin. Proc. Natl. Acad. Sci. 83, 7932-4.

Schneider, M.R., Wolf, E., Hoeflich, A., and Lahm H. (2002) IGF-binding protein-5: flexible player in the IGF system and effector on its own. Journal of Endocrinology, 172, 423-40.

Shand, J.H., Beattie, J., Song, H., Phillips, K., Kelly, S.M., Flint, D.J., and Allan, G.J. (2003) Specific amino acid substitutions determine the differential contribution of the $\mathrm{N}$ - and $\mathrm{C}$ terminal domains of insulin-like growth factor (IGF)-binding protein-5 in binding IGF-I. J. Biol. Chem. 278, 17859-66.

Singh, B., Charkowicz, D., and Mascarenhas, D. (2004) Insulin-like growth factor-independent effects mediated by a C-terminal metal-binding domain of Insulin-like growth factor binding protein-3. J. Biol. Chem. 279, 477-87.

Ständker, L., Braulke, T., Mark, S., Mostafavi, H., Meyer, M., Honing, S., Gimenez-Gallego, G., and Forssmann, W.G. (2000) Partial IGF affinity of circulating N- and C-terminal fragments of human insulin-like growth factor binding protein-4 (IGFBP-4) and the disulfide bonding pattern of the C-terminal IGFBP-4 domain. Biochemistry, 39, 5082-8.

Stoll, R., Renner, C., Hansen, S., Palme, S., Klein, C., Belling, A., Zeslawski, W., Kamionka, M., Rehm, T., Mühlhahn, P., Schumacher, R., Hesse, F., Kaluza, B., Voelter, W., Engh, R.A., and Holak, T.A. (2001) Chalcone derivatives antagonize interactions between the human oncoprotein MDM2 and p53. Biochemistry, 40, 336-44.

Suh, D.Y., Hunt, T.K., and Spencer E.M. (1992) Insulin-like growth factor-I reverses the impairment of wound healing induced by corticosteroids in rats. Endocrinology, 131, 2399-406.

Suikkari, A-M., and Baxter, R.C. (1992) Insulin-like growth factor binding protein-3 is functionally normal in pregnancy serum. J. Clin. Endocrinol. Metab. 74, 177-83.

Takahashi, H., Nakanishi, T., Kami, K., Arata, Y., Shimada, I. (2000) A novel NMR method for determining the interfaces of large protein-protein complexes. Nature Struct. Biol. 7, 7220-223.

Taylor, V.L., and Spencer, E.M. (2001) Characterisation of insulin-like growth factor-binding protein-3 binding to a novel receptor on human platelet membranes. J. Endocrinol. 168, 30715.

Thrailkill, K.M., Quarles, L.D., Nagase, H., Suzuki, K., Serra, D.M., and Fowlkes, J.I. (1995) Characterization of insulin-like growth factor-binding protein-5-degrading proteases produced throughout murine osteoblast differentiation. Endocrinology, 136, 3527-33.

Torres, A.M., Forbes, B.E., Aplin, S.E., Wallace, J.C., Francis, G.L., and Norton, R.S. (1995) Solution structure of human insulin-like growth factor II. Relationship to receptor and binding protein interactions. J. Mol. Biol. 248, 385-401.

Turner, R., and Tjian, R. (1989) Leucine repeats and an adjacent DNA binding domain mediate the formation of functional cFos-cJun heterodimers. Science, 243, 1689-94. 
Twigg, S.M., Kiefer, M.C., Zapf, J., and Baxter, R.C. (2000) A central domain binding site in insulin-like growth factor binding protein-5 for the acid-labile subunit. Endocrinology, 141, 4547.

Vajdos, F.F., Ultsch, M., Schaffer, M.L., Deshayes, K.D., Liu, J., Skelton, N.J., and de Vos, A.M. (2001) Crystal structure of human insulin-like growth factor-1: detergent binding inhibits binding protein interactions. Biochemistry, 40, 11022-29.

Valentinis, B., Bhala, A., DeAngelis, T., Baserga, R., and Cohen, P. (1995) The human insulinlike growth factor (IGF) binding protein-3 inhibits the growth of .broblasts with a targeted disruption of the IGF-I receptor gene. Mol. Endocrinol. 9, 361-7.

Vivian, J.T., and Callis, P.R. (2001) Mechanisms of tryptophan fluorescence shifts in proteins. Biophysical Journal, 80, 2093-109.

Vorwerk, P., Hohmann, B., Oh, Y., Rosenfeld, R.G., and Shymko, R.M. (2002) Binding properties of insulin- like growth factor binding protein-3 (IGFBP-3), IGFBP-3 N- and C-terminal fragments, and structurally related proteins mac25 and connective tissue growth factor measured using a biosensor. Endocrinology, 143, 1677-85.

Wang, J-F., Hampton, B., Mehlman, T., Burgess, W.H., and Rechler, M.M. (1988) Isolation of a biologically active fragment from the carboxy-terminus of the fetal rat binding protein for insulin-like growth factors. Biochem. Biophys. Res. Commun. 157, 718-26.

Weber, T., Baumgartner, R., Renner, C., Marahiel, M.A., and Holak, T.A. (2000) Solution structure of PCP, a prototype for the peptidyl carrier domains of modular peptide synthetases. Struct. Fold. Des. 8, 407-18.

Wetterau, L.A., Moore, M.G., Lee, K.W., Shim, M.L., and Cohen, P. (1999) Novel aspects of the insulin-like growth factor binding proteins. Mol. Gen. Metab. 68, 161-81.

White, M.F. and Kahn, C.R. (1989) Cascade of autophosphorylation in the beta-subunit of the insulin receptor. J. Cell. Biochem. 39, 429-41.

Wu, H.B., Kumar, A., Tsai, W.C., Mascarenhas, D., Healey, J., and Rechler, M. M. (2000) Characterization of the inhibition of DNA synthesis in proliferating mink lung epithelial cells by insulin-like growth factor binding protein-3. J. Cell. Biochem. 77, 288-97.

Wu, Y., Cui, K., Miyoshi, K., Hennighausen, L., Green, J.E., Setser, J., LeRoith, D., and Yakar, S. (2003) Reduced circulating insulin-like growth factor I levels delay the onset of chemically and genetically induced mammary tumors. Cancer Res. 63, 4384-8.

Wüthrich, K. (1986) NMR of Proteins and Nucleic Acids, Wiley-Interscience, New York.

Yamasaki, H., Prager, D., and Melmed, S. (1993) Structure-function of the human insulin-like growth factor-I receptor: a discordance of somatotrophin internalisation and signalling. Mol. Endocrinol. 7, 681-5.

Yao, S.G., Headey, S.J., Keizer, D.W., Bach, L.A., and Norton, R.S. (2004. C-terminal domain of insulin-like growth factor (IGF) binding protein 6: Conformational exchange and its correlation with IGF-II binding. Biochemistry. 43, 11187-95.

Zeslawski, W., Beisel, H.G., Kamionka, M., Kalus, W., Engh, R.A., Huber, R., Lang, K., and Holak T.A. (2001) The interaction of insulin-like growth factor-I with the N-terminal domain of IGFBP-5. EMBO J. 20, 3638-44. 
Zhang, X. and Yee, D. (2002) Insulin-like growth factor binding protein-1 (IGFBP-1) inhibits breast cancer cell motility. Cancer research, 62, 4369-75.

Zheng, B., Clarke, J.B., Busby, W.H., Duan, C., and Clemmons, D.R. (1998) Insulin-like growth factor-binding protein- 5 is cleaved by physiological concentrations of thrombin. Endocrinology, $139,1708-14$.

Zhu YF, Wilcoxen K, Gross T, Connors P, Strack N, Gross R, Huang CQ, McCarthy JR, Xie Q, Ling N, Chen C. (2003) 6,7-dihydroxyisoquinoline-3-carboxylic acids are potent inhibitors on the binding of insulin-like growth factor (IGF) to IGF-binding proteins: optimization of the 1position benzoyl side chain. Bioorg. Med. Chem. Lett.,13,1927-30

Xu, Q., Yan, B., Li, S., and Duan, C. (2004) Fibronectin binds insulin-like growth factor-binding protein 5 and abolishes its ligand-dependent action on cell migration. J. Biol. Chem. 279, 4269-77. 